\title{
Zeitaufgelöste inelastische \\ Neutronenstreuung an entmischenden Silber-Natriumchlorid-Einkristallen
}

\author{
Dissertation zur Erlangung des Doktorgrades der \\ Mathematisch-Naturwissenschaftlichen Fakultäten \\ der Georg-August-Universität zu Göttingen
}

\author{
vorgelegt von \\ Dirk Caspary
}

aus Delmenhorst

Göttingen, 2002 
D7

Referent: Prof. Dr. G. Eckold

Korreferent: Prof. Dr. H. G. Wagner

Tag der mündlichen Prüfung: 31. Oktober 2002 


\section{Inhaltsverzeichnis}

Inhaltsverzeichnis 3

1 Einleitung 5

1.1 Entmischungsreaktionen . . . . . . . . . . . . . 7

1.2 Gitterdynamische Grundlagen . . . . . . . . . . . . . . . . . 13

1.3 Elastische Neutronenstreuung . . . . . . . . . . . . . 18

1.4 Inelastische Neutronenstreuung . . . . . . . . . . . . . . . 20

2 Das Silber-Natriumchlorid System 25

$\begin{array}{lll}3 & \text { Experimente } & \mathbf{3 7}\end{array}$

3.1 Probenpräparation . . . . . . . . . . . . . . . 37

3.2 Neutronenstreuexperimente . . . . . . . . . . . . . . . . . . 43

3.3 Probenöfen für die Neutronenstreuexperimente . . . . . . . . . . . . . 45

3.4 Die Zeitauflösung . . . . . . . . . . . . . . . . . . . . . . . . . . . . . 49

4 Ergebnisse der statischen Experimente $\quad \mathbf{5 5}$

4.1 Dispersionskurven im homogenen Zustand . . . . . . . . . . . . . . . 55

4.2 Das Frequenz-Konzentrations Diagramm . . . . . . . . . . . . 59

4.3 Braggreflexe nach langen Auslagerungszeiten . . . . . . . . . . . 63

4.4 Dispersionskurven nach langen Auslagerungszeiten . . . . . . . . . . . 67

5 Ergebnisse der kinetischen Experimente $\quad \mathbf{7 5}$

5.1 Kinetik im instabilen Bereich . . . . . . . . . . . . . . . . 75

5.2 Entmischung im natriumreichen Kristall . . . . . . . . . . . . . . . 84

5.3 Entmischung in den späten Stadien . . . . . . . . . . . . . . . . 90

$\begin{array}{lll}6 & \text { Zusammenfassung } & 91\end{array}$

$\begin{array}{ll}\text { Literaturverzeichnis } & 95\end{array}$

$\begin{array}{ll}\text { Abbildungsverzeichnis } & 103\end{array}$

$\begin{array}{ll}\text { Abbildungsanhang } & 105\end{array}$ 


\section{Einleitung}

Wenn im alltäglichen Leben kondensierte Materie beschrieben wird, werden erfahrbare Eigenschaften wie Dichte, Farbe, Formbeständigkeit oder Temperatur herangezogen. Diese Eigenschaften sind genauso ohne die Kenntnis über die atomistische Struktur bestimmbar. Doch alle Eigenschaften sind letztendlich auf die atomistische Struktur und die Wechselwirkung zwischen den Atomen und/oder Molekülen, den Teilchen, zurückzuführen. In Molekülen treten starke Wechselwirkungen zwischen den Atomen auf, die durch die chemische Bindung beschrieben werden. Soll die chemische Bindung untersucht werden, ist ein Experiment so zu wählen, daß die Moleküle sich nicht gegenseitig beeinflussen. Sie kann sehr gut in Gasphasenexperimenten mit kleinen Drücken untersucht werden, weil dann der Einfluß von Wechselwirkungen der Moleküle untereinander vernachlässigbar ist. Sind die Wechselwirkungen zwischen Teilchen jedoch groß genug, kondensieren sie, und bei starken Wechselwirkungen kommt es zu einer periodischen Anordnung der Teilchen: Es bilden sich Kristallstrukturen. Selbst wenn in einem Kristall die Teilchenzahl in der Größenordnung von $10^{23}$ Teilchen liegt, ist die Beschreibung des Kristalls durch seine periodische Natur relativ einfach möglich, da nur die kleinste Einheit der periodischen Struktur bekannt sein muß. Mit dieser Elementarzelle kann dann der gesamte Kristall beschrieben werden.

Mit der phänomenologischen Thermodynamik ist es möglich, Zustände und Eigenschaften von Materie zu beschreiben, ohne detailliert auf die atomistische Struktur einzugehen. Gleichwohl können die Änderungen bei einem Übergang von einem Zustand in einen anderen dargestellt werden. Sie macht aber keine Aussagen über die Natur, den Mechanismus und die damit verbundene Kinetik der Änderung. Eine Brücke vom diskreten Aufbau der Materie zu den makroskopischen Eigenschaften fester Körper bilden die Kristallographie, die sich mit der Anordnung der Atome in Kristallgittern beschäftigt, und die Gitterdynamik, die dynamischen Aspekte wie z.B. Gitterschwingungen oder Diffusion im Kristallgitter untersucht. Mit der Gitterdynamik ist es möglich, anhand von Modellen für die Teilchen-Teilchen Wechselwirkung auf mikroskopischer Ebene, makroskopische Eigenschaften wie thermische Ausdehnung, Wärmekapazität, die spektroskopische Natur, elastische Eigenschaften, Phasenumwandlungen u.v.m. aus mikroskopisch Eigenschaften abzuleiten. Dies führt zu einem tieferen Verständnis der Eigenschaften und der dynamischen Prozesse in der kondensierten Materie.

Ein gutes Beispiel für die Diskrepanz zwischen einer thermodynamischen Beschrei- 
bung und der atomistischen Struktur sind Entmischungsreaktionen: Es sei von einem System aus zwei Substanzen A und B ausgegangen, die oberhalb einer kritischen Temperatur $T_{\text {krit }}$ mischbar seien und unterhalb der kritischen Temperatur nicht. Wenn ein Gemisch von A und B von $T>T_{\text {krit }}$ auf $T<T_{\text {krit }}$ abgeschreckt wird, erfolgt eine Entmischung. Mit Hilfe der Thermodynamik kann die Ursache für eine Entmischung, nämlich die sich mit der Temperatur ändernden Potentiale, beschrieben werden. Um aber zu entmischen, müssen sich die A- und B-Teilchen mikroskopisch neu anordnen, z. B. von ABAABBAB nach AAAABBBB. In der thermodynamischen Beschreibung wird nicht auf den Mechanismus und die Kinetik der Neuanordnung eingegangen. Selbst wenn das Phänomen der Entmischung durch die Thermodynamik schon seit langem beschrieben werden kann, wird aktuell immer noch über mögliche Mechanismen und kinetische Konzepte diskutiert: Welche Übergangszustände existieren, warum existieren sie, und welcher Natur sind sie?

In der Vergangenheit war das Interesse an Entmischungsreaktionen aus technologischen Gründen hauptsächlich auf metallische Legierungen fokussiert. Durch Entmischungsreaktionen bilden sich Ausscheidungen, die durch ihre Größe, Anordnung und Zusammensetzung die Eigenschaften der Legierung z.T. erheblich beeinflussen. Mit dem zunehmenden Einsatz von Kunststoffmaterialien wird versucht, auch deren Eigenschaften den Einsatzbereichen anzupassen, so daß u.a. Entmischungsreaktionen in Kunststoffen eingehender untersucht werden. Es liegt nun die Vermutung nahe, daß wenn Entmischungsreaktionen täglich in technologischen Prozessen Verwendung finden, sie vollständig verstanden sind. Bei der Nutzung der Entmischungsreaktion liegt aber das Augenmerk auf dem Endzustand einer Legierung mit ihren Eigenschaften, und der Weg dorthin ist zunächst von sekundärem Interesse. Ist aber eine Verbesserung oder ein Anpassung von Materialeigenschaften gewünscht, ist es nur von Vorteil, wenn Mechanismen und Faktoren, die diese beeinflussen, bekannt sind, um so zielgerichtet arbeiten zu können.

In dieser Arbeit soll der Entmischungsmechanismus von Silber-Natriumchlorid untersucht werden. Quasibinäre Ionenkristalle wie $\mathrm{Ag}_{x} N a_{1-x} \mathrm{Cl}$ unterscheiden sich grundlegend von metallischen Systemen oder Polymersystemen dadurch, daß das Anionengitter sich nicht während der Entmischung ändert. Das Anionengitter bildet eine konstante Matrix in der die Entmischung erfolgt. Weiterhin treten in Ionenkristallen langreichweitige Coulomb-Wechselwirkungen auf, durch die Ionen über Distanzen interagieren können. Es bieten sich so die strukturell einfachen Silber-Alkalihalogenide als Modellsysteme für die Untersuchung der Entmischung in Ionenkristallen an. Um Entmischungsreaktionen experimentell verfolgen zu können, wird eine Methode benötigt, mit der die sich ändernde Atomanordnung zeitlich verfolgen läßt, also eine zeitaufgelöste strukturelle und chemische Analysemethode. Die Methoden der Wahl sind für eine derartige Fragestellungen Streumethoden. Mit ihnen ist es zum einen möglich die Anordnung von Atomen in Kristallen zu bestimmen, zum anderen können mesoskopische 

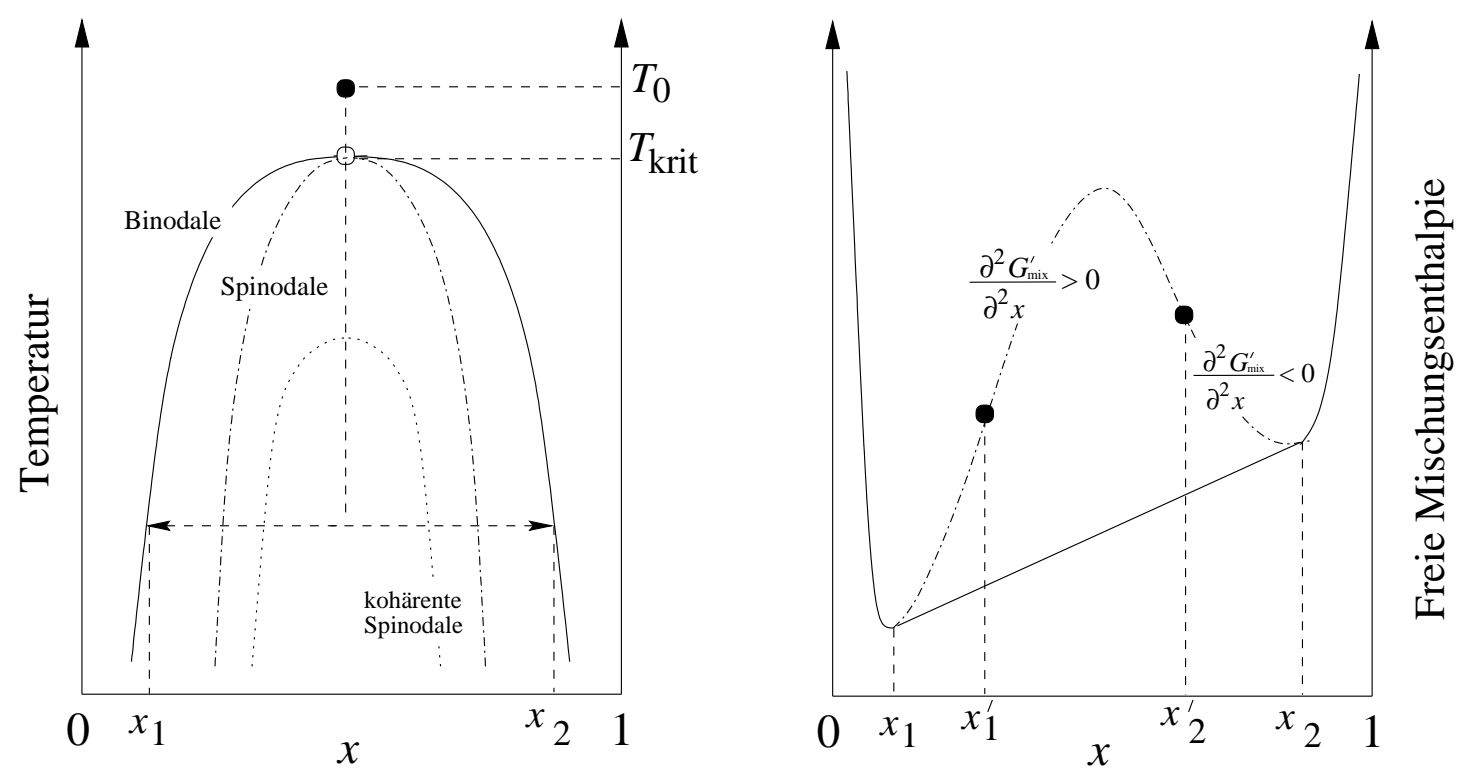

Abbildung 1.1: Zum Zusammenhang von Phasendiagramm und freier Enthalpie. Links ist die Mischungslücke eines binären Systems mit der Binodalen, der Spinodalen und der kohärenten Spinodalen gezeigt. Unterhalb der (kohärenten) Spinodalen liegt das instabile Gebiet und zwischen der Binodalen und (kohärenten) Spinodalen das metastabile Gebiet. Rechts ist die freie Mischungsenthalpie $G(x)$ für eine Temperatur unterhalb von $T_{\text {krit }}$ als durchgezogene Linie gezeigt. Die gestrichelte Linie ist $G^{\prime}$ mit den beiden Wendepunkten $\partial^{2} G^{\prime} / \partial x^{2}=0$ bei $x_{1}^{\prime}$ und $x_{2}^{\prime}$.

Strukturen wie Ausscheidungsmuster analysiert werden. Inelastische Streumethoden liefern Informationen über Energiezustände in den Kristallen. Mit der inelastischen Neutronenstreuung sind Gitterschwingungen experimentell zugänglich, die die interatomaren Wechselwirkungen widerspiegeln. Durch die geschickte Kombination der Streumethoden können komplementäre Information gesammelt werden, die zusammen ein geschlossenes Bild von Entmischungsreaktionen liefern.

\subsection{Entmischungsreaktionen}

Um Entmischungsreaktionen verstehen und beschreiben zu können, ist es notwendig, Kenntnis von der Thermodynamik der Entmischungsreaktion zu haben. Das Phasendiagramm eines binären Systems mit einer Mischungslücke ist in Abbildung 1.1 gezeigt. Die Binodale trennt den homogenen Bereich des Phasendiagramms vom Zweiphasengebiet ab. Unterhalb der Binodalen ist das System nicht in jedem Verhältnis mischbar. Eine Probe mit einer mittleren Zusammensetzung $x_{1}<x_{0}<x_{2}$ entmischt bei $T<T_{\text {binodal }}$, bis im thermodynamischen Gleichgewicht zwei Phasen mit den $\mathrm{Zu}$ sammensetzungen $x_{1}$ und $x_{2}$ koexistieren.

Die thermodynamische Größe zur Beschreibung von Entmischungsreaktion ist die 
freie Enthalpie $G$. Bei ausschließlicher Betrachtung des Entmischungsprozesses kann der Verlauf der freien Mischungsenthalpie durch die Mischungsenthalpie $H_{\mathrm{mix}}$, die Mischungsentropie $S_{\text {mix }}$ und die Temperatur $T$ beschrieben werden:

$$
G_{\mathrm{mix}}=H_{\mathrm{mix}}-T S_{\mathrm{mix}}
$$

Die Abhängigkeit von $G_{\text {mix }}(x)$ von der Konzentration ist in Abbildung 1.1 rechts dargestellt und kann anhand von Modellen, wie z. B. dem Bragg-Willams Modell ${ }^{1}$, berechnet werden. Für eine spontane Entmischung muß die Änderung der freien Mischungsenthalpie kleiner Null sein: $\Delta G<0$. Die Änderung der Mischungsentropie $\Delta S_{\text {mix }}$ beim Mischen und die Temperatur sind immer positiv, so daß der Entropieterm $-T \Delta S_{\text {mix }}$ stets negativ ist. Es ist demzufolge die Änderung der Mischungsenthalpie $\Delta H_{\text {mix }}$, in der die Wechselwirkungen der Mischungskomponenten zusammengefaßt werden, die bestimmt ob ein System mischbar ist oder nicht. Sind die Wechselwirkungen zwischen den Komponenten abstoßender Natur, ist $H_{\text {mix }}>0$ und umgekehrt. Eine Entmischungsreaktion läßt sich so durchführen, daß eine Probe mit einer mittleren Zusammensetzung $x_{1}<x_{0}<x_{2}$ von einer Temperatur oberhalb der Binodaltemperatur auf eine Temperatur unterhalb der Binodalen abgeschreckt wird. Sie entmischt, bis zwei Phasen mit den Gleichgewichtszusammensetzungen $x_{1}$ und $x_{2}$ vorliegen.

Um die Thermodynamik von Entmischungsprozessen zu beschreiben, hat es sich als ein sehr gutes Konzept erwiesen, eine freie Enthalpie $G^{\prime}$ einer theoretischen homogenen Phase innerhalb des Zweiphasengebietes einzuführen. Deren Verlauf muß im Intervall von $x_{1}$ bis $x_{2}$ durch ein Doppelminimumpotential gekennzeichnet sein, wie in Abbildung $1.1 \mathrm{zu}$ sehen ist. Anhand von $G^{\prime}$ kann die Mischungslücke weiter unterteilt werden: Durch die Ortskurve der Wendepunkte von $G^{\prime}$, der Spinodalen, wird das Zweiphasengebiet in einen instabilen Bereich mit $\partial^{2} G^{\prime}(x) / \partial x^{2}<0$ und einen metastabilen Bereich mit $\partial^{2} G^{\prime}(x) / \partial x^{2}>0$ geteilt. Im instabilen Gebiet ist für jede noch so kleine Konzentrationsänderung die Änderung der freien Mischungsenthalpie kleiner Null. Im metastabilen Bereich hingegen ist für kleine Konzentrationsänderungen $\Delta G^{\prime}>0$, so daß diese Fluktuationen nicht stabil sind. Erst bei großen Konzentrationsänderungen wird $\Delta G^{\prime}<0$. Auf diesen Unterschied bauen die Theorien der spinodalen Entmischung und der Keimbildung und des Keimwachstums auf, um die Entmischungsreaktion im instabilen bzw. metastabilen Bereich der Mischungslücke zeitabhängig zu beschreiben.

Im metastabilen Bereich des Phasendiagramms zwischen Binodale und Spinodale müssen Konzentrationsfluktuationen sehr große Amplituden aufweisen, damit die Änderung der freien Mischungsenthalpie trotz der positiven Krümmung von $G^{\prime}$ abnimmt. Es werden dabei Zusammensetzungen erreicht, die in der Nähe der Binodalzusammensetzungen $x_{1}$ und $x_{2}$ liegen. Die Änderung der freien Enthalpie setzt sich aus der Änderung der freien Enthalpiedichte $g(\mathbf{r})$ im entmischenden Volumen $V$ und einem Grenz-

\footnotetext{
${ }^{1}$ siehe z.B. [Putnis 1993]
} 

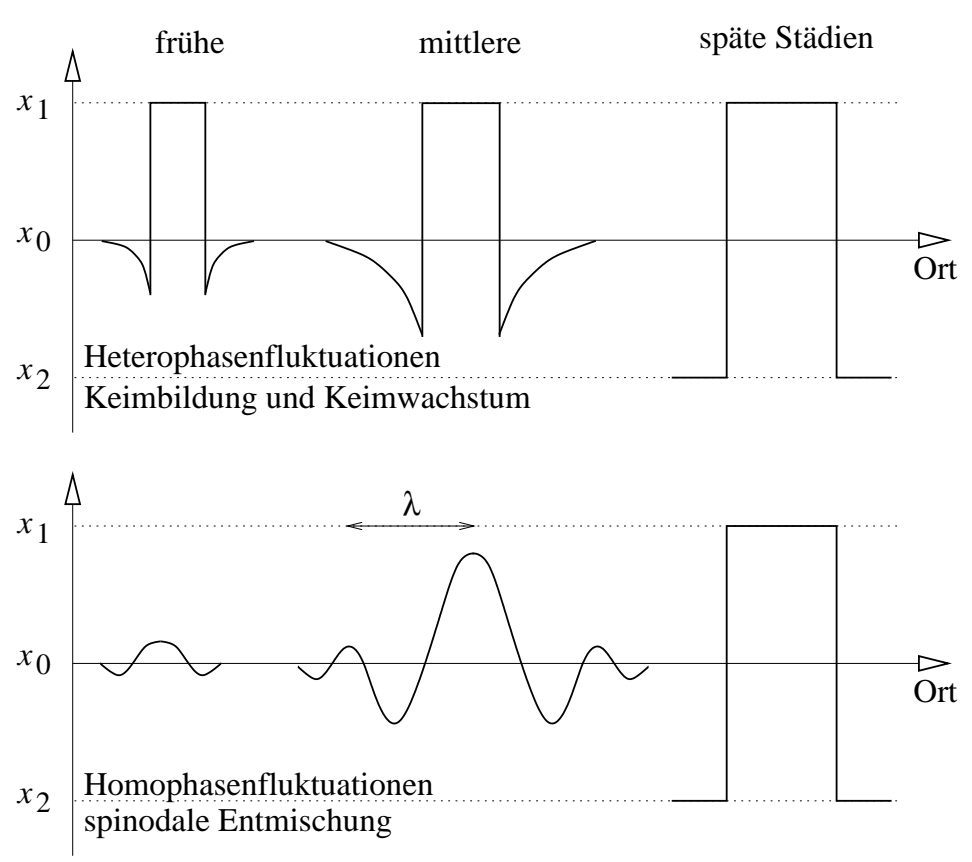

Abbildung 1.2: Hetero- und Homophasenfluktuationen

flächenenergieterm mit der Oberfläche $O$ und der Oberflächenspannung $\sigma$ zusammen:

$$
\Delta G=\Delta G_{V}+\Delta G_{O}=\Delta g(\mathbf{r}) \cdot V+O \cdot \sigma
$$

Bei einer spontanen Entmischung ist $\Delta G_{V}<0$ und $\Delta G_{O}>0$. Bei einer kugelförmigen Ausscheidung mit dem Radius $R$ ist $\Delta G_{V} \propto R^{3}$ und $\Delta G_{O} \propto R^{2}$, so daß es einen kritischen Radius gibt. Erst bei Radien, die größer als der kritische Keimbildungsradius $R_{\text {krit }}$ sind, wird $\Delta G<0$. Der Keim ist dann stabil und kann wachsen. Abbildung 1.2 zeigt eine solche Heterophasenfluktuation: In den frühen Stadien bildet sich aufgrund der großen lokalen Fluktuationsamplitude der Keim, dessen Zusammensetzung in der Nähe von $x_{1}$ liegt. Um den Keim bildet sich infolge der Massenerhaltung eine Verarmungszone, in der $x_{\text {Verarmungszone }}<x_{0}$ ist. Zwischen dem Keim und der Verarmungszone liegt eine definierte Grenzschicht. Während der mittleren Stadien wachsen Keim und Verarmungszone, wobei die Zusammensetzung der Verarmungszone sich $x_{2}$ bzw. $x_{1}$ annähert und diese Konzentration in den späten Stadien erreicht. Die Bildung von einzelnen Keimen ist unkorreliert. Der räumliche Einfluß eines einzelnen Keims begrenzt sich auf die ihn umgebende Verarmungszone, weil die Entmischung sich nur auf diesen Bereich beschränkt ${ }^{2}$. Dieser Mechanismus wird Mechanismus der Keimbildung

\footnotetext{
${ }^{2}$ Eine Ausnahme bilden z.B. Systeme, in denen große Verzerrungsenergien auftreten [Doi u. a. 1984]. In diesen Fällen kann es thermodynamisch günstiger sein, wenn ein Keim sich nach Erreichen einer weiteren kritischen Größe wieder in kleinere Keime aufspaltet.
} 
und des Keimwachstums ${ }^{3}$ genannt. In den späten Stadien der Entmischung kommt es zu Umlösungsprozessen, der sogenannten Oswaldreifung. Um die Grenzflächenenergie in der Probe zu minimieren, wachsen große Ausscheidungen an, während sich kleine Ausscheidungen auflösen. Bei der Oswaldreifung nimmt der mittlere Abstand zwischen den Ausscheidungen proportional zu $t^{-1 / 3}$ ab [Lifshitz und Slyozov 1961].

Im instabilen Bereich des Phasendiagramms führen bereits kleinste Fluktuationen, sogenannte Homophasenfluktuationen, zu einer Erniedrigung der freien Enthalpie. Abbildung 1.2 zeigt einen Ausschnitt einer Homophasenfluktuation. Wie im Falle der Keimbildung kommt es zu einer lokalen Anreicherung einer Komponente, $x(r)>x_{0}$, und zu einer Anreicherung der zweiten Komponente in der direkten Umgebung, $x(r+$ $\Delta r)<x_{0}$. Im Unterschied zur Keimbildung ist die Fluktuation kein lokaler Effekt, sondern ist von langreichweitiger periodischer Natur. Die Fluktuationsamplitude wächst wellenförmig und kontinuierlich an und breitet sich räumlich aus, weil die Zwischenzustände nicht durch eine Energiebarriere gehemmt sind. Die Amplitude der Konzentrationswelle hat einen charakteristischen periodischen Abstand $\lambda$ : Es kommt zu einem korrelierten räumlichen Konzentrationsprofil. Diesen Entmischungsmechanismus nennt man Mechanismus der spinodalen Entmischung, deren grundlegende Beschreibung auf den Arbeiten von Cahn, Hilliard und Cook [Cahn und Hilliard 1958, Cahn und Hilliard 1959a, Cahn und Hilliard 1959b, Cahn 1961, Cahn 1962, Cahn 1966, Cook 1969, Cook 1970] basiert. Nach Cahn [Cahn 1961] ergibt sich die freie Enthalpie aus der lokalen freien Enthalpiedichte $g(x(\mathbf{r}))$ und einem Gradientenenergieterm

$$
G^{\prime}=\int_{V}\left[g(x(\mathbf{r}))+\kappa(\nabla x)^{2}\right] \mathrm{d} V
$$

mit dem Parameter $\kappa$ als ein Maß für die Verzerrungsenergie, die durch den Konzentrationsgradienten hervorgerufen wird. Im Bereich einer hinreichend kleinen Umgebung der mittleren Zusammensetzung kann $g(x(\mathbf{r}))$ durch die ersten Glieder einer TaylorReihe hinreichend genau dargestellt werden. Wird die Konzentrationsfluktuation durch ihre dominierende Fourierkomponente beschrieben, ist die Abweichung von der mittleren Zusammensetzung

$$
x-x_{0}=A \cos \left(\frac{2 \pi}{\lambda} r\right) .
$$

Der Ansatz (1.4) bedeutet, daß die Konzentrationsverteilung während der Entmischung durch eine langreichweitige ebene Wellen beschrieben wird. Die Änderung der freien Enthalpie ist dann

$$
\frac{\Delta G^{\prime}}{V}=\frac{A^{2}}{4}\left[\frac{\partial^{2} g}{\partial x^{2}}+2 \kappa\left(\frac{2 \pi}{\lambda}\right)^{2}\right] .
$$

\footnotetext{
${ }^{3}$ siehe z. B. [Putnis 1993]
} 
Für nur positive anzunehmende Oberflächenspannungen, muß $\kappa \geq 0$ sein. Fluktuationen mit Wellenlängen, die kleiner als eine kritische Wellenlänge

$$
\lambda_{\text {krit. }}=\sqrt{-8 \pi \frac{\kappa}{\partial^{2} g / \partial x^{2}}}
$$

sind, wachsen nicht an, weil durch die auftretenden Verzerrungen die Mischungsenthalpie negativ wird. Je kleiner die Krümmung der lokalen freien Enthalpie und je größer die Verzerrungsenergie ist, umso größer ist $\lambda_{\text {krit. }}$. Wird die Verzerrungsenergie abgeleitet, kann die resultierende lineare Diffusionsgleichung

$$
\frac{\partial x}{\partial t}=M\left[\left(\frac{\partial^{2} g}{\partial x^{2}}+\frac{2 \eta^{2} E}{1-v}\right) \nabla^{2} x-2 \kappa \nabla^{4} x\right]
$$

( $M$ Mobilität, $E$ Elastizitätsmodul, $v$ Querkontraktionszahl, $\eta$ ist die Abhängigkeit der Gitterkonstanten $a$ von der Zusammensetzung $\eta=1 / a \cdot \partial a / \partial x$ ) gelöst werden. Es ergibt sich dann, daß Fluktuationsmoden mit $\lambda>\lambda_{\text {krit. }}$ exponentiell anwachsen. Es ist eine Wellenlänge $\lambda_{\max }=\lambda_{\text {krit. }} / \sqrt{2}$ ausgezeichnet, für die die Konzentrationsamplitude bevorzugt anwächst, so daß sich charakteristische periodische Ausscheidungsmuster bilden.

In der bisherigen Betrachtung wurde eine Verformung des Gitters durch die Entmischung außer Betracht gelassen. Die Verformung des Gitters liefert zusätzliche Beiträge zur freien Enthalpiedichte, die von der Änderung der Gitterkonstanten mit der Konzentration, der Querkontraktionszahl und dem Elastizitätsmodul abhängt:

$$
g^{\star}(x(\mathbf{r}))=g(x(\mathbf{r}))+x(\mathbf{r})^{2} \cdot\left[\left.\frac{1}{a} \frac{\partial a}{\partial x}\right|_{x=x_{0}} \cdot \frac{E}{1-v}\right]
$$

Dieser zusätzliche Beitrag verschiebt die Spinodale zu tieferen Temperaturen. Diese Kurve ist die in Abbildung1.1 gezeigte kohärente Spinodale. Es sei angemerkt, daß es sich bei Entmischungsreaktionen - im Gegensatz zur allgemeinen Erfahrung - um eine Bergaufdiffusion handelt: Um ins Gleichgewicht zu gelangen, wird ein Konzentrationsgradient nicht ab-, sondern aufgebaut.

In den späten Stadien der Entmischung führen sowohl der spinodale Entmischungsmechanismus als auch der Mechanismus der Keimbildung und des Keimwachstums zu einer Morphologie, anhand derer nicht mehr eindeutig differenziert werden kann, welcher der beiden Mechanismen zu diesem Zustand geführt hat. Dennoch wird im allgemeinen oft aufgrund einer periodischen Morphologie in den späten Entmischungstadien von einer ,spinodalen Entmischung“ gesprochen. Dies muß aber nicht in einem Zusammenhang mit dem Mechanismus der spinodalen Entmischung stehen, der wie der Mechanismus der Keimbildung und des Keimwachstums ein kinetisches Konzept darstellt. Genauso wird fälschlicherweise vom spinodalen Mechanismus gesprochen, 

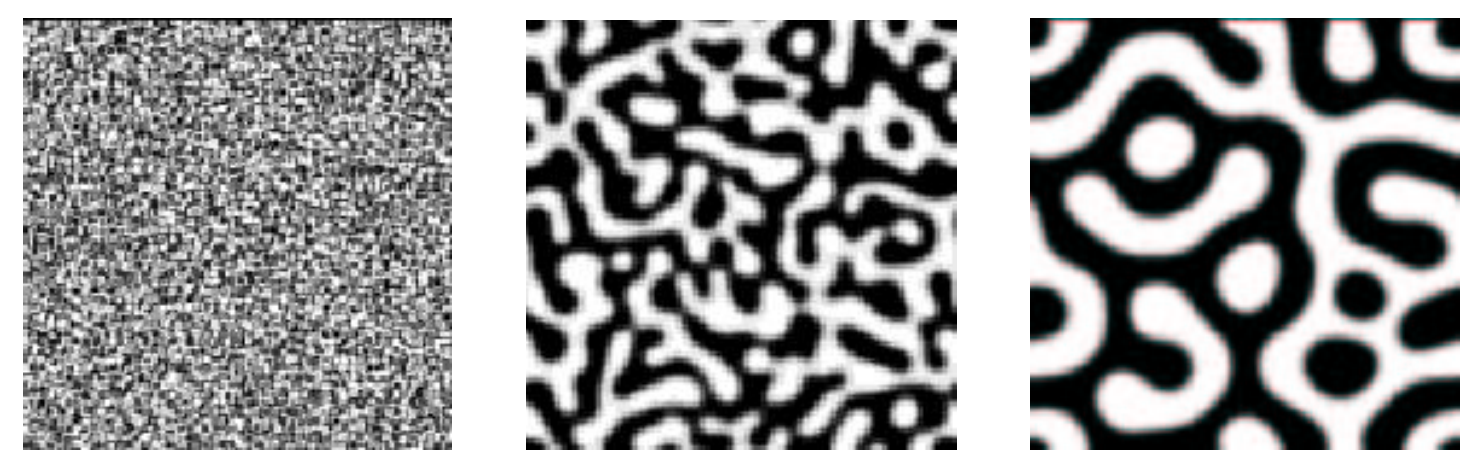

Abbildung 1.3: Momentaufnahmen der Konzentrationsverteilungen aus einer zweidimensionalen Computersimulation der spinodalen Entmischung nach Cahn und Hilliard zu drei verschiedenen Zeiten [Hawick 1991]. Aus einem homogenen (grauen) Konzentrationsfeld bilden sich zwei Phasen, die Perkolationenmuster bilden.

wenn in den späten Stadien stark vernetzte Ausscheidungsmuster, sogenannte Perkolationen [Stauffer und Aharony 1995] wie in Abbildung 1.3 vorliegen. Im Gegenzug wird vom Keimbildungsmechanismus gesprochen, wenn isolierte Ausscheidungen vorliegen. Um den spinodalen Mechanismus vom Keimbildungsmechanismus unterscheiden zu können, muß mit mikroskopischen Analysemethoden nachgewiesen werden, daß beim Abschrecken einer Probe ins instabile Gebiet des Phasendiagramms die Amplitude der Konzentrationsmodulation während der frühen Stadien kontinuierlich anwächst. Während für Polymersysteme, siehe z.B. [Madeira 1989], der Mechanismus der spinodalen Entmischung anerkannt ist, ist er in vielen Systemen noch umstritten. So konnten Inkonsistenzen zwischen unterschiedlichen Arbeiten zum System $A_{22} Z n$ auf einen Einfluß der Kühlraten [Agarwal und Hermann 1973, Bartel und Rundmann 1975, Lewandowski und Rundmann 1975], wie sie ebenfalls in $C u_{0.029} T i$ [Eckerlebe u. a. 1986] gefunden wurde, zurückgeführt werden. Neben derartigen experimentellen Problemen gibt es zudem kaum Mikroanalyseverfahren, die die Zusammensetzung im Bereich von Nanometern auflösen und selbst keinen Einfluß auf die Entmischung nehmen. Dies zeigt, wie schwierig der Nachweis zu führen ist, daß die Amplitude der Konzentrationsmodulation während der frühen Stadien kontinuierlich anwächst. Von den wenigen Nicht-Polymersystemen, für die dieser Nachweis erfolgte, seien die Systeme $\mathrm{Fe}_{29} \mathrm{Cr}_{14} \mathrm{Co}_{2} \mathrm{Al}_{0.15} \mathrm{Zr}$ [Zhu u. a. 1986], $\mathrm{FeCrCo}$ [Brenner u. a. 1984] und AlNiCo [Hutten und Grune 1986] genannt. In diesen Systemen ist eine zeitabhängige ortsauflösende Analyse einfach, weil die Kinetik auf einer Zeitskala von Stunden bis Tagen abläuft.

Eine kritische Betrachtung der rein thermodynamischen Unterscheidungskriterien ist angebracht. Das Konzept der freie Enthalpie einer homogenen Phase im Zweiphasengebiet ist z.T. einer deutlichen Kritik ausgesetzt [Binder 1986]: 
Now the assumption of double-well free energies $F^{\prime}(\phi)$ is so common practice that many workers consider this notation of metastable and unstable branches of a free energie density as a fundamental concept of gerneral validity. However, a thermodynamic potential such as the free energy $F(\phi)$ is well-defined in thermal equilibrium only, and must be a convex form of $\phi$ : this fact excludes multiple minima! Equilibrium statistical mechanics hence should not yield any branches of $\mathcal{F}(\phi)$ lying above the double-tangent construction, and, in fact, it does not: such branches really are only artefacs of mean-field approximation; the interpretation of these branches in terms of metastable and unstable states really is a kind of "folklore" lacking any fundamental justification by statistical mechanics.

Darüber hinaus kann die Kontinuumsbeschreibung nicht auf die atomare Größenordnung übertragen werden. Die Bereiche müssen zur Bestimmung der lokalen Enthalpiedichte zum einen groß genug sein, um eine mittlere lokale Konzentration definieren zu können. Zum anderen müssen sie klein gegen die Längenskala der Entmischung sein. Des weiteren ist kein diskontinuierlicher Wechsel des Entmischungsmechanismus an der Spinodalen zu erwarten. So divergiert zum Beispiel die Modulationswellenlänge $\lim _{x \rightarrow x_{1,2}^{\prime}} \lambda=\infty$ an der Spinodalen. So wurde und wird die Theorie der spinodalen Entmischung durch nichtlineare Anteile erweitert bzw. versucht, beide Konzepte durch clusterdynamische Ansätze zu vereinheitlichen ${ }^{4}$.

\subsection{Gitterdynamische Grundlagen}

Mit der Änderung der lokalen Zusammensetzung ändern sich zeitgleich die interatomaren Wechselwirkungen. Anstelle einer chemischen Mikroanalyse können somit lokal bestimmte Eigenschaften wie Phononen zur Charakterisierung herangezogen werden, um eine Änderung der lokalen Zusammensetzung zu verfolgen. Phononen spiegeln direkt die interatomaren Wechselwirkungen wider. Die Auslenkung der Atome von ihrer Ruhelage sind in der Größenordnung von $10^{-1} \AA$ und damit viel kleiner als die Längenskala einer Entmischung.

Um die Dynamik von Kristallen zu beschreiben, wird zunächst vom Gleichgewichtszustand ausgegangen. Im Gleichgewicht sitzt jedes Atom $\kappa$ am Ort $\mathbf{r}$ in der Elementarzelle $\ell$ kräftefrei auf seiner Ruhelage $\mathbf{r}_{\mathrm{\kappa} \ell}$. Die potentielle Energie $V$ ist in Abhängigkeit von den Atomlagen:

$$
V=V\left(\mathbf{r}_{11}, \mathbf{r}_{21}, \ldots, \mathbf{r}_{12}, \ldots\right)
$$

Da $V$ durch die interatomaren Wechselwirkungen gegeben ist, kann sie durch Modelle für diese Wechselwirkungen berechnet werden. Die Wechselwirkungen können in

\footnotetext{
${ }^{4}$ siehe hierzu u.a. [Langer 1971, Langer u. a. 1975, Binder und Stauffer 1974, Binder u. a. 1978]
} 
elektrostatische, kurzreichweitige abstoßende und dispersive Anteile aufgeteilt werden.

Der größte Anteil der elektrostatischen Wechselwirkungen in Ionenkristallen ist die Coulombenergie. Die Coulombenergie in kubisch-flächenzentrierten Systeme berechnet sich dann zu [Maier 2000]

$$
V=\frac{e^{2}}{4 \pi \varepsilon_{0}} \cdot \sum_{\kappa \ell \neq \kappa^{\prime} \ell^{\prime}} \frac{1}{r_{\kappa \ell, \kappa^{\prime} \ell^{\prime}}}=\frac{e^{2}}{4 \pi \varepsilon_{0}} \cdot \frac{N_{\mathrm{A}} \alpha}{d} .
$$

Für die Natriumchloridstruktur ist die Madelungkonstante $\alpha=1.74756$, und bei einer Gitterkonstanten $a$ ist $d=a / 2$. In Ionenkristallen muß es aber des Weiteren eine abstoßende Wechselwirkung zwischen den Ionen geben, da die Coulombenergie zwischen unterschiedlich geladenen Teilchen rein attraktiv ist. Wird der Abstand zwischen zwei unterschiedlich geladenen Ionen zu klein, nimmt der Anteil repulsiver Wechselwirkungen zwischen den Elektronenhüllen der Ionen erheblich zu. Diese kurzreichweitige abstoßende Wirkung kann durch das Born-Mayer Potential

$$
V=A \cdot \mathrm{e}^{-r / B}
$$

mit den Konstanten $A$ und $B$ parametrisiert werden. Die Kombination von Coulombund Born-Mayer-Potential wird Rigid-Ion-Modell (starres Ionen Modell) genannt.

Ionen sind polarisierbar, dem aber im Rigid-Ion-Modell nicht Rechnung getragen wird. Die Polarisierbarkeit von Ionen wird im sogenannten Schalenmodell berücksichtigt: Die Ionen werden in einen Kern, der sich aus dem Atomkern und den inneren Elektronenschalen zusammensetzt, und die äußere Elektronenschale aufgeteilt. Schale und Kern tragen eine Ladung, deren Summe gleich der Ionenladung ist. Zwischen zwei benachbarten Ionen werden longitudinale und transversale Federpotentiale zwischen den Kernen und Schalen angesetzt, wie Abbildung 1.4 zeigt.

Bei kleinen Auslenkungen der Atome aus ihrer Ruhelage kann das Potential durch die Taylorreihe

$$
\begin{aligned}
V & =\underbrace{V_{0}}_{\equiv 0}+\underbrace{\left.\sum_{\kappa \ell} \sum_{\alpha=1}^{3} \frac{\partial V(\kappa \ell, \kappa \ell)}{\partial \mathbf{u}_{\kappa \ell}^{\alpha}}\right|_{\mathbf{r}_{\kappa \ell}^{\circ}} ^{o}}_{=0} \cdot \mathbf{u}^{\alpha}(\kappa \ell) \\
+ & \underbrace{\left.\frac{1}{2} \sum_{\kappa \ell} \sum_{\kappa^{\prime} \ell^{\prime}} \sum_{\alpha=1}^{3} \sum_{\beta=1}^{3} \mathbf{u}_{\alpha}(\kappa \ell) \frac{\partial^{2} V\left(\kappa \ell, \kappa^{\prime} \ell^{\prime}\right)}{\partial \mathbf{u}_{\kappa}^{\alpha} \partial \mathbf{u}_{\kappa^{\prime} \ell}^{\beta}}\right|_{\mathbf{r}=\mathbf{r}_{\kappa \ell}^{\circ}} \mathbf{u}_{\beta}\left(\kappa^{\prime} \ell^{\prime}\right)}_{\text {harmonische Näherung } V^{I I}\left(\kappa \ell, \kappa^{\prime} \ell^{\prime}\right)=\partial^{2} V\left(\kappa \ell, \kappa^{\prime} \ell^{\prime}\right) / \partial \mathbf{u}_{\kappa} \ell^{\alpha} \mathbf{u}_{\mathrm{K}^{\prime} \ell^{\prime}}^{\beta}}+\ldots
\end{aligned}
$$

beschrieben werden. Es indizieren $\alpha$ und $\beta$ die drei Raumrichtungen. Das Gleichgewichtspotential $V_{0}$ kann auf $V_{0} \equiv 0$ gesetzt werden, da nur Abweichungen vom Gleich- 


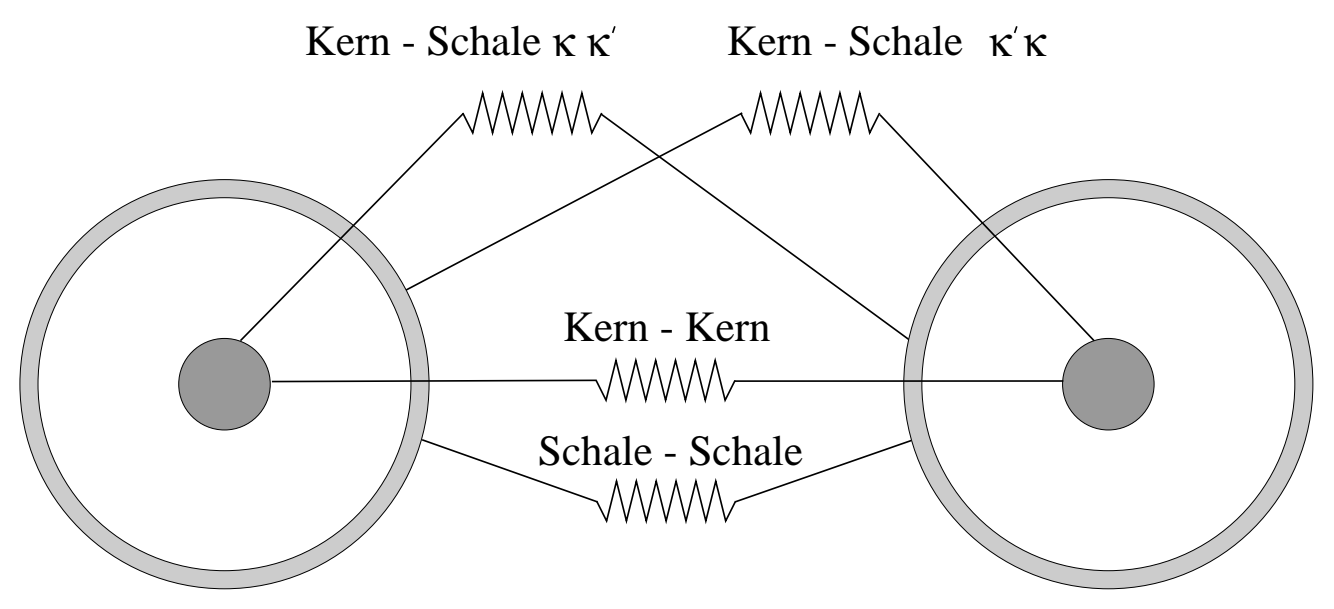

Abbildung 1.4: Die Wechselwirkungen zwischen zwei Atomen $\kappa$ und $\kappa^{\prime}$ im Schalenmodell

gewicht interessieren. Der zweite Term von Gleichung (1.12) muß aufgrund der Gleichgewichtsbedingung Null sein. Wird ein Atom aus seiner Ruhelage verschoben, resultiert eine auf alle anderen Atome wirkende Kraft, die die Atome von ihrer Ruhelage auslenkt. Diese Information ist in der harmonischen Näherung im zweiten Term enthalten. In Matritzenschreibweise ist die potentielle Energie durch

$$
\begin{aligned}
V & =-\frac{1}{2} \sum_{\kappa \ell} \sum_{\kappa^{\prime} \ell^{\prime}} \mathbf{u}_{\kappa \ell}^{\mathrm{T}} \underbrace{\left(\begin{array}{ccc}
V_{11}^{I I}(\kappa \ell, \kappa \ell) & V_{12}^{I I}\left(\kappa \ell, \kappa^{\prime} \ell^{\prime}\right) & V_{13}^{I I}\left(\kappa \ell, \kappa^{\prime} \ell^{\prime}\right) \\
V_{21}^{I I}\left(\kappa \ell, \kappa^{\prime} \ell^{\prime}\right) & V_{22}^{I I}\left(\kappa \ell, \kappa^{\prime} \ell^{\prime}\right) & V_{23}^{I I}\left(\kappa \ell, \kappa^{\prime} \ell^{\prime}\right) \\
V_{31}^{I I}\left(\kappa \ell, \kappa^{\prime} \ell^{\prime}\right) & V_{32}^{I I}\left(\kappa \ell, \kappa^{\prime} \ell^{\prime}\right) & V_{33}^{I I}\left(\kappa \ell, \kappa^{\prime} \ell^{\prime}\right)
\end{array}\right)}_{\text {Matrix der Kraftkonstanten }} \mathbf{u}_{\kappa^{\prime} \ell^{\prime}} \\
& =-\frac{1}{2} \sum_{\kappa \ell} \sum_{\kappa^{\prime} \ell^{\prime}} \mathbf{u}_{\kappa \ell}^{\mathrm{T}} \mathbf{V}\left(\kappa \ell, \kappa^{\prime} \ell^{\prime}\right) \mathbf{u}_{\kappa \ell}
\end{aligned}
$$

gegeben, und das zu lösende System von Bewegungsgleichungen lautet

$$
m_{\kappa} \frac{\mathrm{d}^{2} \mathbf{u}_{\kappa \ell}(t)}{\mathrm{d} t^{2}}=-\sum_{\kappa \ell} \mathbf{V}(\kappa \ell, \kappa \ell) \mathbf{u}_{\kappa \ell}(t),
$$

wobei $m_{\kappa}$ die Masse des Atoms am Ort $\mathbf{r}_{\kappa \ell}$ bezeichnet. Als Lösungsansatz werden ebene Wellen

$$
\mathbf{u}_{\kappa \ell}(\mathbf{q}, j)=\frac{1}{\sqrt{N m_{\kappa}}} \mathbf{e}_{\kappa}(\mathbf{q}, j) \mathrm{e}^{l\left(\mathbf{q r}_{\ell} \pm \omega_{j}(\mathbf{q}) t\right)}
$$

gewählt. $N$ ist die Anzahl aller Teilchen. Diese Wellen, Phononen, werden durch einen Wellenvektor q, eine Polarisation e und eine Frequenz $\omega_{j}$ charakterisiert. Der Index $j$ indiziert die unterschiedlichen Dispersionszweige für einen Wellenvektor. Wird der 

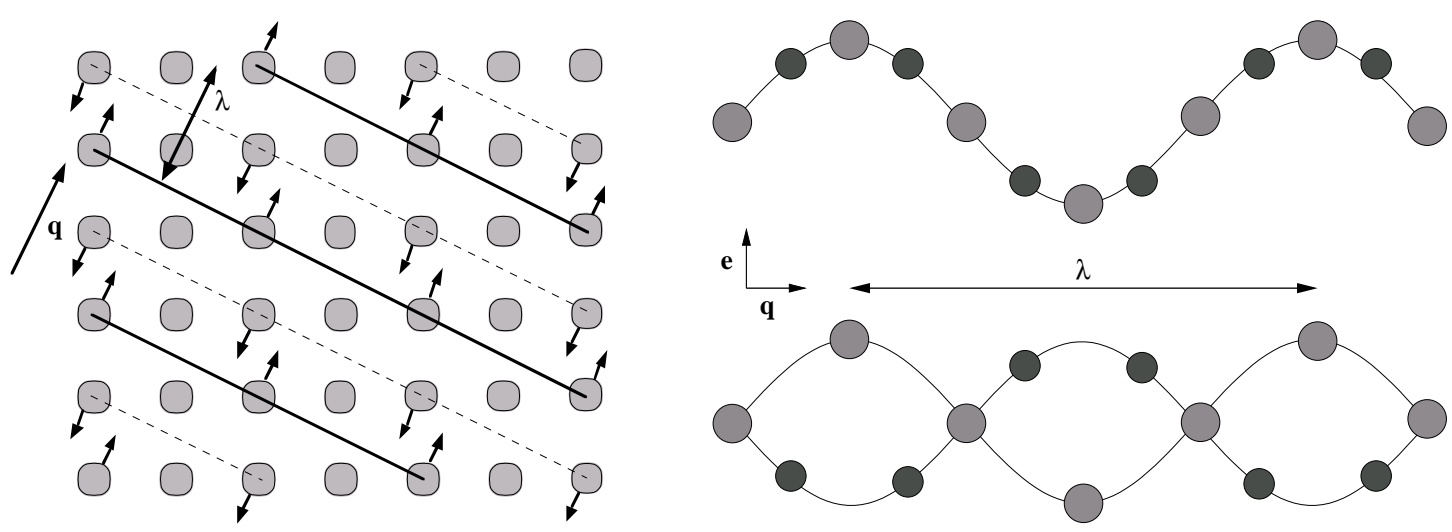

Abbildung 1.5: Die Ausbreitung einer longitudinalen Welle in einem zweidimensionalen Gitter (links), einer transversal akustischen und einer transversal optische Welle in einer linearen Kette (beide rechts)

Ansatz (1.15) in Gleichung (1.14) eingesetzt, ergibt sich

$$
\begin{aligned}
\sqrt{\frac{m_{\kappa}}{N}} \mathbf{e}_{\kappa}(\mathbf{q}, j) \omega^{2} \mathrm{e}^{l\left(\mathbf{q r}_{\ell} \pm \omega_{j}(\mathbf{q}) t\right)} & =-\sum_{\kappa^{\prime} \ell^{\prime}} \mathbf{V}\left(\kappa \ell, \kappa^{\prime} \ell^{\prime}\right) \frac{1}{\sqrt{N m_{\kappa^{\prime}}}} \mathbf{e}_{\mathcal{K}^{\prime}}(\mathbf{q}, j) \mathrm{e}^{l\left(\mathbf{q r}_{\ell^{\prime}} \pm \omega(\mathbf{q}, j) t\right)} \\
\Leftrightarrow \sqrt{\frac{m_{\kappa}}{N}} \mathbf{e}_{\kappa}(\mathbf{q}, j) \omega^{2} \mathrm{e}^{\imath \mathbf{q} \mathbf{r}_{\ell}} & =-\sum_{\kappa^{\prime}} \frac{1}{\sqrt{m_{\kappa} m_{\kappa^{\prime}}}} \sum_{\ell^{\prime}}\left[\mathbf{V}\left(\kappa \ell, \kappa^{\prime} \ell^{\prime}\right) \mathrm{e}^{\left.\imath \mathbf{q}\left(\mathbf{r}_{\ell^{\prime}}-\mathbf{r}_{\ell}\right)\right] \cdot \mathbf{e}_{\mathrm{K}^{\prime}}(\mathbf{q}, j)}\right. \\
\Leftrightarrow \omega^{2}(\mathbf{q}, j) & =\sum_{\kappa} \mathbf{e}_{\kappa}^{\mathrm{T}}(\mathbf{q}, j) \sum_{\kappa^{\prime}} \underline{\underline{\mathrm{D}}}\left(\kappa \kappa^{\prime}\right) \mathbf{e}_{\kappa^{\prime}}(\mathbf{q}, j) .
\end{aligned}
$$

Die Lösung ${ }^{5}$ des Differentialgleichungssystem (1.16) ergibt den Zusammenhang von Wellenvektor und Frequenz, die Dispersionsrelation oder kurz Dispersion erhalten. In der Regel wird sie für Wellenvektoren parallel zu den Hauptsymmetrierichtungen als Auftragung $\omega$ oder v vs. q dargestellt. Für kubische Gitter sind Hauptsymmetrierichtungen die [ $\left[\begin{array}{lll}1 & 0 & 0\end{array}\right]$-, $\left[\begin{array}{lll}0 & 1 & 1\end{array}\right]$ - und [ $\left[\begin{array}{lll}1 & 1 & 1\end{array}\right]$-Richtung. Es können für $\mathbf{q} \rightarrow 0$ zwischen vier Typen von ebenen Wellen unterschieden werden: longitudinale Moden, bei denen $\mathbf{e} \| \mathbf{q}$ ist; transversale Moden, bei denen $\mathbf{e} \perp \mathbf{q}$; akustische Moden, bei denen die Atome gleichphasig schwingen; und optische Moden, bei denen Atome unterschiedlichen Typs gegenphasig schwingen. Die Kombination ergibt longitudinal akustische (LA), longitudinal optische (LO) und transversal akustische (TA) bzw. transversal optische (TO) Wellen, vgl. Abbildung 1.5. Es gibt drei akustische und $3 \cdot N-3$ optische Moden, wenn $N$ die Anzahl der Atome in der primitiven Zelle ist.

Im Rahmen dieser Arbeit ist der Grenzfall großer Wellenlängen, also kleinen Wellenvektoren, von besonderem Interesse. Akustische Gitterschwingungen mit großen Wel-

\footnotetext{
${ }^{5}$ Für eine detaillierte Darstellung der Lösung sei auf [Dove 1993] und insbesondere auf [Eckold 1994, Eckold 1992, Elter und Eckold 2000] verwiesen.
} 
lenlängen entsprechen Schallwellen. Am Gammapunkt $\mathbf{q}=\left(\begin{array}{lll}0 & 0 & 0\end{array}\right)$ gilt für die akustischen Moden, daß $\omega=0$, und für Schallwellen ist die Ausbreitungsgeschwindigkeit

$$
v_{\text {Schall }}=\frac{\omega}{q} .
$$

Die Steigung der akustischen Dispersionszweige für $q \rightarrow 0$ ist infolgedessen die entsprechende Schallgeschwindigkeit. Diese ergibt sich aber auch aus der Massendichte und den elastischen Konstanten. In kubischen Systemen sind die Schallgeschwindigkeiten $v$ mit den elastischen Konstanten $c_{11}, c_{12}$ und $c_{44}$ wie folgt mit den Ausbreitungsrichtungen verknüpft:

parallel zu $\left[\begin{array}{lll}1 & 0 & 0\end{array}\right]$

$$
\begin{aligned}
\mathbf{e} \| \mathbf{q} & =\left[\begin{array}{lll}
1 & 0 & 0
\end{array}\right] & v & =\sqrt{\frac{c_{11}}{\rho}} \\
\mathbf{e} \perp \mathbf{q} & =\left[\begin{array}{lll}
0 & 1 & 0
\end{array}\right] & v & =\sqrt{\frac{c_{44}}{\rho}} \\
\mathbf{e} \perp \mathbf{q} & =\left[\begin{array}{lll}
0 & 0 & 1
\end{array}\right] & v & =\sqrt{\frac{c_{44}}{\rho}}
\end{aligned}
$$

parallel zu $\left[\begin{array}{lll}1 & 1 & 0\end{array}\right]$

$$
\begin{array}{rlrl}
\mathbf{e} \| \mathbf{q} & =\left[\begin{array}{lll}
0 & 1 & 1
\end{array}\right] & v & =\sqrt{\frac{c_{11}+c_{12}+2 c_{44}}{2 \rho}} \\
\mathbf{e} \perp \mathbf{q}=\left[\begin{array}{lll}
0 & 1 & 1
\end{array}\right] & v & =\sqrt{\frac{c_{11}-c_{12}}{2 \rho}} \\
\mathbf{e} \perp \mathbf{q}=\left[\begin{array}{lll}
1 & 0 & 0
\end{array}\right] & v & =\sqrt{\frac{c_{44}}{\rho}}
\end{array}
$$

parallel zu [ $\left[\begin{array}{lll}1 & 1 & 1\end{array}\right]$

$$
\begin{aligned}
\mathbf{e} \| \mathbf{q}=\left[\begin{array}{lll}
1 & 1 & 1
\end{array}\right] & v=\sqrt{\frac{c_{11}+2 c_{12}+4 c_{44}}{3 \rho}} \\
\mathbf{e} \perp \mathbf{q}=\left[\begin{array}{lll}
2 & 1 & 1
\end{array}\right] & v=\sqrt{\frac{c_{11}-c_{12}+c_{44}}{3 \rho}} \\
\mathbf{e} \perp \mathbf{q}=\left[\begin{array}{lll}
0 & 1 & 1
\end{array}\right] & v=\sqrt{\frac{c_{11}-c_{12}+c_{44}}{3 \rho}}
\end{aligned}
$$

Die elastischen Konstanten spiegeln dabei in kubischen Systemen folgende Eigenschaften wider:

$c_{11}$ gibt an, welche Spannung ausgeübt werden muß, um eine Dehnung parallel zur Spannung zu produzieren.

$c_{12}$ gibt an, welche Spannung ausgeübt werden muß, um eine Dehnung senkrecht zur Spannung, eine Querkontraktion, zu produzieren.

$c_{44}$ gibt an, welche Scherspannung ausgeübt werden muß, um eine Scherdeformation zu produzieren. 

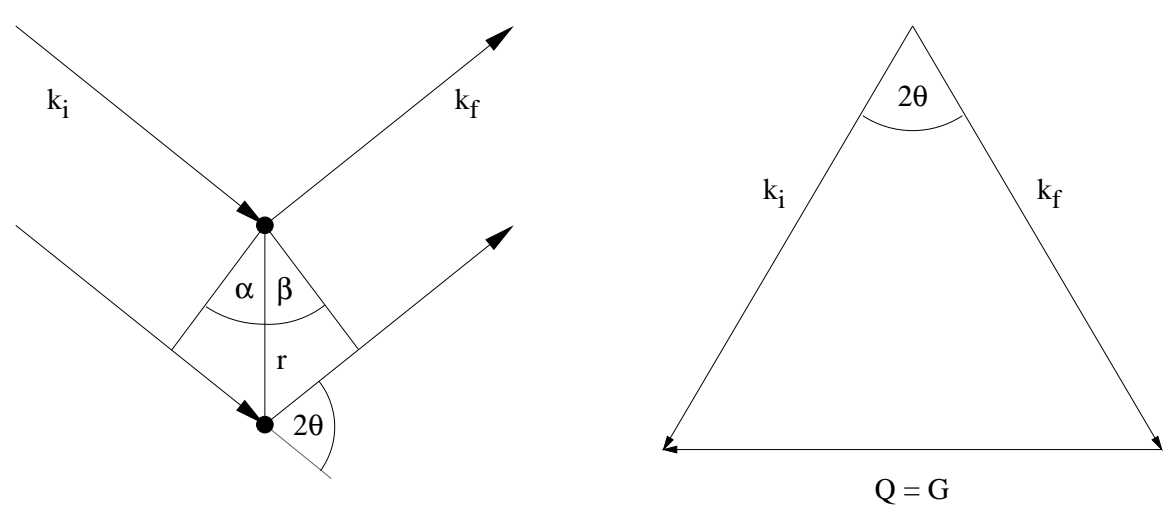

Abbildung 1.6: Zur Streugeometrie zweier Streuzentren und das Streudreieck im elastischen Fall $\left(k_{\mathrm{i}}=k_{\mathrm{f}}\right)$

\subsection{Elastische Neutronenstreuung ${ }^{6}$}

Eine Möglichkeit, mikroskopische Strukturen auf der atomaren Größenskala aufzulösen, ist die Neutronenstreuung. Dabei wird ausgenutzt, daß die Wellenlängen von Neutronen, $\lambda \approx 10^{-10} \mathrm{~m}$, zum einen die gleiche Größenordnung wie die interatomaren Abstände besitzen, zum anderen jedoch um Größenordnungen größer als Atomkerne sind, an denen sie gestreut werden. Damit können Atomkerne als punktförmig angenommen werden, und es kann für die Neutronenstreuung das Huygensche Prinzip angewendet werden. Um das Streubild zweier punktförmiger Teilchen wie in Abbildung 1.6 links beschreiben zu können, werden die Streulängen $b$ der Teilchen, deren Abstand $\mathbf{r}$ zueinander und die Wellenvektoren der einfallenden und ausfallenden Welle $\mathbf{k}_{\mathrm{i}}$ und $\mathbf{k}_{\mathrm{f}}{ }^{7}$ benötigt. Der Gangunterschied von $\mathbf{k}_{\mathrm{i}}$ und $\mathbf{k}_{\mathrm{f}}$ ist

$$
\left[k_{\mathrm{i}} \cdot r \cdot \cos (\alpha)-k_{\mathrm{f}} \cdot r \cdot \cos (\beta)\right]-\left(\omega_{i}-\omega_{f}\right) \cdot t=\left[\left(\mathbf{k}_{\mathrm{i}}-\mathbf{k}_{\mathrm{f}}\right) \cdot \mathbf{r}\right]-\omega t=\mathbf{Q} \cdot \mathbf{r}-\omega t .
$$

Es ist $t$ die Zeit, die die einfallende Welle braucht, um vom einen Streuzentrum zum anderen zu gelangen, und $\omega$ ist die Änderung der Kreisfrequenz des Neutrons $\omega=$ $\omega_{\mathrm{i}}-\omega_{\mathrm{f}}$. Dabei muß die Impuls- und Energieerhaltung

$$
\hbar \mathbf{Q}=\hbar\left(\mathbf{k}_{\mathrm{i}}-\mathbf{k}_{\mathrm{f}}\right) \quad \text { und } \quad E_{\mathrm{i}}-E_{\mathrm{f}}=\frac{\hbar}{2 m_{\mathrm{n}}}\left(k_{\mathrm{i}}^{2}-k_{\mathrm{f}}^{2}\right)
$$

gelten. Die Differenz vom ein- und ausfallenden Wellenvektor $\mathbf{Q}$ ist der sogenannte Streuvektor oder Wellenvektorübertrag. Im elastischen Fall $k_{i}=k_{f}$ findet eine positive

\footnotetext{
${ }^{6}$ Zur Neutronenstreuung siehe z.B. [Squires 1978, Dorner 1982, Lovesey 1984, Sturm 1996, Brückel u. a. 2000, Scherm 1972]

${ }^{7}$ Die Indizes stehen für incident und final.
} 
Interferenz statt, wenn die Braggsche Bedingung

$$
n \cdot 2 \pi / k_{i}=2 r \sin (\theta) \quad \text { mit } \quad n \in \mathbb{N}
$$

erfüllt ist. Die Streulänge $b$ ist im Falle der Neutronenstreuung eine elementspezifische, $Q$-unabhängige Größe. Sie beschreibt das Streuvermögen eines Teilchens, das in Bezug auf die Intensität die Einheit eines Wirkungsquerschnitts besitzt. Im Falle der Röntgendiffraktion ist $b$ durch den $Q$-abhängigen Formfaktor zu ersetzen. Die für diese Arbeit wichtigsten Streulängen sind für natürliche Isotopengemische nach [Sears 1992]

$$
b_{A g}=0.592 \cdot 10^{-12} \mathrm{~cm} \quad b_{N a}=0.363 \cdot 10^{-12} \mathrm{~cm} \quad b_{C l}=0.958 \cdot 10^{-12} \mathrm{~cm} .
$$

Dieser Ansatz ist auf ein ideales, dreidimensionales Gitter aus $N$ Teilchen erweiterbar. Jedes Teilchen $\kappa$ mit der Streulänge $b_{\kappa}$ sitzt in der Elementarzelle $\ell$ am Ort $\mathbf{r}_{\kappa \ell}$. Die Amplitude der gestreuten Welle im elastischen Fall ergibt sich durch phasenrichtiges Aufsummieren aller entstehenden Streuwellen:

$$
F(\mathbf{Q})=\sum_{\kappa \ell} b_{\mathrm{\kappa}} \mathrm{e}^{l \mathbf{Q} \cdot \mathbf{r}_{\mathrm{\kappa} \ell}}
$$

Das Gitter der punktförmigen Streuzentren kann als Summe von Deltafunktionen formuliert werden,

$$
V(\mathbf{r})=\sum_{\kappa \ell} b_{\kappa} \cdot \delta\left(\mathbf{r}-\mathbf{r}_{\kappa \ell}\right)
$$

deren Fouriertransformierte

$$
\begin{aligned}
\int V(\mathbf{r}) \mathrm{e}^{l \mathbf{Q} \cdot \mathbf{r}} \mathrm{d} \mathbf{r} & =\sum_{\kappa \ell} b_{\kappa} \int \delta\left(\mathbf{r}-\mathbf{r}_{\kappa}\right) \mathrm{e}^{l \mathbf{Q} \cdot \mathbf{r}} \mathrm{d} \mathbf{r} \\
& =\sum_{\kappa \ell} b_{\kappa} \cdot \mathrm{e}^{l \mathbf{Q} \cdot \mathbf{r}_{\kappa \ell}}
\end{aligned}
$$

ist. Der Vergleich von (1.26) mit (1.24) zeigt, daß das Streubild die Fouriertransformierte der Streuzentrenanordnung ist. Sie enthält als Information die relative Lage der Streuzentren zueinander, jedoch nicht deren absolute Positionen. Wird berücksichtigt, daß die Streulänge eine komplexe Größe ist, ergibt sich für die Intensität

$$
\begin{aligned}
& |F(\mathbf{Q})|^{2}=\sum_{\kappa \ell, \kappa^{\prime} \ell^{\prime}} b_{\kappa} b_{\kappa^{\prime}}^{\star} e^{l \mathbf{Q}\left(\mathbf{r}_{\kappa}-\mathbf{r}_{\ell^{\prime} \kappa^{\prime}}\right)}=\sum_{\kappa \ell} b_{\kappa} \cdot e^{l \mathbf{Q} \cdot \mathbf{r}_{\kappa} \ell} \cdot \sum_{\ell^{\prime} \kappa^{\prime}} b_{\kappa}^{\star} \cdot \mathrm{e}^{-l \mathbf{Q} \cdot \mathbf{r}_{\ell^{\prime} \kappa^{\prime}}} \\
& =\left|\mathrm{e}^{\imath \mathbf{Q} \cdot \mathbf{r}}\right|^{2} \sum_{\kappa \kappa^{\prime}} b_{\kappa} b_{\kappa^{\prime}}^{\star}=\underbrace{|\bar{b}|^{2} \cdot\left|\mathrm{e}^{\imath \mathbf{Q} \cdot \mathbf{r}}\right|^{2}}_{\text {wenn } \mathrm{\kappa} \neq \kappa^{\prime}}+\underbrace{N \cdot \overline{|b-\bar{b}|^{2}}}_{\text {wenn } \kappa=\kappa^{\prime}} .
\end{aligned}
$$

Die Intensität setzt sich also aus einem $\mathbf{Q}$-abhängigen Term $\left(\kappa \neq \kappa^{\prime}\right)$ und einem $\mathbf{Q}$ - 
unabhängigen Term $\left(\kappa=\kappa^{\prime}\right)$ zusammen. Der erste Term wird kohärenter Streubeitrag genannt. Er enthält Informationen über die Korrelation eines Streuzentrums mit allen Streuzentren und liefert immer dann Beiträge zur Streufunktion, wenn $\mathbf{Q}$ gleich einem reziproken Gittervektor $\mathbf{G}=h \cdot \mathbf{a}^{\star}+k \cdot \mathbf{b}^{\star}+l \cdot \mathbf{c}^{\star}$ ist $^{8}$. Der zweite Term wird inkohärenter Streubeitrag genannt. Dieser enthält die Information über die Korrelation eines Streuzentrums mit sich selbst.

Im Gegensatz zu idealen Gittern sind in festen Körpern die Atome nie in Ruhe, sondern schwingen mindestens mit der Nullpunktsenergie um ihre Ruhelage $\mathbf{r}_{\kappa \ell}(t)=$ $\mathbf{R}_{\mathrm{\kappa} \ell}+\mathbf{u}_{\mathrm{\kappa} \ell}(t)$. Gleichung (1.24) geht mit dieser Erweiterung in

$$
F(\mathbf{Q}, t)=\sum_{\kappa \ell} b_{\kappa} \mathrm{e}^{l \mathbf{Q} r_{\kappa}(t)}=\sum_{\kappa \ell} b_{\kappa} \mathrm{e}^{l \mathbf{Q}\left(\mathbf{R}_{\kappa} \ell+\mathbf{u}_{\kappa}(t)\right)}
$$

über. Im zeitlichen Mittel ist

$$
\begin{aligned}
F(\mathbf{Q}) & =\sum_{\kappa \ell} b_{\kappa} \mathrm{e}^{i \mathbf{Q} \mathbf{R}_{\kappa \ell}}\left\langle\mathrm{e}^{i \mathbf{Q \mathbf { u } _ { \kappa } \ell}(t)}\right\rangle=\sum_{\kappa} b_{\kappa} \mathrm{e}^{l \mathbf{Q} \mathbf{R}_{\kappa \ell}} \mathrm{e}^{-\frac{1}{2}\left\langle\left|\mathbf{Q} \mathbf{u}_{\kappa \ell}\right|^{2}\right\rangle} \\
& =\sum_{\kappa \ell} b_{\kappa} \mathrm{e}^{l \mathbf{Q} \mathbf{R}_{\kappa \ell}-W(\mathbf{Q})}
\end{aligned}
$$

Der Korrekturfaktor $W(\mathbf{Q})$ wird als Temperaturfaktor oder Debye-Waller-Faktor bezeichnet. Er beschreibt die Intensitätsabnahme zu größeren Streuvektoren in Abhängigkeit von der mittleren Auslenkung der Atome, die mit der Temperatur zunimmt.

\subsection{Inelastische Neutronenstreuung}

Die eigentliche Stärke der Neutronenstreuung liegt in der inelastischen Neutronenstreuung, wenn also $k_{\mathrm{i}} \neq k_{\mathrm{f}}$ ist. Mit Neutronen können nicht nur mikroskopische Strukturen in atomarer Größenordnung aufgelöst werden. Sie besitzen zudem Energien in der gleichen Größenordnung wie Gitterschwingungen. In einem inelastischen Neutronenstreuexperiment treffen Neutronen mit dem Wellenvektor $\mathbf{k}_{\mathrm{i}}$ mit der Wahrscheinlichkeit $W_{\mathrm{i}}\left(\mathbf{k}_{\mathbf{i}}\right) \mathrm{d} \mathbf{k}_{\mathrm{i}}$ auf die Probe. Analog werden die von der Probe gestreuten Neutronen mit einer Wahrscheinlichkeit $W_{\mathrm{f}}\left(\mathbf{k}_{\mathrm{f}}\right) \mathrm{d} \mathbf{k}_{\mathrm{f}}$ nachgewiesen. Diese zwei Wahrscheinlichkeiten sind unabhängig von der Probe, an der die Neutronen gestreut werden. $W_{\mathrm{i}}\left(\mathbf{k}_{\mathrm{i}}\right) \mathrm{d} \mathbf{k}_{\mathrm{i}}$ ist durch die Neutronenquelle, Strahlrohre bzw. Neutronenleiter und die Spektrometerelemente vor der Probe bedingt, und $W_{\mathrm{i}}\left(\mathbf{k}_{\mathrm{f}}\right) \mathrm{d} \mathbf{k}_{\mathrm{f}}$ ist charakteristisch für die Spektrometerelemente, mit denen die gestreuten Neutronen detektieren werden. Bei der Streuung

${ }^{8}$ Die Basis des reziproken Gitters ist durch die Gitterkonstanten $\mathbf{a}$, b und $\mathbf{c}$ gegeben:

$$
\mathbf{a}^{\star}=2 \pi \frac{\mathbf{b} \times \mathbf{c}}{\mathbf{a} \cdot(\mathbf{b} \times \mathbf{c})} \quad \mathbf{b}^{\star}=2 \pi \frac{\mathbf{c} \times \mathbf{a}}{\mathbf{a} \cdot(\mathbf{b} \times \mathbf{c})} \quad \mathbf{c}^{\star}=2 \pi \frac{\mathbf{a} \times \mathbf{b}}{\mathbf{a} \cdot(\mathbf{b} \times \mathbf{c})}
$$


an der Probe kann sich der Wellenvektor von $\mathbf{k}_{\mathrm{i}}$ nach $\mathbf{k}_{\mathbf{f}}$ mit der Wahrscheinlichkeit $W\left(\mathbf{k}_{\mathrm{i}} \rightarrow \mathbf{k}_{\mathrm{f}}\right)$ ändern. Dieser Übergang hängt nur von der Probe ab. Gibt bei einem Streuprozeß ein Neutron Energie an die Probe ab $\left(\Delta E_{\text {Neutron }}<0\right)$ oder wird Energie von der Probe auf das Neutron übertragen $\left(\Delta E_{\text {Neutron }}>0\right)$, muß es in der Probe einen Energieübergang mit der gleichen Energiedifferenz geben. Die gemessene Intensität ist das Integral der Gesamtwahrscheinlichkeit über alle ein- und ausfallenden Wellenvektoren $I=\iint W_{\mathrm{i}}\left(\mathbf{k}_{\mathrm{i}}\right) W\left(\mathbf{k}_{\mathrm{i}} \rightarrow \mathbf{k}_{\mathrm{f}}\right) W_{\mathrm{f}}\left(\mathbf{k}_{\mathrm{f}}\right) \mathrm{d} \mathbf{k}_{\mathrm{i}} \mathrm{d} \mathbf{k}_{\mathrm{f}}$. Die Wahrscheinlichkeit des Übergangs $W\left(\mathbf{k}_{\mathrm{i}} \rightarrow \mathbf{k}_{\mathrm{f}}\right)$ kann mit der quantenmechanischen Störungstheorie berechnet werden. In dem aus Probe $n_{\mathrm{i}}$ und Neutron $k_{\mathrm{i}}$ bestehenden System ist die Wahrscheinlichkeit für den Übergang vom Zustand $\left|\mathbf{k}_{\mathrm{i}} n_{\mathrm{i}}\right\rangle$ in einen Zustand $\left|\mathbf{k}_{\mathrm{f}} n_{\mathrm{f}}\right\rangle$ durch Fermis goldene Regel

$$
W\left(\mathbf{k}_{\mathrm{i}} n_{\mathrm{i}} \rightarrow \mathbf{k}_{\mathrm{f}} n_{\mathrm{f}}\right) \mathrm{d} \mathbf{k}_{\mathrm{f}}=\frac{2 \pi}{\hbar}\left|\left\langle\mathbf{k}_{\mathrm{f}} n_{\mathrm{f}}|V(\mathbf{r})| \mathbf{k}_{\mathrm{i}} n_{\mathrm{i}}\right\rangle\right|^{2} \delta\left(E_{\mathrm{i}}-E_{\mathrm{f}}-\hbar \omega\right)
$$

gegeben. $V(\mathbf{r})$ ist bei der Streuung an Atomkernen das Fermi-Pseudopotential

$$
V(\mathbf{r})=\frac{2 \pi \hbar^{2}}{m_{\mathrm{n}}} \sum_{\kappa} b_{\kappa} \delta\left(\mathbf{r}-\mathbf{r}_{\kappa}\right) .
$$

Experimentell ist aber nur die Übergangswahrscheinlichkeit für ein Neutron von $\mathbf{k}_{\mathbf{i}} \rightarrow$ $\mathbf{k}_{\mathrm{f}}$ zugänglich, weil das Spektrometer keine Kenntnis von den Zuständen in der Probe hat. Diese Übergangswahrscheinlichkeit ist das Produkt aus der Summe der Wahrscheinlichkeit über alle Ausgangszustände der Probe und der Summe über alle Wahrscheinlichkeiten für den Übergang von $\left|\mathbf{k}_{\mathrm{i}} n_{\mathrm{i}}\right\rangle$ nach $\left|\mathbf{k}_{\mathrm{f}} n_{\mathrm{f}}\right\rangle$

$$
W\left(\mathbf{k}_{\mathrm{i}} \rightarrow \mathbf{k}_{\mathrm{f}}\right)=\sum_{n_{\mathrm{i}}} p\left(n_{\mathrm{i}}\right) \sum_{n_{\mathrm{f}}} W\left(\mathbf{k}_{\mathrm{i}} n_{\mathrm{i}} \rightarrow \mathbf{k}_{\mathrm{f}} n_{\mathrm{f}}\right)
$$

unter Beachtung der Besetzungswahrscheinlichkeit

$$
p\left(n_{\mathrm{i}}\right)=\frac{\mathrm{e}^{E_{i} / k_{\mathrm{B}} T}}{\sum_{i} \mathrm{e}^{E_{i} / k_{\mathrm{B}} T}} .
$$

Mit der Annahme ebener Wellen für $|\mathbf{k}\rangle$ ist die Anzahl der in den Raumwinkel $\mathrm{d} \Omega$ und Frequenzintervall d $\omega$ gestreuten Neutronen, vgl. [Scherm 1972], der sogenannte doppelt differenzielle Streuquerschnitt,

$$
\begin{aligned}
\frac{\mathrm{d}^{2} \sigma}{\mathrm{d} \Omega \mathrm{d} \omega} & =\left(\frac{m_{\mathrm{n}}}{2 \pi \hbar^{2}}\right)^{2} \frac{k_{\mathrm{i}}}{k_{\mathrm{f}}} \sum_{n_{0}, n} p\left(n_{0}\right)\left|\left\langle\mathbf{k}_{\mathrm{f}} n_{\mathrm{f}}|V(\mathbf{r})| \mathbf{k}_{\mathrm{i}} n_{\mathrm{i}}\right\rangle\right|^{2} \delta\left(\frac{E_{\mathrm{i}}-E_{\mathrm{f}}}{\hbar}-\omega\right) \\
& =\frac{1}{N} \frac{k_{\mathrm{i}}}{k_{\mathrm{f}}} \sum_{n_{0}, n} p\left(n_{0}\right)\left|\sum_{i=1}^{N}\left\langle n_{\mathrm{f}}\left|b_{i} \mathrm{e}^{\imath \mathbf{Q R}}\right| n_{\mathrm{i}}\right\rangle\right|^{2} \delta\left(\frac{E_{\mathrm{i}}-E_{\mathrm{f}}}{\hbar}-\omega\right),
\end{aligned}
$$

mit $N$, der Anzahl der streuenden Atome; vgl. Abbildung 1.7. 


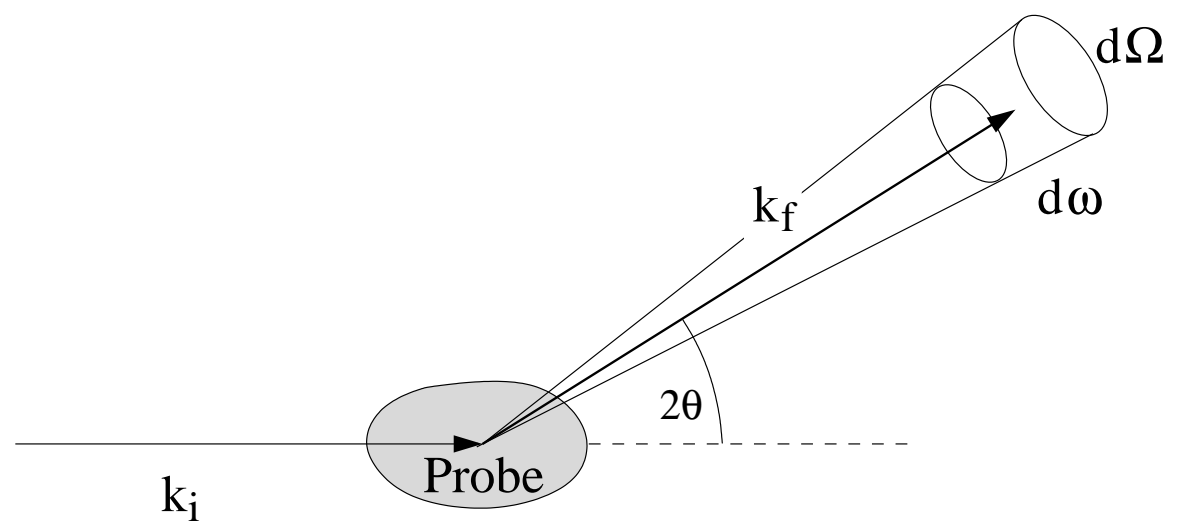

Abbildung 1.7: Anschauliche Darstellung des doppelt differnziellen Streuquerschnitts $\mathrm{d}^{2} \sigma / \mathrm{d} \Omega \mathrm{d} \omega$. Er gibt die Wahrscheinlichkeit an, daß ein Neutron mit dem Wellenvektor $\mathbf{k}_{\mathrm{i}}$ von der Probe in den Raumwinkel d $\Omega$ gestreut wird, und dabei sich die Energie des Neutrons um $\hbar \omega$ ändert.

Wird durch das einfallende Neutron in der Probe ein Phonon erzeugt, oder vom Phonon Energie auf das Neutron übertragen, gelten Energie- und Quasiimpulserhaltung aus Gleichung (1.21), so daß

$$
\mathbf{Q}=\mathbf{G}+\mathbf{q} \quad \text { und } \quad \Delta E=\hbar \omega_{\mathbf{q} j}
$$

sind, wenn q der Wellenvektor und $\omega_{\mathbf{q} j}$ die Kreisfrequenz des Phonons ist. Die Streufunktion für einen Streuprozeß an einem einzelnen Phonon ist [Dorner 1982]

$$
S_{j}(\mathbf{Q}, \omega)=\left|\sum_{i}^{N} b_{i} \frac{1}{\sqrt{m_{i}}} \mathbf{Q} \cdot \mathbf{e}_{j}(\mathbf{q}) \mathrm{e}^{-W(\mathbf{Q})+l \mathbf{Q} \mathbf{r}_{i}}\right|^{2} \cdot P_{j}\left(\omega, \omega_{\mathbf{q} j}, T\right)
$$

mit der Antwortfunktion $P_{j}\left(\omega, \omega_{\mathbf{q} j}, T\right)$. Gleichung (1.34) enthält eine Art „Meßvorschrift" für ein inelastischen Neutronenstreuexperiment an Phononen. Die Experimentanordnung sollte so gewählt sein, daß für longitudinale Phononen $\mathbf{Q}$ möglichst parallel zu q und für transversale Phononen $\mathbf{Q}$ möglichst senkrecht zu q ist. Für einen gedämpften harmonischen Oszillator kann für kleine Dämpfungen $\Gamma_{\mathbf{q} j}$ die Antwortfunktion

$$
P_{j}\left(\omega, \omega_{\mathbf{q} j}, T\right)=\frac{1}{\mathrm{e}^{\frac{\hbar \omega}{k_{\mathrm{B}} T}}-1} \cdot\left[\frac{\Gamma_{\mathbf{q} j}}{\left(\omega-\omega_{\mathbf{q} j}\right)^{2}+\Gamma_{\mathbf{q} j}^{2}}-\frac{\Gamma_{\mathbf{q} j}}{\left(\omega+\omega_{\mathbf{q} j}\right)^{2}+\Gamma_{\mathbf{q} j}^{2}}\right]
$$

angesetzt werden. Wird der Bose-Faktor entwickelt

$$
\frac{1}{\mathrm{e}^{\frac{\hbar \omega}{k_{\mathrm{B}} T}}-1} \approx \frac{k_{\mathrm{B}} T}{\hbar \omega}
$$




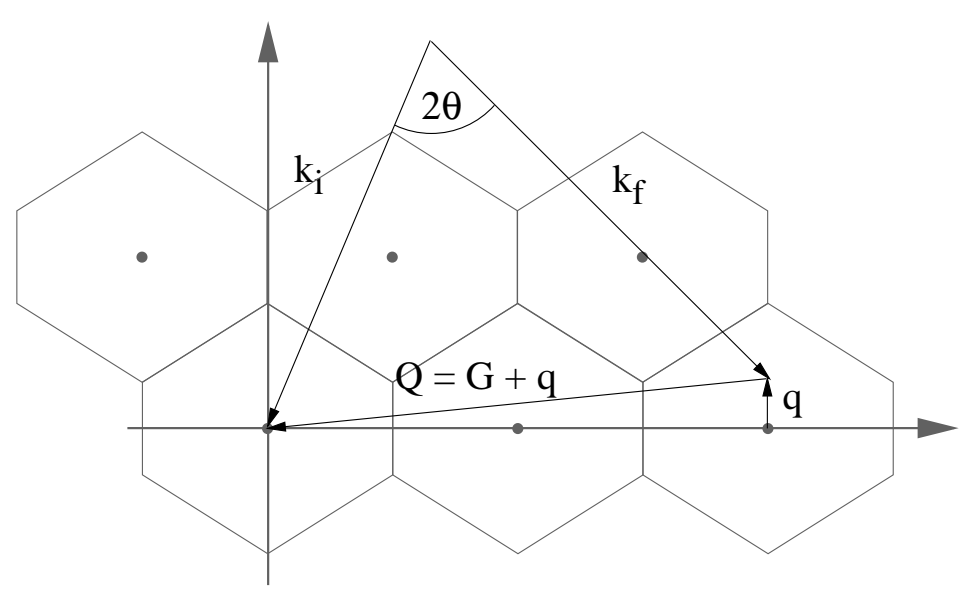

Abbildung 1.8: Das Streudreieck im elastischen Fall $\left(k_{\mathrm{i}} \neq k_{\mathrm{f}}\right)$

und der in Klammern stehende Term auf einen Nenner gebracht, lautet die Antwortfunktion

$$
P_{j}\left(\omega, \omega_{\mathbf{q} j}, T\right)=\frac{k_{\mathrm{B}} T}{\hbar} \cdot \frac{4 \omega_{\mathbf{q} j} \Gamma_{\mathbf{q} j}}{\left(\omega^{2}-\omega_{\mathbf{q} j}^{2}\right)^{2}+2 \Gamma^{2}\left(\omega^{2}+\omega_{\mathbf{q} j}^{2}\right)+\Gamma_{\mathbf{q} j}^{4}} .
$$

Beim gedämpften harmonischen Oszillator nach (1.37) unterscheiden sich die Frequenz mit maximaler Intensität $\omega_{\max }$ und die quasiharmonische Frequenz $\omega_{j}$ des harmonischen Oszillators um

$$
\omega_{\max }=\sqrt{\omega_{\mathbf{q} j}^{2}-\Gamma_{\mathbf{q} j}^{2}} .
$$

Je größer die Dämpfung, um so stärker wird das Intensitätsmaximum zu kleineren Frequenzen verschoben. In der harmonischen Näherung, in der die Dämpfung gegen Null geht, ist die Antwortfunktion des harmonischen Oszillators

$$
P_{j}\left(\omega, \omega_{\mathbf{q} j}, T\right)=\frac{\omega}{\mathrm{e}^{\frac{\hbar \omega}{k^{T}}}-1} \cdot \frac{\delta\left(\omega \pm \omega_{\mathbf{q} j}\right)}{\omega^{2}},
$$

mit $\omega+\omega_{\mathbf{q} j}$ für einen Energiegewinn bzw. $\omega-\omega_{\mathbf{q} j}$ für einen Energieverlust des Neutrons beim Streuproze ${ }^{9}$.

In Abbildung 1.8 ist das Streudreieck für einen inelastischen Fall abgebildet. Der Wellenvektor q des zu messenden Phonons ist annähernd senkrecht zu Q. Dies bedeutet, daß die Polarisation der Auslenkung senkrecht zur Ausbreitungsrichtung ist, und es sich bei dem Streudreieck um ein Streudreieck für eine Messung an einem transversalen Phonon handelt.

\footnotetext{
${ }^{9}$ Die Frequenz eines Phonons ist immer positiv, auch wenn der Energieübertrag vom Neutron auf die Probe positiv oder negativ sein kann.
} 
In der Neutronenstreuung werden Punkte und Vektoren im reziproken Gitter in Einheiten von reziproken Gittervektoren angegeben. Für die Natriumchloridstruktur sind dies vielfache von $2 \pi / a$. Dabei wird üblicherweise die Variable $\xi$ verwendet: Ein Punkt mit den Koordinaten $\left(\xi_{h}, \xi_{k}, \xi_{l}\right)$ liegt demnach in absoluten Einheiten bei $\left(\xi_{h} \cdot 2 \pi / a, \xi_{k}\right.$. $\left.2 \pi / b, \xi_{l} \cdot 2 \pi / c\right)$. Mit dieser Konvention kann z.B. Gleichung (1.17) für die Schallgeschwindigkeit eines TA Phonons in $\left[\begin{array}{lll}1 & 0 & 0\end{array}\right]$ als

$$
v=\lim _{q \rightarrow 0}\left(\frac{\partial \omega}{\partial q}\right)=\lim _{\xi \rightarrow 0}\left(\frac{2 \pi}{2 \pi / a} \frac{\partial v}{\partial \xi}\right)=a \cdot \frac{\partial v}{\partial \xi}
$$

geschrieben werden. 


\section{Das Silber-Natriumchlorid System}

Silberchlorid (Hornsilber, Chlorargyrit) [Gmelin 1972, Falbe und Gefitz 1992, Hollemann und Wiberg 1985] ist lichtempfindlich und im reinen Zustand ein weißes Pulver mit einem Schmelzpunkt von $455{ }^{\circ} \mathrm{C}$ und einem Siedepunkt von $1554{ }^{\circ} \mathrm{C}$. Es hat eine Dichte von $5.589 \mathrm{~g} / \mathrm{cm}^{3}$ und eine Molmasse von $143.32 \mathrm{~g} / \mathrm{mol}$. Es kristallisiert in der Natriumchloridstruktur mit einer Gitterkonstanten von $a=5.539 \AA$ bei Raumtemperatur [Caspary 1998] und hat einen linearen thermischen Ausdehnungskoeffizienten von $\alpha \approx 35 \cdot 10^{-6} 1 / \mathrm{K}$ [Jantzen und Herman 1978]. Bei $25^{\circ} \mathrm{C}$ hat es ein Löslichkeitsprodukt von $K_{\mathrm{L}}=1.7 \cdot 10^{-10}$ entsprechend $1.3 \cdot 10^{-5}$ mol $/ 1$ oder $1.8 \mathrm{mg} / 1$. Natriumchlorid (Kochsalz, Steinsalz) [Falbe und Gefitz 1991] ist eine als Pulver weiße und als Einkristall farblose Substanz. Der Schmelzpunkt liegt bei $801^{\circ} \mathrm{C}$, der Siedepunkt bei $1440{ }^{\circ} \mathrm{C}$. Es hat eine Dichte von $2.164 \mathrm{~g} / \mathrm{cm}^{3}$, und die Molmasse beträgt $58.443 \mathrm{~g} / \mathrm{mol}$. Es hat die gleiche Kristallstruktur wie das Silberchlorid mit einer Gitterkonstanten bei Raumtemperatur von $a=5.633 \AA$ [Caspary 1998] und einen linearen thermischen Ausdehnungskoeffizienten von $\alpha \approx 44 \cdot 10^{-6} 1 / \mathrm{K}$ [Jantzen und Herman 1978].

Die Kinetik der Entmischungsreaktion im Mischkristallsystem $\mathrm{Ag}_{x} \mathrm{Na} a_{1-x} \mathrm{Cl}$ war in der Vergangenheit kaum Gegenstand des wissenschaftlichen Interesses. Es wurden hauptsächlich thermodynamische Aspekte des Mischkristallsystems untersucht. Der Grund hierfür ist das nahezu ideale Verhalten als binäre Mischung [Schimschal-Thölke u. a. 1995]. Das Phasendiagramm im Subsolidusbereich ist in Abbildung 2.1 gezeigt. Es wurde 1920 von Zemczuzny bestimmt und von [Stokes und Li 1962] und [Sinistri u. a. 1971] nachgemessen. Nach Sinistri ist die obere kritische Zusammensetzung $x_{\text {krit }}=0.513$ und die kritische kritische Temperatur $T_{\text {krit }}=198{ }^{\circ} \mathrm{C}$. Die von dieser Arbeitsgruppe veröffentlichte Mischungslücke läßt sich sehr gut durch eine nach unten geöffnete Parabel beschreiben, die nach der Zusammensetzung aufgelöst

$$
x_{1,2}=\frac{1.034}{2} \pm \sqrt{\frac{1.034^{2}}{4}+\frac{28.59^{\circ} \mathrm{C}-T}{627.5^{\circ} \mathrm{C}}}
$$

ergibt. Strukturell liegt das Mischkristallsystem wie die reinen Komponenten in der $\mathrm{NaCl}$-Struktur vor, und es gilt für die Gitterkonstante die Regel von Vegard. Die Gitterkonstante eines Mischkristallsystems ergibt sich aus der Linearkombination der Gitterkonstanten der reinen Substanzen $a_{1}$ und $a_{2}$

$$
a_{\text {mix }}=x_{0} \cdot a_{1}+\left(1-x_{0}\right) \cdot a_{2}
$$




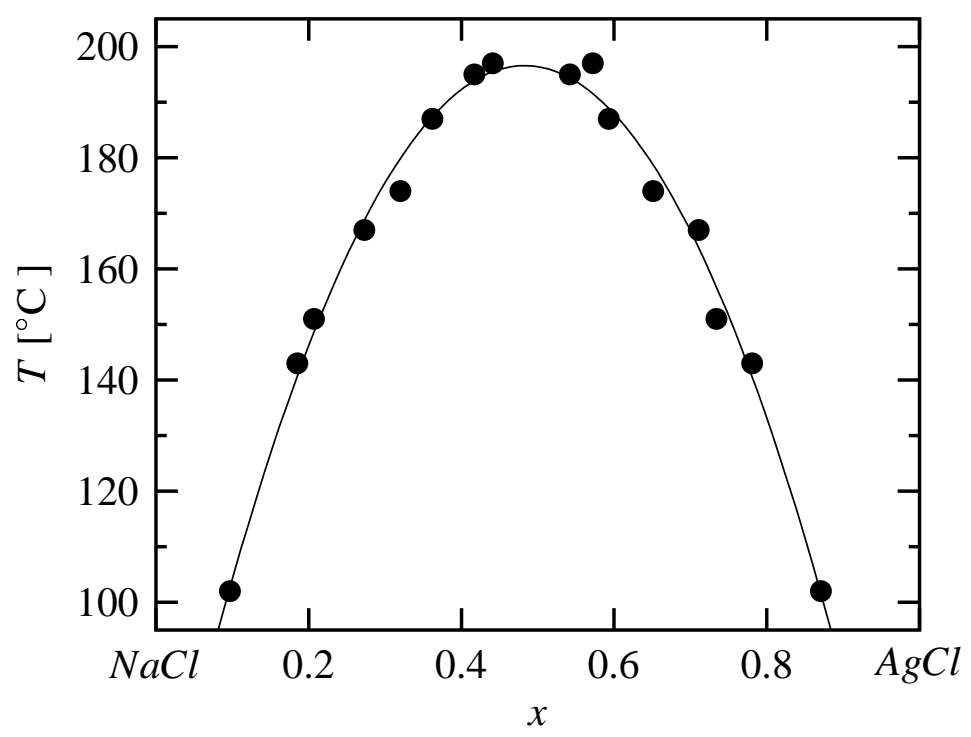

Abbildung 2.1: Die Mischungslücke von $A g_{x} N a_{1-x} C l$ nach [Sinistri u. a. 1971]

wenn die beiden Komponenten und der Mischkristall die gleiche Kristallstruktur besitzen. Am Phasendiagramm fällt lediglich auf, daß es keinen kongruenten Schmelzpunkt gibt, vgl. Abbildung 3.2, der sonst typisch für Systeme mit einer Mischungslücke ist.

Eine erste Untersuchung an entmischendem Silber-Natriumchlorid wurde von [Hendricks u. a. 1964] durchgeführt. Es wurde eine Schmelze mit $x_{0}=0.21^{1}$ in Eiswasser abgeschreckt und aus dem Schmelzkuchen eine Pulverprobe präpariert. Es folgte die Untersuchung der zeitlichen Entwicklung von Ausscheidungsgrößen bei Raumtemperatur anhand von Röntgenkleinwinkelstreuung. Eigene Versuche ergaben, daß es deutliche Unterschiede in den Röntgendiffraktogrammen im Bereich $2 \theta \approx 30-60^{\circ}$ gibt, je nach dem, ob die Probe nach dem Auslagern gemörsert wurde, oder ob eine gemörserte Probe homogenisiert und dann ausgelagert wurde. Erstgenannte zeigt eine deutliche größere Reflexaufspaltung. Die Relaxation des Gitters ist jedoch - wie gezeigt werden wird - ein charakteristisches Merkmal für eine entmischte Probe. Die Probenpräparation hat folglich einen Einfluß auf die Entmischung, und die Ergebnisse in [Hendricks u. a. 1964] müssen kritisch bewertet werden.

Mit der Untersuchung der Entmischungskinetik des analogen $\mathrm{Ag}{ }_{x} N a_{1-x} \mathrm{Br}$-Systems [Trzeciok 1976, Eckold 1991, Windgasse 1994, Windgasse u. a. 1997, Eckold 2001] wurde das Silber-Natriumchloridsystem diesbezüglich wieder Gegenstand des wissenschaftlichen Interesses. Silber-Natriumchlorid unterscheidet sich vom analogen Bro-

\footnotetext{
${ }^{1}$ Der Stoffmengenanteil bezieht sich in dieser Arbeit auf den Silberchloridanteil: $x=\frac{n_{A g C l}}{n_{A g C l}+n_{N a C l}}$. Der Index 0 wird i.d.R. für die mittlere Zusammensetzung $\left(x_{0}\right)$ verwendet und die Indizes 1 und 2 entsprechend für die natrium- $\left(x_{1}\right)$ und silberreiche $\left(x_{2}\right)$ Phase.
} 
midsystem strukturell insbesondere in der Differenz der Gitterkonstanten der reinen Komponenten. Liegt die Differenz im Bromidsystem bei $3.4 \%$, ist sie im Chloridsystem nur $1.7 \%$. Analoge Untersuchungen in beiden Systemen sollten Informationen über den Einfluß der Struktur auf den Entmischungsprozeß liefern.

Die ersten eingehenden Untersuchungen zur Entmischungskinetik in Silber-Natriumchlorid wurden mit zeitaufgelöster Neutronendiffraktion und Neutronenkleinwinkelstreuung ${ }^{2}$ (SANS) an Pulverproben durchgeführt [Caspary 1998, Caspary u. a. 2001]. Bei einer Entmischung von Silber-Natriumchlorid sollte die Gitterkonstante der homogenen Phase in die Gitterkonstanten der entmischten Phasen übergehen. Die Neutronendiffraktionsexperimente an Pulverproben mit $x_{0}=0.5$ zeigten jedoch bei Temperaturen im Bereich von $50-198{ }^{\circ} \mathrm{C}$ auf einer Zeitskala von mehr als $10^{5} \mathrm{~s}$ keine Aufspaltung des Braggreflexes. Nach mehreren Stunden lag ein breiter Braggreflex, also eine Gitterkonstantenverteilung, vor. In einem intermediären Zeitbereich in der Größenordnung von $10^{3} \mathrm{~s}$ änderte sich die Form des Braggreflexes nicht. Die dazugehörige Gitterkonstante entsprach der Gitterkonstante der homogenen Phase. Dies entspricht dem Konzept einer theoretischen homogenen Phase im Zweiphasengebiet, wie sie auf Seite 8 beschrieben wurde. Darüber hinaus fand sich eine erhöhte Intensität im Abstand $\Delta Q= \pm 0.02 \AA^{-1}$ vom Zentrum des Braggreflexes. Diese Reflexpositionen liegen außerhalb des $Q$-Bereiches, der durch die Reflexpositionen der reinen Komponenten begrenzt wird.

Die Neutronenkleinwinkelstreuung erlaubt es, Strukturen im mesoskopischen Bereich aufzulösen. Wie der Name bereits sagt, wird bei SANS-Experimenten bei kleinen Streuwinkeln gemessen. Die untersuchten räumlichen Strukturen in der Probe sind dann so groß, daß nicht mehr wie in Gleichung (1.24) über alle Streuzentren summiert werden muß. Die Streulänge kann durch eine mittlere Streulängendichte $\rho(\mathbf{r})=\Sigma b / v$ ersetzt werden. Die zu messende Intensität ist dann

$$
|F(\mathbf{Q})|^{2}=\left|\int_{V} \rho(\mathbf{r}) e^{l \mathbf{Q r}} \mathrm{d} V\right|^{2} .
$$

Die Neutronenkleinwinkelstreuung liefert dabei Informationen über die Morphologie und den Entmischungsgrad während der Entmischung.

Die spinodale Entmischung führt zu periodischen Konzentrationsprofilen mit einem charakteristischen dominierenden Wellenvektor $Q_{\max }=2 \pi / \lambda$. In allen SANS-Diffraktogrammen bei Temperaturen unterhalb von $174 \pm 3{ }^{\circ} \mathrm{C}$ fand sich genau ein solches Korrelationsmaximum $Q_{\max }$. Der obere Entmischungspunkt ist folglich mit $174{ }^{\circ} \mathrm{C}$ anzugeben. Die Unterdrückung der Spinodalen von $24 \pm 3{ }^{\circ} \mathrm{C}$ stimmt dabei gut mit der berechneten Unterdrückung von $21^{\circ} \mathrm{C}$ überein.

\footnotetext{
${ }^{2}$ Zur Neutronenkleinwinkelstreuung siehe z.B. [Guinier und Fournet 1955, Gerold 1967, Gerold 1978,
} Glatter und Kratky 1982,Feigin und Svergun 1987, Windsor 1988, Brunner-Popela und Glatter 1997]. 


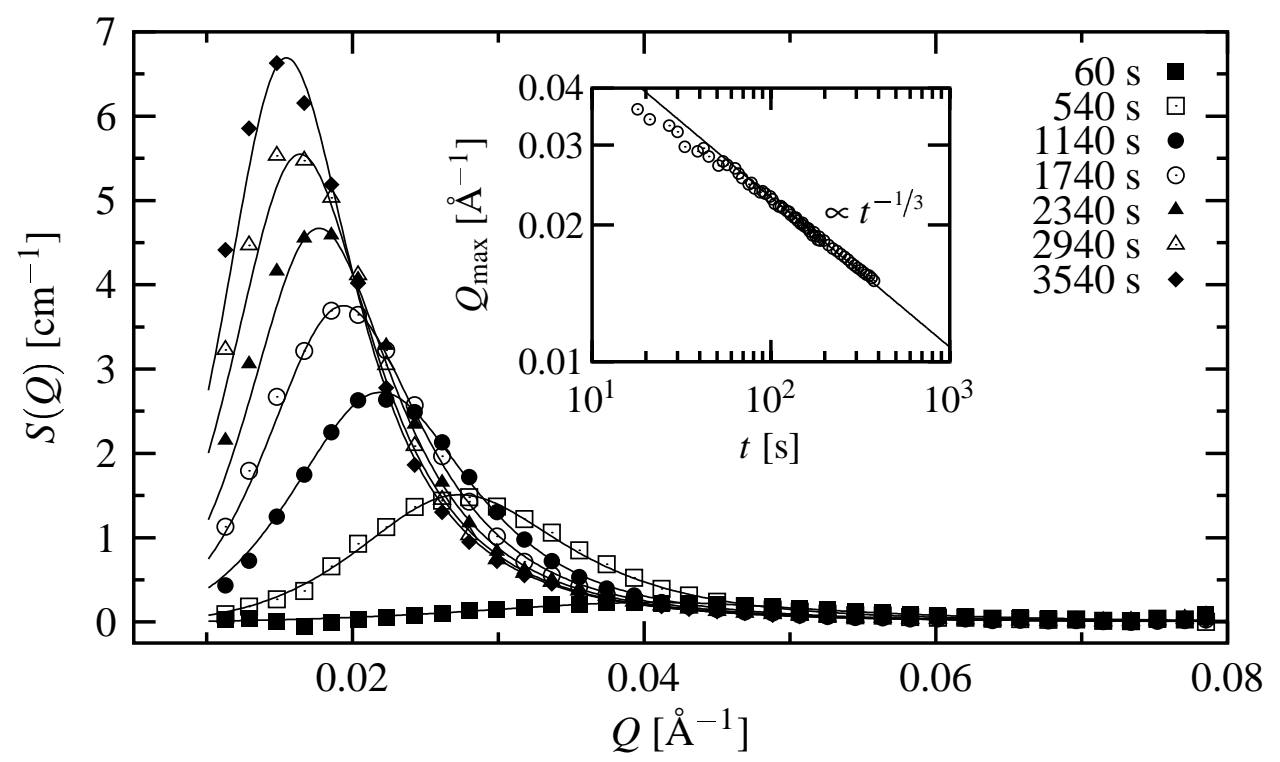

Abbildung 2.2: Die zeitliche Entwicklung der SANS-Intensität bei $100{ }^{\circ} \mathrm{C}$ und die Lage des Korrelationsmaximums $Q_{\max }$ in Abhängigkeit von der Zeit

In Abbildung 2.2 ist die zeitliche Entwicklung der SANS-Intensität bei $100{ }^{\circ} \mathrm{C}$ gezeigt, die charakteristisch für alle Auslagerungstemperaturen ist. Alle S ANS-Diffraktogramme können quantitativ mit dem phänomenologischen Ansatz von [Furukawa 1984]

$$
S(Q)=S\left(Q_{\max }\right) \cdot\left(1+\frac{\gamma}{\beta}\right) \frac{\left(\frac{Q}{Q_{\max }}\right)^{\beta}}{\frac{\gamma}{\beta}+\left(\frac{Q}{Q_{\max }}\right)^{\beta+\gamma}}
$$

beschrieben werden. Die beiden Parameter $\beta$ und $\gamma$ charakterisieren den Verlauf der Funktion für $Q \rightarrow 0$ und $Q \rightarrow \infty$. Die durchgezogenen Linien in Abbildung 2.2 sind die jeweils angepaßte Furukawa-Funktion. Aus der Anpassung der Furukawafunktion werden die chrakteristischen Größen der Diffraktogramme erhalten. Es werden z.B. nach 1800 s Korrelationswellenlängen von $330 \AA\left(100{ }^{\circ} \mathrm{C}\right), 420 \AA\left(120{ }^{\circ} \mathrm{C}\right)$ und $600 \AA$ $\left(160^{\circ} \mathrm{C}\right)$ erreicht.

In den frühen Stadien ist nach Cahn ein Korrelationsmaximum bei konstantem $Q_{\max }$ zu erwarten. Das Korrelationsmaximum verschiebt sich jedoch kontinuierlich zu kleineren Wellenvektorüberträgen. Diese Abweichung folgt daraus, daß bereits in diesen Entmischungsstadien die lineare Näherung für die freie Enthalpiedichte nicht mehr gültig ist. Stattdessen führen nichtlinearen Terme zu einer Verschiebung von $Q_{\max }$. Bei einer höheren Zeitauflösung bei $120{ }^{\circ} \mathrm{C}$ fand sich bei frühen Zeiten $t<10^{2}$ s eine mit $t \rightarrow 0$ abnehmende Verschiebung von $Q_{\max }$. Im analogen $\mathrm{AgBr}-\mathrm{NaBr}$-System konnte dieser Bereich mit konstantem Korrelationsmaximum zeitlich aufgelöst werden. Die Verschiebung von $Q_{\max } \propto t^{-1 / 3}$ zu späteren Zeiten ist nach [Lifshitz und Slyozov 1961] 


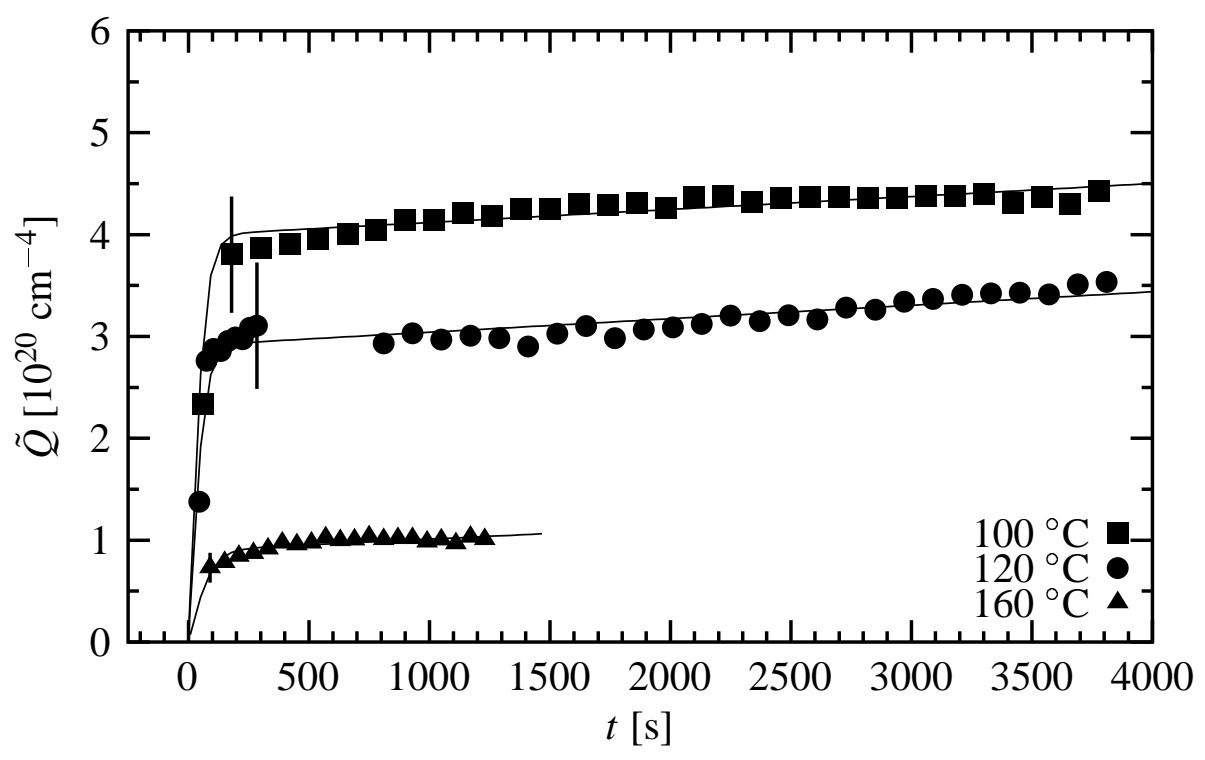

Abbildung 2.3: Die zeitliche Entwicklung der Integralen Intensität

das typische Verhalten für einen diffusionskontrollierten Vergröberungsprozeß.

Mit Hilfe der Integralen Intensität $\tilde{Q}$ kann die Kinetik der Entmischung verfolgt werden. Diese ergibt sich für ein binäres System mit scharfer Phasentrennung zwischen den Ausscheidungen mit der Teilchenzahldichte $\Omega_{i}$, der Streulängendichte $\rho_{i}$ und dem Entmischungsgrad $\alpha \mathrm{zu}$

$$
\begin{aligned}
\tilde{Q} & =4 \pi \frac{\mathrm{d} \Sigma}{\mathrm{d} \Omega}(Q) Q^{2} \mathrm{~d} Q \\
& =8 \pi^{3}\left(\frac{\Omega_{1} x_{1}-\Omega_{0} x_{0}}{\Omega_{1} x_{1}-\Omega_{2} x_{2}} \rho_{2}^{2}+\frac{\Omega_{0} x_{0}-\Omega_{2} x_{2}}{\Omega_{1} x_{1}-\Omega_{2} x_{2}} \rho_{1}^{2}-\rho_{0}\right) \alpha .
\end{aligned}
$$

Im Falle gleicher Teilchenzahldichten vereinfacht sich Gleichung (2.5) zur bekannten Beziehung [Gerold 1961]

$$
\tilde{Q}=8 \pi^{3}\left(\rho_{01}-\rho_{02}\right)^{2} \cdot\left(x_{0}-x_{1}\right)\left(x_{2}-x_{0}\right) \cdot \alpha
$$

mit den Streulängendichten $\rho_{01}$ und $\rho_{02}$ der beiden reinen Komponenten. Abbildung 2.3 zeigt die Entwicklung der Integralen Intensität für drei verschiedene Temperaturen. Innerhalb weniger $10^{2} \mathrm{~s}$ steigt $\tilde{Q}$ sprunghaft an und nimmt dann nur noch sehr langsam zu. Dabei ist die Zeitspanne, die dafür benötigt wird, nahezu unabhängig von der Temperatur. Die erreichte Integrale Intensität entspricht jedoch nur einem Bruchteil dessen, was für einen völlig entmischten Zustand berechnet werden kann: $\tilde{Q}_{\max }\left(100^{\circ} \mathrm{C}\right)=18.5$. $10^{20} \mathrm{~cm}^{-4}, \tilde{Q}_{\max }\left(120^{\circ} \mathrm{C}\right)=14.7 \cdot 10^{20} \mathrm{~cm}^{-4}$ und $\tilde{Q}_{\max }\left(160{ }^{\circ} \mathrm{C}\right)=7.0 \cdot 10^{20} \mathrm{~cm}^{-4}$. Aus den experimentell bestimmten Integralen Intensitäten können mit Gleichung (2.6) und 
der Annahme einer symmetrischen Mischungslücke die Konzentrationen $x_{1}^{\prime}$ und $x_{2}^{\prime}$ berechnet werden. Die Ergebnisse sind in Abbildung 2.4 aufgetragen. Die mit den SANSExperimente nachgewiesene Entmischung erfolgt in einem starren Gitter, wie die Neutronendiffraktionsexperimente ergaben. Bei der aus der Integralen Intensität bestimmten Kurve $T$ vs. $x$ in Abbildung 2.4 handelt es sich somit um die kohärente Spinodale. Die berechneten Zusammensetzungen entsprechen $x_{1}^{\prime}$ und $x_{2}^{\prime}$ aus Abbildung 1.1. Der obere Entmischungspunkt bei $x=0.5$ und $173{ }^{\circ} \mathrm{C}$ ist demzufolge der obere kritische Entmischungspunkt.

Nach den Vorarbeiten [Caspary 1998, Caspary u. a. 2001] ergibt sich zusammenfassend folgendes Bild für die Entmischung: Nach dem Abschrecken entmischt Silber-Natriumchlorid nahezu temperaturunabhängig innerhalb weniger hundert Sekunden. Es werden dabei die Konzentrationen der kohärente Binodale $x_{1}^{\prime}$ und $x_{2}^{\prime}$ erreicht. Während dieser schnellen Entmischung bleibt die Gitterkonstante der homogenen Phase erhalten. An diese schnelle Entmischung schließt sich eine wesentlich langsamere Entmischung an. Die breite Gitterkonstantenverteilung nach Stunden und der langsame Anstieg der Integralen Intensität läßt einen Zusammenhang zwischen der chemischen Entmischung und der Gitterrelaxation schließen. Die in den Neutronendiffraktionsexperimenten gefundenen Intensität, die keiner Mischphase zugeordnet werden konnte, legt im Vergleich mit den SANS-Ergebnissen die Vermutung von Satelliten nahe, die aber aufgrund der ungenügenden Statistik in den Diffraktionsexperimenten nicht verifiziert werden konnte.

Um den vermuteten spinodalen Entmischungsmechanismus nachweisen zu können, muß die Zeitauflösung und die Längenskala, auf denen die Entmischung untersucht wird, verfeinert werden. Der kleinste mögliche Abstand ist der Abstand zwischen $\mathrm{Ag}^{+}$ und $\mathrm{Ag}^{+}$bzw. $\mathrm{Na}^{+}$, also eine Gitterkonstante. Um eine Entmischung beschreiben zu können, muß aber das untersuchte Volumen hinreichend groß sein. Das Volumen einer Elementarzelle wäre für eine kontinuierliche Beschreibung zu klein. In ihr gibt es nur vier Zusammensetzungen $x=0,0.25,0.5,0.75$ und 1. Bei einem Volumen aus $3 \cdot 3 \cdot 3$ Elementarzellen, das entspricht einer Kantenlänge von ca. $17 \AA$, gibt es schon mehr als 100 mögliche Zusammensetzungen. Dies liegt in der räumlichen Größenordnung, in der interatomare Wechselwirkungen sehr gut an Phononen untersucht werden können. Die mittlere freie Weglänge $\ell$ eines akustischen Phonons kann aus der Wärmeleitung berechnet werden [Kittel 1996]. In Natriumchlorid mit der Wärmeleitzahl $K=0.07 \mathrm{~W} / \mathrm{cm} \mathrm{K}$, der spezifischen Wärmekapazität pro Volumen $C=1.88 \mathrm{~J} / \mathrm{cm}^{3} \mathrm{~K}$ und der Schallgeschwindigkeit $v=2 \cdot 10^{5} \mathrm{~cm} / \mathrm{s}$ ist die mittlere freie Weglänge

$$
\ell=3 \frac{K}{C v} \approx 50 \AA \text {. }
$$

Experimentell sind die Phononen durch inelastische Neutronenstreuung zugänglich. Dabei wechselwirkt das Neutron nur lokal mit der Probe, selbst wenn das Phonon 


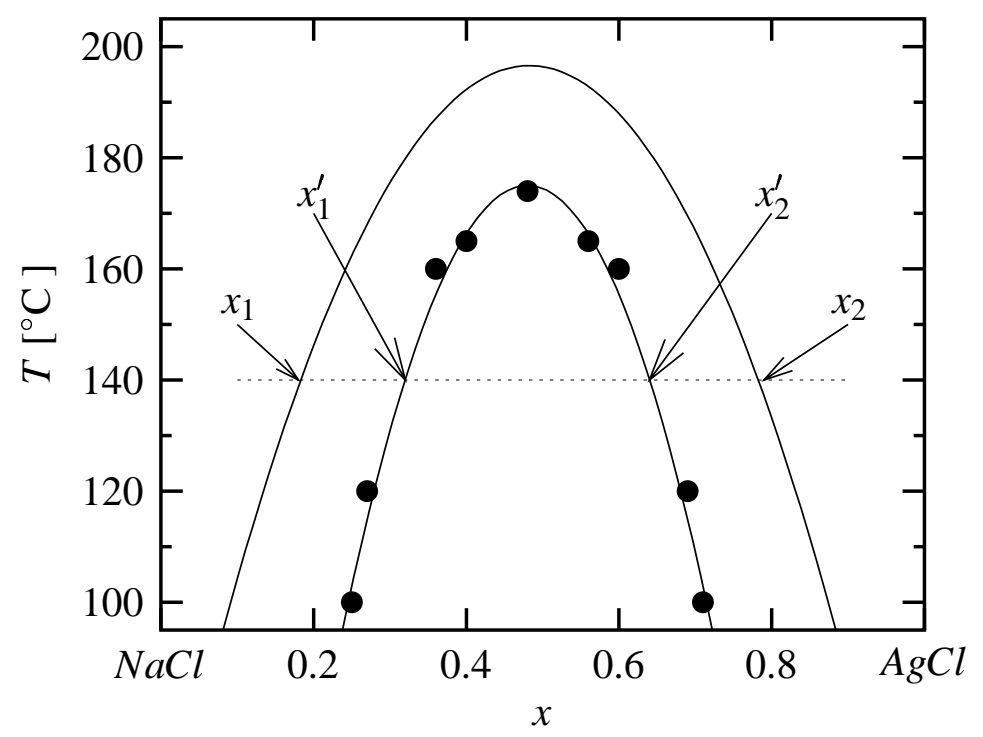

Abbildung 2.4: Die Binodale und die kohärente Spinodale von $\mathrm{Ag}_{x} N a_{1-x} \mathrm{Cl}$ wie sie mittels SANs [Caspary u. a. 2001] bestimmt wurden. Es sind beispielhaft für $140{ }^{\circ} \mathrm{C}$ die Zusammensetzungen für die natrium- und silberreiche Binodalkonzentration $x_{1}$ und $x_{2}$ und die Konzentrationen $x_{1}^{\prime}$ und $x_{2}^{\prime}$ der kohärenten Binodale mit Pfeilen markiert.

eine größere Laufweite hat. Zeitaufgelöste Messungen von Phononenspektren während der Entmischung liefern demzufolge detaillierte Informationen über die Entmischung auf mikroskopischer Skala, weil sie die lokale Zusammensetzung widerspiegeln. Während Phononen in homogenen Mischkristallen schon untersucht wurden, z.B. [Aslam u. a. 1976, Perry u. a. 1978, Grünewald und Scharnberg 1978, Goyal und Asthana 1991b, Goyal und Asthana 1991a], sind dem Autor nur Untersuchungen zum Einfluß der Entmischung auf Phononen oder Phononendispersionen in entmischten Systemen bekannt, an denen er beteiligt ist.

Abbildung 2.5 zeigt die Dispersion für die akustischen Moden in reinem Silber- und Natriumchlorid entlang [ $\left.\begin{array}{lll}1 & 0 & 0\end{array}\right]$ - und [ $\left[\begin{array}{lll}0 & 1 & 1\end{array}\right]$, und die dazugehörigen Brillouin-Zonen in der $\left[\begin{array}{lll}0 & 0 & 1\end{array}\right]$ - und $\left[\begin{array}{lll}0 & 1 & \overline{1}\end{array}\right]$-Streuebene ${ }^{3}$. Das jeweilige transversal akustische Phononen mit der Polarisation parallel zu [ $\left[\begin{array}{lll}0 & 1 & \overline{1}\end{array}\right]$ ist nicht mit aufgetragen. Das TA Phonon in $\left[\begin{array}{lll}1 & 0 & 0\end{array}\right]$ ist zweifach entartet. Wird dem Dispersionszweig des TA Phonons in der $\left[\begin{array}{lll}0 & 1 & 1\end{array}\right]$-Streuebene vom Brillouinzonenzentrum $\left(\begin{array}{llll}0 & 0 & 0\end{array}\right)$ entlang $\left[\begin{array}{lll}1 & 0 & 0\end{array}\right]$ gefolgt, wird bei X der Zonenrand erreicht. Dieser Punkt kann aber gleichermaßen vom benachbarten Zonenzentrum (lllll) erreicht werden, indem entlang [ $\left.\begin{array}{lll}0 & 1 & 1\end{array}\right]$ in Richtung Zonenrand gegangen wird. Das von ( $\left.\begin{array}{lll}0 & 0 & 0\end{array}\right)$ aus betrachtete TA Phonon entspricht von (lllll) aus gesehen einem longitudinal akustischen Phonon. Analog findet ein Übergang des LA Phonons

\footnotetext{
${ }^{3}$ Die ganze Brillouin-Zone für ein kubisches flächenzentriertes Gitter ist im Anhang .3 abgebildet.
} 

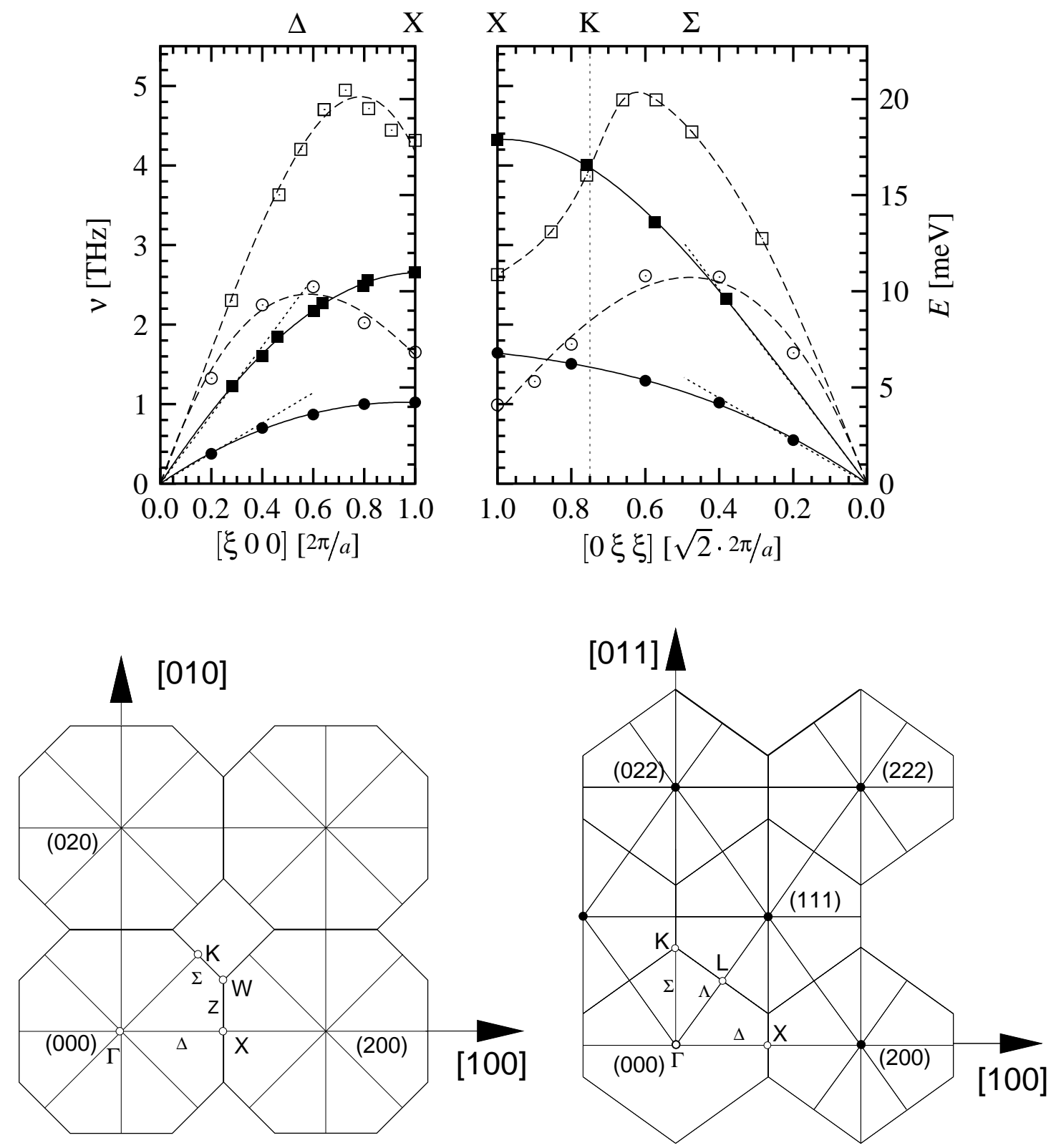

Abbildung 2.5: Oben: Die Dispersionskurven nach [Bilz und Kress 1979] der akustischen Moden von reinem $\mathrm{AgCl}(\bullet, \odot)$ und $\mathrm{NaCl}(\boldsymbol{\square}, \odot)$ in $\left[\begin{array}{lll}1 & 0 & 0\end{array}\right]$ - und [0 1011$]$-Richtung. Die gefüllten Symbole sind die transversal akustischen Zweige und die offenen Symbole die longitudinal akustischen Zweige. Die gepunkteten Linien in der Nähe des $\Gamma$-Punktes sind die aus den elastischen Konstanten extrapolierten Dispersion; siehe auch Tabelle 2.1. Unten: Die Brillouin-Zonen in der $\left[\begin{array}{lll}0 & 0 & 1\end{array}\right]$ - und der $\left[\begin{array}{lll}0 & 1 & 1\end{array}\right]$-Streuebene. 
von $\left[\begin{array}{lll}1 & 0 & 0\end{array}\right]$ in ein TA Phonon in $\left[\begin{array}{lll}0 & 1 & 1\end{array}\right]$ statt.

Mit Gleichung (1.18) und (1.40) kann die Schallgeschwindigkeit, die der Steigung der akustischen Dispersionszweige für $\xi \rightarrow 0$ äquivalent ist, berechnet werden. Für reines $\mathrm{AgCl}$ bzw. $\mathrm{NaCl}$ ergeben sich z. B. für die Schallgeschwindigkeiten bei Raumtemperatur nach Tabelle 2.1

$$
v_{\mathrm{AgCl}}^{\mathrm{TA}[100]}=\sqrt{\frac{6.22 \cdot 10^{9} \mathrm{~Pa}}{5589 \mathrm{~kg} / \mathrm{m}^{3}}}=1055 \mathrm{~m} / \mathrm{s} \quad \text { und } \quad v_{\mathrm{NaCl}}^{\mathrm{TA}[100]}=\sqrt{\frac{12.8 \cdot 10^{9} \mathrm{~Pa}}{2164 \mathrm{~kg} / \mathrm{m}^{3}}}=2432 \mathrm{~m} / \mathrm{s} .
$$

Mit Kenntnis der Temperatur bzw. Druckkoeffizienten

$$
T_{c_{i j}}=\frac{1}{c_{i j}} \cdot\left(\frac{\partial c_{i j}}{\partial T}\right)_{p} \quad \text { bzw. } \quad p_{c_{i j}}=\frac{1}{c_{i j}} \cdot\left(\frac{\partial c_{i j}}{\partial p}\right)_{T}
$$

ist es möglich, die Schallgeschwindigkeit für anderen Temperaturen oder Drücke zu extrapolieren, wenn die entsprechende Änderungen der Gitterkonstanten

$$
\alpha=\frac{1}{a}\left(\frac{\partial a}{\partial T}\right)_{p} \quad \text { bzw. } \quad \varkappa=\frac{1}{a} \cdot\left(\frac{\partial a}{\partial p}\right)_{T}
$$

bekannt sind. Ist z. B. $\Delta v / \Delta \xi$ die Steigung der linearisierten Dispersionskurve für $\xi \rightarrow 0$ bei der Temperatur $T$, ist

$$
\begin{aligned}
\left(\frac{\Delta v}{\Delta \xi}\right)_{T+\Delta T} & \approx \frac{1}{a(1+\alpha \Delta T)} \sqrt{\frac{c_{44}\left(1+T_{c_{44}} \Delta T\right)}{\frac{m}{[a(1+\alpha \Delta T)]^{3}}}} \\
& =\left(\frac{\Delta v}{\Delta \xi}\right)_{T} \cdot \sqrt{\left(1+T_{c_{44}} \Delta T\right) \cdot(1+\alpha \Delta T)}
\end{aligned}
$$

die Steigung bei $T+\Delta T$, und die Frequenz kann mit $v=\xi \cdot(\Delta v / \Delta \xi)_{T+\Delta T}$ berechnet werden.

Der Verlauf der Phononendispersionen kann für beide Substanzen mit Hilfe des Schalenmodells beschrieben werden [Schmunk und Winder 1970, Vijayaraghaven u. a. 1970, Fischer u. a. 1972]. Hierzu wurden die Parameter des Schalenmodells unter Berücksichtigung der elastischen Konstanten an die experimentell bestimmten Dispersionskurven angepaßt. Es fällt an den Ergebnissen auf, daß die Anpassungen für die Polarisierbarkeit des Kations in beiden Fällen negative Werte lieferten. Für das Natriumchlorid fand sich aber ein Parametersatz für das Schalenmodell mit einer positiven Polarisierbarkeit für das $\mathrm{Na}$-Ion, der mit Hilfe des Programmpaketes UNISOFT [Eckold 1992, Elter und Eckold 2000] erhalten wurde. Mit dem gleichen Programmpaket wurde im Rahmen dieser Arbeit versucht, einen Parametersatz für das Silberchlorid mit positiver Polarisierbarkeit zu finden, indem die Parameter an die Dispersion iterativ, 
Tabelle 2.1: Die elastischen Konstanten von $\mathrm{AgCl}$ und $\mathrm{NaCl}$ bei Raumtemperatur und deren Temperatur- und Druckabhängigkeit nach [Every und McCurdy 1992] und charakteristische Größen der Anfangssteigungen der Dispersionszweige

\begin{tabular}{|c|c|c|c|}
\hline \multicolumn{4}{|c|}{ Silberchlorid $\left(\rho=5589 \mathrm{~kg} / \mathrm{m}^{3}\right.$ und $\left.a=5.539 \AA\right)$} \\
\hline elast. Konstanten [GPa] & $\begin{array}{r}c_{11} \\
59.6\end{array}$ & $\begin{array}{r}c_{12} \\
36.1\end{array}$ & $\begin{array}{r}c_{44} \\
6.22\end{array}$ \\
\hline Temperaturkoeffizient $\left[10^{-4} 1 / \mathrm{K}\right]$ & -10.1 & -3.8 & -4.28 \\
\hline Druckkoeffizient $\left[10^{-12} 1 / \mathrm{Pa}\right]$ & 183 & 121 & -81.2 \\
\hline & $\mathrm{TA}\left[\begin{array}{lll}1 & 0 & 0\end{array}\right]$ & TA $\left[\begin{array}{lll}1 & 1 & 0\end{array}\right]$ & TA $\left[\begin{array}{lll}1 & 1 & 1\end{array}\right]$ \\
\hline Kombination der elast. Konst. [GPa] & $\begin{array}{r}c_{44} \\
6.22\end{array}$ & $\begin{array}{r}c_{44} \\
6.22\end{array}$ & $\begin{array}{r}c_{11}-c_{12}+c_{44} \\
29.7\end{array}$ \\
\hline$v_{\text {Schall }}[\mathrm{m} / \mathrm{s}]$ & 1055 & 1055 & 1331 \\
\hline$(\partial v / \partial \xi)[\mathrm{THz}]$ & 1.91 & 2.69 & 4.16 \\
\hline & $\mathrm{LA}\left[\begin{array}{lll}1 & 0 & 0\end{array}\right]$ & $\mathrm{LA}\left[\begin{array}{lll}1 & 1 & 0\end{array}\right]$ & $\mathrm{LA}\left[\begin{array}{lll}1 & 1 & 1\end{array}\right]$ \\
\hline Kombination der elast. Konst. [GPa] & $\begin{array}{r}c_{11} \\
59.6\end{array}$ & $\begin{array}{r}c_{11}+c_{12}+2 c_{44} \\
108\end{array}$ & $\begin{array}{r}c_{11}+2 c_{12}+4 c_{44} \\
157\end{array}$ \\
\hline$v_{\text {Schall }}[\mathrm{m} / \mathrm{s}]$ & 3266 & 3110 & 3057 \\
\hline$(\partial v / \partial \xi)[\mathrm{THz}]$ & 5.90 & 7.94 & 9.56 \\
\hline \multicolumn{4}{|c|}{ Natriumchlorid $\left(\rho=2164 \mathrm{~kg} / \mathrm{m}^{3}\right.$ und $\left.a=5.633 \AA\right)$} \\
\hline elast. Konstanten [GPa] & $\begin{array}{r}c_{11} \\
49.1\end{array}$ & $\begin{array}{r}c_{12} \\
12.8\end{array}$ & $\begin{array}{r}c_{44} \\
12.8\end{array}$ \\
\hline Temperaturkoeffizient $\left[10^{-4} 1 / \mathrm{K}\right]$ & -7.8 & +4.7 & -2.2 \\
\hline Druckkoeffizient $\left[10^{-12} 1 / \mathrm{Pa}\right]$ & 239 & 183 & 29 \\
\hline & $\mathrm{TA}\left[\begin{array}{lll}1 & 0 & 0\end{array}\right]$ & $\mathrm{TA}\left[\begin{array}{lll}1 & 1 & 0\end{array}\right]$ & $\mathrm{TA}\left[\begin{array}{lll}1 & 1 & 1\end{array}\right]$ \\
\hline Kombination der elast. Konst. [GPa] & 12.8 & 12.8 & 49.1 \\
\hline$v_{\text {Schall }}[\mathrm{m} / \mathrm{s}]$ & 2432 & 2432 & 2750 \\
\hline$(\partial v / \partial \xi)[\mathrm{THz}]$ & 4.32 & 6.11 & 8.46 \\
\hline & LA $\left[\begin{array}{lll}1 & 0 & 0\end{array}\right]$ & LA $\left[\begin{array}{lll}1 & 1 & 0\end{array}\right]$ & $\mathrm{LA}\left[\begin{array}{lll}1 & 1 & 1\end{array}\right]$ \\
\hline Kombination der elast. Konst. [GPa] & 49.1 & 87.5 & 126 \\
\hline$v_{\text {Schall }}[\mathrm{m} / \mathrm{s}]$ & 4763 & 4496 & 4404 \\
\hline$(\partial v / \partial \xi)[\mathrm{THz}]$ & 8.46 & 11.3 & 13.5 \\
\hline
\end{tabular}


manuell angepaßt wurden. Dabei wurde versucht, insbesondere die experimentell bestimmte Dispersion für die TA Phononen mit dem Modell wiederzugeben, weil sie für diese Arbeit von besonderer Bedeutung sind. Es konnte aber ein solcher Parametersatz nicht gefunden werden.

Um Entmischungsprozesse zu verfolgen, bieten sich die TA Phononen an. Sie entsprechen in Silber- und Natriumchlorid dem niederenergetischsten Dispersionszweig und haben in einem inelastischen Neutronenstreuexperiment die größte zu erwartende Intensität. Genauso werden die Experimente bei kleinen Wellenvektoren $q$ durchführt, um eine möglichst große Intensität zu erhalten. Von den TA Phononen ist dann das TA Phonon in $\left[\begin{array}{lll}1 & 0 & 0\end{array}\right]$ bzw. in $\left[\begin{array}{lll}0 & 1 & 1\end{array}\right]$ zu bevorzugen, weil die Dispersion der reinen Substanzen für $q \rightarrow 0$ sich um ca. $10 \%$ stärker unterscheiden als in [ $\left.\begin{array}{lll}1 & 1 & 1\end{array}\right]$; vgl. Tabelle 2.1. Darüber hinaus lassen sich die Probenkristalle in der $\left[\begin{array}{lll}1 & 0 & 0\end{array}\right]$-Ebene sehr gut spalten. Das ist mit Hinblick auf die Experimente von Vorteil, weil Proben in Würfel- oder Quaderform sich leichter handhaben lassen.

In einem inelastischen Neutronenstreuexperiment ist dann unter Berücksichtigung der vorherigen Arbeiten folgendes zu erwarten: In der homogenen Phasen wird die Dispersion zwischen den Dispersionen der reinen Substanzen verlaufen. Durch das Abkühlen von $400{ }^{\circ} \mathrm{C}$ auf $100{ }^{\circ} \mathrm{C}$ sollte die Steigung der Dispersion für $q \rightarrow 0 \mathrm{um}$ $4 \%$ zunehmen. Entmischt die Probe spinodal, bilden sich mit der Zeit kontinuierliche, periodische Konzentrationsprofile aus. Wird nun ein Phononenspektrum bei konstantem $q$ gemessen, sollte das Spektrum der homogenen Phase in eine Frequenzverteilung übergehen, die die Konzentrationsverteilung widerspiegelt. Im Gleichgewichtszustand liegen dann zwei definierte Phononen für die jeweils $\mathrm{Na}$ - und $\mathrm{Ag}$-reiche Phase vor. Entmischt die Probe nach dem Mechanismus der Keimbildung und des Keimwachstums, muß das anfängliche Spektrum mit einem Phonon in ein Spektrum mit zwischenzeitlich drei und am Ende zwei Phononen übergehen. Das zur ursprünglich homogenen Phase gehörende Phonon sollte an Intensität ab- und die beiden anderen Phononen zunehmen, während sich die Phononenfrequenz bei allen drei Phononen nicht ändert. Bei beiden Mechanismen können die Phononen gedämpft sein. Zeitlich muß sich das Spektrum in Analogie zu den SANS-Experimenten innerhalb von $1-2 \cdot 10^{2}$ s deutlich ändern. 


\section{Experimente}

\subsection{Probenpräparation}

In allen Experimenten wurde Silberchlorid (99.9\%) der Firmen Alfa und PureChem sowie Natriumchlorid ( $>99.5 \%$ ) der Firma Merck verwendet. Die Mischungen wurden direkt vor der jeweiligen Probenpräparation eingewogen und gemischt. Die Präparationen und alle Arbeiten an den Proben erfolgten bei Gelblicht. Um die Qualität der Proben und des Silberchlorids in angebrochenen Verpackungen zu erhalten, wurden sie unter Stickstoffatmosphäre verpackt und trocken (Silicagel) bei Raumtemperatur und unter Lichtausschluß gelagert.

Alle verwendeten Pulverproben wurden durch Zusammenschmelzen von reinem Silberchlorid und Natriumchlorid unter $\mathrm{N}_{2}$-Schutzgasatmosphäre hergestellt. Hierzu wurden die für die jeweilige Zusammensetzung benötigten Einwaagen in Porzellan oder Quarzglasgefäßen aufgeschmolzen oder das Natriumchlorid in flüssigem Silberchlorid gelöst. Der Schmelzkuchen wurde in einem Achatmörser in einer Kugelmühle oder per Hand gemörsert und durch fraktioniertes Sieben auf die gewünschte Korngröße eingestellt. In den Röntgendiffraktionsexperimenten zur Konzentrationsbestimmung, vgl. Seite 41, wurden ausschließlich Fraktionen mit einer Korngröße $d \leq 20 \mu \mathrm{m}$ verwendet. Bei den Neutronenstreuexperimenten sind Fraktionen von bis zu $d \leq 200 \mu$ m verwendet worden, die zu Tabletten gepreßt wurden. Dabei wurden in Vorversuchen keine Hinweise auf Einflüsse durch das Pressen gefunden.

Voraussetzung für die inelastischen Neutronenstreuexperimente sind Silber-Natriumchlorid-Einkristalle mit der gewünschten mittleren Zusammensetzung in einer ausreichenden Qualität. In vorangegangenen Arbeiten [Windgasse 1994] konnte gezeigt werden, daß sich Einkristalle des analogen Silber-Natriumbromidsystem nach dem Czochralski-Verfahren ${ }^{1}$, vgl. Abbildung 3.1, herstellen lassen. Bei diesem Verfahren wird ein Tiegel mit der Probensubstanz gefüllt. Durch eine Tischheizung und eine Umgebungsheizung wird die Probensubstanz aufgeschmolzen. Von oben wird ein Impfkristall, der sich am Ende einer sich drehenden und gekühlte Ziehwelle befindet, in die Schmelze eingetaucht. Nach dem Ankristallisieren der Schmelze an dem Impfkristall wird dieser langsam angehoben, so daß Schmelze immer weiter nachkristallisieren kann. Das Czochralski-Verfahren wird zur Züchtung von Einkristallen mit nur einer Komponente oder von Mehrkomponentensystemen mit kongruenten Schmelzpunkt wie

\footnotetext{
${ }^{1}$ Allgemein zur Kristallzucht sei hierzu u. a. auf [Pamplin 1980, Hurle 1993] verwiesen.
} 


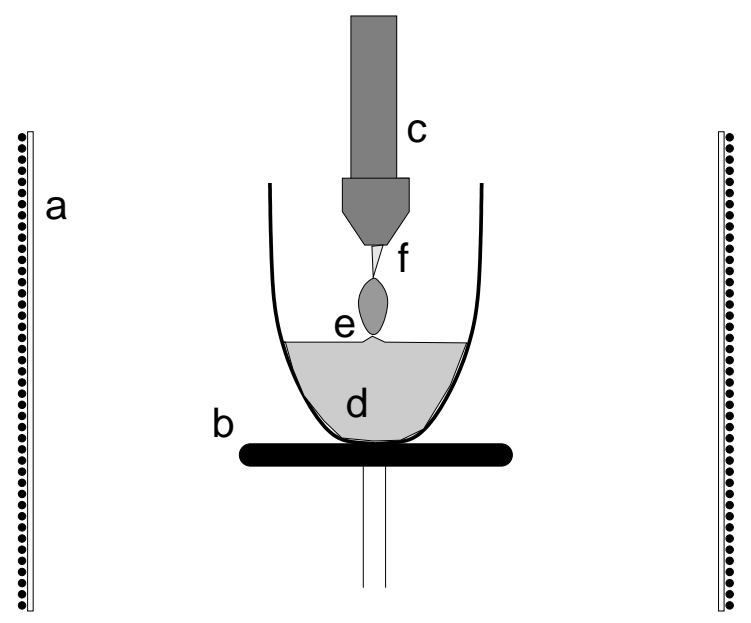

Abbildung 3.1: Schematische Darstellung der Kristallzucht nach dem Czochralski-Verfahren: a) Umgebungsheizung, b) Tischheizung, c) Ziehwelle, d) Schmelze, e) Kristall, f) Impfkristall

z.B. Halbleiterlegierungen oder viele Mischoxide verwendet und ist dann ein isothermer Prozeß. Das Czochralski-Verfahren konnte erfolgreich auf das Silber-Natriumchlorid System übertragen werden. Aus der Literatur [Stokes und Li 1962] ist zudem bekannt, daß es möglich ist, kleine Silber-Natriumchlorid Mischkristalle zu entmischen, ohne daß sie von außen sichtbare Schäden zeigen.

Im Gegensatz zu anderen Kristallsystemen mit einem kongruenten Schmelzpunkt ist das isotherme Züchten von Silber-Natriumchlorid Einkristallen nicht möglich: Wie in Abbildung 3.2 zu erkennen ist, müßte hierzu die Zusammensetzung der Schmelze konstant sein. Ansonsten kommt es zu einer Verarmung an Natriumchlorid in der Schmelze, und die Zusammensetzung des Kristalls ändert sich. Hinzu kommt eine Verringerung der Schmelztemperatur aufgrund der Natriumchloridverarmung. Das Absenken der Schmelztemperatur zieht eine weitere Konsequenz nach sich: Die Wachstumsrate der Kristalle wird durch den Wärmeabfluß aus dem Kristall bestimmt. Wenn ein Kristall eine kritische Größe erreicht hat, ist der Wärmeabfluß nicht mehr ausreichend, damit sich weiter Substanz kristallisiert, und der Kristall reißt von der Schmelze ab.

Zunächst wurde versucht, die Kristalle möglichst isotherm zu züchten. Die so erhaltenen Kristalle hatten aber aus den oben genannten Gründen keine für Neutronenstreuexperimente ausreichende Größe. So wurde durch Variation der Schmelzansätze und der Temperaturprofile versucht, große, möglichst homogene Einkristalle zu züchten. Aus verschiedenen Experimenten mit der Kristallzucht hat sich folgendes Vorgehen als beste Variante herausgestellt:

Die Zusammensetzung der Schmelze wird durch die gewünschte mittlere Zusammensetzung des Kristalls vorgegeben und kann anhand des Phasengleichgewichts (s)(l) bestimmt werden. Um der Natriumchloridverarmung entgegen zu wirken, wird der 


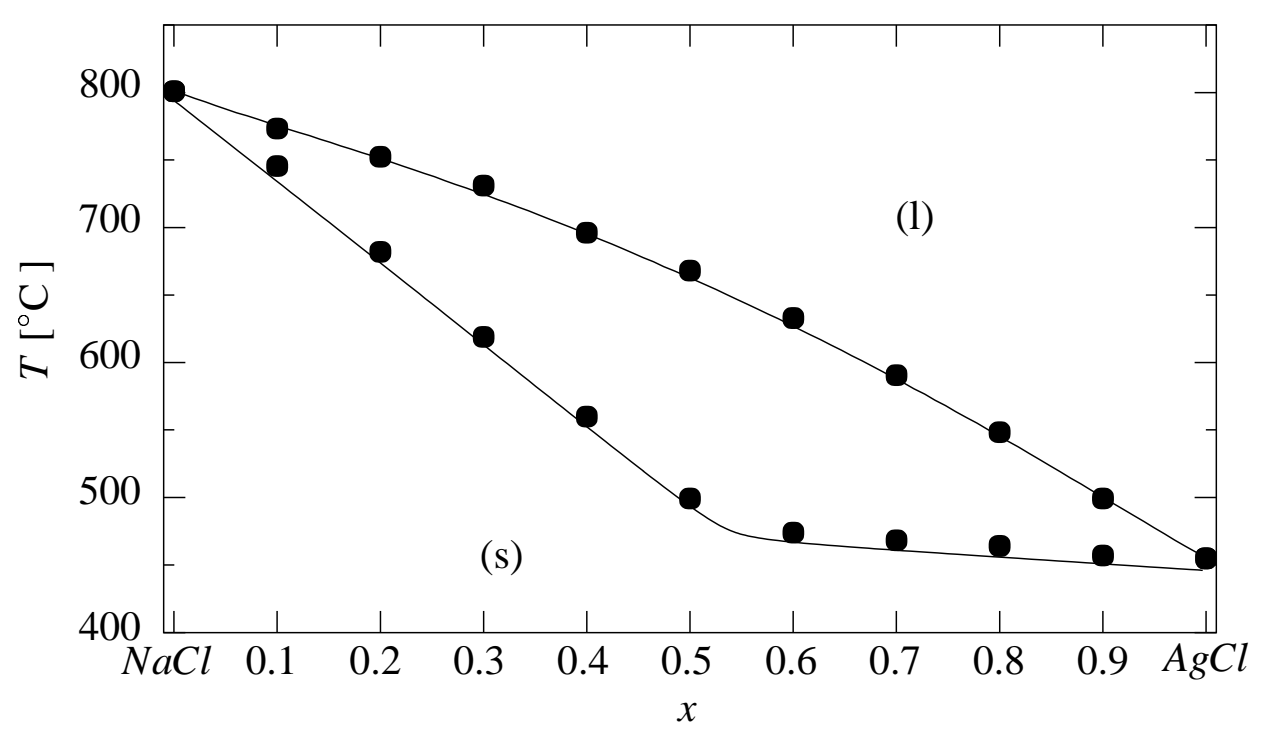

Abbildung 3.2: Das (s)-(1)-Gleichgewicht des $\mathrm{Ag}_{x} N a_{1-x} \mathrm{Cl}$-Systems

Silberchloridanteil in Abhängigkeit von der gewünschten Kristallgröße erniedrigt. Für Kristalle mit $x_{0} \approx 0.5$ ergaben Experimentreihen mit der vorhandenen Kristallzuchtanlage, daß Schmelzen mit einem Silberchloridanteil von 80-90 \% und einer Masse von 100-120 g angesetzt werden müssen. Als Schmelztiegel wurden konische FZY-Tiegel der Firma Friatec, Mannheim, angesetzt. Als Impfkristalle wurden konische $\mathrm{NaCl}$-Kristalle der Firma Korth Kristalle GmbH sowie Kristallite aus vorangegangenen Kristallzuchtversuchen verwendet. Die kommerziellen $\mathrm{NaCl}$-Kristalle waren parallel zu [110]Ebenen geschnitten. Die selbst präparierten Impfkristalle wurden parallel zur [100]Ebene gespalten und so in die Keimkristallhalterung eingesetzt, daß die Zugachse parallel zur [110]-Ebene lag. Bei der Auswahl von Mischkristallen als Impfkristall ist auf die mittlere Zusammensetzung des Impfkristalls zu achten, da er sich sonst in der Schmelze auflösen kann.

Nach dem Einbau des Impfkristalls und des Schmelzansatzes in die Kristallzuchtapparatur wurde diese mindestens fünf Mal mit Stickstoffgas gespült. Der anfängliche Druck in der Apparatur betrug bei Raumtemperatur in der Regel ca. $61 \mathrm{kPa}$. Die Umgebungsheizung wurde auf eine Temperatur von $10-15^{\circ} \mathrm{C}$ oberhalb der nach dem Phasendiagramm zu erwartenden Schmelztemperatur eingestellt. Die Heizleistung der Tischheizung wurde dann schrittweise erhöht, bis der gesamte Schmelzansatz verflüssigt war. In der Schmelze waren immer einige dunkle, unlösliche Schwebstoffe vorhanden, die jedoch in keinem der erhaltenen Kristalle wiederzufinden waren. Die Leistung der Tischheizung wurde nun erniedrigt, bis nur noch eine sehr schwache, an den Schwebstoffen zu erkennende Konvektion in der Schmelze zu beobachten war. Der 
Impfkristall wurde abgesenkt, bis er sich 1-2 mm über die Schmelze befand, und einige Minuten gewartet. Damit wurde ein spontanes Kristallisieren an einem zu kalten Impfkristall verhindert. Die Spitze des sich drehenden Impfkristalls wurde dann 1-2 mm in die Schmelze eingetaucht. Die Drehgeschwindigkeit betrug eine Umdrehung pro $26 \mathrm{~s}$. Nach ca. 5 min wurde der Impfkristall aus der Schmelze herausgezogen, so daß sich ein sichtbarer Meniskus zwischen der Schmelze und dem Impfkristall herausbildete. Nun wurde der Kristall mit ca. $1.5 \mathrm{~mm} / \mathrm{h}$ angehoben, um eine Keimauswahl zu treffen . Die Ziehgeschwindigkeit wurde danach schrittweise auf weniger als $0.3 \mathrm{~mm} / \mathrm{h}$ abgesenkt.

Bei den ersten Kristallen wurde versucht, durch Variation der Zuggeschwindigkeit einen Hals zu ziehen, um so eine Keimauswahl zu treffen. Dies erwies sich aber als sehr schwierig, da der Kristall immer wieder von der Schmelze abriß. Zudem hatte die Keimauswahl keinen merklichen Einfluß auf die Probenqualität, so daß darauf verzichtet wurde.

Der sich verschlechternde Wärmeabfluß machte sich durch abnehmende Kristalldurchmesser bemerkbar. Um das Abreißen des Kristalls von der Schmelze zu verhindern, wurde die Temperatur der Tischheizung vorsichtig schrittweise erniedrigt. Wenn der Kristall eine bestimmte Größe erreicht hatte, die einer Masse von ca. knapp $10 \mathrm{~g}$ entspricht, wurde die Temperatur nicht mehr nachgeregelt. Der Kristall wurde dann mindestens über $24 \mathrm{~h}$ bei dieser Temperatur gehalten, um evtl. Konzentrationsgradienten innerhalb des Kristalls auszugleichen.

Zusammenfassend kann man bezüglich der Kristallzuchtbedingungen und der resultierenden Kristallqualität feststellen, daß die Qualität der Kristalle mit

1. der Vergrößerung des Schmelzansatzes,

2. $A g_{x} N a_{1-x} C l$-Impfkristallen,

3. kleineren, spitzen Impfkristallen,

4. abnehmender Ziehgeschwindigkeit,

5. abnehmender Drehgeschwindigkeit,

6. abnehmender Temperaturkorrektur,

7. zunehmender Länge des Kristalls und

8. abnehmender Querschnittsfläche des Kristalls

zunimmt. Es ist bemerkenswert, daß die trockene Lagerung der Kristalle bei Raumtemperatur über Zeitraum von mehr als einem Jahr keinen oder keinen bisher nachweisbaren Einfluß auf die Kristallqualität hat.

Schon kurz nach der Entnahme aus der Kristallzuchtanlage setzte eine mit Auge sichtbare Trübung der Kristalle ein, wie sie schon in der Literatur [Stokes und Li 1962] 
beschrieben wurde. Waren die Kristalle anfänglich noch klar, wie es besonders gut an dem durchleuchteten Foto in Abbildung $3.3 \mathrm{zu}$ sehen ist, nahm die Trübung mit der Zeit deutlich zu. Die Kristalle mit $x_{0}=0.23$ und $x_{0}=0.26$ nahmen eine weiße Farbe an, während der Kristall mit $x_{0}=0.41$ sich nur eintrübte. Wenn die Kristalle wieder auf Temperaturen oberhalb von $200{ }^{\circ} \mathrm{C}$ erhitzt wurden, verloren sie ihre Trübung.

Um die Qualität der Kristalle zu kontrollieren, wurden an einem Gammadiffraktometer [Elisbihani 2002] die Mosaizitäten der Kristalle gemessen: Hierfür wird in einem Diffraktometer die Bragg-Bedingung für einen Reflex eingestellt, und der Intensitätsverlauf als Funktion des Probendrehwinkels in der Streuebene gemessen. Die Halbwertsbreite des Intensitätsverlaufs als Funktion des Drehwinkels ist die Mosaizität des Kristalls, die ein Maß für die Kristallperfektion ist; je kleiner die Mosaizität, desto perfekter ist das Kristallgitter. Die Mosaizität wurde an mindestens zwei Braggreflexen und jeweils einem symmetrieäquivalenten Reflex gemessen. Es waren in der Regel der (2 0 0)- und (2 20 )-Reflex. Im Vergleich mit kommerziellen $\mathrm{NaCl}$-Einkristallen, die eine Mosaizität von einigen Zehntel Grad besitzen, ist festzustellen, daß die Mischkristalle mit Mosaizitäten von $1.3^{\circ}$ und weniger von sehr guter Qualität sind.

Um die mittlere Zusammensetzung der Kristalle zu bestimmen, sind zwei Methoden parallel angewandt worden. Zum einen wurden atomemmissionsspektroskopische Analysen vom Analytischen Labor des Instituts für Anorganische Chemie und von der Zentralabteilung für chemische Analysen des Forschungszentrums Jülich durchgeführt. Zum anderen wurde die Zusammensetzung durch Röntgendiffraktion bestimmt. Hierzu wurden die Proben auf Korngrößen kleiner $0.02 \mathrm{~mm}$ gemörsert und bei $400{ }^{\circ} \mathrm{C}$ homogenisiert. Bei dieser Temperatur wurde dann ein ein Diffraktogramm aufgenommen und die Gitterkonstante aus einer Auftragung von $\sin ^{2}(\Theta)$ vs. $\left(h^{2}+k^{2}+l^{2}\right)$ bestimmt. Die mittlere Zusammensetzung ergibt sich dann nach der Vegardschen Regel zu

$$
x=\frac{a_{\text {Probe }}-a_{N a C l}}{a_{A g C l}-a_{N a C l}} .
$$

Für die Analysen wurden jeweils zwei bis drei Proben entlang der Wachstumsrichtung der Kristalle genommen. Damit konnte der Kristall auf eventuelle Konzentrationsgradienten hin untersucht werden.

Die Kristalle wurden in den Experimenten zunächst bei Raumtemperatur statisch, wie sie nach der Kristallzucht vorlagen, untersucht. Nach statischen Messungen im homogenen Bereich des Phasendiagramms platzten sie in kleinere Fragmentstücke, wenn die Kristalle zum ersten Mal zügig von hohen Temperaturen abgekühlt wurden. Aus diesen Fragmentstücken wurden die Proben für die dynamischen Experimente ausgewählt. Dabei mußte darauf geachtet werden, daß die Kristalle zwar ein ausreichendes Streuvolumen für die Neutronenstreuexperimente besitzen, aber bzgl. der Entmischungskinetik noch immer schnell genug abgekühlt werden können. Dabei hat sich in den Experimenten ein Streuvolumen von ca. $0.4-0.8 \mathrm{~cm}^{3}$ als günstig erwiesen. Im Rahmen dieser Arbeit wurden folgende Kristalle verwendet: 

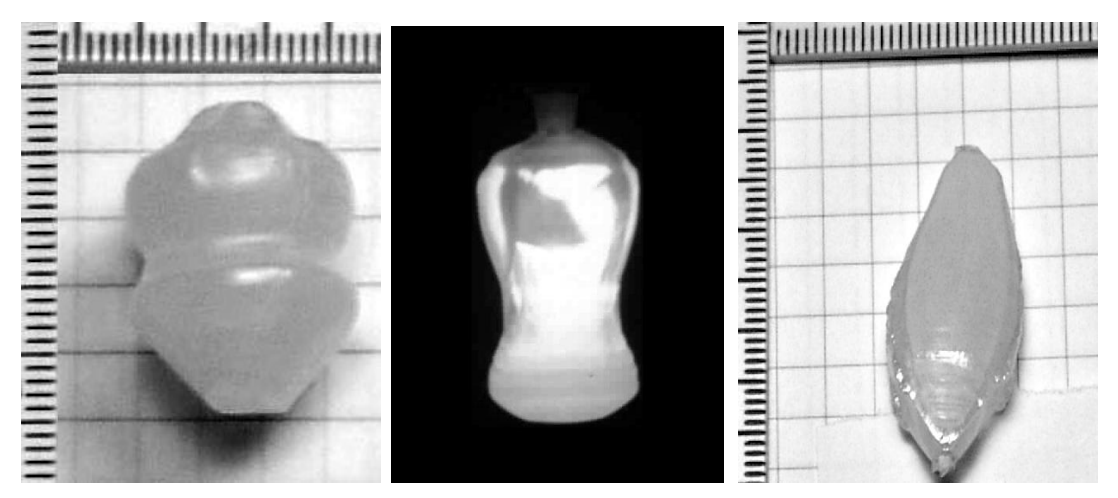

Kristall I, $Q=\left(\begin{array}{lll}2 & 2 & 0\end{array}\right) \quad$ Kristall II, $Q=\left(\begin{array}{lll}2 & 0 & 0\end{array}\right)$

Kristall III, $Q=\left(\begin{array}{lll}2 & 0 & 0\end{array}\right)$
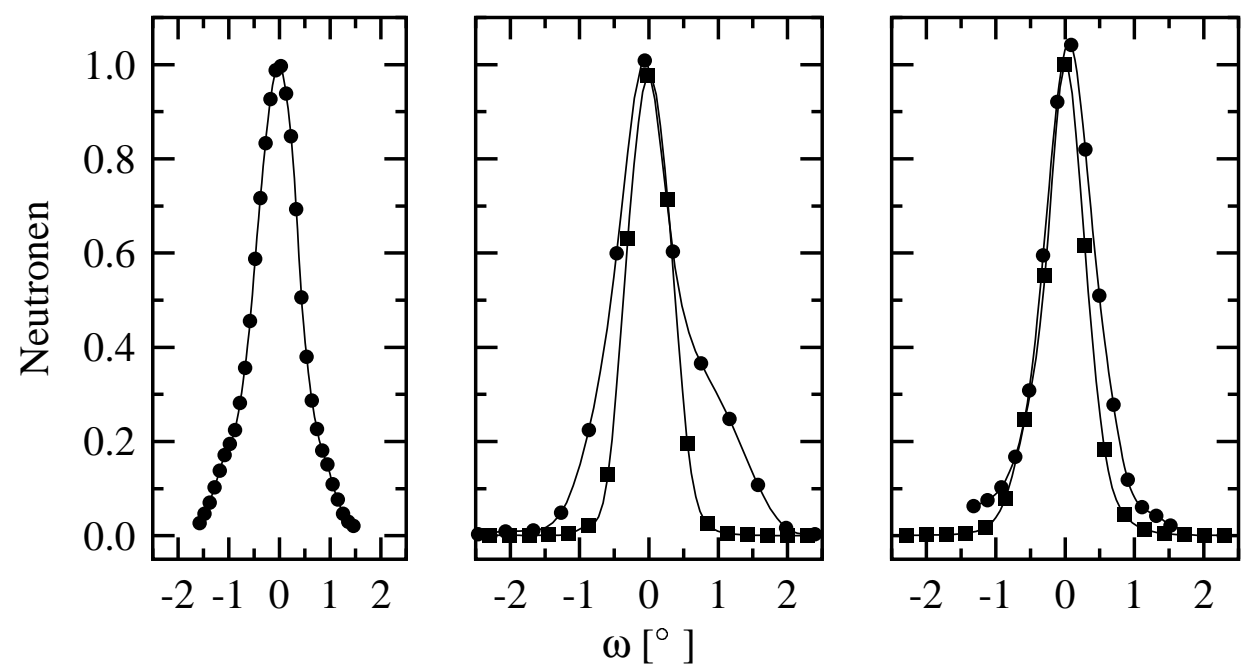

Abbildung 3.3: Die Fotos zeigen die Probenkristalle kurz nach der Entnahme aus der Kristallzuchtanlage. Die in den Fotos am Rand abgebildeten Längeneinheiten entsprechen $1 \mathrm{~mm}$. Der Kristall II ist durchleuchtet fotografiert: Es ist am oberen Ende der Keimkristall und im oberen Drittel deutlich eine Spaltfläche innerhalb des Kristalls zu erkennen. Die untere Reihe zeigt die jeweilige Diffraktogramme zur Bestimmung der Mosaizität. Für die Kristalle II und III sind zum Vergleich zusätzlich die schmaleren Kurven für ein Fragmentstücke mit aufgetragen. 
Kristall I Der Kristall (vgl. Abbildung 3.3) hatte eine Länge von 23 mm, ein Gewicht von ca. $8 \mathrm{~g}$ und eine Mosaizität von $1.1^{\circ}$. Die Bestimmung aus der Gitterkonstanten ergab eine Zusammensetzung von $x_{0}=0.22$. Die atomemmissionsspektroskopische Analyse mit Silber lieferte in guter Übereinstimmung $x_{0}=0.23 \pm 0.02$. Die Analyse mit Natrium ermittelte jedoch eine mittlere Zusammensetzung von $x_{0}=0.28 \pm 0.02$. Da aber im Vergleich der Experimentergebnisse der Kristall keinen höheren Silberanteil als der Kristall II haben kann, wurde dieses Analyseergebnis verworfen. Das falsche Ergebnis kann dadurch erklärt werden, daß unbemerkt kleine Mengen beim Lösen der Proben in $K S C N_{(\mathrm{aq})}$ nicht in Lösung gingen. Das in den dynamischen Experimenten verwendete Fragmentstück hatte eine Mosaizität von $\eta \approx 1.0^{\circ}$ und eine Größe von $7 \times 8 \times 13 \mathrm{~mm}^{3}$.

Kristall II Der Kristall hatte eine Länge von $25 \mathrm{~mm}$, eine Masse von $8.8 \mathrm{~g}$ und eine Mosaizität von $1.3^{\circ}$. Die Analysen ergaben in guter Übereinstimmung für die mittlere Zusammensetzung $x_{0}=0.26$. Das in den dynamischen Experimenten verwendete Fragmentstück hatte eine Größe von $8 \times 7 \times 13 \mathrm{~mm}^{3}$ und eine Mosaizität von $0.9^{\circ}$.

Kristall III Der Kristall hatte eine Länge von $30 \mathrm{~mm}$, eine Masse von ca. $10 \mathrm{~g}$ und eine Mosaizität von $0.75^{\circ}$. Beide Analysemethoden lieferten in guter Übereinstimmung eine mittlere Zusammensetzung von $x_{0}=0.41$. In den dynamischen Experimenten wurden Fragmentstücke mit Mosaizitäten von $1^{\circ}$ verwendet. Die Größe der Fragmente war $5 \times 7 \times 9 \mathrm{~mm}^{3}$ und $5 \times 7 \times 11 \mathrm{~mm}^{3}$.

\subsection{Neutronenstreuexperimente}

Die Methode der Wahl zur Untersuchung von Gitterschwingungen ist die inelastische Neutronenstreuung. Derartige Experimente werden an Dreiachsenspektrometern durchgeführt, mit denen im Gegensatz zu Diffraktometern sich auch Energieänderungen der Neutronen messen lassen. Die Neutronenstreuexperimente wurden am UNIDAS, Forschungszentrum Jülich, und am IN-12, Institut Laue Langevin (ILL), Grenoble (F), durchgeführt. Zur Erprobung der Zeitauflösung, siehe Abschnitt 3.4, wurde ein Vorversuch am IN-3, ILL, durchgeführt.

Der prinzipielle Aufbau eines Dreiachsspektrometers ist in Abbildung 3.4 skizziert. Die Neutronen werden mittels Kernspaltung im Reaktor (R) erzeugt und mit einem Strahlrohr aus dem Reaktor durch die Abschirmung geführt. Dabei wird der Neutronenstrahl bereits durch das Strahlrohr oder dem Neutronenleiter kollimiert (K1). Dann wird der Neutronenstrahl monochromatisiert (M,K2). Dabei wird aus der Energieverteilung der Neutronen, dem Reaktorspektrum, die gewünschte Wellenläge herausgefiltert, bevor der Neutrononstrahl auf die Probe (P) trifft und gestreut wird. Mit dem Kollimator 


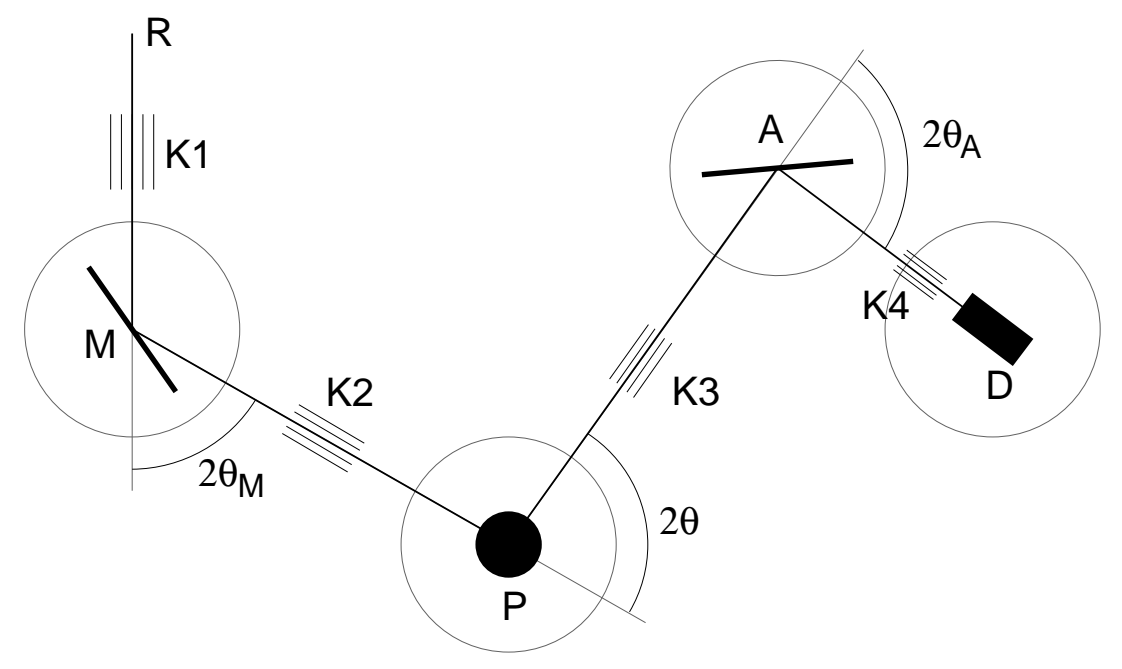

Abbildung 3.4: Schematischer Aufbau eines Neutronendreiachsspektrometers

K3 wird der gestreute Strahl bezüglich des zu untersuchenden Raumwinkels selektiert, die Energie der Neutronen analysiert (A, K4) und im Detektor (D) nachgewiesen. Die Energie der Neutronen wird im Monochromator und im Analysator durch die energieabhängige Beugung an einem Einkristall selektiert. Um Schwankungen des Neutronenflusses und die Charakteristik von Monochromator oder Analysator zu korrigieren, muß die Intensität normiert werden. So ist z. B. die Reflektivität von pyrolytischem Graphit, der oft als Monochromator und Analysatormaterial eingesetzt wird, energieabhängig; vgl. Abbildung .4 auf Seite 105. Es befinden sich hierfür Neutronendetektoren mit sehr kleinen Ansprechwahrscheinlichkeiten, sogenannte Monitore, vor und hinter der Probe. Abbildung 3.5 zeigt den Versuchsaufbau am IN-12.

Die endliche Auflösung des Spektrometers führt dazu, daß $k_{\mathrm{i}}$ und $k_{\mathrm{f}}$ nicht beliebig gut definiert sind, wie in Abbildung 3.6 überzeichnet dargestellt. Daraus ergeben sich folglich Verteilungen von $\mathbf{Q}$ und $\omega$. Im Experimenten wird demzufolge nicht direkt die Streufunktion $S(\mathbf{Q}, \omega)$ gemessen, sondern die Faltung der Streufunktion mit Auflösungsfunktion $R(\mathbf{Q}, \omega)$ des Spektrometers, die in der Regel gaußisch angenähert werden kann:

$$
I(\mathbf{Q}, \omega)=\iint R\left(\mathbf{Q}-\mathbf{Q}_{0}, \omega-\omega_{0}, \mathbf{Q}, \omega\right) S\left(\mathbf{Q}_{0}, \omega_{0}\right) \mathrm{d} \mathbf{Q}_{0} \mathrm{~d} \omega_{0}
$$

Mit Kenntnis der Auflösungsfunktion des Spektrometers ${ }^{2}$ lassen sich die gerätebedingten Halbwertsbreiten parallel $\Delta_{\| Q}$ und senkrecht $\Delta_{\perp Q}$ zum Streuvektor und die Halbwertsbreite in den Spektren $\Delta_{V}$ berechnen. Oder es kann aus $I(\mathbf{Q}, \omega)$ durch Entfaltung $S(\mathbf{Q}, \omega)$ bestimmt werden.

\footnotetext{
${ }^{2}$ siehe u. a. [Cooper und Nathans 1967, Eckold und Mitlacher 1982, A.Bouvet und A.Filhol 1995, A.Bouvet u. a. 1997].
} 


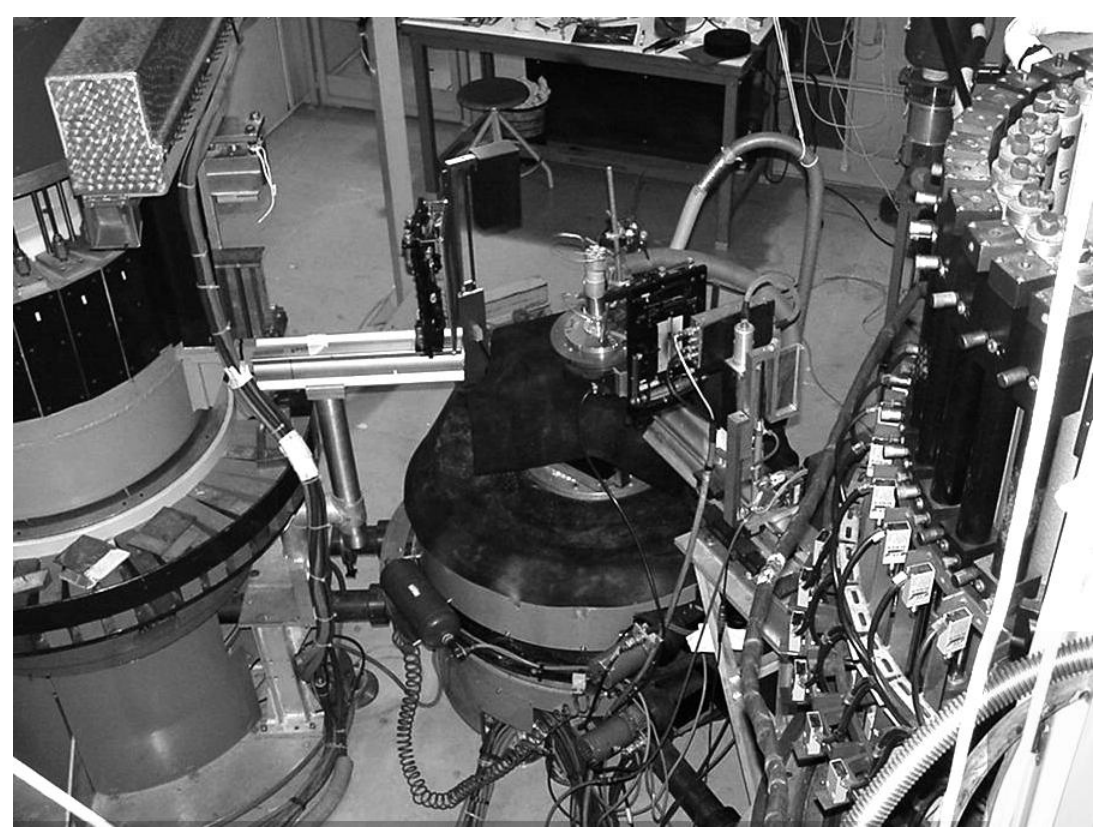

Abbildung 3.5: Der Probenaufbau am IN-12: In der Mitte des Bildes steht der Probentisch mit dem Ofen. Am rechten Rand ist der Monochromator, am linken Rand der Analysator zu sehen. Die zwei optischen Bänke vom Monochromator zum Probentisch und vom Probentisch zum Analysator stehen parallel zu $k_{\mathrm{i}}$ bzw. $k_{\mathrm{f}}$. Am Ende der optischen Bänke, die dem Ofen zugewandt sind, ist jeweils ein Blendensystem befestigt. Auf der Bank zwischen dem Monochromator und dem Ofen sind zusätzlich ein Abschwächer für den Primärstrahl und ein Monitor angebracht.

\subsection{Probenöfen für die Neutronenstreuexperimente}

Ein Vorteil der Neutronenstreuung ist die hohe Eindringtiefe von Neutronen, die komplexe Probenumgebungen wie Öfen ermöglicht. Als Ofenmaterial für die kinetischen Experimente eignet sich Aluminium, weil es eine geringe Wärmekapazität hat und aufgrund seiner geringen Dichte und kleinen Streulänge kaum mit Neutronen wechselwirkt ${ }^{3}$.

Für die kinetischen Experimente wurden vorhandene Öfen für statische Messungen weiterentwickelt. Bei der Ofenentwicklung spielten folgende Kriterien eine entscheidend Rolle:

1. maximale Kühlraten durch aktive Kühlung der Probe

2. definiertes Abkühlprofil ohne Über- oder Unterschwingen der Temperatur

3. Temperaturstabilität über die gesamte Meßzeit

\footnotetext{
${ }^{3}$ Darüber hinaus hat Aluminium den Vorteil, daß es von Neutronen nur schwach aktiviert wird und aufgrund der kurzen Halbwertszeit von 2.25 min während der Neutronenstreuexperimente problemlos am Ofen gearbeitet werden kann.
} 


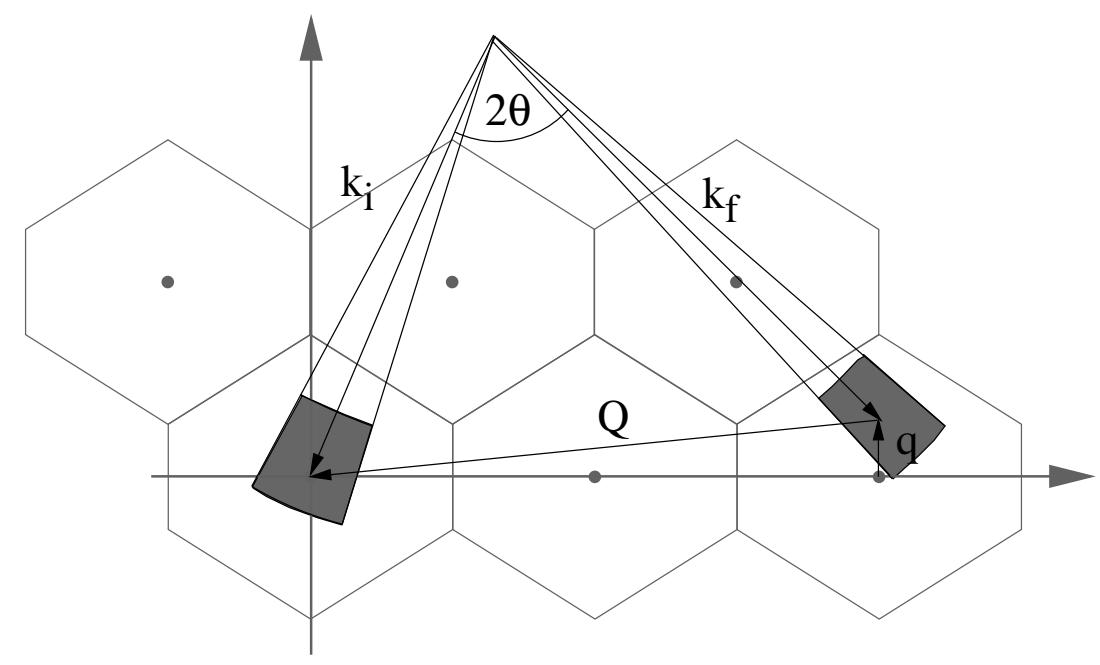

Abbildung 3.6: Aufgrund der endlichen Auflösung des Spektrometers kommt es zu einer Verteilung von $k_{\mathrm{i}}, k_{\mathrm{f}}, Q$ und $\omega$, die als graue Flächen dargestellt ist.

4. möglichst keine Temperaturgradienten in der Probe

5. mechanisch stabile Fixierung der Probe trotz der periodisch auftretenden Temperaturänderungen von $300{ }^{\circ} \mathrm{C}$

6. Temperaturwechselbeständigkeit des Ofens

7. geringer Meßuntergrund durch den Ofen

8. möglichst geringe Aktivierung des Ofens durch Neutronen

Abbildung 3.7 links zeigt ein Foto des ersten Ofens. Bei diesem Ofen handelt es sich im Prinzip um ein Aluminiumzylinder (a) mit jeweils einer Heizpatrone (b) am oberen und unteren Ende. Am unteren Ende (c) ist der Zylinder mit Schrauben an einem Stenanstab befestigt. Hier befinden sich zudem Bohrungen, um Luft von unten in den Ofen einblasen zu können. In diesem Ofen wurde erfolglos versucht, die Probe mit Sauereisenzement oder Keramikkleber auf den Stenanstab zu fixieren: Bei den auftretenden Temperaturänderungen löste sich der Kristall immer wieder. Es wurde außerdem versucht, den Kristall zusätzlich mit Glaswolle im Ofen zu stabilisieren. Dies hatte nur mäßigen Erfolg und verschlechterte die Abkühlraten deutlich. Die erzielten Abkühlraten waren aber zu gering, um mit diesem Ofen kinetische Experimente durchzuführen.

Der erste zu verbessernde Punkt war die Trägheit des Ofens, die maßgeblich durch die verwendeten Heizpatronen gegeben war. In Abbildung 3.7 rechts und Abbildung 3.8 ist der optimierte Neutronenofen gezeigt. Dieser Ofen besteht wieder aus Aluminium. Als Heizelemente werden zwei Mantelheizleiter mit kalten Enden vom Typ SEI 20100 der Firma Thermocoax in einer Nut auf dem Aluminiumzylinder verstemmt (a). 

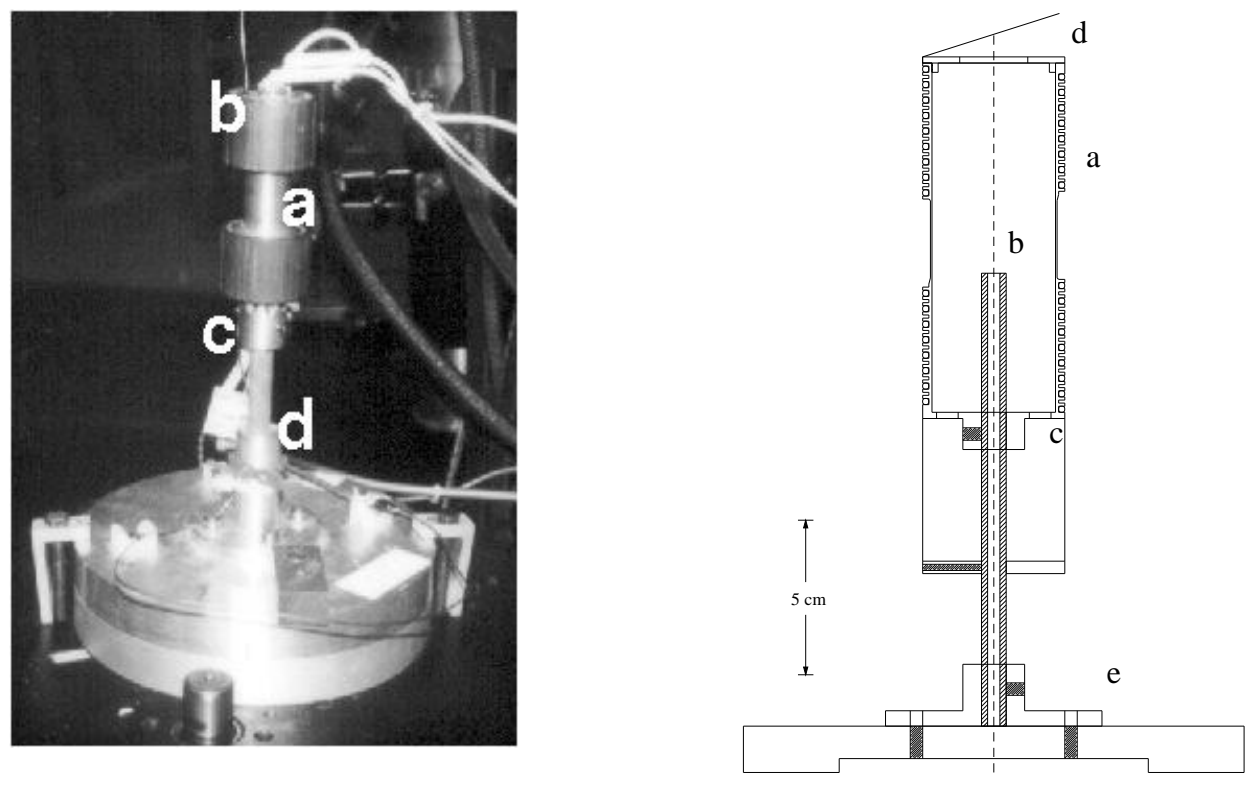

Abbildung 3.7: Der erste Neutronenofen (links) und eine Schnittzeichnung des in den Experimenten verwendeten Ofens (rechts)

Die Probe wird auf einem Degussitrohr (b) mit einem Durchmesser von $8 \mathrm{~mm}$ und einer Wandstärke von $1.5 \mathrm{~mm}$ befestigt. In der Bodenplatte des Ofens befinden sich Bohrungen, durch die mit Hilfe einer Umlenkhülse (c) Luft von unten in den Ofen eingeblasen wird. Durch den Luftstrom wird ein Lüftungsventil im Deckel (d) geöffnet, damit die heiße Luft entweichen kann. Ist die jeweilige Solltemperatur erreicht, wird der Deckel geschlossen. Durch den Deckel ist das Temperaturfeld im Ofen überdies deutlich homogener. Um den Ofen mechanisch so stabil wie möglich zu halten, werden für alle Halterung am Degussitstab Schrauben mit gefederter Kugelspitze verwendet. Der Degussitstab selbst hat jeweils am oberen und unteren Ende zwei um $120^{\circ}$ versetzte Kerben, in denen die Kugelspitzen einrasten. So wird ein Verdrehen des Ofens gegen den Degussitstab oder in der Grundplatte (e) verhindert. Vor dem Experimentbetrieb ist der Ofen auf die maximale Temperatur aufzuheizen und alle Halteschrauben sind nachzuziehen.

Um die Probenkristalle zu befestigen, wird $0.025 \mathrm{~mm}$ dicke Silberfolie in geeignet große Rechtecke geschnitten ${ }^{4}$ und eine scharfe Kante durch die Folie gezogen. Die Folie wird zwei bis vier Mal um den Degussitstab gewickelt. Zwischen den Wicklungen befinden sich die Thermoelemente, die aufgrund der hohen Wärmeleitfähigkeit von Silber $(407$ W/m K) in gutem Wärmekontakt zur Probe stehen. Die Silberfolie wird mit einer Schlauchschelle am Degussitrohr befestigt. Die Schlauchschelle sollte nicht höher als der untere Mantelheizleiter angebracht werden, um unnötigen Untergrund in

\footnotetext{
${ }^{4}$ Bei einem Kristall von $5 \times 7 \times 11 \mathrm{~mm}^{3}$ ist dies ca. $50 \times 50 \mathrm{~mm}^{2}$.
} 


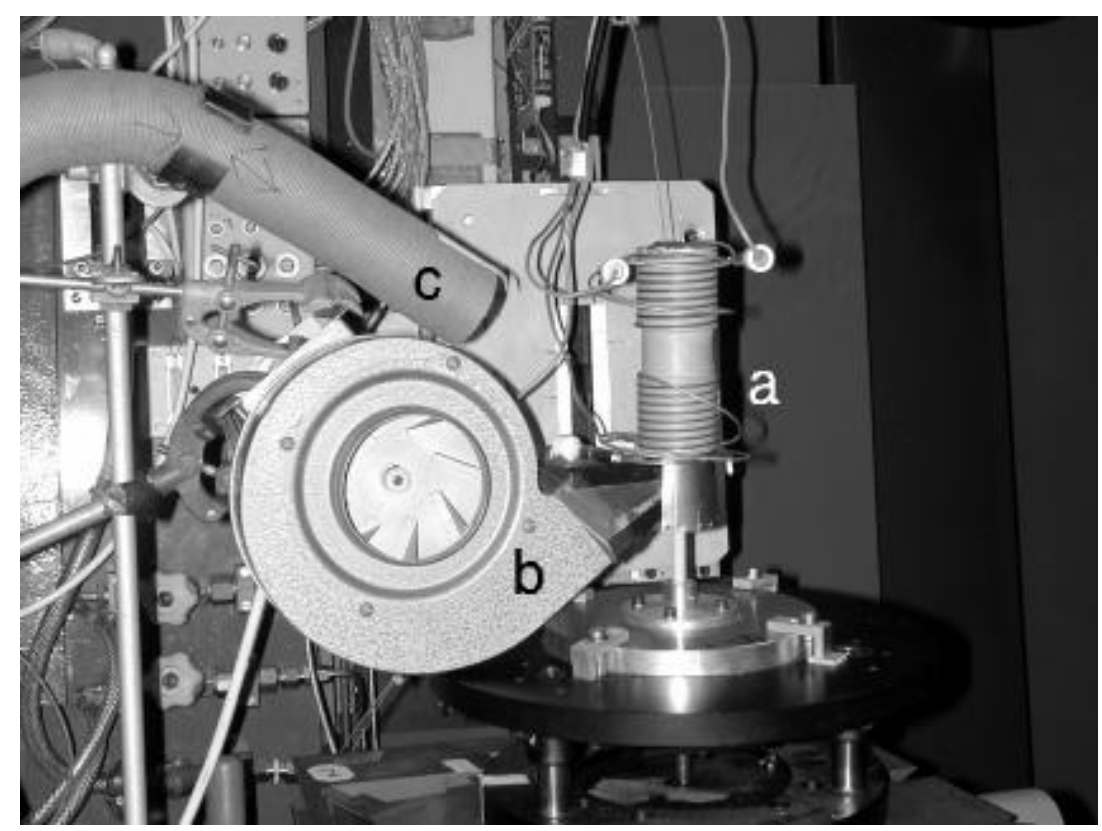

Abbildung 3.8: Der Probenaufbau am UNIDAS mit dem optimierten Neutronenofen

den Neutronenstreuexperimenten zu vermeiden. Dann wird die Probe von oben in die Hülse aus Silberfolie gesteckt. Die ca. 5-10 mm überstehende Folie wird vom oberen Ende umgeschlagen, bis der Kristall fest eingeschlossen ist. Um ein Lösen zu verhindern, werden die umgeschlagenen Kanten jeweils mit einer Zange zusammengepreßt. Die unterhalb der Schlauchschelle überstehende Silberfolie wird senkrecht geschnitten und aufgefächert. Neben der Fixierung des Probenkristalls verteilt die Silberfolie zusätzlich die Wärme über die gesamte Kristalloberfläche. Dies verhindert zu große Temperaturgradienten an der Probenoberfläche, die — wie Vorversuche ergaben — die Probenkristalle platzen lassen. Vor dem Experiment muß die Probe mehrmals aufgeheizt und anschließend abgekühlt werden, da es sonst durch die Silberfolie zu Dejustierungen kommen kann. Mit dieser Probenhalterung änderte sich die Mosaizität der Kristalle selbst dann nicht, wenn Risse in den Kristallen während eines Experimentes auftraten, da die Bruchstuicke stets sicher fixiert waren.

Die aktive Kühlung der Probe und des Ofens erfolgt mit Gebläsen, wie in Abbildung $3.8 \mathrm{zu}$ sehen ist. Die Raumluft wird durch den Anblasstutzen von unten (b) in den Ofen (a) eingeblasen, um die Probe abzukühlen. Der Ofen (a) selbst wird von außen (c) angeblasen. Um z.B. eine Probe von $5 \times 7 \times 11 \mathrm{~mm}^{3}$ mit $c_{p} \approx 650 \mathrm{~J} / \mathrm{g} \mathrm{K} \mathrm{um} \Delta T=300{ }^{\circ} \mathrm{C}$ abzukühlen, muß eine Wärmemenge von ca. $288 \mathrm{~J}$ abgeführt werden. Bei einer Abkühlzeit von ca. $30 \mathrm{~s}$ entspricht dies einer Kühlleistung von 10 W. Die maximale Kühlrate, die erreicht werden kann, ist durch das Temperaturprofil der Probe selbst gegeben. Sie kann mittels Berechnungen für einen unendlich langen Stab auf eine Größenordnung 
von ca. $30{ }^{\circ} \mathrm{C} / \mathrm{s}$ bei der oben genannten Probengröße abgeschätzt werden. Diese wird experimentell nicht zu erreichen sein, da zur Probe noch die Wärmekapazität des Ofens hinzukommt. Die beste experimentell erreichte Kühlleistung lag bei $7{ }^{\circ} \mathrm{C} / \mathrm{s}$, die aber für die kinetischen Experimente ausreichend ist.

Die Temperaturmessung erfolgte mit anfänglich drei $\mathrm{NiCr}$ - $\mathrm{Ni}$-Mantelthermoelementen der Firma Thermocoax oder glasfaserummantelten $\mathrm{NiCr}$ - $\mathrm{Ni}$-Thermoelementen der Firma Heraeus. Die Temperaturdifferenz der Mantelthermoelemente untereinander lag sowohl beim Abkühlen als auch bei statischen Bedingungen unabhängig von der Position an der Probe innerhalb der Toleranzen der Thermoelemente $( \pm 3 \%)$. Nachdem feststand, daß in allen Experimenten keine Temperaturgradienten an der Probenoberfläche auftraten, wurde die Probentemperatur nur noch mit einem Thermoelement gemessen.

\subsection{Die Zeitauflösung}

Viele kinetische Prozesse können durch zeitliche Änderung von Meßgrößen wie z.B. Schwingungsfrequenzen, Leitfähigkeiten oder Längen experimentell verfolgt werden. Dabei ist es notwendig, daß die Meßgröße in zeitlichen Abständen bestimmt wird, die der Kinetik angepaßt sind. Dieser Abstand wird im folgenden als Zeitkanalbreite $\Delta t$ bezeichnet. Aber gerade bei kleinen Zeitkanalbreiten reicht oftmals die Meßgenauigkeit für die Änderung der Meßgröße innerhalb eines Zeitkanals nicht aus. In laserspektroskopischen Experimenten ist es z.B. dann möglich, die Qualität des Meßsignals durch eine Erhöhung der Primärintensität zu verbessern. Bei Neutronenstreuexperimenten kann jedoch die Leistung nicht ohne weiteres erhöht werden ${ }^{5}$.

Eine Möglichkeit, kleine Zeitkanalbreiten — nicht nur — für die Neutronenstreuung zugänglich zu machen, ist die stroboskopische Meßtechnik [Eckold 1990, Eckold 1991]. Diese Meßtechnik kann bei allen reversiblen Prozessen eingesetzt werden, bei denen die Probe nach der spontanen Änderung eines externen Parameters, wie z.B. Temperatur, Druck oder elektrisches Feld, wieder ins Gleichgewicht relaxiert. Spontan bedeutet dabei, daß die Relaxationszeit der Probe viel größer sein muß als der Zeitraum, in dem der externe Parameter geändert wird. Diese Meßtechnik soll an dem folgenden Beispiel erklärt werden:

In einem statischen Experiment wird ein Phononenspektrum im Frequenzbereich $v_{0}$ bis $v_{1}$ mit einer Schrittweite $\Delta v$ gemessen werden. Es werden also $N=v_{1}-v_{0} / \Delta v$ Punkte gemessen. Die Zählstatistik für die Meßpunkte wird über eine entsprechende Monitorvorwahl eingestellt. Ist die Monitorvorwahl für einen Punkt erreicht, wird der nächste Punkt gemessen, bis das Spektrum vollständig ist. In einem kinetischen Experiment soll

${ }^{5}$ Die thermische Reaktorleistung hat sich seit dem ersten Forschungsreaktor in Brookhaven (USA) mit $20 \mathrm{MW}$ (1958) auf $58 \mathrm{MW}$ am Institut Laue-Langevin (F) nur verdreifacht. Die Weiterentwicklungen von Neutronenleitern und Experimentiergeräten haben die entscheidenden Beiträge zur Erhöhung des Neutronenflusses am Probenort geliefert. 
das gleiche Spektrum während der Relaxation in Abhängigkeit von der Zeit über einen Zeitraum, die Periodendauer $T$, gemessen werden. Die Zeitkanalbreite der Spektren soll dabei $\Delta t$ betragen, und die Messung hat demzufolge $n=T / \Delta t$ Zeitkanäle. Für diese Messung wird der erste Meßpunkt angefahren, die Probe in den Nichtgleichgewichtszustand gebracht, und die Meßwerterfassung gestartet. Es wird für jeden Zeitkanal des Meßpunktes die Zählrate gespeichert. Ist die Zählstatistik in den Zeitkanälen nicht ausreichend, wird die Probe wieder in den Nichtgleichgewichtszustand gebracht und die Messung wiederholt. Die Zählraten dieser zweiten Messung werden dann zu den Zählraten der ersten Messung aufaddiert. Dieser Vorgang wird so lange wiederholt, bis die Statistik ausreichend ist. Die Anzahl dieser Wiederholungen ist die Periodenzahl $P$. Dann wird bei den verbleibenden Meßpunkten genauso verfahren. Grundlegende Voraussetzung für die stroboskopische Meßttechnik ist aber, daß die Probe in jeder Periode zu jedem Zeitpunkt den gleichen Zustand durchläuft.

Im Falle von Silber-Natriumchlorid wird eine homogene Probe aus dem Einphasengebiet in die Mischungslücke abgeschreckt, vgl. Abb. 1.1, und die Probe entmischt. Wird die Probe erneut auf Temperaturen oberhalb der Binodalen aufgeheizt, bildet sich von neuem eine Phase mit der mittleren Zusammensetzung. Der Ausgangszustand ist wieder erreicht. Der Entmischungsprozeß ist reversibel und kann durch die Temperatur gesteuert werden. Diese thermodynamische Betrachtung reicht jedoch nicht für die Kinetik der Entmischung aus, da sie keinerlei Aussagen über die Zeiträume der Zustandsänderungen macht. Aus vorangegangenen elastischen Neutronenstreuexperimenten [Caspary 1998, Caspary u. a. 2001] ist bekannt, daß die frühen Stadien der Entmischung auf einer Zeitskala von $10^{2} \mathrm{~s}$ ablaufen, und daß eine derartig entmischte Probe mit $x_{0}=0.5$ durch Homogenisieren über mindestens 5 min bei $400{ }^{\circ} \mathrm{C}$ wieder in den Ausgangszustand ${ }^{6}$ überführt werden kann.

Abbildung 3.9 zeigt ein Spektrum von Kristall III bei $\mathbf{Q}=(20.250)$ und $400{ }^{\circ} \mathrm{C}$ nach Homogenisierungszeiten von 5 min bzw. $60 \mathrm{~min}$. Der Kristall wurde zuvor bei $100{ }^{\circ} \mathrm{C}$ ausgelagert. Zwischen den beiden Spektren sind keine signifikanten Unterschiede festzustellen, die auf einen Einfluß der unterschiedlichen Homogenisierungszeiten schließen lassen. Analoge Experimente mit den Kristallen anderer Zusammensetzung führen zu gleichen Ergebnissen. Hinzu kommt, daß in den kinetischen Experimenten die Homogenisierung zeitaufgelöst verfolgt wurde. Nach der Homogenisierungszeit von 5 min ist keine signifikante zeitliche Änderung der Spektren mehr zu beobachten. Es kann ebenfalls mit der inelastischen Neutronenstreuung festgestellt werden, daß eine bis zu 20 min bei $T \geq 100{ }^{\circ} \mathrm{C}$ entmischte Probe nach 5 min bei $400{ }^{\circ} \mathrm{C}$ sich wieder im homogenen Zustand befindet. Es ist also möglich, an $\mathrm{Ag}_{x} \mathrm{Na}_{1-x} \mathrm{Cl}$-Einkristallen stroboskopische Experimente durchzuführen.

Das einzige Neutronendreiachsspektrometer, in dessen Steuerung die stroboskopi-

${ }^{6}$ Der Begriff ,,im gleichen Zustand“ faßt alle Zustände zusammen, die mit dem jeweiligen Experiment nicht unterscheidbar sind. 


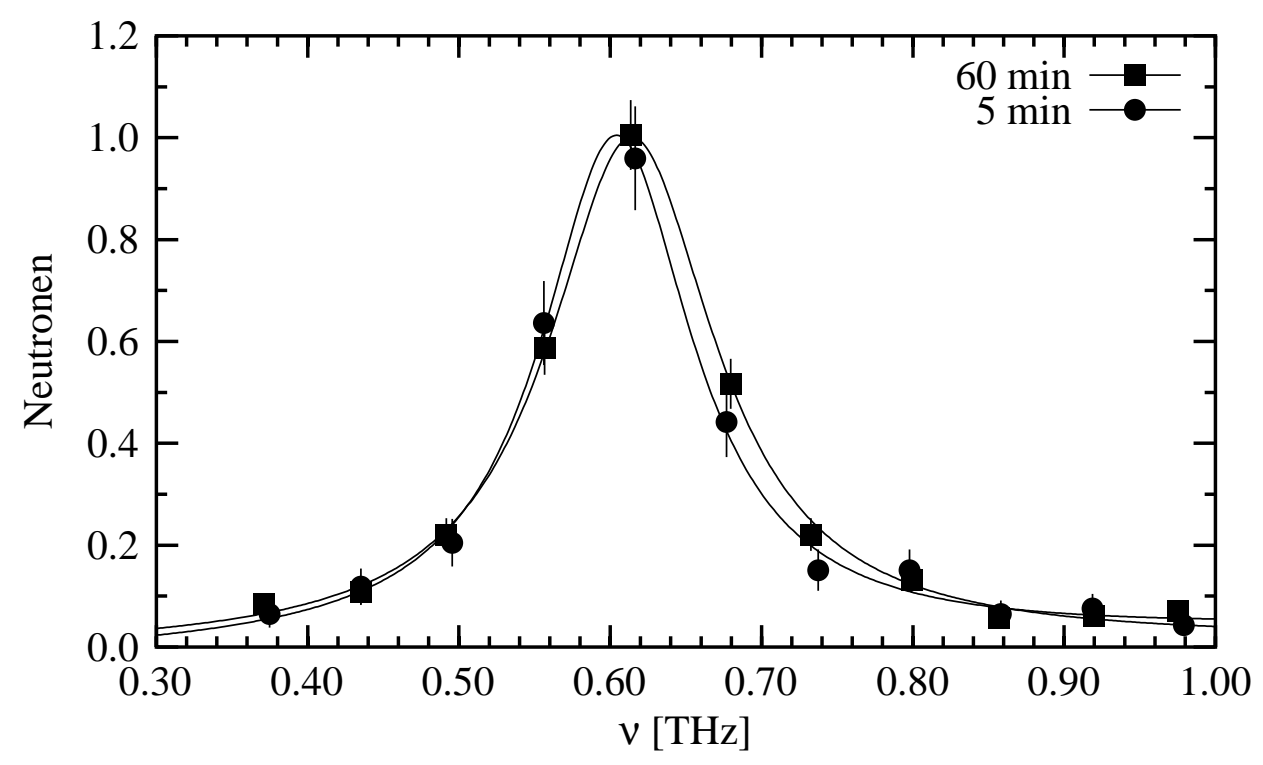

Abbildung 3.9: Zwei Spektren nach unterschiedlichen Homogenisierungszeiten bei $400{ }^{\circ} \mathrm{C}$ bei $Q=\left(\begin{array}{lll}2 & 0.25 & 0\end{array}\right)$

sche Meßtechnik implementiert ist, ist das UnIDAS. Um stroboskopische Experimente an anderen Neutronenquellen durchführen zu können, wurde eine transportable Zeitauflösung auf der Basis einer „multichannel scaler“ (MCS) Karte der Firma EG\&G Ortec entwickelt. Die Hauptaufgabe war die Synchronisation von Zählkarte, Spektrometer und Probenumgebung. In Abbildung 3.10 sind der schematische Versuchsaufbau und die Steuersignale aller Komponenten dargestellt. Um diese drei Komponenten zu synchronisieren, wird ein Funktionsgenerator verwendet, der zu jedem Periodenbeginn einen Synchronisationspuls gibt und so das Experiment taktet. Der Signalausgang des Funktionsgenerators wird so programmiert, daß er für die Dauer des Auslagerns von 0 bis $t_{1}$ ein „high“- und für den Zeitraum des Homogenisierens von $t_{1}$ bis $T$ ein „low“Signal am Signalausgang liefert. Mit diesem Signal wird der Sollwert am Temperaturregler umgeschaltet. Mit dem Auslagern der Probe beginnt die Datenerfassung an der MSC-Karte, die mit dem Synchropuls des Funktionsgenerators getriggert wird. Zu Beginn der Datenerfassung muß das Spektrometer in der richtigen Konfiguration für den Meßpunkt sein. Ist das Spektrometer in der richtigen Konfiguration, liefert es ein TTLSignal, das ein „Meßbereit“ signalisiert. Durch eine UND-Verknüpfung des Synchronisationspulses mit diesem „Meßbereit“-Signal des Spektrometers wird verhindert, daß die MSC-Karte gestartet wird, wenn das Spektrometer aus einem unbestimmten Grund nicht meßbereit sein sollte. Als Meßsignal wird der Detektorausgang des Spektrometers auf den Zähleingang der Karte gegeben.

Nach $P$ Perioden muß das Spektrometer für den nächsten Meßpunkt positioniert wer- 

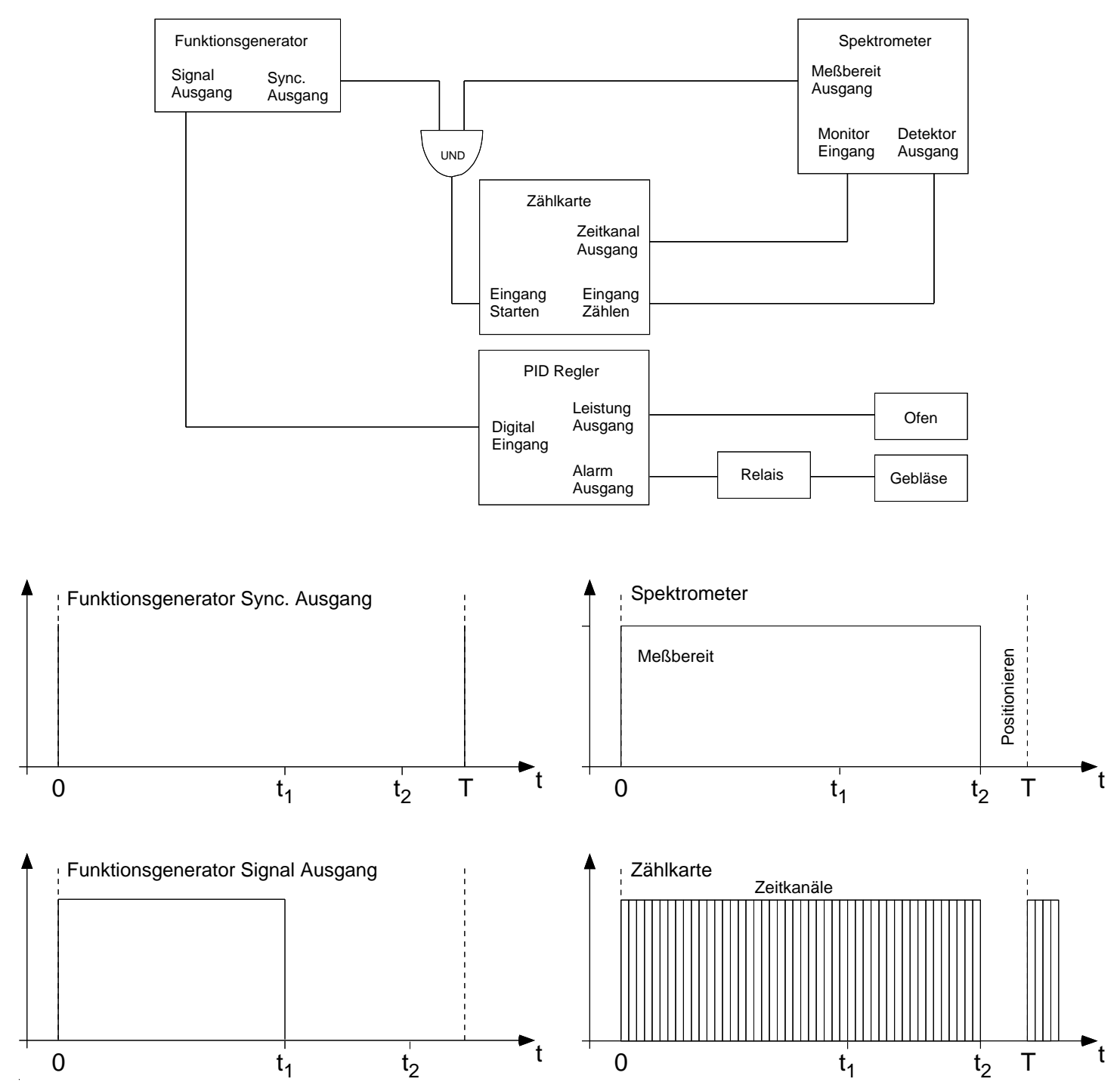

Abbildung 3.10: Die Pulsdiagramme der in den zeitaufgelösten Experimenten verwendeten Elektronik und deren Blockbild

den. Dieser Zeitpunkt des Positionierbeginns wird durch die Monitorvorwahl am Spektrometer festgelegt. Die MCS-Karte gibt am Ende eines jeden Zeitkanals einen Puls. Dieser Puls des „Advanced Channel Out“ Ausgangs wird als Monitorpuls am Spektrometer verwendet. Die Monitorvorwahl ${ }^{7}$ ist dann $P \cdot n \cdot N$. Die Positionierung sollte vor dem Beginn der nächsten Periode zwischen $t_{2}$ und $T$ abgeschlossen sein. Die Zeit, die dem Spektrometer zur Verfügung steht, ist $T-(P \cdot n \cdot N \cdot \Delta t)$. Aus technischen Gründen kann es vorkommen, daß das Positionieren des Spektrometers länger dauert als die Zeit

\footnotetext{
${ }^{7}$ Dabei ist die Besonderheit des Monitors am IN-12 zu berücksichtigen: Er kann z.Z. nur auf zwei Dezimalstellen zählen, z.B. $12,120,1200$, usw. Bei z.B. $T=1200 \mathrm{~s}, \Delta t=10 \mathrm{~s}$ und $P=3$ ist die MCS-Karte auf 110 Zeitkanäle zu programmieren und den Monitor auf 330 vorzuwählen.
} 
zwischen $t_{2}$ und $T$. Diese mögliche Fehlerquelle wird durch die UND-Verknüpfung mit dem „Meßbereit“-Signal abgefangen, und die Messung wird mit der nächsten Periode korrekt fortgesetzt, wobei allerdings ein kompletter Meßzyklus verloren geht.

Um die Probe aktiv kühlen zu können, werden über zwei separate Alarmausgänge des PID-Reglers zwei Gebläse mittels Festkörperrelais ein- und ausgeschaltet. Die Alarmausgänge sind so konfiguriert, daß die Gebläse bei Sollwertabweichungen von $10{ }^{\circ} \mathrm{C}$ für die innere und $3{ }^{\circ} \mathrm{C}$ für die äußere Kühlung eingeschaltet sind. Die Regelung des Ofens erfolgte mit einem Proportionalanteil von $9 \%$, einem Differenzialanteil von $11.7 \%$ und einer Integrationszeit von $70 \mathrm{~s}$.

Neben der MCS-Karte wurden als Komponenten ein Funktionsgenerator, AFUG der Fa. Rohde \& Schwarz oder PM 5139 der Fa. Fluke, und ein Eurotherm PID-Temperaturregler 900 EPC verwendet. Die UND-Verknüpfung wurden von der Elektronikwerkstatt des Instituts für Physikalische Chemie der Universität Göttingen (IPC) gebaut. 


\section{Ergebnisse der statischen Experimente}

Im Umfeld der Vorbereitung und Durchführung der kinetischen Experimente zur Untersuchung der Entmischungskinetik wurde eine Vielzahl von zusätzlichen Meßdaten gewonnen. In diesem Kapitel werden die Ergebnisse dieser statischen Experimente präsentiert. Die statischen Experimente wurden bei Temperaturen oberhalb der Binodalen im homogenen Bereich des Phasendiagramms und bei Raumtemperatur durchgeführt. Es wird sich zeigen, daß die Ergebnissen aus den statischen Experimenten für die Interpretation der kinetischen Experimente notwendig sind.

\subsection{Dispersionskurven im homogenen Zustand}

Um die Dispersionen im homogenen Bereich des Phasendiagramms zu messen, wurden die Proben so lange homogenisiert, bis sich die Spektren zeitlich nicht mehr änderten. Abbildung 4.1 zeigt ein Spektrum bei $400{ }^{\circ} \mathrm{C}$ und einer mittleren Zusammensetzung von $x_{0}=0.41$. Dieses Spektrum ist repräsentativ für die in der homogenen Phase gemessenen Spektren. Die durchgezogene Linie in Abbildung 4.1 ist eine an die Meßpunkte angepaßte Lorenzfunktion ${ }^{1}$

$$
I(v)=I_{\max } \frac{(\Delta v)^{2}}{4\left(v-v_{\max }\right)^{2}+(\Delta v)^{2}} .
$$

Das Phonon liegt hier bei $v_{\max }=-0.498 \mathrm{THz}$ und hat eine Halbwertsbreite von $\Delta v=$ $0.101 \mathrm{THz}$, die der Geräteauflösung ${ }^{2} \Delta_{v}$ entspricht. Beim Vergleich der Frequenzlage mit den extrapolierten Frequenzen der reinen Substanzen liegt $v_{\text {max }}$ zwischen $\mathrm{AgCl}$ und $\mathrm{NaCl}$, wie es zunächst zu erwarten ist. Es fällt jedoch auf, daß das Phonon dichter an der Frequenz von reinem Silberchlorid liegt, obwohl im Kristall der Natriumchloridanteil größer als der $\mathrm{AgCl}$-Anteil ist. Es wurden im homogenen Zustand die akustischen Dispersionszweige für $x_{0}=0.23$ und $x_{0}=0.26$ und einige Meßpunkte für $x_{0}=0.41$ gemessen. Abbildung 4.2 zeigt die Dispersionen für eine mittlere Zusammensetzung

\footnotetext{
${ }^{1}$ Zur Beschreibung von Spektren in denen ausschließlich $v<0$ oder $v>0$ gemessen wurde, können schwach gedämpfte Phononen nach Gleichung (1.35) mit nur einer Lorenzfunktion beschrieben werden.

${ }^{2}$ Die Geräteauflösung ist abhängig von der Konfiguration des Spektrometers und den Probeneigenschaften wie z.B. Gitterkonstante und Mosaizität und der jeweiligen Dispersionskurve. Zur Berechnung der Auflösung mit dem Programmpaket PkFit [A.Bouvet und A.Filhol 1995, A.Bouvet u. a. 1997] wurde als Dispersion eine lineare Abhängigkeit mit der Steigung $v_{\max } / \xi$ mit $\xi=0.2$ verwendet.
} 
von 0.26. Wie das gezeigte Phonon bei $x_{0}=0.41$ lassen sich die Dispersionskurven der Mischkristalle gut zwischen den Dispersionskurven der reinen Substanzen einordnen. Wie aufgrund des Stoffmengenanteils zu erwarten, liegen die Dispersionen dichter an den Dispersionskurven des $\mathrm{NaCl}$.

Werden die Dispersionskurven für $q \rightarrow 0$ extrapoliert, können die Schallgeschwindigkeiten bestimmt werden. Ist zusätzlich die Massendichte bekannt, können die elastischen Konstanten berechnet werden. Für die Massendichte ist

$$
\rho=\frac{4\left[x_{0} \cdot m_{A g}+\left(1-x_{0}\right) \cdot m_{N a}+m_{C l}\right]}{a^{3}}
$$

angesetzt worden. Als Gitterkonstante werden die experimentell bestimmten Werte verwendet. Die Ergebnisse sind in Tabelle 4.1 zusammengefaßt. Neben den Ergebnissen für die elastischen Konstanten bzw. deren Kombination sind zum Vergleich die extrapolierten Werte für die elastischen Konstanten, wie man sie aus einer Linearkombination

$$
c_{i j}(x)=x_{0} \cdot c_{i j}^{A g C l}\left(1+\Delta T \cdot T_{c_{i j}}^{A g C l}\right)+\left(1-x_{0}\right) \cdot c_{i j}^{N a C l}\left(1+\Delta T \cdot T_{c_{i j}}^{N a C l}\right)
$$

aus den temperaturkorrigierten reinen Substanzen erwartet, mit aufgeführt.

Aus den Ergebnissen in Tabelle 4.1 können die elastischen Konstanten für $x_{0}=0.26$ mehrfach berechnet werden, weil das System überbestimmt ist. Für $c_{11}$ ergeben sich

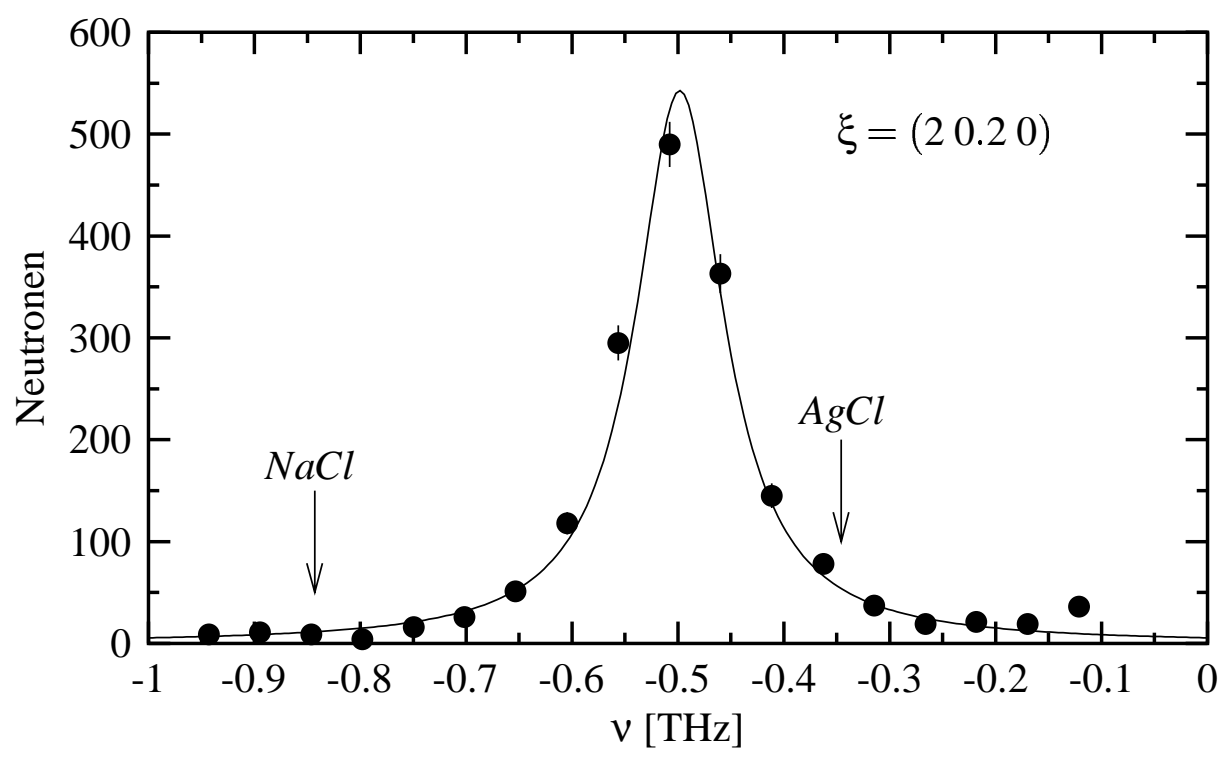

Abbildung 4.1: Ein für alle TA Spektren in der homogenen Phase repräsentatives Beispielspektrum bei $400{ }^{\circ} \mathrm{C}$ und $x_{0}=0.41$. Das Spektrum ist mit $\Delta E<0$ gemessen, so daß die gemessene Intensität gegen negative Frequenz aufgetragen wird. Die beiden Pfeile markieren die für die reinen Substanzen nach Gleichung (2.8) extrapolierten Frequenzen. 

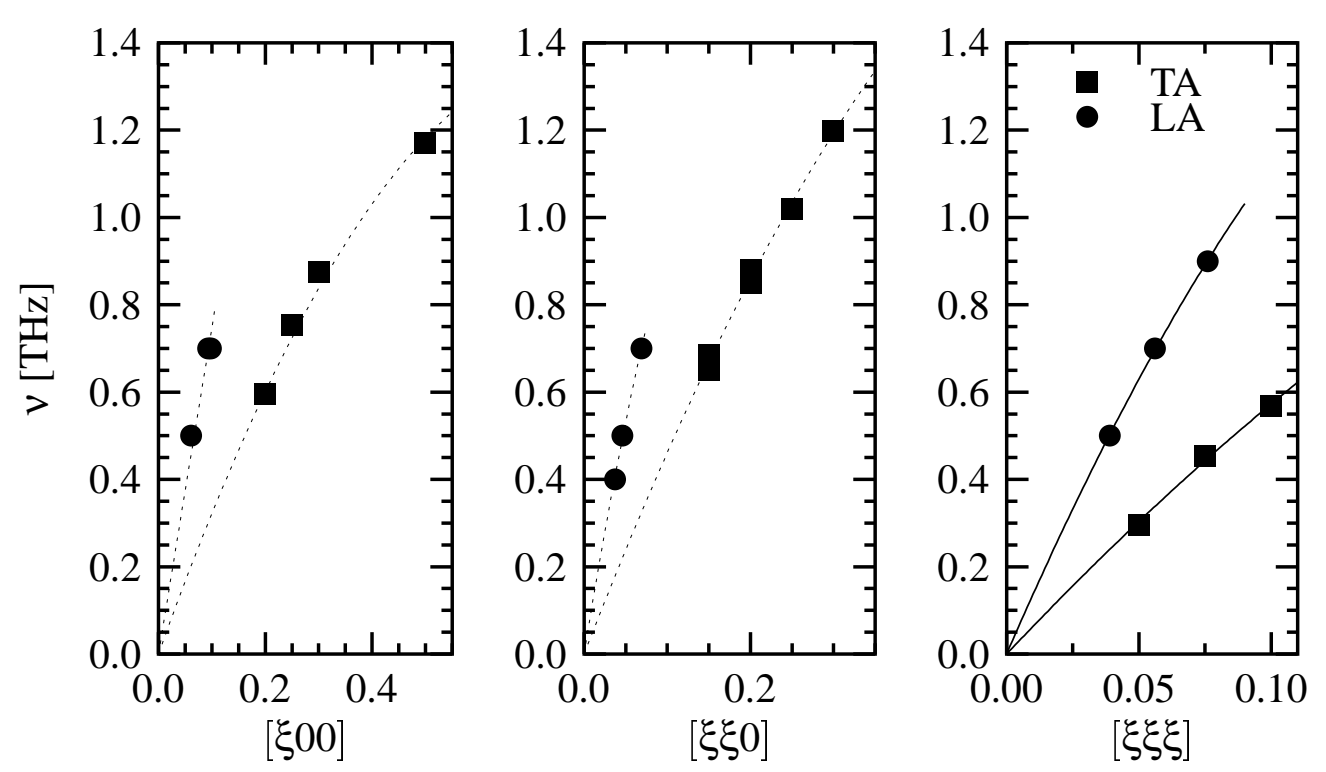

Abbildung 4.2: Die Dispersionskurven des TA und LA Phonons in [ [ $\left.\begin{array}{lll}1 & 0 & 0\end{array}\right]$ und $\left[\begin{array}{lll}1 & 1 & 1\end{array}\right]$ und $\left[\begin{array}{lll}1 & 1 & 0\end{array}\right]$ mit $x_{0}=0.26$ bei $333{ }^{\circ} \mathrm{C}$

Z.B.

$$
\begin{aligned}
c_{11} & =63 \mathrm{GPa} \\
& =58 \mathrm{GPa}=c_{B}-c_{A}+\frac{1}{2}\left(c_{D}-c_{B}-c_{A}\right) \\
& =51 \mathrm{GPa}=c_{E}-3 c_{A}+c_{B}-c_{D} \\
& =65 \mathrm{GPa}=2 c_{D}-c_{E},
\end{aligned}
$$

oder für $c_{12}=35,30,25$ und $24 \mathrm{GPa}$. Es zeigt sich, daß die Ergebnisse in sich konsistent sind. Für die $26 \%$-Probe steht $c_{44}$ in befriedigender Übereinstimmung mit der Prognose. In der $23 \%$-Probe unterscheiden sich die beiden Ergebnisse für $c_{44}$ schon erheblich. Noch schlechter ist die Übereinstimmung von Vorhersage und Experiment für die Kombinationen mit $c_{11}$ und $c_{12}$; der extrapolierte Wert für $c_{12}$ ist $19 \mathrm{GPa}$. Diese großen Abweichungen sind in erster Linie auf die fehlenden Meßdaten in der Nähe des $\Gamma$-Punktes zurückzuführen. Es kann zwar eine Dispersion aus den Meßdaten konstruiert werden, dennoch ist es nicht gesichert, daß die wahre Dispersion in der Nähe des $\Gamma$-Punktes den gleichen Verlauf hat. Es ist zudem bekannt, daß die für Alkalihalogenide bei Raumtemperatur mit inelastischer Neutronenstreuung bestimmten elastischen Konstanten um bis zu 10 \% über den Werten liegen, die aus Ultraschalluntersuchungen erhalten werden [Cowley 1967]. Hinzu kommen die Fehler aus der Berechnung der Massendichte mit der Gitterkonstanten und den mittleren Konzentrationen. So kann der Fehler für die elastischen Konstanten bzw. deren Kombination mit $\Delta c / c \approx 15 \%$ für $c_{44}$ 
Tabelle 4.1: Die Schallgeschwindigkeiten und elastischen Konstanten der homogenen Phasen. Zum Vergleich stehen in Klammern die mittels Linearkombination (4.3) extrapolierten Werte für die elastischen Konstanten bzw. deren Kombination.

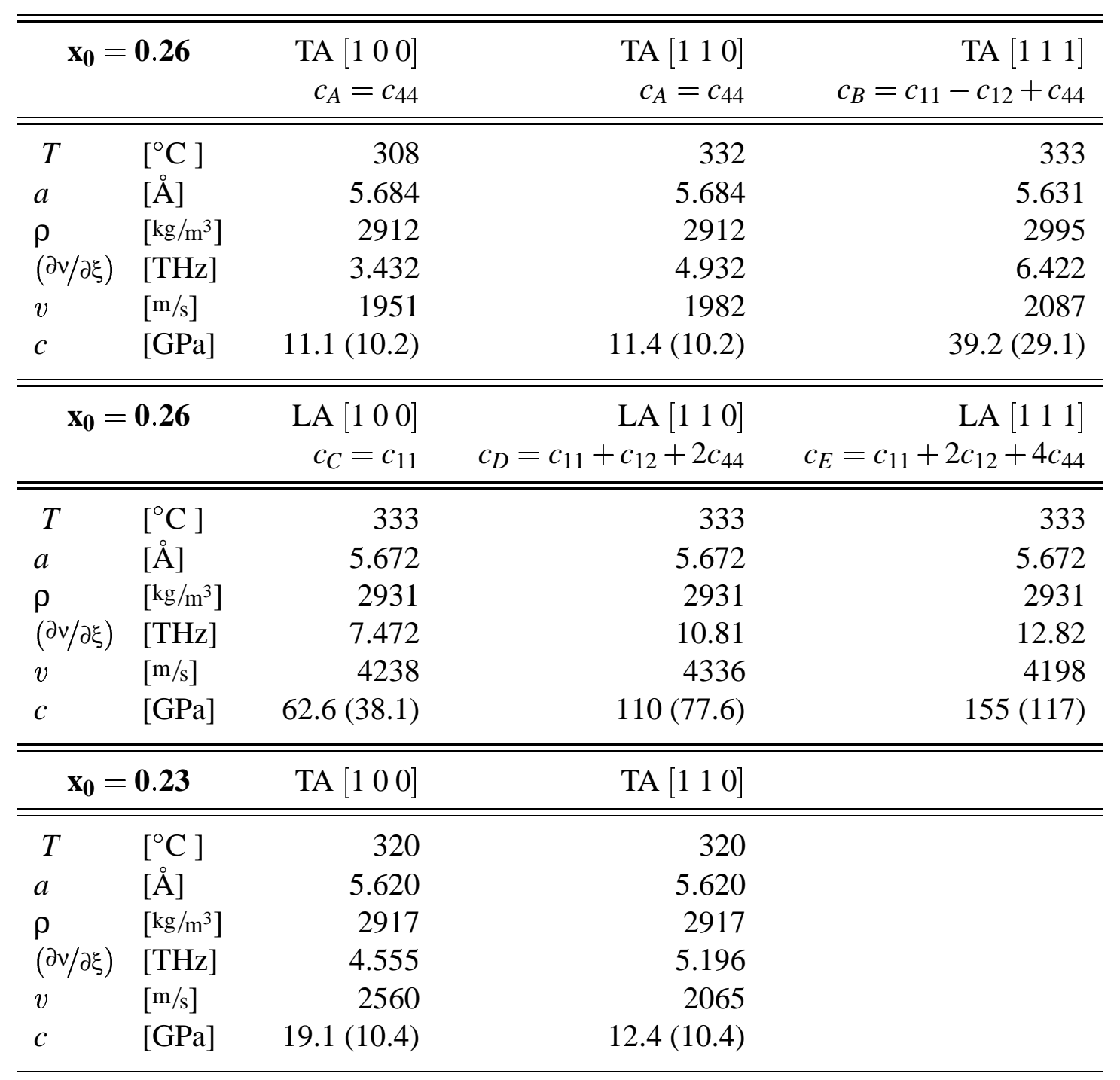

und $35 \%$ für die Kombination aus $c_{11}$ und $c_{12}$ abgeschätzt werden. Es ist weiterhin die Annahme, daß die Temperaturkoeffizienten der elastischen Konstanten über einen Temperaturbereich von ca. $300 \mathrm{~K}$ konstant bleiben, sehr vorsichtig zu bewerten. Es war aber auch nicht das Ziel dieser Experimente, die Dispersionskurven für $q \rightarrow 0$ und die elastischen Konstanten der Mischkristalle genau zu bestimmen. Dennoch zeigen die Ergebnisse in sich eine überraschend gute Konsistenz. 


\subsection{Das Frequenz-Konzentrations Diagramm}

Während der kinetischen Experimente werden keine Dispersionskurven gemessen, sondern nur Spekten bei einem konstanten Wellenvektor. Für die Auswertung der kinetischen Experimente ist es folglich evident, die Abhängigkeit der Frequenz eines Phonons von der Zusammensetzung $v(x)$ genau zu kennen. Aus den vorliegenden Dispersi-

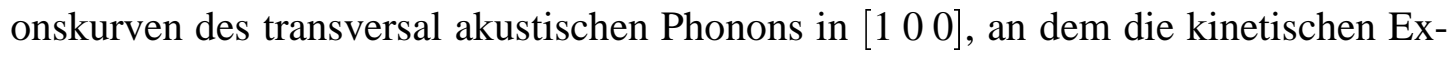
perimente erfolgen sollen, kann ein Diagramm $v$ versus $x$ erstellt werden. Die Dispersionen in der homogenen Phase wurden bei unterschiedlichen Temperaturen gemessen, so daß $400{ }^{\circ} \mathrm{C}$ als Referenztemperatur gewählt wird. Da nur für drei Zusammensetzungen die Dispersionen experimentell zugänglich waren, wurden aus bekannten weiteren Wertepaaren $[x, v(x, T)]$ die Werte für $[x, v(x, T+\Delta T)]$ nach Gleichung (2.8) extrapoliert. Zwei Punkte wurden aus den reinen Substanzen extrapoliert. Die Frequenzen der reinen Komponenten liegen bei $400{ }^{\circ} \mathrm{C}$ und $\xi=0.3$ unter Berücksichtigung der Temperaturkoeffizienten aus Tabelle 2.1 bei $v_{\mathrm{NaCl}} \approx 1.2 \mathrm{THz}$ und $\mathrm{v}_{\mathrm{AgCl}} \approx 0.55 \mathrm{THz}$. Analog hierzu konnten aus den Dispersionskurven der Proben nach langen Auslagerungszei$\operatorname{ten}^{3} v_{x=0.05} \approx 1.1 \mathrm{THz}$ und $v_{x=0.95} \approx 0.52 \mathrm{THz}$ extrapoliert werden. Für den Temperaturkoeffizienten der gemischten, homogenen Phase wurde die Linearkombination $T_{c_{44}}=x \cdot T_{c_{44}}^{\mathrm{AgCl}}+(1-x) \cdot T_{c_{44}}^{\mathrm{NaCl}}$ und $\alpha_{c_{44}}=x \cdot \alpha_{c_{44}}^{A g C l}+(1-x) \cdot \alpha_{c_{44}}^{\mathrm{NaCl}}$ verwendet. Bei Temperaturänderungen von $\Delta T=100{ }^{\circ} \mathrm{C}$ betragen die relativen Änderungen von $\Delta v / \Delta \xi$ nach Gleichung (2.8) nicht mehr als $3 \%$. Aus diesem Grund kann auf eine Korrektur der experimentell bei unterschiedlichen Homogenisierungstemperaturen bestimmten Frequenzen verzichtet werden.

Das Ergebnis für $\xi=0.3$ ist in Abbildung 4.3 gezeigt. Die $N a$-reichen Zusammensetzungen haben deutlich höhere Frequenzen, als die $A g$-reichen, wie es anhand der reinen Komponenten schon zu erwarten ist. Es ist aber deutlich zu erkennen, daß es sich um keinen linearen Zusammenhang handelt. Im Bereich bis zu $50 \%$ Silberchloridanteil ist die Änderung der Frequenz deutlich größer als im Bereich mit höheren Anteilen. Dies erklärt, daß in Abbildung 4.1 das Phonon dichter an der Frequenz von reinem $\mathrm{AgCl}$ liegt. Vom prinzipiellen Verlauf her ist die $v(x)$-Kurve der Soliduslinie im Phasendiagramm sehr ähnlich, was anschaulich erklärt werden kann: Wird ein Festkörper mit seiner Schmelze verglichen, so ist es eine Erfahrungstatsache, daß die Flüssigkeit sich nicht einer Scherung widersetzt. Für einen Festkörper kann so die Temperaturabhängigkeit des Schermoduls durch den Ansatz [Ubbelohe 1965] $G=G^{\circ}(x)\left(1-T / T_{\text {Schmelz }}\right)^{2}$ ausgedrückt werden. Die Scherung wird durch die Komponente $\varepsilon_{6}$ des Dehnungstensors beschrieben, die proportional zur Scherspannung $\sigma_{6}$ und zu $1 / c_{44}$ ist: $\varepsilon_{6}=\sigma_{6} / c_{44}$. Es muß analog $c_{44}=c_{44}^{\circ}(x)\left(1-T / T_{\text {Schmelz }}\right)^{2}$ gelten. Mit diesem einfachen Ansatz ist der ähnliche Verlauf der Konzentrationsabhängigkeit der Frequenz des TA Phonons in $\left[\begin{array}{lll}1 & 0 & 0\end{array}\right]$ und der Schmelzkurve zu verstehen.

\footnotetext{
${ }^{3}$ siehe Abschnitt 4.4 auf Seite $67 \mathrm{ff}$
} 
Mikroskopisch ist die Abhängigkeit der Frequenz von der Zusammensetzung auf den Austausch von Natrium- gegen Silberatome zurückzuführen. Mit dem Austausch ändern sich die mittlere Masse des Kations und die Wechselwirkungpotentiale. Für ein

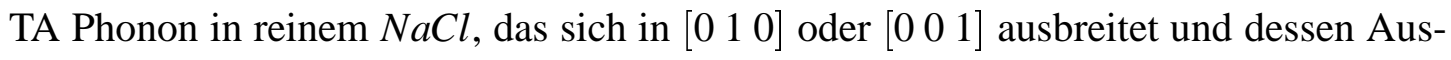
lenkung parallel zu $\left[\begin{array}{lll}1 & 0 & 0\end{array}\right]$ ist, ist der Eigenvektor für $q \rightarrow 0$

$$
\mathbf{e}=\frac{1}{\sqrt{m_{N a}+m_{C l}}}\left(\begin{array}{c}
\sqrt{m_{N a}} \\
0 \\
0 \\
\sqrt{m_{C l}} \\
0 \\
0
\end{array}\right),
$$

der als zweidimensionaler Vektor

$$
\mathbf{e}=\frac{1}{\sqrt{m_{N a}+m_{C l}}}\left(\begin{array}{l}
\sqrt{m_{N a}} \\
\sqrt{m_{C l}}
\end{array}\right)
$$

verkürzt dargestellt werden kann. Die Eigenwertgleichung (1.16) reduziert sich somit

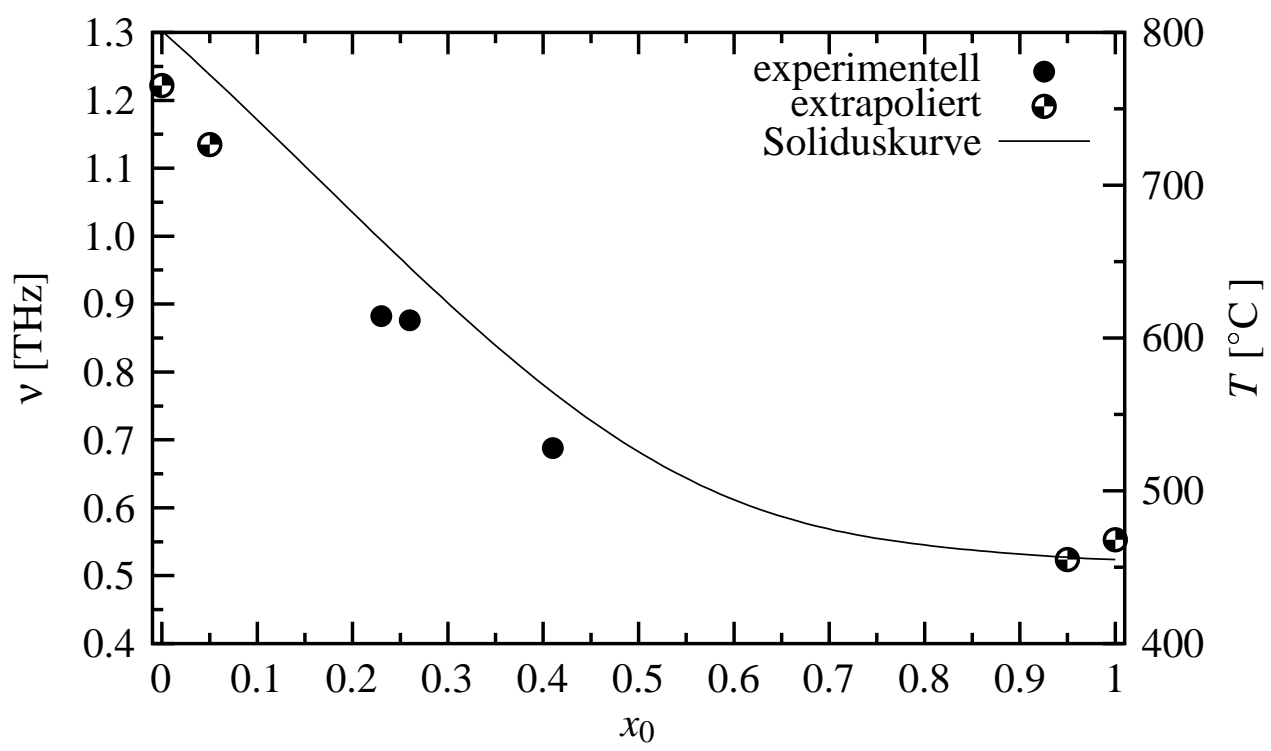

Abbildung 4.3: Die Abhängigkeit der Frequenz des TA Phonons bei $\xi=0.3$ von der Zusammensetzung bei $400{ }^{\circ} \mathrm{C}$. Da nur für drei Zusammensetzungen experimentelle Werte vorliegen, sind aus den Spektren der reinen Phasen und der Spektren nach langen Auslagerungszeiten weitere Werte extrapoliert worden. Die Lage der Frequenzen skaliert mit der Schmelzkurve, die zum Vergleich mit aufgetragen ist. 
auf

$$
\begin{aligned}
\omega^{2} & =\mathbf{e}^{\mathrm{T}} \underline{\underline{D}} \mathbf{e} \\
& =\frac{1}{\sqrt{M}}\left(\sqrt{m_{N a}} \sqrt{m_{C l}}\right)\left(\begin{array}{cc}
D_{N a N a} & D_{N a C l} \\
D_{C l N a} & D_{C l C l}
\end{array}\right) \frac{1}{\sqrt{M}}\left(\begin{array}{c}
\sqrt{m_{N a}} \\
\sqrt{m_{C l}}
\end{array}\right) \\
& =\frac{1}{M}\left[m_{N a} D_{N a N a}+m_{C l} D_{C l C l}+\sqrt{m_{N a} m_{C l}}\left(D_{N a C l}+D_{C l N a}\right)\right]
\end{aligned}
$$

mit $M=m_{N a}+m_{C l}$. Die Elemente der Matrix sind

$$
\begin{aligned}
D_{\kappa \kappa^{\prime}} & =-\sum_{\ell^{\prime}} \frac{V_{\kappa \ell \kappa^{\prime} \ell^{\prime}}}{\sqrt{m_{\mathrm{K}} m_{\mathrm{K}^{\prime}}}} \mathrm{e}^{\imath q\left(r_{\ell^{\prime}}-r_{\ell}\right)} \\
& \approx-\sum_{\ell^{\prime}} \frac{V_{\kappa \ell \kappa^{\prime} \ell^{\prime}}}{\sqrt{m_{\mathrm{K}} m_{\mathrm{K}^{\prime}}}}\left[1+\frac{l q r}{1}-\frac{(q r)^{2}}{2}\right] .
\end{aligned}
$$

Wenn das Produkt in der Summe von Gleichung (4.5) ausmultipliziert wird, summieren sich die beiden ersten Terme zu Null auf. Der erste Term ist gleich Null, weil im Ruhezustand jedes Atom kräftefrei sein muß. Die Terme mit dem Faktor ( $v q r)$ summieren sich ebenfalls zu Null auf, weil es zu jedem Abstand $r$ den Abstand $-r$ gibt. So vereinfacht sich Gleichung (4.5) zu

$$
D_{\kappa \kappa^{\prime}}=\sum_{\ell^{\prime}} \frac{V_{\kappa \ell \kappa^{\prime} \ell^{\prime}}}{\sqrt{m_{\kappa} m_{\kappa^{\prime}}}} \frac{q^{2} r^{2}}{2} .
$$

Wird (4.6) in Gleichung (4.4) eingesetzt folgt, daß

$$
\begin{aligned}
& \omega^{2} M=\frac{q^{2}}{2}\left[\sum_{N a} V_{N a N a} r_{N a N a}^{2}+\sum_{C l} V_{C l C l} r_{C l C l}^{2}\right. \\
&\left.+\sum_{C l} V_{N a C l} r_{N a C l}^{2}+\sum_{N a} V_{C l N a} r_{C l N a}^{2}\right] .
\end{aligned}
$$

Diese Gleichung läßt sich mit $V_{\mathrm{NaCl}}=V_{\mathrm{ClNa}}$ und $r_{\mathrm{NaCl}}=r_{\mathrm{ClNa}}=r_{\mathrm{NaNa}}=r_{\mathrm{ClCl}}$ weiter vereinfachen:

$$
\begin{aligned}
v^{2}=\frac{\omega^{2}}{q^{2}} & =\frac{1}{2 M}\left[\sum_{N a} V_{N a N a} r_{N a N a}^{2}+\sum_{C l} V_{C l C l} r_{C l C l}^{2}+2 \cdot \sum_{C l} V_{N a C l} r_{N a C l}^{2}\right] \\
& =\frac{1}{M} \cdot \sum_{C l} V_{N a C l} r_{N a C l}^{2}+\frac{1}{2 M} r_{N a N a}^{2}\left[\sum_{N a} V_{N a N a}+\sum_{C l} V_{C l C l}\right] \\
& =\frac{c_{44}}{\rho}=\frac{c_{44} \cdot a^{3}}{4 M}
\end{aligned}
$$


Im Mischkristall wird sich das Coulomb-Potential in erster Näherung nicht mit der Zusammensetzung ändern, sondern liefert einen konstanten Beitrag. Die Frequenzänderung ist dann von den kurzreichweitigen Wechselwirkungen abhängig. Bei auschließlicher Betrachtung nächster Nachbar Wechselwirkungen folgt aus Gleichung (4.8)

$$
v^{2}=\frac{1}{M} \cdot V_{N a C l} \cdot\left(\frac{a}{2}\right)^{2}=c_{44} \frac{a^{3}}{4 M} \quad \Leftrightarrow \quad V_{N a C l}=a \cdot c_{44} .
$$

Das Wechselwirkungspotential $\mathrm{V}_{\mathrm{NaCl}}$ ist bei dieser Betrachtung das Produkt aus der elastischen Konstante $c_{44}$ und der Gitterkonstanten $a$.

In einem $\mathrm{AgCl}$ - $\mathrm{NaCl}$-Mischkristall sind die Wahrscheinlichkeiten für eine $\mathrm{Ag} \leftrightarrow \mathrm{Cl}$ bzw. $\mathrm{Na} \leftrightarrow \mathrm{Cl}$ Wechselwirkung $x$ bzw. $(1-x)$. Die Schallgeschwindigkeit im Mischkristall ist dann analog $\mathrm{zu}(4.9)$

$$
\begin{aligned}
v^{2} & =\frac{1}{M_{x}}\left[x \cdot V_{A g C l}+(1-x) \cdot V_{N a C l}\right]\left(\frac{a}{2}\right)^{2} \\
& =\frac{a^{3}}{4 M_{x}} \underbrace{\left[x \cdot c_{44}^{A g C l}+(1-x) \cdot c_{44}^{N a C l}\right]}_{c_{44}(x) \text { des Mischkristalls }}=\frac{c_{44}(x)}{\rho(x)} .
\end{aligned}
$$

Die elastische Konstante $c_{44}$ des Mischkristalls ergibt sich durch Linearkombination der reinen Substanzen. Die Frequenz im Bereich $\xi \rightarrow 0$ ist dann nach

$$
v=\xi \cdot \sqrt{\frac{a \cdot\left[x \cdot c_{44}^{A g C l}+(1-x) \cdot c_{44}^{N a C l}\right]}{4\left[x \cdot m_{A g}+(1-x) \cdot m_{N a}+m_{C l}\right]}} .
$$

$\mathrm{zu}$ berechnen.

In Abbildung 4.4 ist Gleichung (4.11) mit den Meßdaten aufgetragen. Die berechneten Werte liegen deutlich über den experimentell bestimmten Frequenzen. Eine Beschreibung der Abhängigkeit der Schallgeschwindigkeit durch die Linearkombination der elastischen Konstanten über den gesamten Konzentrationsbereich ist demzufolge nicht möglich. Die vernachlässigten Wechselwirkungen müssen zu einer Erniedrigung der Schallgeschwindigkeiten führen. Um Frequenzen über den ganzen Konzentrationsbereich berechnen zu können, wurde ein Polynom

$$
v(x)=A+B \cdot(x-1)^{2}+C \cdot(x-1)^{3}
$$

an die Daten angepaßt. Die erhaltenen Parameter sind $A=0.52 \pm 0.02 \mathrm{THz}, B=0.16 \pm$ $0.18 \mathrm{THz}$ und $C=-0.55 \pm 0.18 \mathrm{THz}$.

Die homogene Phase, aus der in den kinetischen Experimenten abgeschreckt wird, zeichnet sich durch ein definiertes Kristallgitter aus, in denen die Kationenplätze sta- 


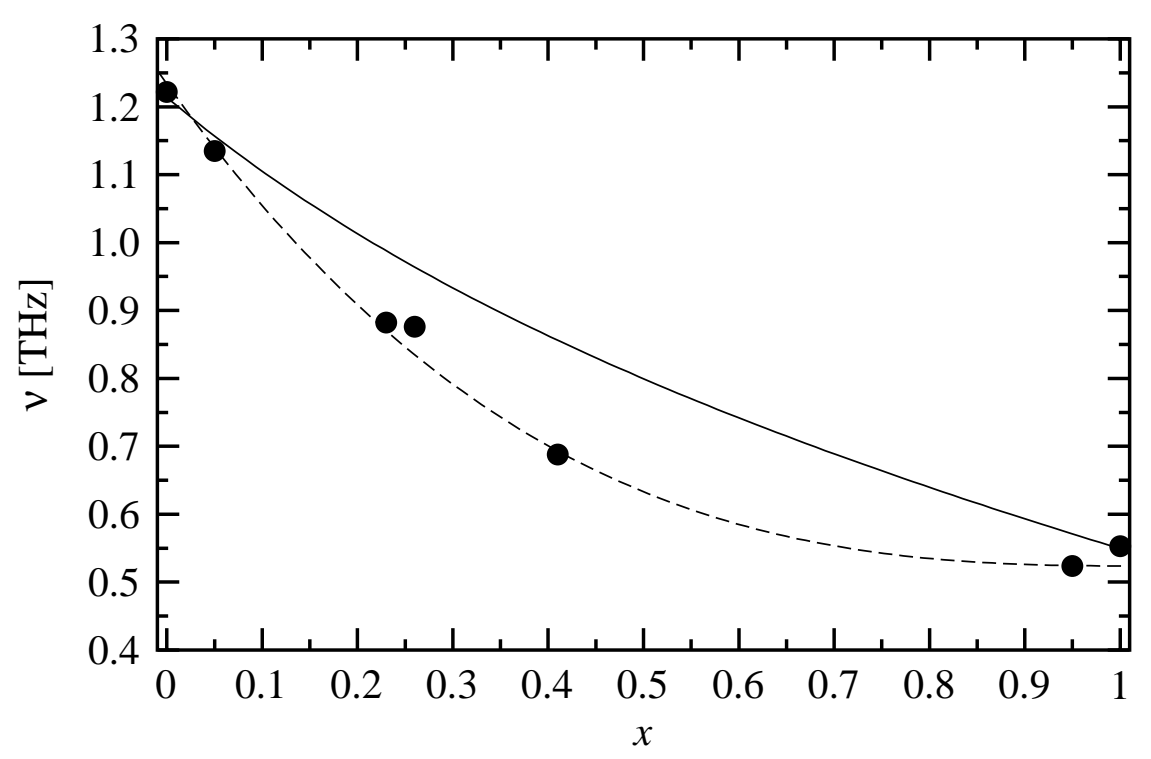

Abbildung 4.4: Vergleich zwischen den Ergebnissen nach Gleichung (4.11), durchgezogene Linie, mit den experimentellen Daten. Die gestrichelte Linie sind Ergebnisse nach Gleichung (4.12).

tistisch mit $\mathrm{Ag}^{+}$bzw. $\mathrm{Na}^{+}$besetzt sind. Die Gitterdynamik der TA Phononen weicht dabei deutlich von der linearen Näherung zwischen den reinen Substanzen ab. Dabei ändert sich die Frequenz für die natriumreichen Zusammensetzungen mit der Konzentration stärker als für die silberreichen Zusammensetzungen. In den Spektren der kinetischen Experimente werden sich Konzentrationsänderungen im Bereich von 50$100 \% \mathrm{AgCl}$-Anteil nur schwächer durch eine Frequenzverschiebung bemerkbar machen. Dafür werden sich für $x<0.5$ kleine Änderungen stark auswirken. Der Nachteil der geringen $v(x)$-Abhängigkeit kann experimentell zum Teil kompensiert werden: In den Phononenspektren ist nach Gleichung (1.37) die Intensität bei kleinen Frequenzen (=Ag-reiche Phasen) höher als bei höheren Frequenzen ( $=N a$-reiche Phasen). So können aufgrund der höheren Intensität dennoch kleine Frequenzänderungen detektiert werden. Analog ist bei höheren Frequenzen die Statistik zwar schlechter, jedoch sind die Frequenzänderungen entsprechend größer.

\subsection{Braggreflexe nach langen Auslagerungszeiten}

Am Ende der Kristallzucht wurden die Kristalle weit oberhalb der Binodalen homogenisiert und anschließend auf Raumtemperatur abgekühlt. Nach den Ergebissen aus den SANS-Experimenten sind die Kristalle dabei entmischt und haben die Konzentrationen der kohärenten Binodalen $x_{1}^{\prime}$ und $x_{2}^{\prime}$ erreicht. Zwischen der Kristallzucht und den 


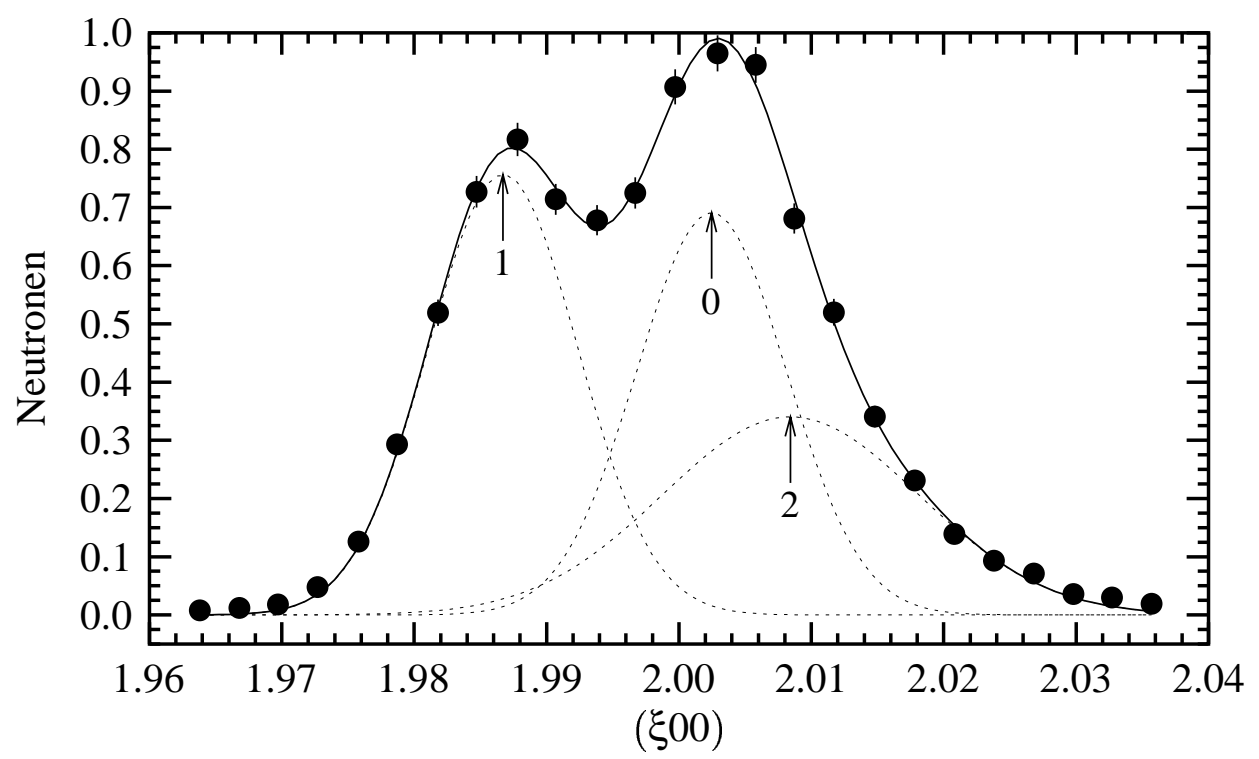

Abbildung 4.5: Der (200)-Braggreflex für $x_{0}=0.41$ nach langen Auslagerungszeiten. Den drei Maxima der angepaßten Gaußkurven entsprechen die Gitterkonstanten $a_{1}=5.598 \AA$, $a_{0}=$ $5.554 \AA$ und $a_{2}=5.538 \AA$.

Neutronenstreuexperimenten lagen dann mehrere Wochen bis Monate. Während dieser Zeit befand sich die Probe im Zweiphasengebiet des Phasendiagramms und konnte, wenn auch nur sehr langsam, weiter entmischen. Die zu erwartenden Zusammensetzungen der natriumreichen und silberreichen Phase liegen infolgedessen im Bereich von $x_{1} \ldots x_{1}^{\prime}$ bzw. $x_{2}^{\prime} \ldots x_{2}$; vgl. Abbildung 2.4.

In den Abbildungen 4.5 und 4.6 ist der (2 00 )-Braggreflex nach langen Auslagerungszeiten für die Proben mit $x_{0}=0.41$ und $x_{0}=0.23$ gezeigt. In den Diffraktogrammen ist die gemessene Intensität gegen den Streuvektor in Einheiten von $2 \pi / a_{\text {nom }}$ aufgetragen. Durch eine unterschiedliche Wahl die von $a_{\text {nom }}$ liegt das Maximum in beiden Diffraktogrammen ungefähr bei $(200)$. Der Braggreflex für die $41 \%$-Probe hat zwei deutliche Maxima. An das Spektrum können drei Gaußkurven ${ }^{4}$ angepaßt werden. Im Vergleich mit den reinen Substanzen sind die natriumreichen Phasen diejenigen mit der größeren Gitterkonstante bzw. mit dem kleineren Wellenvektor. Entsprechend haben die silberreichen Phasen den größeren Wellenvektor. Die Reflexaufspaltung beträgt $\Delta a_{1,2} / a=1.1 \%$. Die Halbwertsbreiten sind deutlich breiter als die Auflösung, die $\Delta_{\| \xi}=0.006$ beträgt: $\Delta \xi_{1}=0.013, \Delta \xi_{0}=0.013$ und $\Delta \xi_{2}=0.028$. Die natriumreiche Phase ist deutlich geringer verbreitert als die silberreiche Phase.

Das Diffraktogramm bei $x_{0}=0.23$ weicht vom Diffraktogramm bei $x_{0}=0.41 \mathrm{ab}$. Neben einem zentralen Maximum ist eine breite Schulter zu kleineren Gitterkonstanten

${ }^{4}$ Die Gaußfunktionen entspricht dem Typ $I(x)=I_{\max } \cdot \mathrm{e}^{-4 \ln (2)\left(\frac{x-x_{\max }}{\Delta x}\right)^{2}}$. 


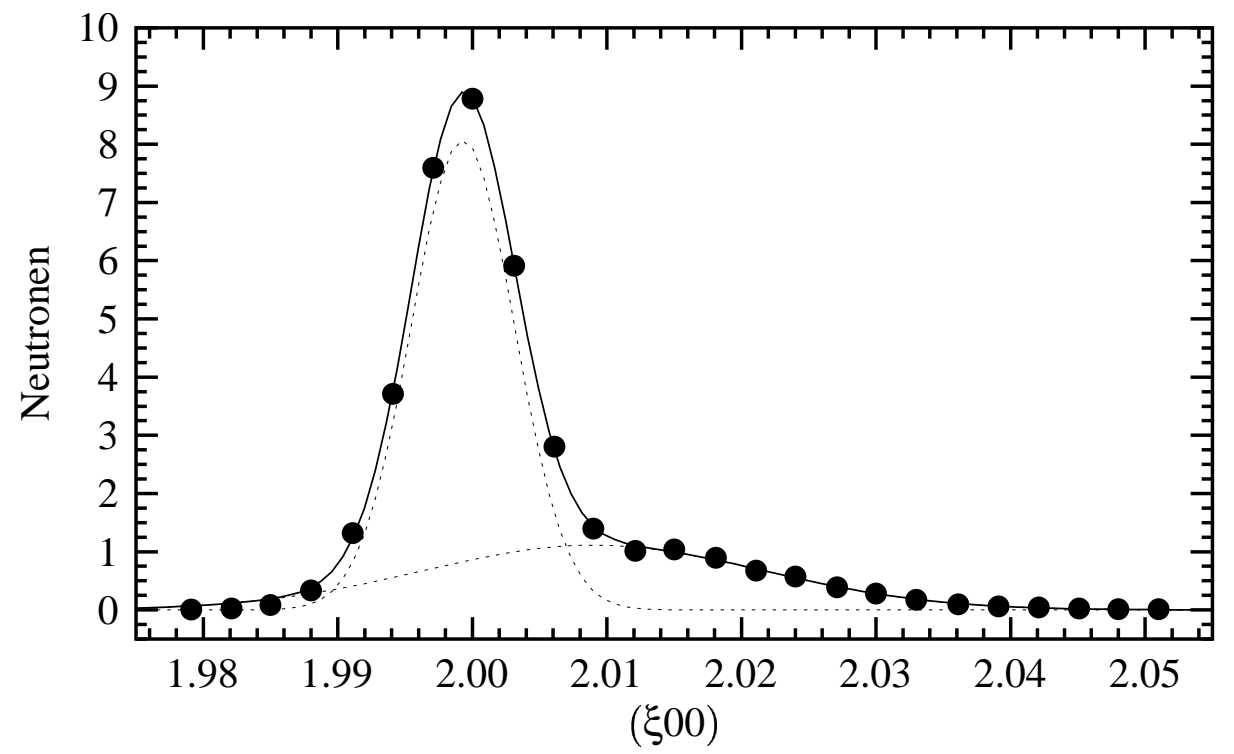

Abbildung 4.6: Der (2 0 0)-Braggreflex für $x_{0}=0.23$ nach langen Auslagerungszeiten. Die den maximalen Intensitäten entsprechenden Wellenvektorüberträge sind die Gitterkonstanten, die $a_{1}=5.608 \AA$ und $a_{2}=5.581 \AA$ zuzuordnen.

zu erkennen, und der Reflex läßt sich mit nur zwei Gaußkurven unterschiedlicher Halbwertsbreite beschreiben. Die Reflexaufspaltung beträgt $\Delta a / a=0.5 \%$. Die Halbwertsbreiten $\Delta \xi_{1}=0.009$ und $\Delta \xi_{2}=0.030$ sind deutlich größer als die Auflösungsbreite $\Delta_{\| \xi}=0.006$. Es ist wieder die silberreiche Phase gegenüber der natriumreichen Phase verbreitert.

Als Ursache für die Verbreiterung der Reflexe in beiden Proben kommen Gitterkonstantenverteilungen oder Korngrößeneffekte in Betracht. Bei einer Verbreiterung der Braggreflexe durch Korngrößeneffekte kann der Durchmesser $D$ der Körner mit Hilfe der Scherrer-Gleichung [Cullity 1978] berechnet werden. Hierzu muß zunächst die Halbwertsbreite des Reflexes bezüglich der Geräteauflösung entfaltet werden. Bei einer gerätebedingten Auflösung von $\Delta_{\| \xi}$ ist die entfaltete Breite des Reflexes

$$
\Delta \Xi=\sqrt{(\Delta \xi)^{2}-\left(\Delta_{\| \xi}\right)^{2}}
$$

wenn die Streu- und die Auflösungsfunktion gaußisch sind. Der Korndurchmesser berechnet sich dann in Abhängigkeit vom Streuwinkel $\theta$, der Halbwertsbreite des Reflexes $\Delta_{2 \theta}$ im Bogenmaß und der Wellenlänge $\lambda$ der Neutronen zu

$$
D=\frac{0.89 \cdot \lambda}{\Delta_{2 \theta} \cos (\theta)} .
$$


Wenn die Verbreiterung der Braggreflexe durch Korngrößeneffekte verursacht sind, haben die Körner in der $41 \%$-Probe einen Durchmesser von $D_{1}=833 \AA, D_{0}=833 \AA$ und $D_{2}=450 \AA$ bzw. in Probe mit $x_{0}=0.23$ sind die Durchmesser $D_{1}=1480 \AA$ und $D_{2}=340 \AA$ A. Bei der Behandlung der kinetischen Experimente im Abschnitt 5.1, S. 76, wird sich zeigen, daß die Körner nach langen Auslagerungszeiten deutlich größer als die hier berechneten Werte sind. Die Verbreiterung durch Korngrößeneffekte ist folglich auszuschließen.

Werden für Raumtemperatur die Gitterkonstantenaufspaltungen für die kohärente Spinodale $x_{1}^{\prime}=0.2$ und $x_{1}^{\prime}=0.8$ und die Binodale $x_{1}=0.05$ und $x_{2}=0.95$ berechnet, grenzen sie den Bereich der Aufspaltung auf 1-1.5\% ein. Das Kristallgitter der $41 \%$-Probe hat nach den langen Auslagerungszeiten begonnen zu relaxieren. Neben der Gitterkonstanten der homogenen Phase finden sich ferner die Gitterkonstante der beiden entmischten Phasen. Deren Maxima entsprechen immer noch Gitterkonstanten von Konzentrationen, die in der Nähe der kohärenten Spinodalen liegen. Es ist demnach darauf zu schließen, daß sich der schnellen chemischen Entmischung bis $x_{1}^{\prime}$ und $x_{2}^{\prime}$ die Relaxation des Kristallgitters anschließt. Die Kinetik der Gitterrelaxation vollzieht sich auf einer Zeitskala die sich um mehr als eine Größenordnung von der Skala der chemischen Entmischung unterscheidet.

Im Fall der $23 \%$-Probe ist die Aufspaltung um den Faktor zwei kleiner. Wenn die silber- und natriumreiche Phase die Konzentrationen $x_{1}^{\prime}$ und $x_{2}^{\prime}$ mit der dazugehörigen Gitterkonstanten haben, ist das Intensitätsverhältnis in Abhängigkeit von der mittleren Streulänge $b(x)=x b_{A g}+(1-x) b_{N a}+b_{C l}$ und dem Streuvolumenanteil $V(x)$ bei gleicher Auflösung für beide Reflexe

$$
\frac{I\left(x_{1}^{\prime}\right)}{I\left(x_{2}^{\prime}\right)} \propto \frac{V\left(x_{1}^{\prime}\right)}{V\left(x_{2}^{\prime}\right)} \frac{\left|b\left(x_{1}^{\prime}\right)\right|^{2}}{\left|b\left(x_{2}^{\prime}\right)\right|^{2}}=\frac{32}{1} .
$$

Die für die silberreiche Phase zu erwartende Intensität ist im Verhältnis sehr gering. Im Vergleich mit dem anderen Kristall ergibt sich, daß auch hier drei dominierende Gitterkonstanten vorliegen. Dem Maximum ist die natriumreiche Phase mit Gitterkonstanten $a\left(x_{1} \ldots x_{0}\right)$ und den Zusammensetungen $x_{1} \ldots x_{1}^{\prime}$ zuzuordnen. Das Nebenmaximum entspricht der silberreichen Phase mit einer Gitterkonstantenverteilung von $a\left(x_{0} \ldots x_{2}\right)$ und Konzentrationen im Intervall $x_{2}^{\prime} \ldots x_{2}$. Über diese Experimente hinaus wurde ein Fragmentstïck des Kristalls mit $x_{0}=0.26$ nach langen Auslagerungszeiten auf Diffuse Streuung hin untersucht. Es ergaben sich aber keine neuen Erkenntnisse ${ }^{5}$.

In beiden Kristallen liegen Gitterkonstantenverteilungen vor, die um die Gitterkonstanten zentriert sind, die Zusammensetzungen von $x_{0}, x_{1}^{\prime}$ und $x_{2}^{\prime}$ entsprechen. Dies ist konsistent zu den früheren Experimenten an den 50 \%-Pulverproben [Caspary 1998, Caspary u. a. 2001], bei denen nach Stunden die einsetzende Gitterrelaxation anhand der langsam zunehmenden Reflexbreite beobachtet wurde.

\footnotetext{
${ }^{5}$ Im Anhang in Abbildung .5 ist das Diffraktogramm für die $\left[\begin{array}{lll}0 & 1 & 1\end{array}\right]$-Ebene gezeigt.
} 


\subsection{Dispersionskurven nach langen Auslagerungszeiten}

Abbildung 4.7 zeigt zwei Spektren mit $x_{0}=0.23$ und $x_{0}=0.41$ nach langen Auslagerungszeiten bei Raumtemperatur. In beiden Spektren sind jeweils zwei Phononen bei jeweils $\pm v$ und die inkohärent elastische Linie bei $v=0$ sehr gut zu erkennen. Die Spektren sind für positive und negative Energieüberträge aufgenommen, die durch das Vorzeichen von $v$ gekennzeichnet sind. Die Frequenzen bei $\pm v$ gehören folglich zum gleichen Phonon. Zum Vergleich sind wieder die Frequenzlagen der reinen Substanzen mit Pfeilen markiert. Die linke Seite der Spektren mit $\Delta E<0$ ist die sogenannte fokussierende Seite des Spektrometers. Für die Messungen mit dem Energieübertrag vom Neutron auf die Probe ist die gerätebedingte Auflösung des Spektrums besser als für $\Delta E>0$. Aus diesem Grund wurden die später behandelten kinetischen Experimente mit für $\Delta E<0$ durchgeführt.

Im Vergleich der Frequenzen mit maximaler Intensität mit den Frequenzen der reinen Substanzen ist die $\mathrm{Na}$-reiche Phase dem Phonon mit höherer Frequenz und die Agreiche Phase dem Phonon mit der niedrigeren Frequenz zuzuordnen. Aufgrund der gut definierten Phononen ist auf zwei Phasen zu schließen, in denen eine schmale Konzentrationsverteilung vorliegt. Für eine noch immer merklich vorhandene homogene Phase gibt es keine Hinweise: Es müßte dann eine Intensität zwischen den Phononen der entmischten Phasen zu finden sein.

Eine Anpassung von vier Lorenzfunktionen für die Phononen und einer Gaußfunktion für die inkohärent elastische Linie an das Spektrum ergab:

\begin{tabular}{rrrrrrr}
\hline \hline & \multicolumn{3}{c}{ natriumreiche Phase } & \multicolumn{4}{c}{ silberreiche Phase } \\
$x_{0}$ & $v_{\max }[\mathrm{THz}]$ & $\Delta v[\mathrm{THz}]$ & $I_{\max }$ & $\mathrm{I} v_{\max }[\mathrm{THz}]$ & $\Delta v[\mathrm{THz}]$ & $I_{\max }$ \\
\hline \hline \multirow{2}{*}{0.23} & $-0.841 \pm 0.005$ & $0.12 \pm 0.01$ & 178 & $-0.405 \pm 0.004$ & $0.11 \pm 0.01$ & 182 \\
& $0.884 \pm 0.005$ & $0.21 \pm 0.02$ & 184 & $0.420 \pm 0.005$ & $0.15 \pm 0.01$ & 185 \\
& & & & & & \\
0.41 & $-0.842 \pm 0.018$ & $0.20 \pm 0.07$ & 30 & $-0.390 \pm 0.002$ & $0.15 \pm 0.01$ & 133 \\
& $0.854 \pm 0.009$ & $0.24 \pm 0.03$ & 51 & $0.431 \pm 0.002$ & $0.12 \pm 0.01$ & 180 \\
\hline
\end{tabular}

Zunächst fallen die nahezu gleichen maximalen Intensitäten für das Spektrum der $x_{0}=0.23$ Probe auf. Für die Binodalzusammensetzungen $x_{1}=0.05$ und $x_{2}^{\prime}=0.95$ kann das Intensitätsverhältnis mit

$$
\frac{\Delta \mathrm{v}_{1} \cdot I_{\max , 1}}{\Delta \mathrm{v}_{2} \cdot I_{\max , 2}} \approx \frac{V\left(x_{1}^{\prime}\right)}{V\left(x_{2}^{\prime}\right)} \frac{\left|b\left(x_{1}^{\prime}\right)\right|^{2}}{\left|b\left(x_{2}^{\prime}\right)\right|^{2}} \frac{v_{\max , 2}^{2}}{v_{\max , 1}^{2}}=1.4
$$

abgeschätzt werden. Die annähernd gleichen maximalen Intensitäten sind demgemäß auf das unterschiedliche Streuvermögen der entmischten Phasen, deren Volumenanteil 

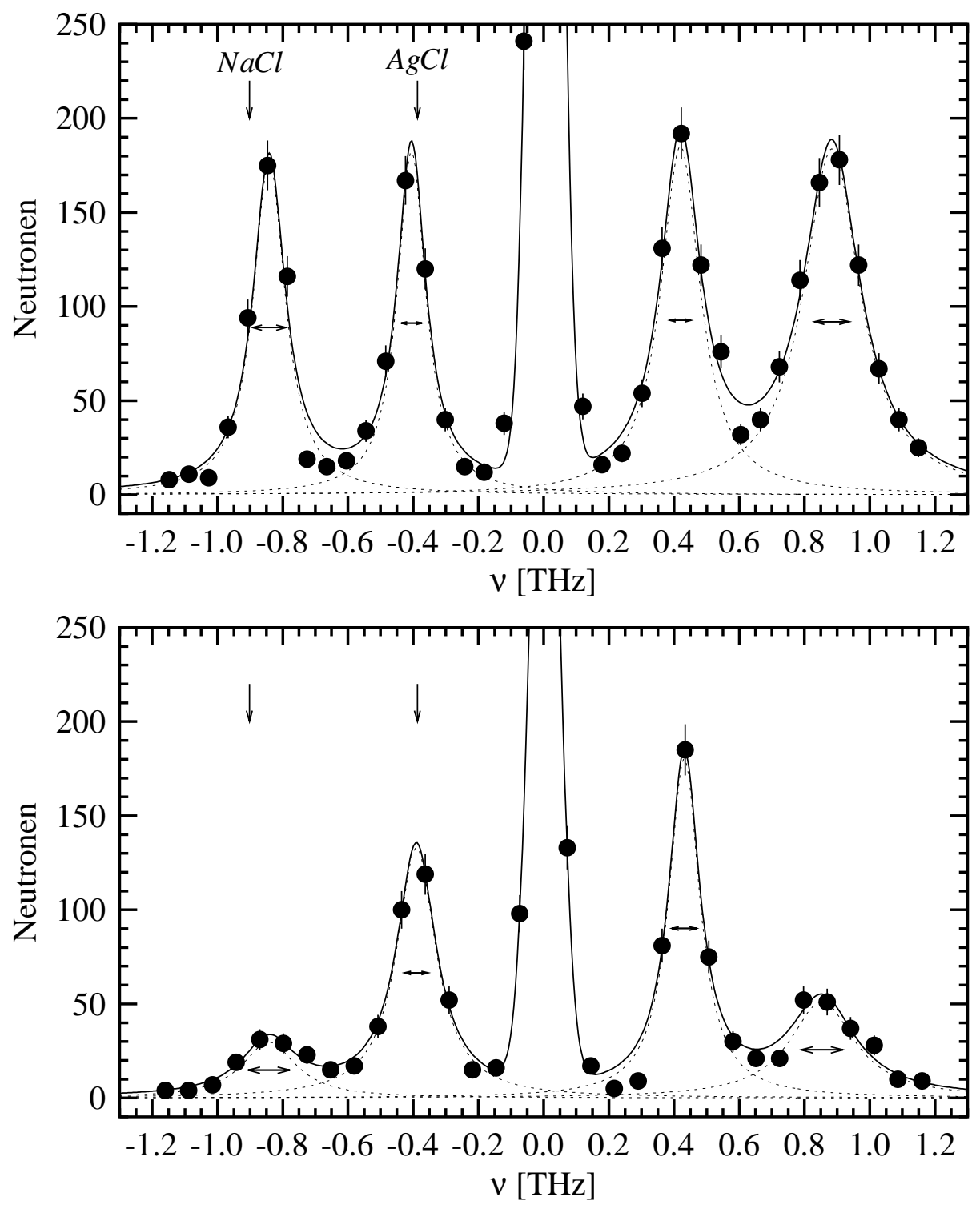

Abbildung 4.7: Die Spektren für die TA Phononen für $x_{0}=0.23$ (oben) und $x_{0}=0.41$ (unten) bei $\xi=(20.20)$ und Raumtemperatur nach langen Auslagerungszeiten. Es sind deutlich die zur silber- und natriumreichen Phase gehörenden Phononen und die inkohärent elastische Linie zu erkennen. Die nahezu gleichen Intensitäten der Phononen für die natriumreiche und die silberreiche Phase für $x_{0}=0.23$ ist auf die Frequenzabhängigkeit der Intensität, das Volumenverhältnis der beiden Phasen und die unterschiedlichen Streulängen zurückzuführen. Zur besseren Einordnung sind die Frequenzlagen der reinen Komponenten mit Pfeilen markiert. Die Gräteauflösung $\Delta_{v}$ ist jeweils als Doppelpfeil mit aufgetragen. 
und die Frequenzlage zurückzuführen. Für Zusammensetzungen der kohärenten Spinodale $x_{1}^{\prime}=0.2$ und $x_{2}^{\prime}=0.8$ wäre das Intensitätsverhältnis mit 0.7 anzugeben. Die experimentellen Ergebnisse sind für $x_{0}=0.23 \tilde{I}_{1} / \tilde{I}_{2}=1.1$ bzw. $\tilde{I}_{1} / \tilde{I}_{2}=1.4$. Den experimentellen Werten für die $41 \%$-Probe mit Verhältnissen von 0.3 und 0.6 steht der berechnete Werte von 0.5 für die Binodalkonzentrationen bzw. 0.7 für die kohärente Binodale gegenüber. Eine Abschätzung der mittleren Konzentrationen anhand der Integralen Intensitäten führt zu Zusammensetzungen, die dichter an den Binodalzusammensetzungen als an der kohärenten Spinodalkonzentration liegen. Das bedeutet, daß in beiden Proben die chemische Entmischung weiter fortgeschritten ist. Die Konzentrationen sind nicht mehr die der kohärenten Binodalen, sondern nähern sich bereits der Binodalkonzentration an. Insbesondere gilt das für natriumreiche Phase, die empfindlichen mit Frequenzänderungen auf Änderung der Konzentration reagiert; vgl. Abbildung 4.4.

Die Halbwertsbreiten der Phononen nach langen Auslagerungszeiten sind jeweils größer als die Auflösung $\Delta_{v}$ des Spektrometers, die in Abbildung 4.7 als Doppelpfeile für jedes Signal mit aufgetragen ist. Es fällt auf, daß in drei Fällen die relative Verbreiterung der Halbwertsbreite $\Delta v / \Delta_{v}$ des zur $A g$-reichen Phase gehörenden Phonons größer ist als die Verbreiterung des zur $N a$-reichen Phase gehörenden Phonons. Die Ausnahme bei $x_{0}=0.41$ und positivem Energieübertrag ist aber auf den Meßwert bei $0.8 \mathrm{THz}$ zurückzuführen. Es liegt im Vergleich mit den umliegenden Meßwerten relativ hoch und führt so zu einer Vergrößerung der Halbwertsbreite. Als Ursachen für die Verbreiterung der Halbwertsbreiten kommen die Gitterkonstantenverteilungen, Konzentrationsgradienten innerhalb der entmischten Phasen und eine Dämpfung der Phononen in Betracht.

Bei einer Änderung der Gitterkonstanten um $0.5 \%$ ändert sich das Volumen um $1.5 \%$. Eine derartige Volumenänderung führt mit einer Grüneisenkonstante ${ }^{6} \gamma \approx 2 \mathrm{zu}$ einer Frequenzänderung von

$$
\gamma=\frac{\mathrm{d} \ln v}{\mathrm{~d} \ln V}=\frac{\frac{1}{v} \mathrm{~d} v}{\frac{1}{V} \mathrm{~d} V} \quad \Rightarrow \quad \frac{\Delta v}{v}=\gamma \frac{\Delta V}{V}=3 \% .
$$

Eine Verbreiterung der Phononenhalbwertsbreiten aufgrund von Verteilungen der Gitterkonstanten wären mit den Experimenten nicht nachweisbar und können vernachlässigt werden. Nach den Intensitätsverhältnissen können in den Phasen nach langen Auslagerungszeiten noch kleine Konzentrationsgradienten im Bereich $x_{1} \ldots x_{1}^{\prime}$ und $x_{2}^{\prime} \ldots x_{2}$ vorliegen. Sie können aber nicht den entscheidenden Beitrag zu Verbreiterung in beiden Phasen liefern, da dann nach Abbildung 4.4 die natriumreiche und nicht die silberreiche Phase verbreitert sein muß.

Nimmt man die Debye-Temperatur oder den Schmelzpunkt als ein Maß für die An-

\footnotetext{
${ }^{6}$ Die Grüneisenkonstanten der reinen Substanzen bei $10^{\circ} \mathrm{C}$ sind $\gamma_{\mathrm{AgCl}}=1.9$ und $\gamma_{\mathrm{NaCl}}=1.6[\mathrm{Gmelin}$ 1971].
} 
harmonizität einer Substanz, ist Silberchlorid die anharmonischere Komponente. So sind z.B. die Debye-Temperaturen $\Theta_{\mathrm{D}}^{\mathrm{AgCl}}=-93{ }^{\circ} \mathrm{C}$ und $\Theta_{\mathrm{D}}^{\mathrm{NaCl}}=20^{\circ} \mathrm{C}$. Die Anharmonizität spiegelt sich in den Spektren als Dämpfung wider, die zu einer Verbreiterung der Phononenspektren führt. Die größere relative Verbreiterung der silberreichen Phase ist also auf anharmonische Effekte zurückzuführen.

Die Zusammensetzungen der Phasen nach sollten langen Auslagerungszeiten unabhängig von der mittleren Zusammensetzung der Kristalle sein, und folglich müssen die Frequenzenlagen unabhängig von $x_{0}$ sein. Um dies zu verifizieren, wurden die Dispersion der TA Phononen in $\left[\begin{array}{lll}1 & 0 & 0\end{array}\right],\left[\begin{array}{lll}1 & 1 & 0\end{array}\right]$ für die drei Proben gemessen. Es konnte so experimentell bestätigt werden, wie in Abbildung $4.8 \mathrm{zu}$ sehen ist, daß die Frequenzen nach langen Auslagerungszeiten unabhängig von der mittleren Konzentration sind. Um den Verlauf der Dispersion in den zwei Phasen zu beschreiben, wurde die Linearkombination

$$
\mathrm{v}_{i}(\xi)=x_{i} \cdot \mathrm{v}_{A g C l}(\xi)+\left(1-x_{i}\right) \cdot v_{N a C l}(\xi)
$$

mit den Frequenzen der reinen Substanzen $v_{A g C l}(\xi), v_{N a C l}(\xi)$ und deren Stoffmengenenanteilen $x_{i}$ angesetzt. Dies ist hier zulässig, weil die gemessenen Phononenfrequenzen sehr dicht an den Frequenzen der reinen Substanzen liegen. Schon die Annahme der Binodalzusammensetzungen von $x_{1}=0.05$ und $x_{2}=0.95$ führt zu einem guten Ergebnis. Werden die Stoffmengenanteile an die entsprechende Dispersion angepaßt, werden für die Zusammensetzungen $x_{1}=0.11 \pm 0.02$ und $x_{2}=0.98 \pm 0.1$ erhalten. Die Abweichungen für $\xi \rightarrow 0$ zwischen der angepaßten Dispersion und der Dispersionskurve, die aus den elastischen Konstante extrapoliert ist, ist auf Ableseungenauigkeiten der Dispersionskurven aus Auftragungen in der Literatur zurückzuführen. Zudem können Unterschiede zwischen den experimentell bestimmten elastischen Konstanten auftreten, je nachdem ob sie mit Ultraschalluntersuchungen oder mit Neutronenstreuung bestimmt wurden [Cowley 1967].

Zudem können aus diesen Daten wieder die Schallgeschwindigkeiten und die elastischen Konstanten analog zur homogenen Phase berechnet werden. Da in den Kristallen

Tabelle 4.2: Für Raumtemperatur prognostizierte Schallgeschwindigkeiten und elastischen Konstante

\begin{tabular}{lccc}
\hline \hline$x$ & & $0.05 \ldots 0.20$ & $0.75 \ldots 0.95$ \\
\hline \hline$c_{44}$ & {$[\mathrm{GPa}]$} & $11.5 \ldots 12.5$ & $6.55 \ldots 7.87$ \\
$v^{\mathrm{TA}[100] /[110]}$ & {$[\mathrm{m} / \mathrm{s}]$} & $2157 \ldots 2363$ & $1124 \ldots 1399$ \\
$c_{11}-c_{12}+c_{44}$ & {$[\mathrm{GPa}]$} & $45.2 \ldots 48.1$ & $30.7 \ldots 34.6$ \\
$v^{\mathrm{TA}[111]}$ & {$[\mathrm{m} / \mathrm{s}]$} & $2608 \ldots 2466$ & $1615 \ldots 1473$ \\
\hline
\end{tabular}




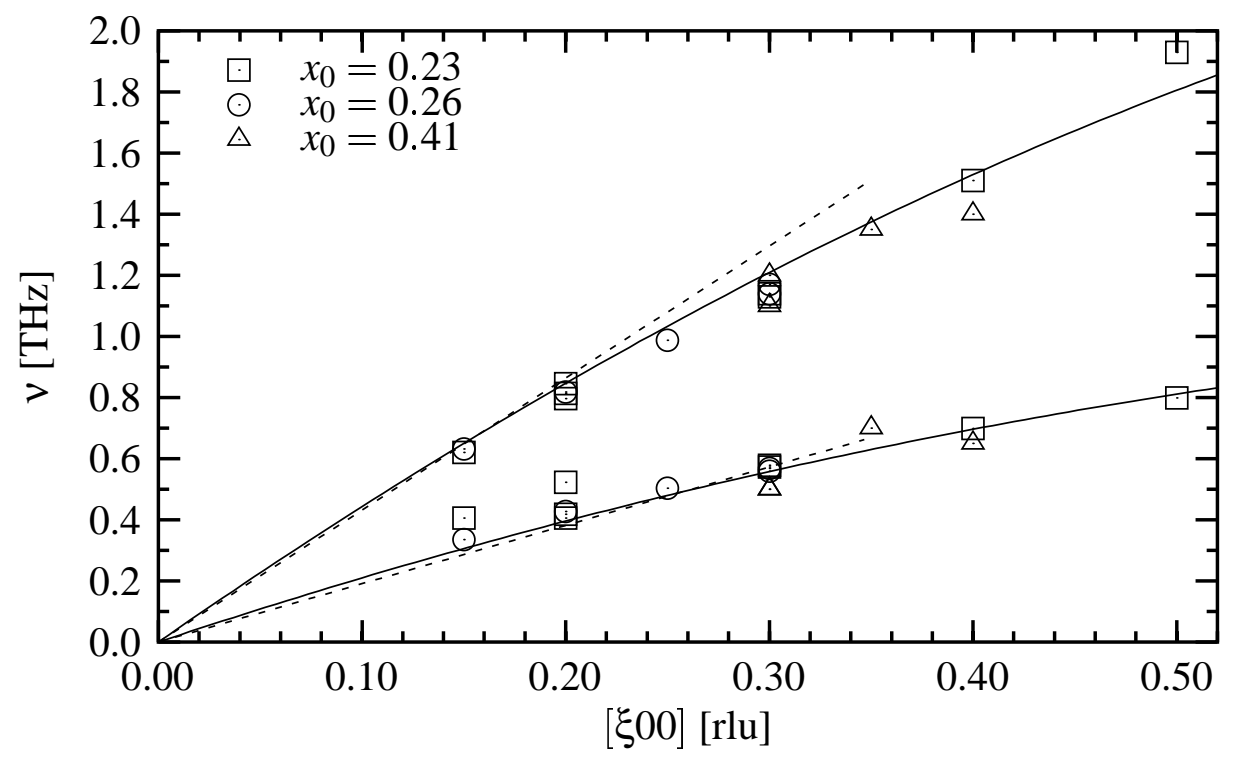

Abbildung 4.8: Die Dispersionskurven nach langen Auslagerungszeiten für $x_{0}=0.23, x_{0}=0.26$ und $x_{0}=0.41$ in $\left[\begin{array}{lll}1 & 0 & 0\end{array}\right]$ bei Raumtemperatur. Die durchgezogenen Linien sind Liniarkombinationen der Dispersion der reinen Substanzen mit $x_{1}=0.05$ und $x_{2}=0.95$ als Wichtungsfaktoren, die die gemessenen Dispersionen gut beschreiben. Die gestrichelte Linie entspricht der aus den elastischen Konstanten linear extrapolieren Dispersion. Im Angang sind auf Seite 107 zusätzlich die Dispersionkurve bis zum Zonenrand für $x_{0}=0.23$ und die Dispersion in $\left[\begin{array}{lll}1 & 1 & 0\end{array}\right]$ für $x_{0}=0.26$ gezeigt.

Gitterkonstanten- und Konzentrationsverteilungen vorliegen, sind die Massendichten innerhalb der beiden Phasen nicht konstant. Um dem Rechnung zu tragen, wird der Wertebereich für die Stoffmengenanteile zur Berechnung der Massendichte nach Gleichung (4.2) auf den Bereich zwischen der kohärenten Binodale und der Binodale erweitert: $x_{1}=0.05 \ldots 0.20$ für die natriumreiche und $x_{2}=0.75 \ldots 0.95$ silberreiche Phase. So werden die möglichen Dichten, die zwischen den frühen Stadien und dem entmischten Zustand auftreten können, erfaßt. Für die Schallgeschwindigkeiten und elastischen Konstanten können dann die in Tabelle 4.2 zusammengefaßten Werte prognostiziert werden.

Beim Vergleich der experimentellen Ergebnisse in Tabelle 4.3 untereinander fallen keine Inkonsistenzen auf. Für die Proben mit unterschiedlichen mittleren Zusammensetzungen ergeben sich in guter Übereinstimmung die gleichen Schallgeschwindigkeiten und elastischen Konstanten. Auch der relative Unterschied der beiden entmischten Phasen zueinander mit einem Faktor zwei für die Schallgeschwindigkeiten und elastischen Konstanten stimmt mit den Verhältnissen bei den reinen Substanzen überein. Beim Vergleich der Absolutwerte mit der Vorhersage sind jedoch für die natriumreiche Phase Abweichungen von den vorhergesagten Werten zu finden. Die experimentellen 
Ergebnisse sind deutlich erhöht, und die Ergebnisse für die silberreiche Phase liegen im oberen Teil des vorhergesagten Wertebereichs. Als Erklärung kommen, wie für die Dispersionskurven im homogenen Zustand in Abschnitt 4.1, die schlechte Bestimmung des Dispersionsverlaufs für sehr kleine Wellenvektoren in Betracht. Es werden wieder elastische Konstanten aus Neutronenstreuexperimenten mit Konstanten verglichen, die aus Ultraschalluntersuchungen stammen. Hinzu kommt weiterhin die Massendichteverteilung.

Für den Zustand nach langen Auslagerungszeiten ergibt sich zusammenfassend folgendes Bild: In der Probe liegen eine natriumreiche und ein silberreiche Phase vor, deren Zusammensetzungen gegen die Binodalkonzentrationen gehen. Jeweils ein Teil der beiden Phasen bildet ein Kristallgitter mit einer Gitterkonstanten, die der Gitterkonstanten der mittleren Konzentration $x_{0}$ gleichkommt. Der andere Teil der jeweiligen Phase hat Gitterkonstanten, die Konzentrationsverteilungen von $x_{1} \ldots x_{0}$ bzw. $x_{0} \ldots x_{2}$ entsprechen. Im Anschluß an die schnelle chemische Entmischung bei konstanter Gitterkonstante wird die Entmischung langsam fortgesetzt. Dabei beginnt das Kristallgitter zu relaxieren. Es werden in den untersuchten Zeiträumen nicht die Gitterkonstanten erreicht, die den Konzentrationen entsprechen. Im Vergleich mit der Zeitskala der schnellen Entmischung, wie sie die SANS-Ergebnisse festlegen, kann bei diesem Zustand nach langen Auslagerungszeiten von einem kinetisch stabilisierten, quasistabilen Zustand gesprochen werden. 
Tabelle 4.3: Die Schallgeschwindigkeiten und elastischen Konstanten der entmischten Phasen nach langen Auslagerungszeiten berechnet für $T=20{ }^{\circ} \mathrm{C}$ mit $x_{1}=0.05 \ldots 0.20$ und $x_{2}=0.75 \ldots 0.95$. Die Gitterkonstanten sind die experimentell bestimmte Werte.

\begin{tabular}{|c|c|c|c|c|}
\hline \multicolumn{2}{|c|}{$x_{0}=0.23$} & \multicolumn{2}{|l|}{$\begin{array}{r}\mathrm{TA}\left[\begin{array}{lll}1 & 0 & 0\end{array}\right] \\
c=c_{44}\end{array}$} & \\
\hline$a$ & {$[\AA]$} & 5.612 & & \\
\hline$\rho_{1}$ & {$\left[\mathrm{~kg} / \mathrm{m}^{3}\right]$} & $2355 \ldots 2834$ & & \\
\hline$\rho_{2}$ & {$\left[\mathrm{~kg} / \mathrm{m}^{3}\right]$} & $4588 \ldots 5227$ & & \\
\hline$(\partial v / \partial \xi)_{1}$ & {$[\mathrm{THz} / \mathrm{rlu}]$} & 4.459 & & \\
\hline$(\partial v / \partial \xi)_{2}$ & {$[\mathrm{THz} / \mathrm{rlu}]$} & 2.285 & & \\
\hline$v_{1}$ & {$[\mathrm{~m} / \mathrm{s}]$} & 2502 & & \\
\hline$v_{2}$ & {$[\mathrm{~m} / \mathrm{s}]$} & 1282 & & \\
\hline$c_{1}$ & [GPa] & $14.7 \ldots 17.7$ & & \\
\hline$c_{2}$ & {$[\mathrm{GPa}]$} & $7.54 \ldots 8.59$ & & \\
\hline $\mathbf{x}_{\mathbf{0}}=$ & .26 & $\mathrm{TA}\left[\begin{array}{lll}1 & 0 & 0\end{array}\right]$ & $\mathrm{TA}\left[\begin{array}{lll}1 & 1 & 0\end{array}\right]$ & $\mathrm{TA}\left[\begin{array}{lll}1 & 1 & 1\end{array}\right]$ \\
\hline & & & $c=c_{44}$ & $c=c_{11}-c_{12}+c_{44}$ \\
\hline$a$ & {$[\AA]$} & 5.610 & 5.610 & 5.613 \\
\hline$\rho_{1}$ & {$\left[\mathrm{~kg} / \mathrm{m}^{3}\right]$} & $2358 \ldots 2837$ & $2358 \ldots 2837$ & \\
\hline$\rho_{2}$ & {$\left[\mathrm{~kg} / \mathrm{m}^{3}\right]$} & $4593 \ldots 5232$ & $4593 \ldots 5232$ & $4593 \ldots 5224$ \\
\hline$(\partial v / \partial \xi)_{1}$ & {$[\mathrm{THz} / \mathrm{rlu}]$} & 4.459 & 5.767 & \\
\hline$(\partial v / \partial \xi)_{2}$ & {$[\mathrm{THz} / \mathrm{rlu}]$} & 2.285 & 3.235 & $5.347^{a}$ \\
\hline$v_{1}$ & {$[\mathrm{~m} / \mathrm{s}]$} & 2501 & 2288 & \\
\hline$v_{2}$ & {$[\mathrm{~m} / \mathrm{s}]$} & 1282 & 1283 & 1733 \\
\hline$c_{1}$ & {$[\mathrm{GPa}]$} & $14.7 \ldots 17.7$ & $12.3 \ldots 14.9$ & \\
\hline$c_{2}$ & {$[\mathrm{GPa}]$} & $7.55 \ldots 8.60$ & $7.56 \ldots 8.61$ & $41.4 \ldots 47.1$ \\
\hline $\mathbf{x}_{\mathbf{0}}=$ & .41 & $\mathrm{TA}\left[\begin{array}{lll}1 & 0 & 0\end{array}\right]$ & & \\
\hline$a$ & {$[\AA]$} & 5.594 & & \\
\hline$\rho_{1}$ & {$\left[\mathrm{~kg} / \mathrm{m}^{3}\right]$} & $2379 \ldots 2862$ & & \\
\hline$\rho_{2}$ & {$\left[\mathrm{~kg} / \mathrm{m}^{3}\right]$} & $4633 \ldots 5277$ & & \\
\hline$(\partial v / \partial \xi)_{1}$ & {$[\mathrm{THz} / \mathrm{rlu}]$} & 4.459 & & \\
\hline$(\partial v / \partial \xi)_{2}$ & {$[\mathrm{THz} / \mathrm{rlu}]$} & 2.285 & & \\
\hline$v_{1}$ & {$[\mathrm{~m} / \mathrm{s}]$} & 2494 & & \\
\hline$v_{2}$ & {$[\mathrm{~m} / \mathrm{s}]$} & 1278 & & \\
\hline$c_{1}$ & [GPa] & $14.8 \ldots 17.8$ & & \\
\hline$c_{2}$ & [GPa] & $7.57 \ldots 8.62$ & & \\
\hline
\end{tabular}

${ }^{a}$ mit zwei Meßpunkten bei $(0.08 \mid 0.426)$ und $(0.15 \mid 0.803)$ 


\section{Ergebnisse der kinetischen Experimente}

Die im vorangegangenen Kapitel beschriebenen Ergebnisse der statischen Experimente flankieren die kinetischen Experimente zur Bestimmung der frühen Stadien des Entmischungsmechanismus in Silber-Natriumchlorid. Aus der wohldefinierten homogenen Phase vor der Entmischung gehen die Proben nach den frühen Stadien der Entmischung in den quasistabilen Zustand über. In diesem Kapitel werden nun die Ergebnisse der kinetischen Experimente dargestellt, mit denen die frühen Entmischungsstadien untersucht wurden. In den kinetischen Experimenten wurden die Proben aus dem homogenen Bereich des Phasendiagramms in die Mischungslücke abgeschreckt und die frühen Stadien der Entmischung mittels zeitaufgelöster inelastischer Neutronenstreuung verfolgt.

\subsection{Kinetik im instabilen Bereich}

Der am einfachsten festzulegende Zeitpunkt $t=0$ in den kinetischen Experimenten ist derjenige, in dem die Temperaturänderung beginnt. Wenn jedoch Experimente mit unterschiedlichen Kühlraten z.B. aufgrund unterschiedlicher Probengrößen und Zusammensetzungen miteinander verglichen werden sollen, muß ein Referenzzeitpunkt $\tau$ definiert werden. Eine Möglichkeit wäre, den Nullpunkt mit dem Erreichen der Auslagerungstemperatur zu definieren. Dies ist jedoch schwierig, weil in den Experimenten sich die Temperatur dem Sollwert annähert und bzw. oder um ihn schwingt. Darüber hinaus ist von einer Entmischung auszugehen, wenn die Binodaltemperatur unterschritten wird. Da in den Experimenten beim Abkühlen bis zu Binodaltemperatur nur die temperaturabhängige Frequenzverschiebung beobachtet wurde, wird der Zeitnullpunkt so definiert, daß zu diesem Zeitpunkt die Binodaltemperatur erreicht wird ${ }^{1}: \tau=0$ wenn $T=T_{\text {Binodal }}$. In Tabelle 5.1 sind die Abkühlzeiten für die kinetischen Experimente aufgeführt. Den Zeitkanälen der Breite $\Delta t$ wird als Zeitpunkt die Mitte des jeweiligen Zeitkanals zugeordnet. Während des Abkühlens wurden keine Temperaturgradienten zwischen den Thermoelementen an der Probenoberfläche gemessen, so daß nur Temperaturgradienten von der Probenmitte zur Oberfläche auftraten.

Um einen Anknüpfungspunkt zu den vorhergehenden Arbeiten zu haben, ist kontrolliert worden, ob sich die in den Pulvermessungen [Caspary u. a. 2001] angedeuteten Satellitenreflexe mit Messungen an den Einkristallen bestätigen lassen. Wie erwartet,

\footnotetext{
${ }^{1}$ Die Temperatur-Zeit Kurven für die kinetischen Experimente sind im Anhang in Abbildung .8, .9, .10 auf Seite 108f gezeigt.
} 
Tabelle 5.1: Die Abkühlzeiten in den kinetischen Experimenten. Die Zeit vom Beginn des Abkühlens $t_{0}=0$ bis zum Erreichen der Binodaltemperatur ist $t_{1}$, und $t_{2}$ ist die Zeit von $t_{0}$ bis zum Erreichen des Sollwerts.

\begin{tabular}{rrrrr}
\hline \hline$x_{0}$ & $T_{\text {krit }}\left[{ }^{\circ} \mathrm{C}\right]$ & $T_{\text {soll }}\left[{ }^{\circ} \mathrm{C}\right]$ & $t_{1}[\mathrm{~s}]$ & $t_{2}[\mathrm{~s}]$ \\
\hline \hline 0.23 & 156 & 100 & 32 & 67 \\
0.26 & 165 & 107 & 82 & 517 \\
0.41 & 193 & 100 & 16 & 44 \\
\hline
\end{tabular}

tauchten Satelliten neben den Braggreflexen auf, wie Abbildung 5.1 zeigt. Bei der Zuordnung von Gitterkonstanten zu den Satellitenpositionen ergibt sich, daß die Gitterkonstanten größer bzw. kleiner sind als die von reinem Natriumchlorid bzw. Silberchlorid bei $100{ }^{\circ} \mathrm{C}$. Nach mehr als $10^{3} \mathrm{~s}$ verschiebt sich die Lage der Satelliten analog $\mathrm{zu}$ den früheren Messungen proportional $\mathrm{zu} t^{-1 / 3}$. Zu früheren Zeiten hin deutet sich an, daß die Korrelationslänge einen konstanten Wert annimmt. Dies steht in Übereinstimmung mit den Kleinwinkelmessungen für $x_{0}=0.50$. Auch die gemessenen Korrelationslängen sind konsistent zu den Werten aus den Pulvermessungen. Im Vorgriff auf die Messungen im metastabilen Bereich sind die zwei gemessenen Korrelationslängen für $x_{0}=0.23$ mit eingezeichnet. Eine Aussage über die zeitabhängige Lage des Korrelationsmaximums bei $x_{0}=0.23$ kann anhand der zwei gemessenen Satellitenpositionen nicht getroffen werden. Es wäre jedoch ein ähnliches Verhalten wie bei den Proben mit $50 \%$ und $41 \%$ AgCl-Anteil zu erwarten. Die Lage und Form des Braggreflexes bleiben trotz Entmischung in allen drei Einkristallen über einen Zeitraum von $10^{3} \mathrm{~s}$ unverändert.

Bei der Behandlung des quasistabilen Zustands auf Seite 65 war eine Verbreiterung der Braggreflexe aufgrund von Korngrößeneffekten nicht auszuschließen. Mit Hilfe von Abbildung 5.1 kann eine Korrelationslänge von $1870 \AA$ A nach $8 \mathrm{~d}$ abgeschätzt werden. Bei einem Volumenverhältnis im quasistabilen Zustand von

$$
\frac{V_{2}}{V_{1}}=\frac{x_{0}-x_{1}}{x_{2}-x_{0}}=\frac{0.41-0.05}{0.95-0.41}=\frac{1}{1.5}
$$

ist das Verhältnis der Korndurchmesser bei einer Korrelationslänge von $1870 \AA$

$$
\frac{D_{2}}{D_{1}}=\sqrt[3]{\frac{V_{2}}{V_{1}}}=\frac{870 \AA}{1000 \AA} .
$$

Angesichts der langen Auslagerungszeiten und der nahezu temperaturunabhängigen 


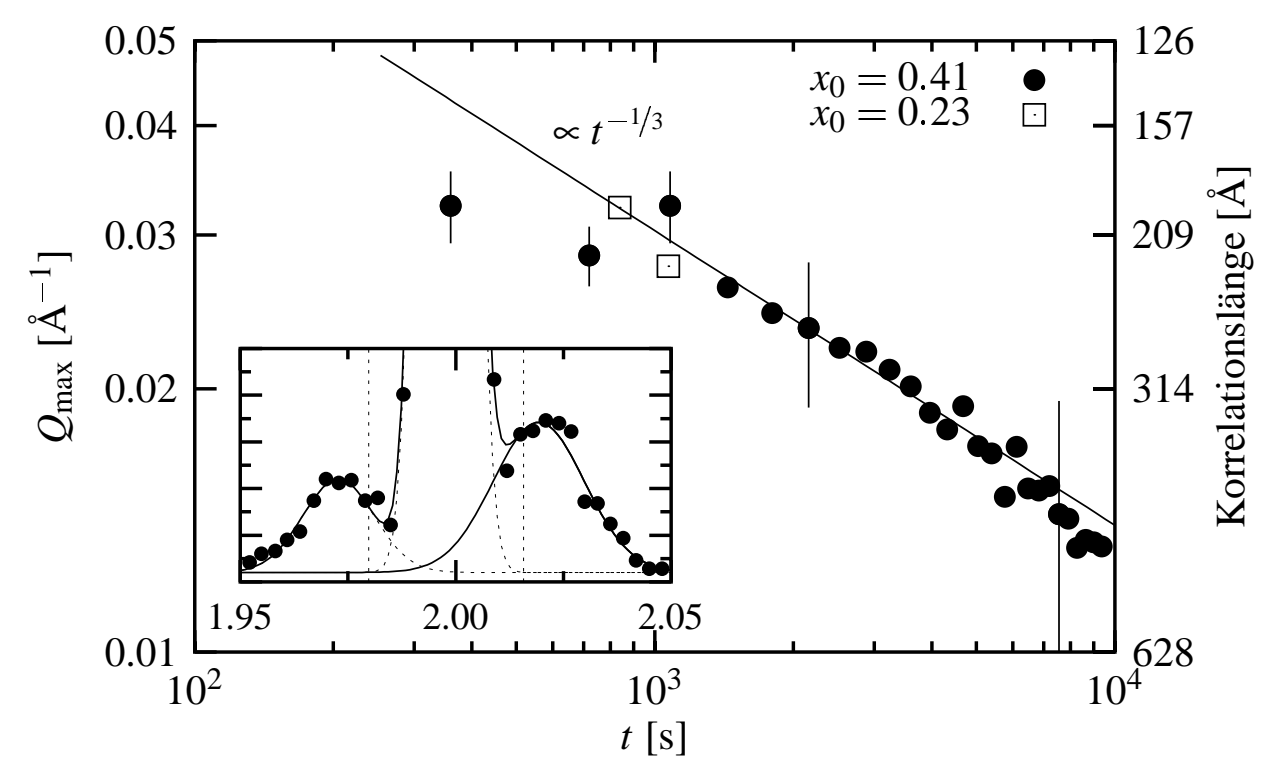

Abbildung 5.1: Die zeitabhängige Lage der Satelliten für $x_{0}=0.41$ und $x_{0}=0.23$ bei $100{ }^{\circ} \mathrm{C}$. Das kleine Bild ist eine Beispielmessung für einen Satellitenscan mit $x_{0}=0.41$ am $(200)$ Reflex nach $1430 \mathrm{~s}$. Die gestrichelten Linien markieren die Reflexpositionen von reinem $\mathrm{AgCl}$ und $\mathrm{NaCl}$ bei $100{ }^{\circ} \mathrm{C}$.

Entmischungskinetik im Bereich von $100-170{ }^{\circ} \mathrm{C}$ ist davon auszugehen, daß diese Korndurchmesser erreicht wurden, selbst wenn die Proben nur bei Raumtemperatur lagerten. Mithin sind merkliche Verbreiterungen der Braggreflexe im quasistabilen Zustand durch Korngrößeneffekte auszuschließen.

Die Entmischung in den Einkristallen durchläuft wie in den SANS-Experimenten die frühen Stadien innerhalb der ersten $10^{2} \mathrm{~s}$. Auf diesen Zeitbereich konzentrieren sich die zeitaufgelösten Experimente. Prinzipiell kann die zeitliche Auflösung $\Delta t$ in den stroboskopischen Experimenten beliebig klein gewählt werden. Jedoch limitiert die begrenzte Meßzeit an den Neutronenquellen die möglichen Zeitkanalbreiten. In der Regel wurde mit $\Delta t=10 \mathrm{~s}$ gemessen. Bei dieser Zeitauflösung und einer Periodendauer von $T=t_{\text {Auslagern }}+t_{\text {Homogenisieren }}=2 \cdot 300 \mathrm{~s}$ dauert eine stroboskopische Messung am IN-12 für eine Temperatur $72 \mathrm{~h}$. Bei $\Delta t=10 \mathrm{~s}$ sind die Änderungen zwischen zwei bis drei aufeinander folgende Spektren so klein, daß die Messungen über jeweils drei Zeitschritte aufaddiert wurden, um die Statistik zusätzlich zu erhöhen: $10 \mathrm{~s}, 20 \mathrm{~s}$ und 30 s ergeben den Zeitschritt 20 s; 20 s, 30 s und 40 s ergeben den Zeitschritt 30 s, u. s.w.

Sind die Spektren der homogenen Phase sehr gut durch eine Lorenzfunktion zu beschreiben, nimmt die Güte dieser Beschreibung nach dem Abschrecken kontinuierlich ab, wie in Abbildung $5.2 \mathrm{zu}$ sehen ist. Um unterscheiden zu können, ob das Spektrum mit einer oder mehreren Lorenzfunktionen $\mathrm{zu}$ beschreiben ist, es sich folglich um ein oder mehrere Phononen handelt, wurden die entsprechenden Anzahl von Lo- 


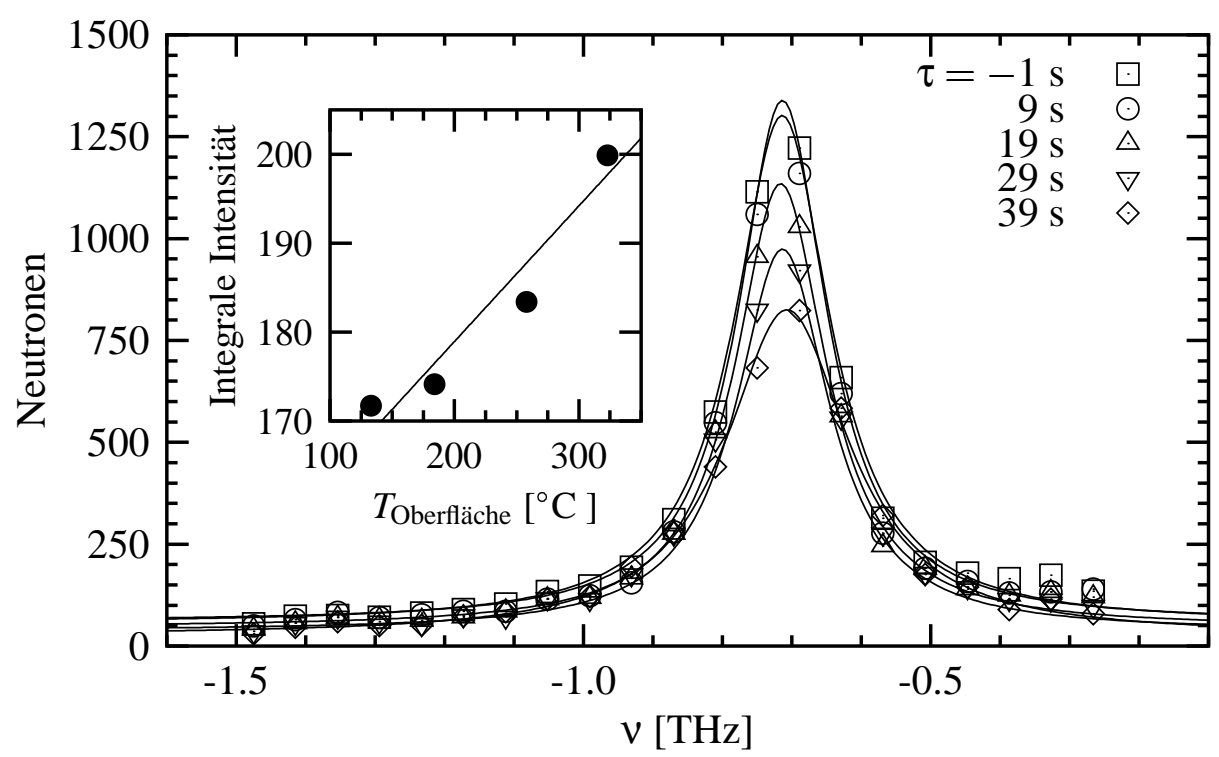

Abbildung 5.2: Die Spektren für $x_{0}=0.41$ während des Abkühlens bei $\tau=-1,19,29$ und $39 \mathrm{~s}$. Mit gezeigt ist maximale Intensität in Abängigkeit von der Temperatur an der Probenoberfläche.

renzfunktionen an die Spektren angepaßt. Anhand der Summe der kleinsten Fehlerquadrate wurde dann die kleinste Anzahl von Signalen ausgewählt, um das Spektrum zu beschreiben. So wurden zunächst alle Spektren mit ein bzw. zwei Lorenzkurven und einem konstantem Untergrund ${ }^{2}$ beschrieben. Während des Abkühlens ändern sich die Lage des Frequenzmaximums $v_{\max }$ und die Kurvenform nicht, während die Halbwertsbreite $\Delta v$ zwischen -1 s und 39 s um $40 \%$ zunimmt. Für die Zeiten $\tau<0$ ist dem Maximum des Spektrums eindeutig die homogene Phase zuzuordnen. Die sich nicht mit dem Unterschreiten der Binodaltemperatur ändernde Lage des Maximums untermauert das Konzept einer initial homogenen Phase im Zweiphasengebiet des Phasendiagramms. Es findet sich ferner keine zunehmende Intensität auf beiden Seiten des Maximums, die im Falle einer Keimbildung im Temperaturbereich zwischen der kohärenten Binodale und der Binodale zu erwarten ist. In der Abbildung ist ergänzend die Integrale Intensität in Abhängigkeit von der Oberflächentemperatur gezeigt. Wie nach Gleichung (1.37) zu erwarten, nimmt die Intensität mit der Temperatur ab.

Eine Auswahl von Spektren für die Messung für $x_{0}=0.41$ bei $100{ }^{\circ} \mathrm{C}$ ist in Abbildung 5.3 abgebildet. Im Laufe der Zeit verschiebt sich das Maximum der Phononenfrequenz von $0.7 \mathrm{THz}$ zu kleineren Frequenzen und die Halbwertsbreite nimmt in den frühen Stadien zu. Gleichzeitig bildet sich ein Nebenmaximum bei 0.9 THz, dessen Lage sich während der frühen Zeiten zu höheren Frequenzen verschiebt. Dem Maximum

\footnotetext{
${ }^{2}$ Um den Untergrund mit mehr als nur einer Konstanten beschreiben zu können, müßten mehr Punkte gemessen werden. In Vorversuchen konnte aber keine signifikante Änderung des Untergrunds außerhalb der gezeigten Frequenzbereiche gefunden werden.
} 

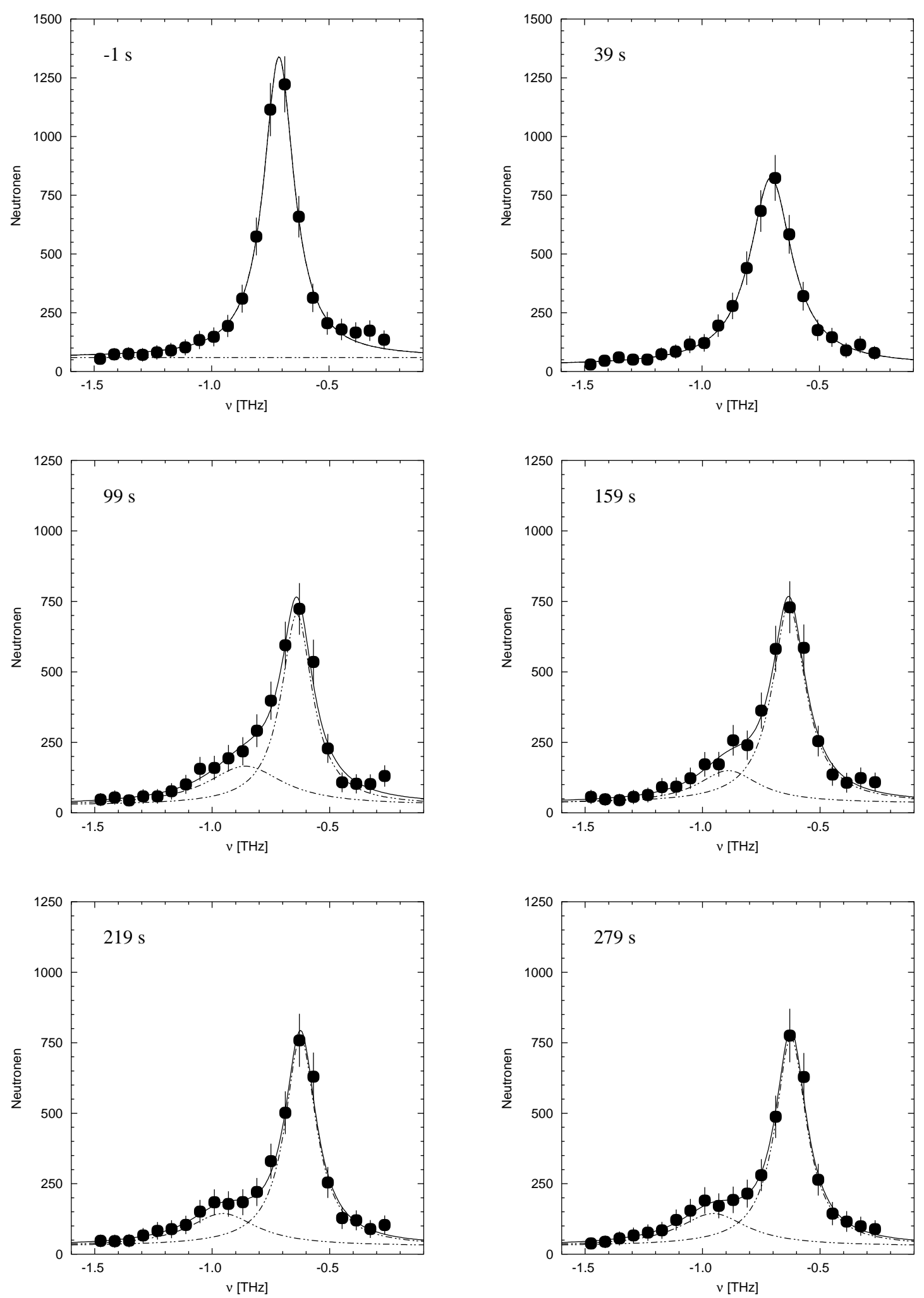

Abbildung 5.3: Die zeitliche Entwicklung des Spektrums bei $Q=(20.30)$ und $100{ }^{\circ} \mathrm{C}$. Es sind die Spektren zeilenweise nach $\tau=-1,39,99,159,219$ und 279 s gezeigt. Es ist zu beachten, daß sich die Skalierung der Ordinate nach $39 \mathrm{~s}$ ändert. 


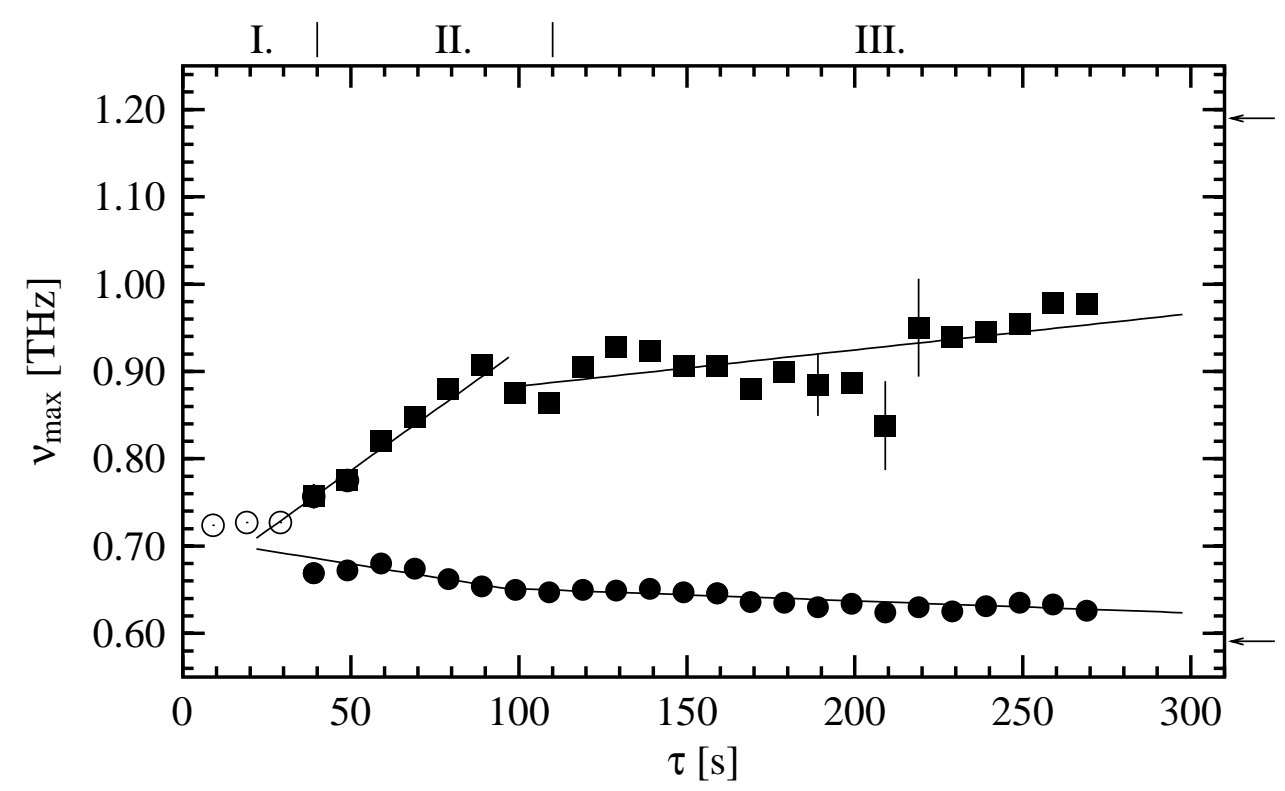

Abbildung 5.4: Die Frequenzaufspaltung des TA Phonons bei (2 0.3 0) für $x_{0}=0.41$ bei $100{ }^{\circ} \mathrm{C}$

bei $\tau=-1 \mathrm{~s}$ ist zunächst die homogene Phase und zu späteren Zeiten die silberreiche Phase und die evtl. verbleibende homogene Phase zuzuordnen. Das Nebenmaximum, dessen Zentrum sich im Verlauf der Entmischung zu höheren Frequenzen verschiebt, gehört zur Na-reichen Phase. Die Frequenz der homogenen Phase $v_{0}=0.73 \mathrm{THz}$ entspricht der mittleren Zusammensetzung $x_{0}=0.41$. Die Zusammensetzung stimmt gut mit der Frequenz überein, die für $0.73 \mathrm{THz}$ aus Abbildung 4.4 abzulesen ist, wenn die Frequenzverschiebung aufgrund der unterschiedlichen Temperaturen berücksichtigt wird. Es wird dabei vorausgesetzt, daß die Abhängigkeit der Frequenz von der Zusammensetzung, wie sie im homogenen Bereich des Phasendiagramms gemessen wurde, im Zweiphasengebiet gültig ist. Nach $300 \mathrm{~s}$ können analog den Frequenzen der beiden Phononen die Konzentrationen $x_{1}^{\prime}=0.2$ und $x_{2}^{\prime}=0.8$ zugeordnet werden. Am Ende der frühen Entmischungsstadien zeigen die inelastischen Neutronenstreuexperimente und die SANS-Experimente die gleiche chemischen Entmischung.

Es ist schon mit bloßem Auge zu erkennen, daß die Verschiebung des Phonons der natriumreichen Phase deutlich größer ist, als die des Phonons der silberreichen Phase. Die Lage der Intensitätsmaxima in Abhängigkeit von der Zeit zeigt Abbildung $5.4^{3}$. Um die Spektren quantifizieren zu können, wurden sowohl Lorenzfunktionen als auch Streufunktionen für einen gedämpften harmonischen Oszillatoren gemäß (1.37) an die Daten angepaßt. Die aus den beiden Anpassungen erhaltenen Parametersätze unterscheiden sich innerhalb der Fehlergrenzen nicht. Zeitlich sind drei Abschnitte unter-

\footnotetext{
${ }^{3}$ In den folgenden Abbildungen sind, wenn nicht anderes gekennzeichnet, die Parameter der homogenen Phasen mit $\odot$, die natriumreiche Phase mit $\square$ und die silberreiche Phase mit $\bullet$ gekennzeichnet.
} 
scheidbar: Die ersten drei Meßpunkte bis 40 s sind der homogenen Phase zuzuordnen, wobei die Solltemperatur von $100{ }^{\circ} \mathrm{C}$ bereits nach $\tau=28 \mathrm{~s}$ erreicht ist. Bis $40 \mathrm{~s}$ wirkt sich die Entmischung nicht auf die Lage des Maximums im Spektrum aus. Im zweiten Abschnitt von 40-110 s verschieben sich $v_{\max }$ für beide Phononen deutlich. Die Frequenzverschiebungen der Phononen betragen $0.18 \mathrm{THz}$ und $-0.08 \mathrm{THz}$ bzw. $\partial v_{1} / \partial \tau=(+2.8 \pm 0.5) \cdot 10^{-3} \mathrm{THz} / \mathrm{s}$ und $\partial v_{2} / \partial \tau=(-0.6 \pm 0.1) \cdot 10^{-3} \mathrm{THz} / \mathrm{s}$. Nach mehr als $110 \mathrm{~s}$ verschieben sich die Phononenfrequenzen mit $\partial v_{1} / \partial \tau=(+0.4 \pm 0.1) \cdot 10^{-3} \mathrm{THz} / \mathrm{s}$ und $\partial v_{2} / \partial \tau=(-0.14 \pm 0.02) \cdot 10^{-3} \mathrm{THz} / \mathrm{s}$ weiter. Diese unterschiedlichen Verschiebungen für die beiden Phasen sind mit der Konzentrationsabhängigkeit der Frequenz $v(x)$ einfach zu verstehen: In dem Konzentrationsbereich $x_{0} \rightarrow x_{1}^{\prime}$, den die natriumreiche Phase während der Entmischung durchläuft, ist die Abhängigkeit der Frequenz von der Zusammensetzung $\partial v_{1} / \partial x$ größer als in dem Konzentrationsbereich $x_{0} \rightarrow x_{2}^{\prime}$, den die silberreiche Phase durchläuft. Im dritten Abschnitt ist die Verschiebung der Phononen im Bereich von $0.05 \mathrm{THz}$ bzw. weniger als $0.01 \mathrm{THz}$. Eine genauere Angabe ist bei diesen kleinen Änderungen aufgrund der Fehler nicht möglich. Es muß aber die Entmischung, wenn auch deutlich langsamer, weiter voranschreiten. Am rechten Rand von Abbildung 5.4 sind die Frequenzen des quasistabilen Zustands mit zwei Pfeilen markiert. Wie leicht zu erkennen ist, ist insbesondere die $N a$-reiche Phase noch weit vom quasistabilen Zustand entfernt.

Mit der Änderung der lokalen Zusammensetzung geht also tatsächlich die Frequenzänderungen einher. Aus den SANS-Experimenten [Caspary u. a. 2001] ist bekannt, daß frühe Entmischung auf einer Zeitskala von $10^{2} \mathrm{~s}$ stattfindet, genau in dem Zeitfenster, in dem die Phononenspektren ihre größten Änderungen zeigen. Die Frequenzänderung spiegelt also die lokale chemische Entmischung wider. Die Zeitskala aus den SANSExperimenten kann nun anhand der Phononenspektren verfeinert werden. In den ersten $40 \mathrm{~s}$ kann zunächst bis auf die Intensitätsabnahme keine Änderung im Spektrum gefunden werden. Die Halbwertsbreite der Phononen bleibt in diesem Zeitraum nahezu unverändert. Nach ca. $40 \mathrm{~s}$ setzt dann eine schnelle Frequenzaufspaltung ein, die bis $110 \mathrm{~s}$ andauert. Dies ist genau der Zeitraum, in dem die chemische Entmischung bis zur kohärenten Spinodale erfolgt, wie die inelastischen Neutronenstreuexperimente und die Integrale Intensität $\tilde{Q}$ aus den Kleinwinkelexperimenten, vgl. Abbildung 2.3, übereinstimmend zeigen. Nur konnte in Kleinwinkelexperimenten der Anstieg von $\tilde{Q}$ lediglich mit einem einzigen Meßpunkt verfolgt werden. Des Weiteren findet sich das Abknicken der Frequenzverschiebung bei $100 \mathrm{~s}$ in der Integralen Intensität der SANSExperimente wieder. Abbildung 5.4 zeigt mit der kontinuierlichen Verschiebung der Frequenzen innerhalb der ersten $100 \mathrm{~s}$, daß eine kontinuierliche Änderung der lokalen Konzentration erfolgt, und eine kontinuierliche Änderung der Zusammensetzung bedeutet einen spinodalen Entmischungsmechanismus! Nach mehr als $110 \mathrm{~s}$ nimmt die Frequenzaufspaltung mit der fortschreitenden Entmischung weiter zu. Im Anschluß an die schnelle Entmischung nahm in den S ANS-Experimenten auch die Integrale Inten- 

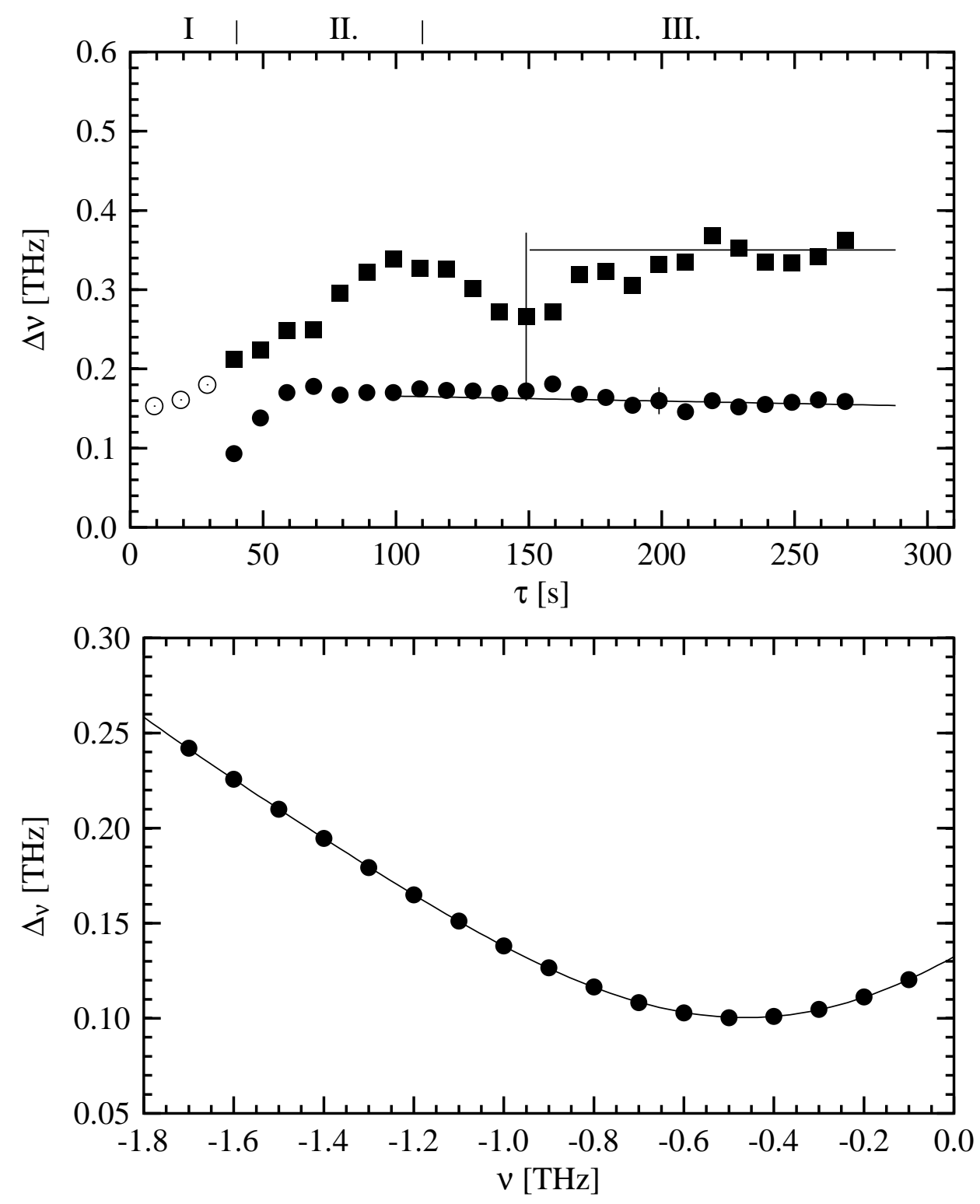

Abbildung 5.5: Die zeitabhängigen Halbwertsbreiten $\Delta v$ der angepaßten Lorenzfunktionen und die frequenzabhängige Geräteauflösung $\Delta_{v}$ für $x_{0}=0.41$

sität zwar schwach, aber kontinuierlich weiter zu. Da zu diesem Zeitpunkt das Gitter nur schwach relaxiert, und dies ebenso einen Einfluß auf die Phononenfrequenzen hat — vgl. Gleichung (4.16) — ist die fortschreitende Aufspaltung der Frequenz auf einen zunehmenden Entmischungsgrad und die Relaxation des Gitters zurückzuführen.

In Abbildung 5.5 sind die experimentellen Halbwertsbreiten und die Auflösungshalbwertsbreiten widergegeben. Im ersten Zeitbereich bis $40 \mathrm{~s}$ verbreitert sich das Phonon der homogenen Phase kontinuierlich. Im zweiten Abschnitt nimmt $\Delta v_{1}$ stark zu. Ob die Halbwertsbreite im dritten Abschnitt weiter zunimmt oder konstant bleibt, kann 


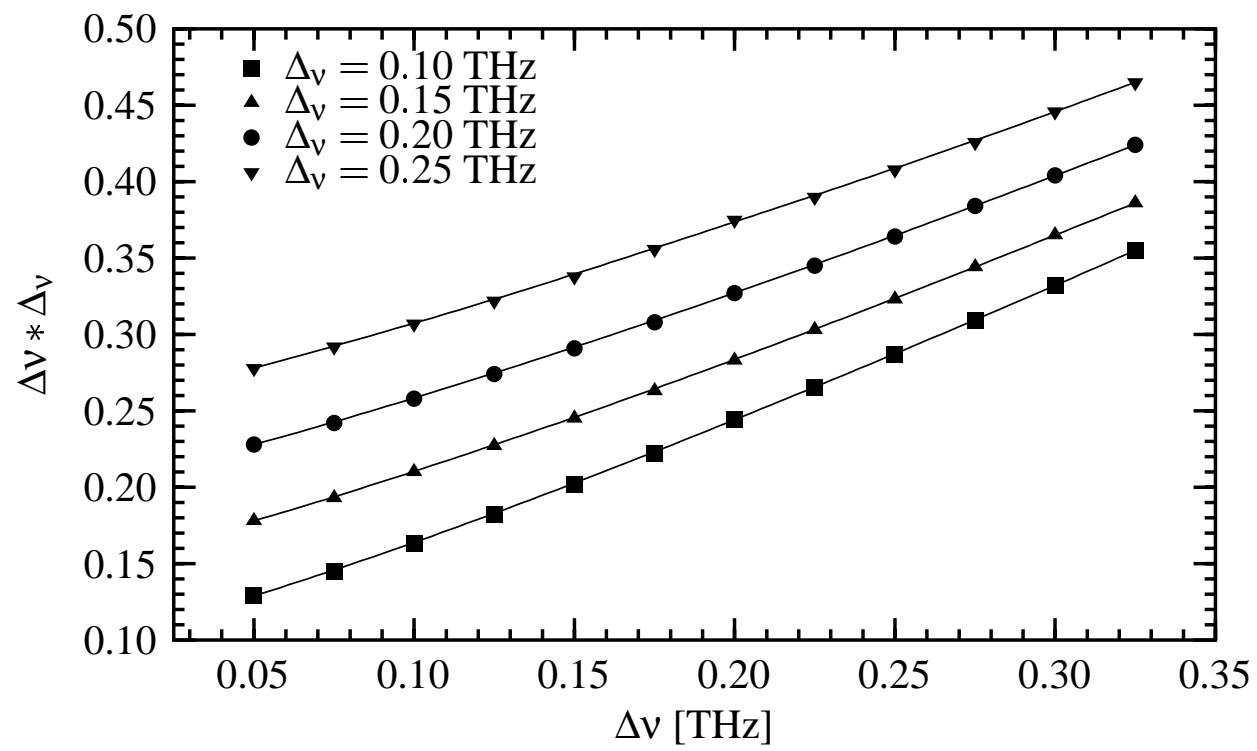

Abbildung 5.6: Zur Entfaltung Halbwertsbreite eines Signals $\Delta v * \Delta_{v}$, das durch Faltung einer Lorenzkurve mit einer Halbwertsbreite $\Delta v$ mit einer Gaußfunktion mit der Breite $\Delta_{v}$ entstanden ist

bei der Streuung und den Fehlern der angepaßten Halbwertsbreite nicht festgelegt werden. Im Gegensatz hierzu ist $\Delta v_{2}$ nahezu über den gesamten beobachteten Zeitraum konstant. Die Halbwertsbreiten im dritten Abschnitt sind im Mittel $\Delta v_{1}=0.35 \mathrm{THz}$ und $\Delta v_{2}=0.16 \mathrm{THz}$. Dem Gegenüber stehen die Halbwertsbreiten $\Delta v_{1}=0.18 \mathrm{THz}$ und $\Delta v_{2}=0.14 \mathrm{THz}$ im quasistabilen Zustand. Während $\Delta v_{2}$ nahezu der Breite im quasistabilen Zustand entspricht, ist $\Delta v_{1}$ ca. um einen Faktor 1.9 breiter. Ein ähnliches Verhältnis zeigt sich ferner im Vergleich mit den Auflösungshalbwertsbreiten $\Delta_{v_{1}}=0.13 \mathrm{THz}$ und $\Delta_{\mathrm{v}_{2}}=0.11 \mathrm{THz}$. Für beide Phasen sind die Halbwertsbreiten gröBer als die auflösungsbedingten Halbwertsbreiten. Bei der Entfaltung von $\Delta v$ mit der Auflösung $\Delta_{v}$, vgl. Abbildung 5.6, ergeben sich Halbwertsbreiten von $\Delta v_{1}=0.30 \mathrm{THz}$ und $\Delta v_{2}=0.11 \mathrm{THz}$, die gleiche Verhältnisse zeigen.

Die Verbreiterung von $\Delta v$ kann insbesondere zwischen 40-100 s zwei Ursachen habe: Zum einen kann sie in einer Konzentrationsverteilung begründet sein, oder sie kann durch eine zunehmende Dämpfung zustande kommen. Bei einer Dämpfung als Ursache ist zu beachten, daß die anharmonischere silberreiche Phase nur eine geringe Verbreiterung zeigt. Die starke Verbreiterung der natriumreichen Phase wird also durch Konzentrationsgradienten hervorgerufen. Eine merkliche Verbreiterung der natriumreichen Phase durch restliche homogene Phase ist auszuschließen, wenn die Integrale Intensität nach dem Erreichen von $100{ }^{\circ} \mathrm{C}$ nach $\tau=28 \mathrm{~s}$ mit der Integralen Intensität nach $279 \mathrm{~s}$ 
verglichen werden:

$$
\begin{aligned}
& \tilde{I}(28 \mathrm{~s})=I_{\max , 0} \cdot \Delta v_{0} \cdot v_{0}^{2}=86 \\
& \tilde{I}(279 \mathrm{~s})=I_{\max , 1} \cdot \Delta \mathrm{v}_{1} \cdot v_{1}^{2}+I_{\max , 2} \cdot \Delta v_{2} \cdot v_{2}^{2}=85
\end{aligned}
$$

Es findet sich alle Intensität in den entmischten Phasen wieder, und es bedeutet das die gesamte Probe entmischt. Für die mittels SANS bestimmte kohärente Spinodale bedeutet das, daß die Annahme eines Zweiphasenmodells richtig war.

\subsection{Entmischung im natriumreichen Kristall}

Die gleichen Experimente wie mit der $41 \%$-Probe wurden auch mit $x_{0}=0.23$ durchgeführt. Wie in Abbildung 5.1 bereits gezeigt, konnten auch bei $x_{0}=0.23$ Satelliten nachgewiesen werden. Das Auftreten von Satelliten weist auf ein langreichweitige Korrelation in der Probe während der Entmischung hin. In Abbildung 5.7 sind repräsentative Spektren während der Entmischung gezeigt. Wie schon bei $x_{0}=0.41$ gibt es einen Zeitbereich, in dem sich die Lage und die Form des Spektrums nur gering ändern, was insbesondere für $\tau<0$ gilt. Mit dem Unterschreiten der Binodaltemperatur ist nur eine geringe Verschiebung der maximalen Phononenfrequenz von $v_{\max } \approx 0.9 \rightarrow 1.0 \mathrm{THz}$ zu beobachten. Nach mehr als $60 \mathrm{~s}$ findet sich ein Nebenmaximum, dessen Lage sich von $v_{\max } \approx 0.8 \rightarrow 0.7 \mathrm{THz}$ verschiebt. Dem Maximum ist die $\mathrm{Na}$-reiche Phase und dem Nebenmaximum die $A g$-reiche Phase zuzuordnen. Wie schon mit dem Auge zu erkennen, ist bei 93 s die Halbwertsbreite sehr schmal. Die kleinen Halbwertsbreiten und die vergleichsweise geringe Intensität stellen bei der Anpassung einer Streufunktion für die silberreiche Phase an die Spektren ein Problem dar: Bei den geringen Abweichungen von einem ein-Phonon-Spektrum streuen die Parameter für das Nebenmaximums sehr stark, so daß sie mit Bedacht zu bewerten sind.

$v_{\max }(t)$ ist für beide Maxima in Abbildung 5.8 widergegeben. Wie schon an den Einzelspektren zu erkennen ist, verschiebt sich die Lage der Maxima nur sehr gering. Die initiale homogene Phase geht kontinuierlich in die natriumreiche Phase über, weil sich die Zusammensetzungen von homogener und natriumreicher Phase nur geringfügig unterscheiden. Während sich in der $41 \%$-Probe die Frequenz der homogenen Phase nicht verschiebt, findet sich hier eine merkliche Frequenzverschiebung von mehr als $0.03 \mathrm{THz}$ statt. Es ist somit davon auszugehen, daß die Entmischung bereits vor $60 \mathrm{~s}$ einsetzt. Die Ausbildung der silberreichen Phase kann zu diesen frühen Zeiten nur nicht aufgelöst werden, so daß Spektren gemessen werden, das als ein-Phonon-Spektren interpretiert werden können.

Nach mehr als $60 \mathrm{~s}$ sind die linearisierten Frequenzverschiebungen $\partial v_{1} / \partial \tau=(0.24 \pm$ $0.03) \cdot 10^{-3} \mathrm{THz} / \mathrm{s}$ und $\partial v_{2} / \partial \tau=(-0.28 \pm 0.07) \cdot 10^{-3} \mathrm{THz} / \mathrm{s}$. Wird die zeitliche Entwicklung von $v_{\max }(t)$ mit der aus der $41 \%$-Probe verglichen, fällt sofort auf, daß nach 

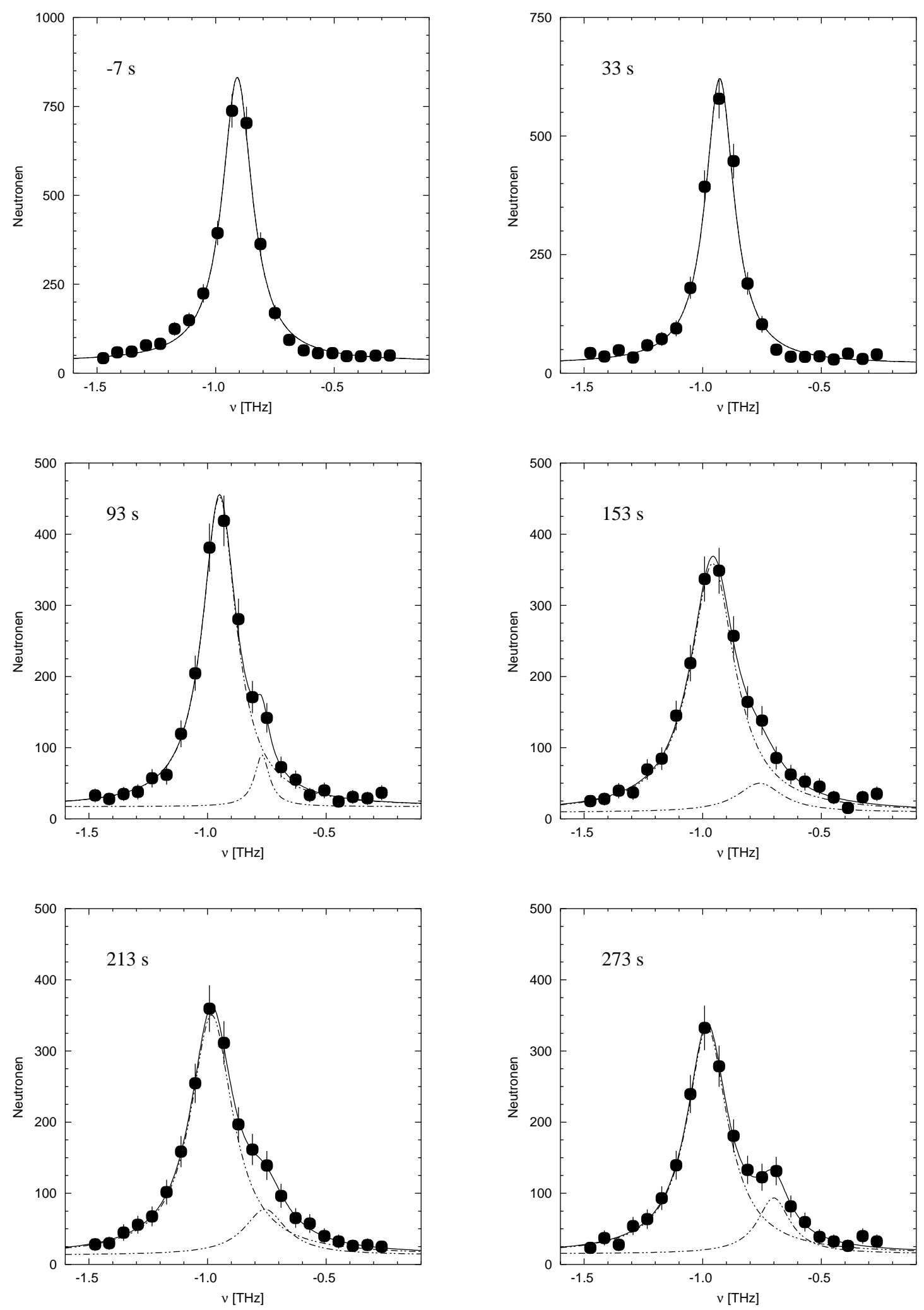

Abbildung 5.7: Die zeitliche Entwicklung des Spektrums für $x_{0}=0.23$ bei $Q=(20.30)$ und $100{ }^{\circ} \mathrm{C}$. Es sind die Spektren zeilenweise nach $\tau=-7,33,93,153,213$ und 273 s gezeigt. Es ist zu beachten, daß sich die Skalierung der Intensität ändert. 


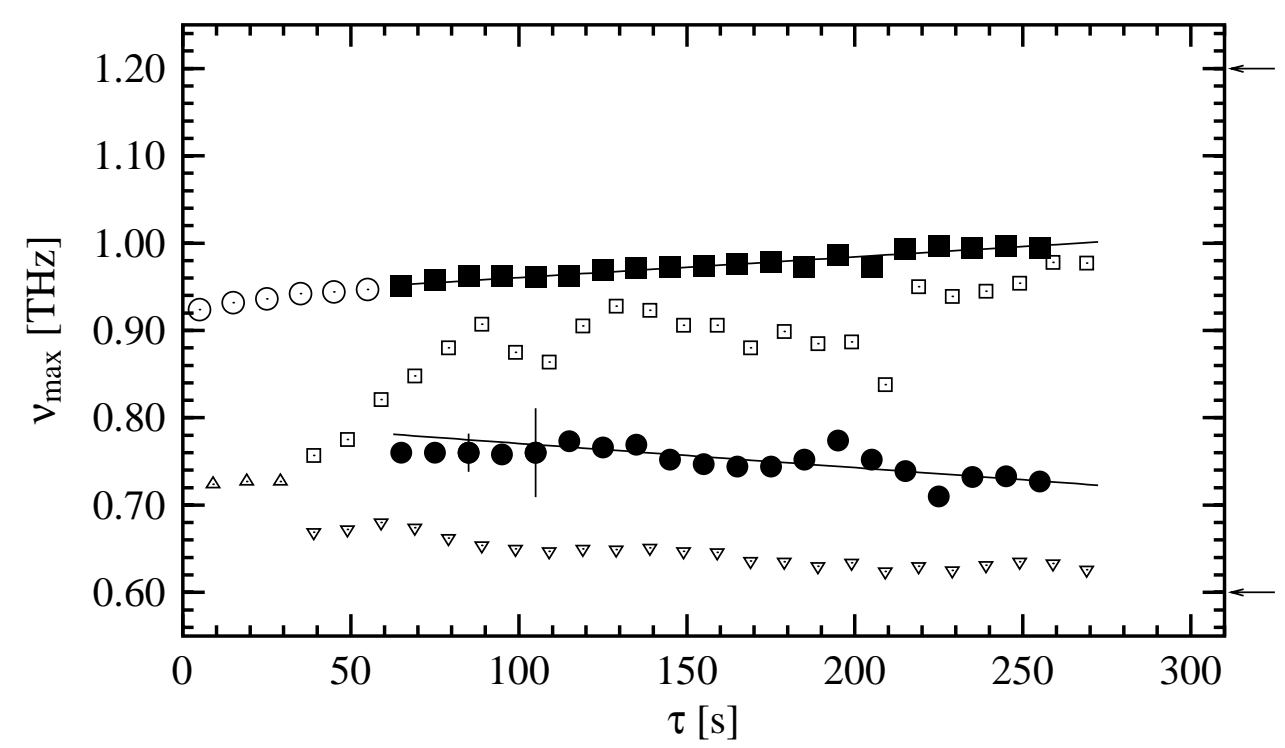

Abbildung 5.8: Die Frequenzaufspaltung des TA Phonons bei $(20.30)$ für $x_{0}=0.23$ bei $100{ }^{\circ} \mathrm{C}$. Die Phononenfrequenzen $v_{0}, v_{1}$ und $v_{2}$ sind als $\odot$, $\bullet$ und $\boldsymbol{a u f g e t r a g e n . ~ Z u m ~ V e r g l e i c h ~ s i n d ~}$ auch die Frequenzen $v_{0}^{x_{0}=0.41}(\diamond), v_{1}^{x_{0}=0.41}(\odot)$ und $v_{2}^{x_{0}=0.41}(\nabla)$ von dem kinetischen Experimenten für eine mittlere von 0.41 mit eingezeichnet. Am rechten Rand sind die Frequenzen für den entmischten Zustand mit Pfeilen markiert.

mehr als100 s die Frequenzen der natriumreichen Phase sich zusehends der Frequenz der natriumreichen Phase $v_{1}=1 \mathrm{THz}$ aus der Probe mit $x_{0}=0.41$ annähert. Die Phononenfrequenz der silberreichen Phase ist aber um $0.1 \mathrm{THz}$ gegenüber der $41 \%$-Probe verschoben. Beide Frequenzen sind nach 270 s noch weit von den Frequenzen im quasistabilen Zustand entfernt, die mit Pfeilen am rechten Rand von Abbildung 5.8 markiert sind. Das Erreichen der gleichen Frequenz und damit Konzentration für die natriumreiche Phase spricht für den spinodalen Entmischungsmechanismus in der $23 \%$-Probe. Die Konzentrationsänderungen $x_{0}-x_{1}^{\prime}=0.03$ sind zu gering um eine große kontinuierliche Frequenzaufspaltung zu messen. Stattdessen geht die homogene Phase mit kleinen Frequenzänderungen in die natriumreiche Phase über, während die silberreiche Phase erst nach mehr als 60 s detektiert werden kann. Die eigentliche Verschiebung der Frequenz der silberreichen Phase erfolgte jedoch schon vorher. Dies ist zudem konsistent zur zeitabhängigen Entwicklung der Halbwertsbreite der homogenen Phase. Wie aus Abbildung 5.9 abzulesen ist, nimmt $\Delta v_{0}$ bereits nach mehr als $40 \mathrm{~s} \mathrm{zu}$. Die Entmischung setzt folglich spätestens nach $40 \mathrm{~s}$ ein. Ob es sich bei den $40 \mathrm{~s}$ Latenzzeit um eine zufällige Übereinstimmung zwischen den beiden Proben oder eine Systematik handelt, kann anhand der beiden Experimente nicht abgeleitet werden.

Die Frequenz des TA Phonons in der natriumreiche Phase, die die Majoritätsphase ist, geht gegen den gleichen Wert wie im Kristall mit $x_{0}=0.41$. Folglich muß dann 
auch die Konzentration $x_{1}^{\prime}$ in beiden Phase annähernd gleich sein. Sie liegt mit $x_{1}^{\prime} \approx 0.2$ im Bereich der kohärenten Spinodalen. Die korrespondierende Konzentration für die silberreiche Phase wäre $x_{2} \approx 0.8$ mit der dazugehörigen Frequenz von ca. $0.6 \mathrm{THz}$. Die Frequenz der Ag-reichen Phase liegt nach $250 \mathrm{~s}$ noch oberhalb der Frequenz der homogenen Phase mit $x_{0}=0.41$, und hätte dann eine Konzentration, die kleiner als 0.41 ist. Vielmehr ist davon auszugehen, daß die Phononenfrequenz in der silberreichen Phase nicht mehr die Konzentration in der silberreichen Phase widerspiegelt, sondern vom Ausscheidungsvolumen und der umgebenden natriumreichen Phase abhängig ist. Der Fall, daß die silberreiche Phase tatsächlich eine Zusammensetzung von ca. 0.4 hat ist unwahrscheinlich. In diesem Fall wäre der Volumenanteil der silberreichen Phase bei $15 \%$ und ein besser definiertes Spektrum ist zu erwarten.

Wenn von den Zusammensetzungen der kohärenten Binodale ausgegangen wird, ist das Volumenverhältnis nach $270 \mathrm{~s}$ von silber- zu natriumreicher Phase $V_{2} / V_{1}=1 / 19$. Mit einem mittleren Abstand von $200 \AA$ Å nach 300 s, vgl. Abbildung 5.1, kann das Verhältnis der mittleren Durchmesser von silber- und natriumreicher Bereiche auf

$$
\frac{D_{2}}{D_{1}} \approx \sqrt[3]{\frac{V_{2}}{V_{1}}}=\frac{1}{2.67}=\frac{55 \AA}{145 \AA}
$$

abgeschätzt werden. Der Durchmesser der silberreichen Phase liegt in der Größe einer mittleren freien Weglänge $\ell=50 \AA$ eines Phonons, wie sie auf Seite 30 berechnet wurde, und der Wellenlänge $\lambda \approx 19 \AA$ des Phonons. Die Verschiebung des zur silberreichen Phase gehörenden Phonons zu höheren Frequenzen, deutet auf eine Mittelung der Phononenfrequenz über die silberreiche Phase und die umgebende natriumreiche Phase hin. Das Volumen, über das dabei gemittelt wird, liegt in der Größenordnung $\ell^{3}$. Diese Mittelung hat aber keinen außergewöhnlichen Einfluß auf die Halbwertsbreite ${ }^{4}$ der Phononen. Für die Halbwertsbreite der natriumreiche Phase, siehe Abbildung 5.9, findet sich im Vergleich mit der $41 \%$-Probe keine erhöhte Dämpfung. Sie ist sogar aufgrund der kleineren Konzentrationsdifferenz $x_{0}-x_{1}^{\prime}$ um den Faktor 1.6 schmaler. Es ist auch unwahrscheinlich, daß in dem großen Volumen der natriumreichen Phase ein Phonon auf einen silberreichen Bereich trifft. In der silberreichen Phase sollten die Phononen aber in der Mehrzahl einen Übergang zur natriumreichen Phase treffen. Aber auch die silberreiche Phase zeigt keine übermäßige Verbreiterung, die mit einer Dämpfung gleichzusetzten wäre.

Um das Frequenzverhalten zu parametrisieren, soll von einem kugelförmigen silberreichen Bereich mit dem Radius $R_{2}$ und der Zusammensetzung $x_{2}=0.2$ in einer natriumreichen Umgebung der Konzentration $x_{1}=0.8$ ausgegangen werden. Da die Frequenzabhängigkeit von der Zusammensetzung für natriumreiche Zusammensetzungen größer als für die silberreiche Phase ist, kann das Konzentrationsprofil durch einen

\footnotetext{
${ }^{4}$ Es findet sich die Auftragung der Geräteauflösung gegen die Frequenz in Abbildung .11 im Anhang.
} 


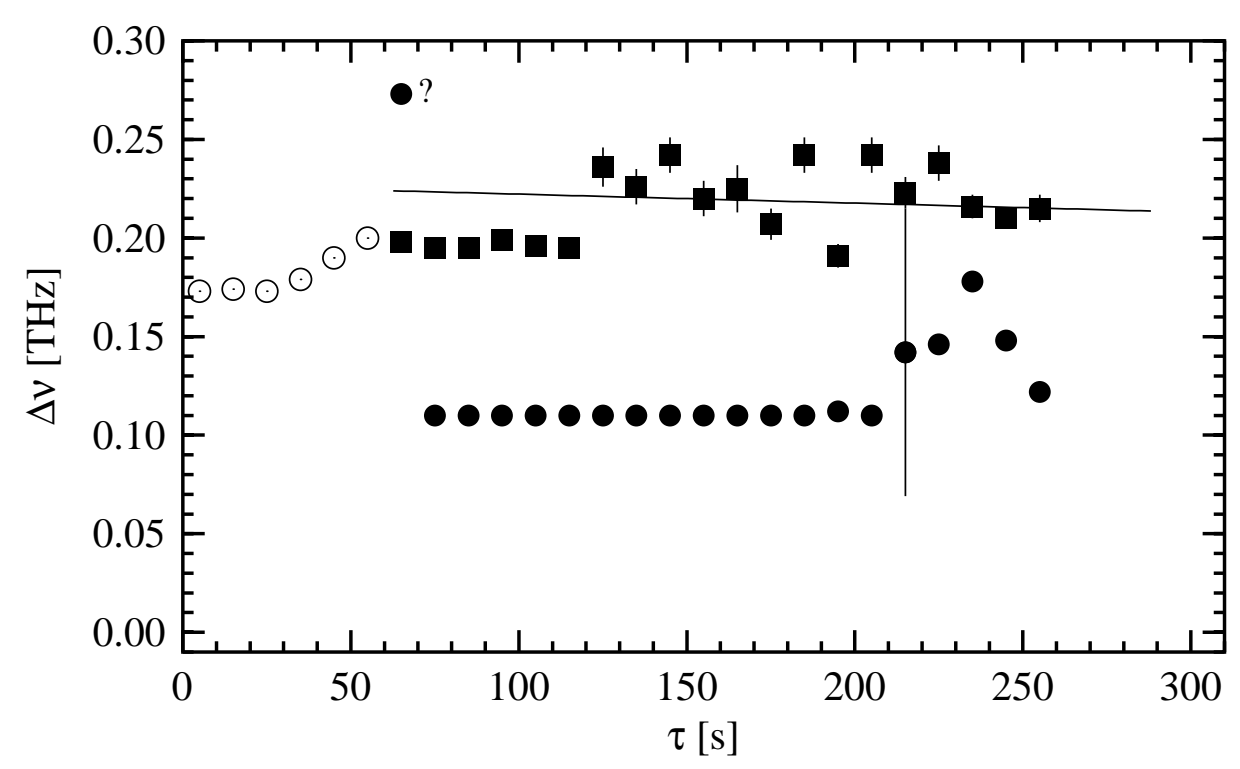

Abbildung 5.9: Die zeitabhängigen Halbwertsbreiten der angepaßten Streufunktion für einen gedämpften harmonischen Oszillator für $x_{0}=0.23$ bei $100{ }^{\circ} \mathrm{C}$

diskontinuierlichen Verlauf vereinfacht werden. Betrachtet wird ein Kugelvolumen mit dem normierten Gesammtradius $R_{0}=1 . R_{2}$ wird so gewählt, daß der Volumenanteil von $V_{2}=4 \pi / 3 R_{2}^{3}$ am Gesamtvolumen $V_{0}=4 \pi / 3$ gleich dem Volumenanteil $\alpha$ aus dem Hebelgesetz der Phasenmengen entspricht. Das Konzentrationsprofil ist dann

$$
x(r)=\left\{\begin{array}{ll}
x_{2} & \text { wenn } r>0 \quad \text { und } r \leq \sqrt[3]{\alpha} \\
x_{1} & \text { wenn } r>\sqrt[3]{\alpha} \text { und } r \leq 1
\end{array} .\right.
$$

Mit Gleichung (4.12) kann das Konzentrationsprofil in ein Frequenzprofil v $(x(r))$ umgerechnet und aus dem Frequenzprofil eine mittlere Frequenz

$$
\langle v\rangle=\int_{0}^{1} v(x(r)) \mathrm{d} r
$$

berechnet werden. Das Ergebnis ist in Abbildung 5.10 gezeigt. Die mittlere Frequenz $\langle v\rangle$ nimmt dem zunehmenden Volumenanteil ab. Bei einem Volumenanteil von $5 \%$ ergibt sich eine mittlere Frequenz von $\langle v\rangle=0.80 \mathrm{THz}$, die mäßig gut mit dem experimentellen Wert von $0.74 \mathrm{THz}$ übereinstimmt. Wenn die Phononen sich aus der silberreichen Phase in die natriumreiche Phase ausbreiten, muß auch der umgekehrte Fall möglich sein, und die Integrale Intensitäten der silberreichen Phase muß erhöht sein. Das Verhältnis der Integralen Intensität nach 60 s zur Integralen Intensität am Ende der 


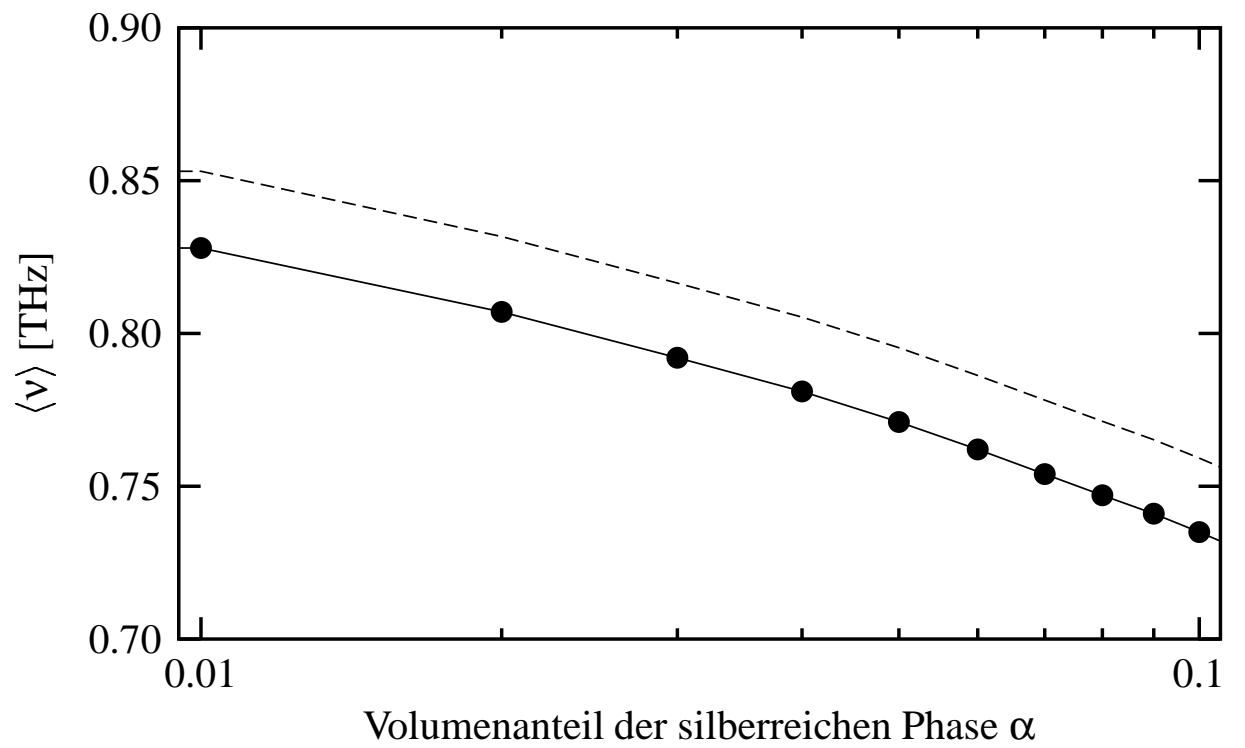

Abbildung 5.10: Die Abhängigkeit der mittleren Phononenfrequenz $\langle v\rangle$ vom Volumenanteil $\alpha$ in halblogarithmischer Auftragung für den kugelsymmetrischen Fall. Die Punkte sind Ergebnisse nach Gleichung (5.1). Da die Werte für $\langle v\rangle$ für $400{ }^{\circ} \mathrm{C}$ berechnet sind, ist der extrapolierte Verlauf für $\langle v\rangle$ bei $100{ }^{\circ} \mathrm{C}$ als gestrichelte Linie mit eingezeichnet.

Meßperiode ist 74/72. Bei einer Entmischung in die Zusammensetzungen der kohärenten Spinodalen ist demzufolge ein Verhältnis von $\tilde{I}_{1} / \tilde{I}_{2}=12 / 1$ abzuschätzen. Es ist das experimentelle Verhältnis aber $\tilde{I}_{1} / \tilde{I}_{2}=5.5 / 1$. Die Integrale Intensität des zur silberreichen Phase gehörenden Phonons ist wirklich erhöht. Aus der zeitabhängigen Frequenzverschiebung in der silberreichen Phase läßt sich ein weiterer Hinweis auf die Ausbreitung von Phononen zwischen den beiden Phasen ableiten. Die Frequenzverschiebung in der $23 \%$-Probe in den mittleren Stadien ist, wenn auch von den Fehlern der Verschiebung kaum gesichert, größer als in der $41 \%$-Probe. Das ist auf den Einfluß der Oswaldreifung zurückzuführen, der sich bei kleinen Volumenanteilen bemerkbar macht, wenn davon auszugehen ist, daß die Entmischunggeschwindigkeit in den mittleren Entmischungsstadien und die Relaxation des Gitters in beiden Proben vergleichbar sind. Die Aussage, daß die Phononen die lokale Konzentration wiederspiegeln muß demzufolge auf hinreichend große Bereiche eingeschränkt werden.

Die Entmischung bei $x_{0}=0.23$ erfolgt analog zur $41 \%$-Probe nach dem spinodalen Mechanismus und liefert bezüglich des Entmischungsmechanismus keine neuen Erkenntnisse. Es findet sich jedoch ein Einfluß des Volumenanteils einer Phase und dem Konzentrationsprofil auf deren Phononenfrequenz. Die experimentellen Ergebnisse deuten darauf hin, daß die Phononen räumlich über das Konzentrationsprofil mitteln. 


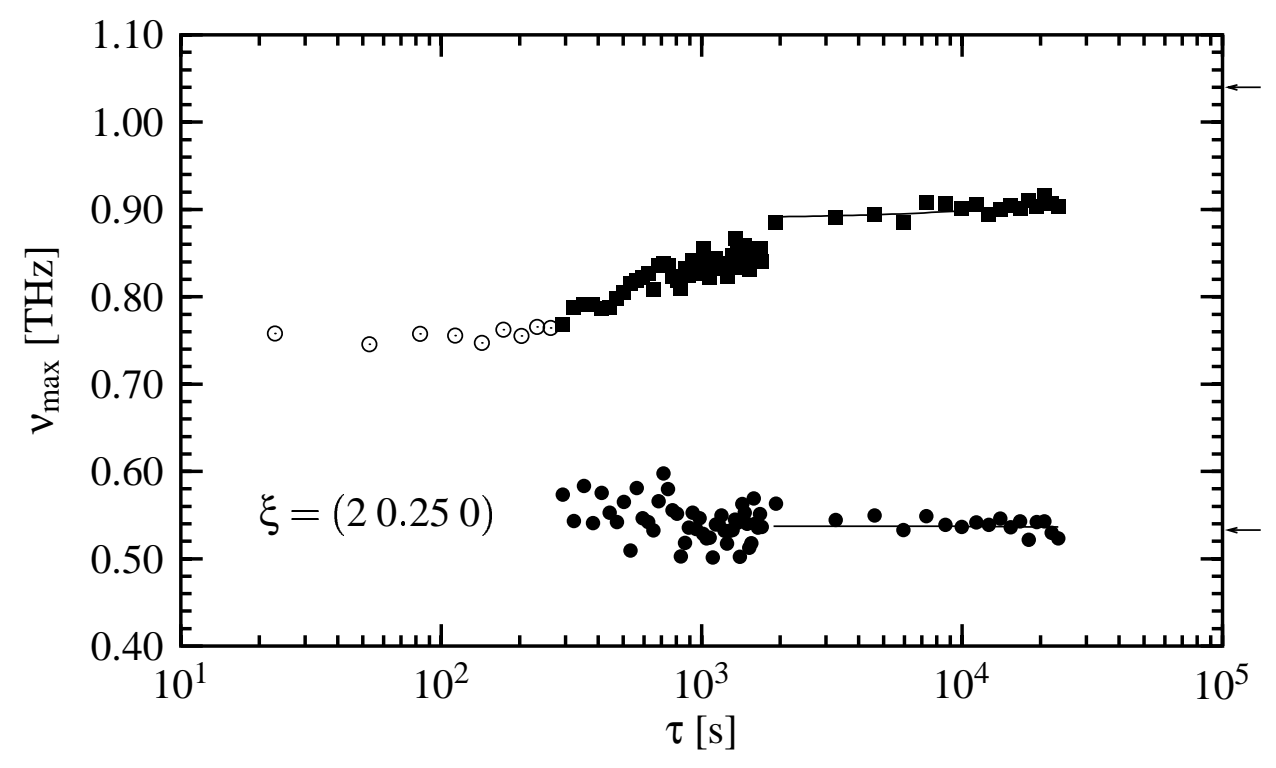

Abbildung 5.11: Die Frequenzaufspaltung über lange Zeiträume bei $107^{\circ} \mathrm{C}$ und $x_{0}=0.26$

\subsection{Entmischung in den späten Stadien}

In den späten Stadien der Entmischung muß die Frequenzaufspaltung weiter zunehmen wie der Vergleich mit dem quasistabilen Zustand, vgl. Abbildung 5.4 und 5.8, zeigt. Hierfür wurden an der $26 \%$-Probe bei $\xi=(20.250)$ neben stroboskopischen Experimenten zusätzlich sequentielle Experimente über längere Zeiträume durchgeführt. Es findet sich ebenso wie in den $41 \%$ - und $23 \%$-Proben wieder eine zunächst schnelle $\left(\tau<10^{3} \mathrm{~s}\right)$ und dann eine langsame, kontinuierliche Entmischung $\left(\tau>10^{3} \mathrm{~s}\right)$. Die Kinetik der frühen Entmischungsstadien darf aber aufgrund der start unterschiedlichen Abkühlraten, vgl. Tabelle 5.1, nicht mit der Kinetik der $23 \%$-Probe verglichen werden. Die Frequenz der silberreichen Phase liegt nach einigen $10^{3} \mathrm{~s}$ im Bereich des quasistabilen Zustandes, der wieder mit Pfeilen am rechten Rand der Auftragung gekennzeichnet ist. Bei einem Volumenanteil von $10 \%$ sind die silberreichen Phasen so groß, daß die Phononen die lokale Konzentration der silberreichen Phase wiedergeben. Die Frequenzänderung der natriumreichen Phase liegt auch nach mehr als $2 \cdot 10^{4} \mathrm{~s}$ erst bei $50 \%$ der Änderung, die nötig ist, um den quasistabilen Zustand zu erreichen. In diesem Zeitbereich ändert sich die Frequenz mit $0.08 \mathrm{THz} / \mathrm{d}$. Dieses Experiment schließt die zeitliche Lücke zwischen die frühen Stadien und dem quasistabilen Zustand. Die Entmischung verlangsamt zusehends, weil die Triebkraft der Entmischung, der Gradient des chemischen Potentials, abnimmt, und die Probe gelangt nur sehr langsam ins Gleichgewicht. 


\section{Zusammenfassung}

Im Einphasengebiet des Phasendiagramms liegen für die untersuchten Zusammensetzungen sowohl strukturell als auch chemisch wohl definierte homogene Phasen vor. Dieser homogene Zustand - mit den experimentellen Methoden dieser Arbeit untersucht — bleibt durch schnelles Abschrecken ins Zweiphasengebiet über einen Zeitraum von ca. $\tau=40$ s zunächst erhalten, und die Entmischung erfolgt aus dieser initialen homogenen Phase heraus. Nach dem Abschrecken in den instabilen Bereich des Phasendiagramme entmischt $\mathrm{Ag}_{x} \mathrm{Na} a_{1-x} \mathrm{Cl}$ innerhalb weniger hundert Sekunden in zwei Phasen mit den Zusammensetzungen $x_{1}^{\prime}$ und $x_{2}^{\prime}$ der kohärenten Spinodale. Diese schnelle chemische Entmischung erfolgt nach dem spinodalen Entmischungsmechanismus, bei dem sich die lokale Konzentration ausgehend von der mittleren Zusammensetzung kontinuierlich ändert. In Abbildung 6.1 ist die zeitliche Entwicklung der Konzentration für eine Probe mit einer mittleren Konzentration von $41 \%$ gezeigt.

An die schnelle chemische Entmischung schließt sich eine langsame Entmischung an, die mit der Relaxation des Gitters einhergeht. In den späten Stadien der Entmischung, im quasistabilen Zustand, treten Konzentrationsverteilungen in einem Intervall

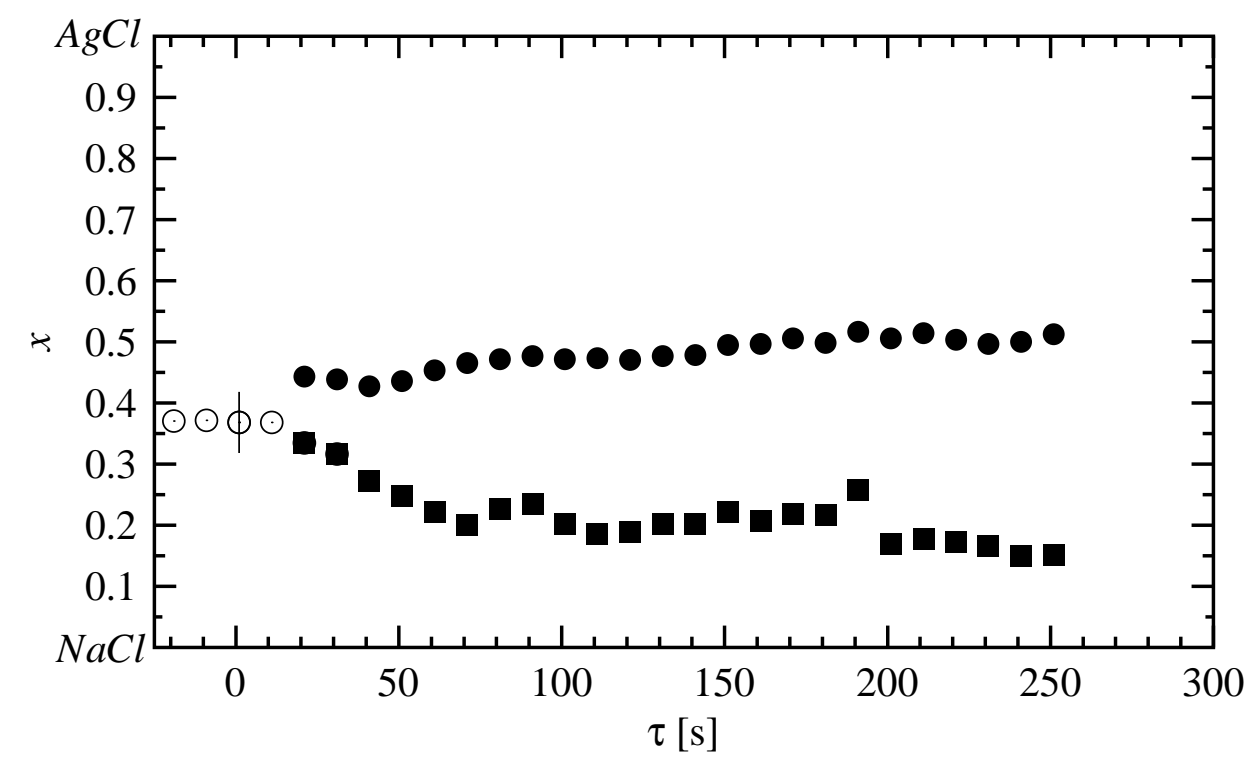

Abbildung 6.1: Die Zusammensetzung der dominierenden silber- und natriumreichen Phase im Verlauf der Entmischung bei $x_{0}=0.41$ 


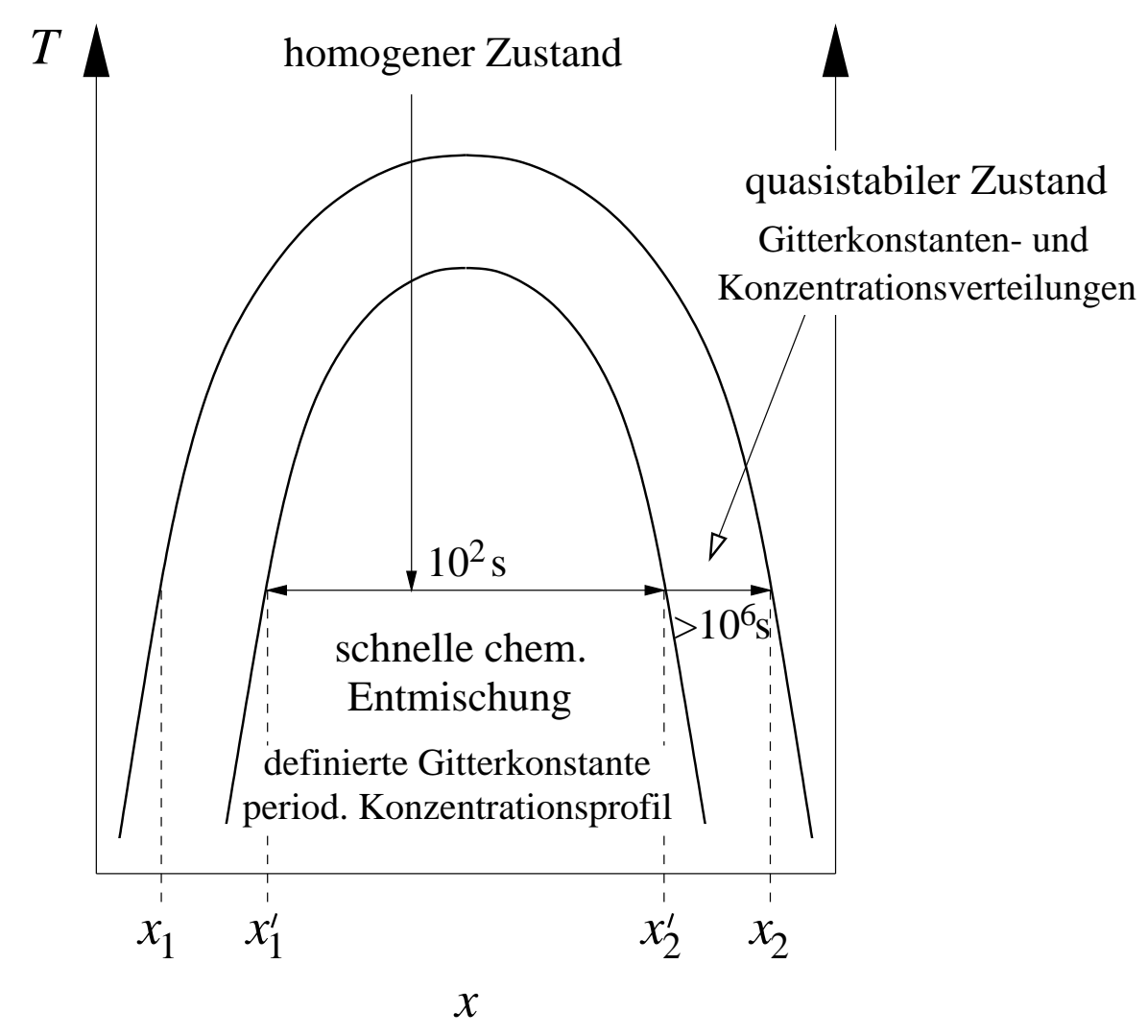

Abbildung 6.2: Übersicht zur Kinetik der Entmischung in $\mathrm{Ag}_{x} \mathrm{Na} a_{1-x} \mathrm{Cl}$

$x_{1} \ldots x_{1}^{\prime}$ bzw. $x_{2}^{\prime} \ldots x_{2}$ mit Gitterkonstantenverteilungen $a\left(x_{1} \ldots x_{0}\right)$ bzw. $a\left(x_{0} \ldots x_{2}\right)$ auf. Die Entmischungskinetik ist nochmals in Abbildung 6.2 zur Übersicht zusammengefaßt.

Neben der Aufklärung des Entmischungsmechanismus im instabilen Bereich des Phasendiagramms stehen die Entwicklung des portablen Systems für die stroboskopischen Experimente und die Ergebnisse zur Gitterdynamik. An Silber-Natriumchlorid und dem analogen Silber-Natriumbromid wurden erstmals zeitaufgelöste inelastische Neutronenstreuexperimente durchgeführt. Die stroboskopische Meßmethode ist dabei grundsätzlich auf beliebige Experimente anwendbar, so lange die im Abschnitt 3.4 gestellten Forderungen erfüllt werden. Des Weitern sind andere externe Parameter wie Druck, elektrisches Feld, u.s.w. möglich. Für die Gitterdynamik ergeben sich neue experimentelle Einsichten in die Abhängigkeit von Gitterschwingungen von der Konzentration bzw. dem Volumen einer Ausscheidung und der umgebenden Matrix. Es ist eine auf dieser Arbeit aufbauende theoretische Fortführung wünschenswert, in der die Gitterdynamik von kleinen kohärenten Ausscheidungen in einer Matrix untersucht wird. Dafür bietet es sich an, Experimente in Systemen mit $x_{0} \rightarrow x_{1}^{\prime}$ bzw. $x_{0} \rightarrow x_{2}^{\prime}$ wie SilberNatriumbromidsystem durchzuführen, deren Kinetik im quasistabilen Zustand schnel- 
ler ist.

Zur Zeit erfolgen auch analoge Untersuchungen an Silber-Natriumbromid, in denen sich der Erfolg der Experimentstrategie wiederholt. Es wäre aber wünschenswert, die Strategie zudem auf andere, metallische Systeme zu übertragen, um so die Methode zu etablieren und einem größeren Kreis bekannt zu machen. 


\section{Literaturverzeichnis}

[A.Bouvet und A.Filhol 1995] A.Bouvet ; A.FILHOL: User's manual of PkFit. In: ILL report $95 B O 21 T$ (1995) 44,55

[A.Bouvet u. a. 1997] A.Bouvet ; A.FILhol ; J.KuldA: PkFit - a tool to fit the data from neutron three-axis spectrometers. In: Nucl. Instr. and Meth. A 390 (1997), Nr. 3, S. 359-365 44, 55

[Agarwal und Hermann 1973] Agarwal, S. C. ; Hermann, H.: Phase decomposition in aluminum alloys quenched from the liquid state. In: HENISCH, H. K. (Hrsg.) ; Roy, R. (Hrsg.): Phase Transitions and their Applications in Material Science. N.Y. : Pergamon Press, 1973, S. 207-222 12

[Aslam u. a. 1976] Aslam, J. ; Beg, M. M. ; Butt, N. M. ; Khan, Q. H.: Phonons in the Mixed Crystal $K_{0.5} R b_{0.5} I$. In: Phys. Stat. Sol. B 77 (1976), S. 693-700 31

[Bartel und Rundmann 1975] Bartel, T. L. ; Rundmann, K. B.: Decomposition in Al-Zn Alloys: Part I. Isothermal Decomposition in an Al-22 At. Pct Zn-0.1 At. Pct Mg Alloy. In: Metall Trans 6 A (1975), S. 1887-1893 12

[Bilz und Kress 1979] BILz, H. ; Kress, W.: Phonon Dispersion Relation in Insulators. Berlin, Heidelberg, New York : Springer Verlag, 1979 (Springer series in solid-state sciences 10) 32

[Binder 1986] Binder, K.: Decay of metastabile and unstable states: Mechanisim, concepts and open problems. In: Pysica 140a (1986), S. 35-43 12

[Binder u. a. 1978] Binder, K. ; Billotet, C. ; Mirold, P.: On the Theory of Spinodal Decomposition in Solid and Liquid Mixtures. In: Z. Physik B 30 (1978), S. 183-195 13

[Binder und Stauffer 1974] Binder, K. ; Stauffer, D.: Theory for the Slowing Down of the Relaxation and Spinodal Decoomposition of binary Mixtures. In: Phys. Rev. Lett. 33 (1974), Nr. 17, S. 1006-1009 13

[Brückel u. a. 2000] BRÜCKEL, Thomas (Hrsg.) ; HEgER, Gernot (Hrsg.) ; RICHTER, Dietrich (Hrsg.): Materie und Material. Bd. 5: Neutron Scattering, Lectures of the Laboratory Course. Jülich : Zentralbibliothek des Forschungszentrum Jülich, 200018 
[Brenner u. a. 1984] Brenner, S. S. ; CAmus, P. P. ; Miller, M. K. ; Soffa, W. A.: Phase Separation and Coarsening in Fe-Cr-Co. In: Acta metall. 32 (1984), Nr. 8, S. $1217-122712$

[Brunner-Popela und Glatter 1997] Brunner-Popela, J. ; Glatter, O.: SmallAngle Scattering of Interacting Particles. I. Basic Principles of a Global Evaluation Technique. In: J. Appl. Cryst. 30 (1997), S. 431-442 27

[Cahn 1961] Cahn, J. W.: On Spinodal Decomposition. In: Acta Metall. 9 (1961), S. 79510

[Cahn 1962] CAHN, J. W.: Coherent fluctuations and nucleation in isotropic solids. In: Acta Metall. 10 (1962), S. 907-913 10

[Cahn 1966] CAHN, J. W.: The later stages of spinodal decomposition and the beginning of particle coarsening. In: Acta Metall. 14 (1966), S. 1685-1692 10

[Cahn und Hilliard 1958] Cahn, J. W. ; Hilliard, J. E.: Free Energy of a Nonuniform System. I. Interfacial Free Energie. In: J. Chem. Phys. 28 (1958), Nr. 2, S. 258-267 10

[Cahn und Hilliard 1959a] CAhn, J. W. ; Hilliard, J. E.: Free Energy of a Nonuniform System. II. Thermodynamik Basis. In: J. Chem. Phys. 30 (1959), Nr. 5, S. $1121-112410$

[Cahn und Hilliard 1959b] Cahn, J. W. ; Hilliard, J. E.: Free Energy of a Nonuniform System. III. Nucleation in a Two-Component Incompressible Fluid. In: $J$. Chem. Phys. 31 (1959), Nr. 3, S. 688-699 10

[Caspary u. a. 2001] Caspary, D. ; Eckold, G. ; Güthoff, F. ; PyckhoutHIntzen, W.: Kinetics of Decomposition in Ionic Solids II: Neutron Scattering Study of the System AgCl-NaCl. In: J. Phys: Condens. Matter 13 (2001), S. 11521-11530 27, 30, 31, 50, 66, 75, 81

[Caspary 1998] CASPARY, Dirk: Untersuchungen zur spinodalen Entmischung im System AgCl-NaCl mit zeitaufgelöster Neutronenkleinwinkelstreuung, Universität Göttingen, Diplomarbeit, 1998 25, 27, 30, 50, 66

[Cook 1969] CoOK, H. E.: The kinetics of clustering and short-range order in stable solid solutions. In: J. Phys. Chem. Solids 30 (1969), S. 2427-2437 10

[Cook 1970] CooK, H. E.: Brownian Motion in Spinodal Decomposition. In: Acta Metall. 18 (1970), S. 297-306 10 
[Cooper und Nathans 1967] Cooper, M. J. ; NAthans, R.: The Resolution Function in Neutron Diffractomeriy. I. The Resolution Function of a Neutron Diffractometer and its Application to Phonon Measurements. In: Acta Cryst. 23 (1967), Nr. 3, S. 357-367 44

[Cowley 1967] Cowley, R. A.: Zero sound, first sound and second sound of solids. In: Proc. Phys. Soc. 90 (1967), S. 1127-1147 57, 70

[Cullity 1978] Cullity, Bernard D.: Elements of X-Ray Diffraction. 2. AddisonWesley, 1978 (Addison-Wesley Series in Metallurgy ans Materials) 65

[Doi u. a. 1984] DoI, Mindoru ; MiYAZAKI, Toru ; WAKATsuKi, Teruyuki: The Effect of Elastic Interaction Energy on the Morphology of $\gamma^{\prime}$ Precepirations in Nickelbase Alloys. In: Mater. Sci. Eng. 67 (1984), S. 247-253 9

[Dorner 1982] DoRner, B.: Coherent Inelastic Neutron Scattering. Berlin, Heidelberg, New York : Springer, 1982 (Springer Tracts in Modern Physics 93) 18, 22

[Dove 1993] Dove, Martin T.: Introduction to Lattice Dynamics. Camebridge : Cambridge University Press, 1993 (Cambridge Topics in Mineral Physics and Chemistry) 16

[Eckerlebe u. a. 1986] Kap. SANS-Investigations of Early Stage Precipitation Kinetics in $\mathrm{Cu}-2.9 \% \mathrm{Ti}$ In: Eckerlebe, H. ; Kampmann, R. ; Wagner, R.: Atomic Transport and Defects by Neutron Scattering. Berlin : Springer, 1986 (Springer proceedings in physics 10), S. 66-72 12

[Eckold 1990] EcKOLD, G.: Time-resolved tripple-axis spectroscopy - A new method for real-time neutron scattering. In: Nucl. Instr. and Meth. A289 (1990), S. 22123049

[Eckold 1991] ECKOLD, G. Nicht-Gleichgewichtszustände bei Phasenmwandlungen. Jül. Report 2675. 1991 26, 49

[Eckold 1992] Eckold, G.: UNISOFT - A Program Package for Lattice Dynamical Calculations. FZ Jülich, 1992. - ISSN 0366-0885 16, 33

[Eckold 1994] EcKOLD, G.: Dynamik von Kristallen I,Gitterdynamik. 1994. - Vorlesungsscript Wintersemester 1994/95 16

[Eckold und Mitlacher 1982] EcKold, G. ; Mitlacher, Horst: UNIDASBenutzerhandbuch, 198244 
[Eckold 2001] ECKOLD, Götz: Kinetics of decomposition in ionic solids: neutron scattering study of the System AgBr-NaBr. In: J. Phys.: Condens. Matter 13 (2001), S. 217-240 26

[Elisbihani 2002] ElisBIHANI, Khalid: Hochauflösende $\gamma$-Diffraktometrie zut Untersuchung der ferroelektrischen Lock-in Phasenumwandlung in $\mathrm{Rb}_{2} \mathrm{ZnCl}_{4}$, Universität Göttingen, Dissertation, 200241

[Elter und Eckold 2000] ELTER, P. ; ECKOLD, G.: Visualising lattice vibrations: new features of the UNISOFT-programm. In: Physica B 276-278 (2000), S. 268-269 16,33

[Every und McCurdy 1992] Every, A. G. ; McCurdy, A. K. ; Nelson, D. F. (Hrsg.): Landold-Börnstein Neue Serie. Bd. III/29a. Springer Verlag, 199234

[Falbe und Gefitz 1991] Kap. N In: FALBE, Jürgen (Hrsg.) ; GefiTZ, Manfred (Hrsg.): Römpp Chemie Lexikon. Georg Thieme Verlag, 1991, S. 2922-2923 25

[Falbe und Gefitz 1992] Kap. S In: FALBE, Jürgen (Hrsg.) ; GeFITZ, Manfred (Hrsg.): Römpp Chemie Lexikon. Georg Thieme Verlag, 1992, S. 4156-4157 25

[Feigin und Svergun 1987] Feigin, L. A. ; Svergun, D. I.: Structure Analysis by Small Angle X-Ray and Neutron Scattering. New York : Plenum Press, 198727

[Fischer u. a. 1972] Fischer, K. ; Bilz, H. ; HAberkorn, R. ; Weber, W.: Covalency and Doformability of $\mathrm{Ag}^{+}$-Ions in the Lattice Dynamics of Silver Halides. In: Phys. Stat. Solidi (B) 54 (1972), Nr. 1, S. 285-299 33

[Furukawa 1984] FURUKAWA, Hiroshi: Dynamic-scaling theory for phase-seperating unmixing mixtures: Growth rates of droplets and scaling properties of autocorrelation functions. In: Physica 123A (1984), S. 497-515 28

[Gerold 1961] Gerold, V.: Die Zonenbildung in Aluminium-Zink-Legierungen. In: Phys. Stat. Solidi. 1 (1961), Nr. 1, S. 37-49 29

[Gerold 1978] Gerold, V.: Small-Angle Scattering Applications to Materials Sience. In: J. Appl. Cryst. 11 (1978), S. 376-404 27

[Gerold 1967] GEROLD, Volkmar: Application of Small-Angle X-Ray Scattering to Problems in Physical Metallurgy and Metall Physics. In: Small-Angle X-Ray Scattering, 196727

[Glatter und Kratky 1982] Glatter, O. (Hrsg.) ; Kratky, O. (Hrsg.): Small Angle X-Ray Scattering. London : Academic Press, 198227 
[Gmelin 1971] GMELIN: Gmelins Handbuch der Anorganischen Chemie. Weinheim/Bergstraße : Verlag Chemie, 197169

[Gmelin 1972] In: GMELIN (Hrsg.): Gmelins Handbuch der Anorganischen Chemie. Weinheim/Bergstraße : Verlag Chemie, 1972, S. 317-426. - Syst.-Nr. 61, Ag, Tl. B 125

[Goyal und Asthana 1991a] Goyal, R. P. ; Asthana, Sunil: Phonon Dispersion in Mixed Alkali Halide Crystal $\mathrm{KCl}_{0.5} \mathrm{Br}_{0.5}$. In: Infrared Phys. 31 (1991), Nr. 5, S. 413-414 31

[Goyal und Asthana 1991b] Goyal, R. P. ; Asthana, Sunil: Phonon Dispersion in Mixed Crystal $K_{0.5} R b_{0.5} C l$. In: Infrared Phys. 31 (1991), Nr. 5, S. 195-198 31

[Grünewald und Scharnberg 1978] GRÜnEwALD, G. ; SCHARnBERG, K.: Lattice Dynamics of Substitutional Alloys of Simple Metals Including Force Constant Disorder. In: BALKANSKI, M. (Hrsg.): Lattice dynamics - International Conference Paris, September 1977. Paris : Flammarion Sciences, 1978, S. 443-446 31

[Guinier und Fournet 1955] Guinier, A. ; Fournet, G.: Small-Angle Scattering of X-Rays. New York : John Wiley \& Sons, 1955. - Translation by C. B. Walker 27

[Hawick 1991] HAWICK, Kenneth A.: Domain Growth in Alloys, Universität Edinburgh, Dissertation, 199112

[Hendricks u. a. 1964] HENDRICKS, R. W. ; BARO, R. ; NEWKIRK, J. B.: Precipitation of $\mathrm{AgCl}$ in $\mathrm{NaCl}(\mathrm{AgCl})$ Solid Solutions. In: Transactions of the Metallurgical Society of AIME 230 (1964), S. 930-931 26

[Hollemann und Wiberg 1985] Hollemann, A. F. ; WiBerg, E.: Lehrbuch der Anorganischen Chemie. 91.-100. Berlin, New York : Walter de Gruyter, 1985. - S. $1015 \mathrm{ff} 25$

[Hurle 1993] HuRLE, Donald T. J.: Crysral pulling from the melt. Berlin : Springer, 199337

[Hutten und Grune 1986] Hutten, A. ; GRune, R.: Long Range order in homogenized AlNiCo. In: Script. metall. 20 (1986), Nr. 4, S. 551-554 12

[Jantzen und Herman 1978] Kap. Spinodal Decomposition In: JAnTZEN, C. ; HeRMAN, H.: Phase Diagrams and Materials Science and Technology. Bd. V. Academic Press and New York, San Francisco, London, 1978, S. 128-184 25

[Kittel 1996] KitTEL, Charles: Einführung in die Festkörperphysik. 11. München : R. Oldenbourg Verlag, 199630 
[Langer 1971] LANGER, J. S.: Theory Of Spinodal Decomposition in Alloys. In: J. Phys. 65 (1971), S. 53-86 13

[Langer u. a. 1975] Langer, J. S. ; BAR-On, M. ; Miller, H. D.: New Computational Method In The Theory Of Spinodal Decomposition. In: Phys. Rev. A 11 (1975), Nr. 4, S. 1417-1429 13

[Lewandowski und Rundmann 1975] Lewandowski, D. T. ; Rundmann, K. B.: Decomposition in Al-Zn Alloys: Part II. Decomposition During Continous Cooling. In: Metall Trans 6 A (1975), S. 1895-1900 12

[Lifshitz und Slyozov 1961] LifShitz, I. M. ; Slyozov, V. V.: The kinetics of precipitation from supersatured solid solutions. In: J.Phys.Chem. Solids 19 (1961), Nr. 3510,28

[Lovesey 1984] LOVESEY, Stephen W.: Theory of Neutron Scattering from Condenced Matter. Bd. 1. 1. Oxford : Clarendon Press, 198418

[Madeira 1989] MAdEIRA, H. T. Y.: Untersuchung der Ausscheidungskinetik der Polymermischung aus deuteriertem Polystyrol (d-PS) und Polyvinylmethylether ( PVME) mittels Neutronen-Kleinwinkel-Streuung, RWTH Aachen, Diss., 198912

[Maier 2000] MAIER, J.: Festkörper - Fehler und Funktion. Teubner, 2000 (Teubner Studienbücher Chemie) 14

[Pamplin 1980] PAmplin, Brain R. (Hrsg.): International Series on the Science of the Solid State. Bd. 16: Crystal Growth. 2. Oxford : Pergamon Press, 198037

[Perry u. a. 1978] Perry, C. H. ; Jahn, I. R. ; WAgner, V. ; BAuhofer, W. ; SoKoLOFF, J. B.: Phonon Dispersion in $\mathrm{NH}_{4} \mathrm{Cl}_{1-x} \mathrm{Br}_{x}$ Mixed Crystals. In: BALKANSKI, M. (Hrsg.): Lattice dynamics — International Conference Paris, September 1977. Paris : Flammarion Sciences, 1978, S. 419-422 31

[Putnis 1993] Putnis, Andrew: Introduction to Mineral Siences. Cambridge University Press, 1993 8, 10

[Scherm 1972] SCHERM, R.: Fundamentals of Neutron Scattering by Condensed Matter. In: Ann. Phys. 7 (1972), Nr. 5, S. 349-370 18, 21

[Schimschal-Thölke u. a. 1995] Schimschal-ThöLKe, S. ; SchmalzRIEd, H. ; M.MARTIN: Stability of Diffusion Profiles in quasi-binary Solid Solutions (Ag, Na)Cl. In: Ber. Bunsenges. Phys. Chem. 99 (1995), Nr. 1, S. 7-13 25 
[Schmunk und Winder 1970] Schmunk, R. E. ; WindeR, D. R.: Lattice Dynamics of Sodium Chloride at Room Temperature. In: J. Phys. Chem. Solids 31 (1970), S. 131-141 33

[Sears 1992] SEARS, Valery F.: Neutron scattering lengths and cross sections. In: Neutron News 3 (1992), Nr. 3, S. 26-37 19

[Shapiro und Chasser 1972] ShapIRo, S. M. ; ChASSER, N. J.: Characteristics of pyrolytic graphite as an analyser and higher order filter in neutron scattering experiments. In: Nucl. Instr. and Meth. 101 (1972), S. 183-186 105

[Sinistri u. a. 1971] SinistRI, Ceasare ; RICCARDI, Riccardo ; MARGHERITIS, Chiara ; Tittarelli, Paolo: Thermodynamic Properties of Solid Systems AgCl+NaCl and $\mathrm{AgBr}+\mathrm{NaBr}$ from Miscibility Gap Measurements. In: Z. Naturforsch. 27a (1971), S. 149-154 25, 26

[Squires 1978] SQUIRES, G. L.: Introduction to the Theory of Thermal Neutron Scattering. Cambridge, London, New York, Melbourne : Cambridge University Press, 197818

[Stauffer und Aharony 1995] STAufFer, Dietrich ; AhARONy, Amnon: Perkolationstheorie, eine Einführung. Weinheim : VCH, 199512

[Stokes und Li 1962] StokeS, R. J. ; LI, C. H.: The Sodium Chloride-Silver Chloride Alloy System. In: Acta metall. 10 (1962), S. 535-542 25, 38, 40

[Sturm 1996] STURM, K.: Wechselwirkung von thermischen Neutronen und Röntgenstrahlen mit Atomkernen und Elektronen. In: IFF Ferienschule Streumethoden zur Untersuchung kondensierter Materie. FZ Jülich, 199618

[Trzeciok 1976] TRZECIOK, D.: Röntgenmessungen am System NaBr-AgBr, Universität Göttingen, Dissertation, 197626

[Ubbelohe 1965] Ubbelohe, A. R.: Melting and Crystal Structure. Oxford : Clarendon Press, 196559

[Vijayaraghaven u. a. 1970] VijayaraghaVen, P. R. ; Nicklow, R. M. ; Smith, H. G. ; Wilkinson, M. K.: Lattice Dynamics of Silver Chloride. In: Phys. Rev. B 1 (1970), Nr. 12, S. 4819-4826 33

[Windgasse u. a. 1997] Windgasse, J. ; ECKOld, G. ; GÜThOFF, F.: Decomposition in AgBr-NaBr single crystals. In: Physica B 234-236 (1997), S. 153-154 26 
[Windgasse 1994] WINDGASSE, Jochen: Czochralski-Züchtung von $A g_{x} N a_{(1-x)} B r$ Mischkristallen. Kernforschungsanlage Jülich. 1994. - Studienarbeit am Institut für Kristallographie der RWTH Aachen 26, 37

[Windsor 1988] WindSOR, Colin G.: An Introduction to Small-Angle Neutron Scattering. In: J. Appl. Cryst. 21 (1988), S. 582-588 27

[Zhu u. a. 1986] Zhu, F. ; HaAsen, P. ; Wagner, R.: An Atom Probe Study of the Decomposition of Fe-Cr-Co Permanent Magnet Alloys. In: Acta Metall. 34 (1986), Nr. 3, S. 457-463 12 


\section{Abbildungsverzeichnis}

1.1 Zusammenhang zwischen Phasendiagramm und freie Enthalpie . . . . 7

1.2 Hetero- und Homophasenfluktuationen . . . . . . . . . . . . . . . 9

1.3 Konzentrationsverteilungen aus einer zweidimensionalen Computersimulation der spinodalen Entmischung . . . . . . . . . . . . . 12

1.4 Wechselwirkungen zwischen Atomen im Schalenmodell . . . . . . . . 15

1.5 Zur Ausbreitung von longitudinalen und trasversalen Wellen . . . . . . 16

1.6 Zur Streugeometrie zweier Streuzentren und das Streudreieck im elastischen Fall . . . . . . . . . . . . . . . . . . . . . . . 18

1.7 Anschauliche Darstellung des doppelt differnziellen Streuquerschnitts . 22

1.8 Das Streudreieck im inelastischen Fall . . . . . . . . . . . . . . . . 23

2.1 Die Mischungslücke von $\mathrm{Ag}_{x} \mathrm{Na}_{1-x} \mathrm{Cl} \ldots \ldots \ldots \ldots \ldots$

2.2 SAns -Diffraktogramme und $Q_{\max }$ vs. $t \ldots \ldots$. . . . . . . . 28

2.3 SANS Integrale Intensität . . . . . . . . . . . . . . . . . . . . . 29

2.4 Die kohärente Spinodale von $\mathrm{Ag}_{x} \mathrm{Na}_{1-x} \mathrm{Cl} \ldots \ldots \ldots$. . . . . . . . . . . . 31

2.5 Die TA und LA Dispersion von reinem $\mathrm{AgCl}$ und $\mathrm{NaCl}$. . . . . . . . . 32

3.1 Kristallzucht nach dem Czochralski-Verfahren . . . . . . . . . . . . . 38

3.2 Das Schmelzgleichgewicht von $\mathrm{Ag}_{x} \mathrm{Na}_{1-x} \mathrm{Cl} \ldots \ldots$. . . . . . . . . . . . 39

3.3 Fotos von den Probenkristallen und deren Mosaizitäten . . . . . . . . . 42

3.4 Schematischer Aufbauf eines Neutronendreiachsspektrometers . . . . . 44

3.5 Der Probenaufbau am IN-12 . . . . . . . . . . . . . . . . 45

3.6 Zur Auflösung eines Neutronendreiachsspektrometers . . . . . . . . . . 46

3.7 Neutronenöfen . . . . . . . . . . . . . . . . . . . . . . . 47

3.8 Der Probenaufbau am UnIDAs . . . . . . . . . . . . . . . . . . . 48

3.9 Spektren zur Charakterisierung der Homogenisierungszeiten . . . . . . 51

3.10 Pulsdiagramme und Blockbild der verwendeten Elektronik . . . . . . . 52

4.1 Phononenspektrum bei $400{ }^{\circ} \mathrm{C}$ und $x_{0}=0.41 \ldots \ldots$. . . . . . . 56

4.2 Die Dispersion in der homogenen Phase bei $x_{0}=0.26$. . . . . . . . . 57

4.3 Die Abhängigkeit von $v$ des TA Phonons von $x \ldots \ldots$. . . . . . . 60

4.4 Zur Berechnung der Abhängigkeit von $v$ des TA Phonons von $x$. . . . . 63

4.5 Braggreflexe nach langen Auslagerungszeiten bei $x_{0}=0.41 \ldots$. . . . . 64

4.6 Braggreflexe nach langen Auslagerungszeiten bei $x_{0}=0.23 \ldots$. . . . . 65 
4.7 Spektren der TA Phononen nach langen Auslagerungszeiten . . . . . . 68

4.8 Die Dispersionskurven nach langen Auslagerungszeiten in $\left[\begin{array}{lll}1 & 0 & 0\end{array}\right]$. . . . 71

5.1 Die zeitabhängige Lage der Satelliten . . . . . . . . . . . . . . 77

5.2 Spektren für $x_{0}=0.41$ während des Abkühlens . . . . . . . . . . 78

5.3 Die zeitliche Entwicklung des Spektrums bei $x_{0}=0.41 \ldots$. . . . . . 79

5.4 Die Frequenzaufspaltung des TA Phonons bei $x_{0}=0.41 \ldots$. . . . . . 80

5.5 Die Halbwertsbreiten und die Geräteauflösung bei $x_{0}=0.41 \ldots$. . . . 82

5.6 Zur Entfaltung der Halbwertsbreite . . . . . . . . . . . . . . . 83

5.7 Die zeitliche Entwicklung des Spektrums für $x_{0}=0.23 \ldots \ldots$. . . . 85

5.8 Die Frequenzaufspaltung des TA Phonons bei $x_{0}=0.23 \ldots \ldots$. . . . 86

5.9 Die zeitabhängigen Halbwertsbreiten bei $x_{0}=0.23 \ldots \ldots$. . . . . 88

5.10 Die Abhängigkeit der mittleren Phononenfrequenz vom Volumenanteil . 89

5.11 Die Frequenzaufspaltung über lange Zeiträume bei $x_{0}=0.26 \ldots$. . . . 90

6.1 Die zeitabhängige Entwicklung der Konzentration während der Entmischung bei $x_{0}=0.41 \ldots \ldots \ldots \ldots$. . . . . . . . . . . . . . . . . .

6.2 Übersicht zur Kinetik der Entmischung in $\mathrm{Ag}_{x} \mathrm{Na}_{1-x} \mathrm{Cl} \ldots \ldots$. . . . . . . . 92

.3 Die erste Brillouin-Zone und für ein fcc-Gitter . . . . . . . . . . . . . . . . . . . . . . . . . . .

.4 Die Reflektivität von pyrolytischem Graphit . . . . . . . . . . . . . . 105

.5 Beispielmessung aus den diffusen Neutronenstreuexperimenten . . . . . 106

.6 Dispersion des TA Phonons in $\left[\begin{array}{lll}1 & 0 & 0\end{array}\right]$ nach langen Auslagerungszeiten

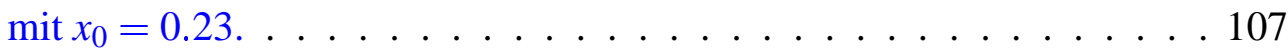

7 Die Dispersionskurven des TA Phonons in $\left[\begin{array}{lll}1 & 1 & 0\end{array}\right]$ nach langen Auslagerungszeiten mit $x_{0}=0.26 \ldots \ldots$. . . . . . . . . 107

.8 Die Abkühlkurve für $100{ }^{\circ} \mathrm{C}$ bei $x_{0}=0.41 \ldots \ldots$. . . . . . . . . . 108

.9 Die Abkühlkurve für $100{ }^{\circ} \mathrm{C}$ bei $x_{0}=0.23 \ldots \ldots$. . . . . . . 108

10 Die Abkühlkurve für $107^{\circ} \mathrm{C}$ bei $x_{0}=0.26 \ldots$. . . . . . . . . . . . 109

.11 Die Auflösung des Spektrometers bei den kinetischen Experimenten für $x_{0}=0.23 \ldots \ldots \ldots \ldots \ldots$ 


\section{Abbildungsanhang}

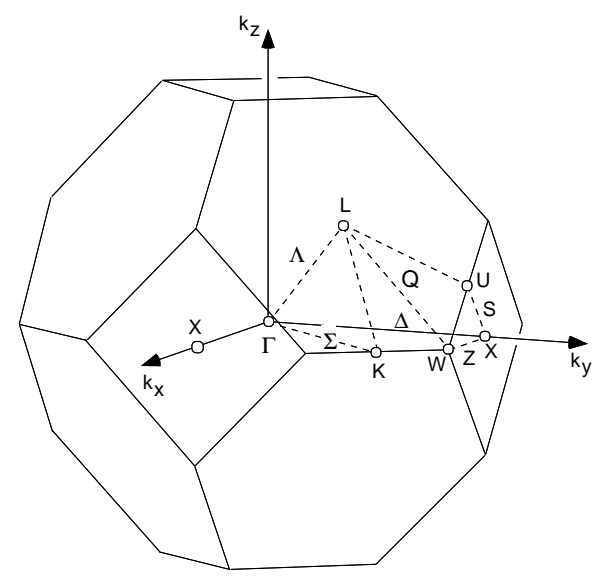

Abbildung .3: Die erste Brillouin-Zone und für ein kubisch flächenzentriertes Gitter

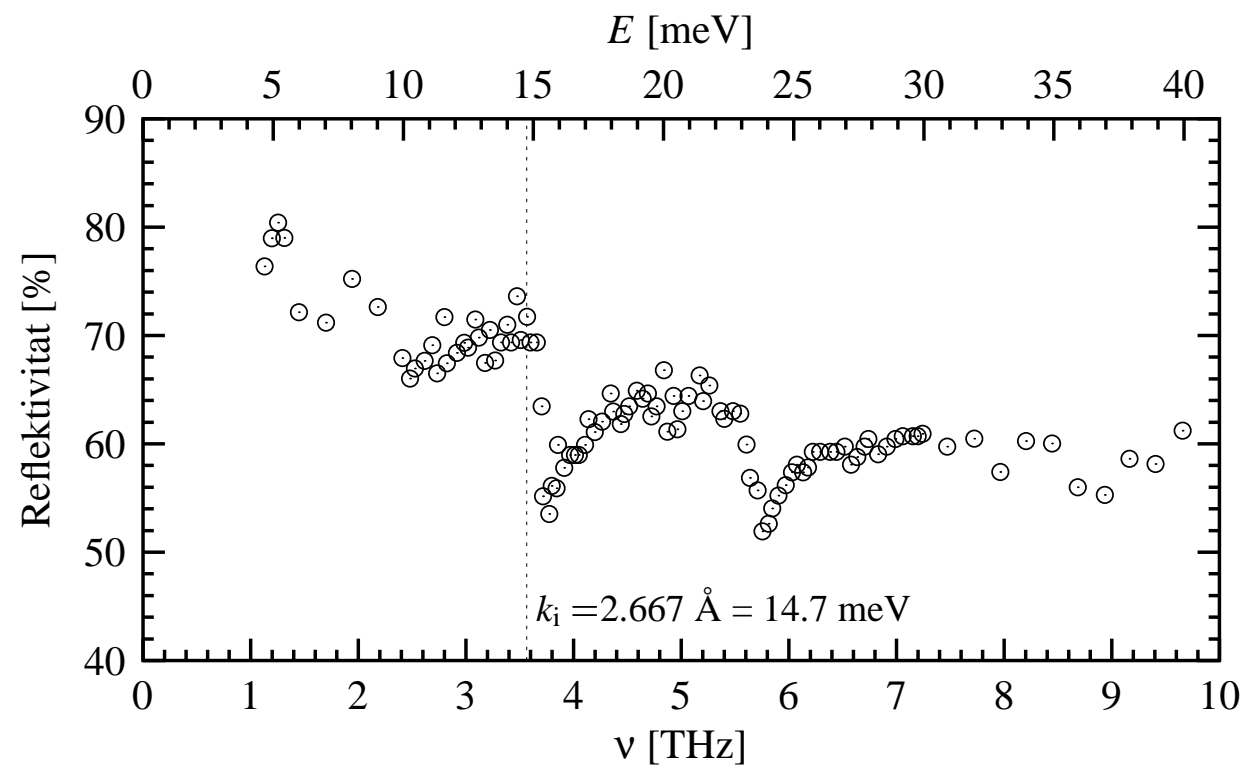

Abbildung .4: Die Reflektivität von pyrolytischem Graphit am (002)-Reflex nach [Shapiro und Chasser 1972] 


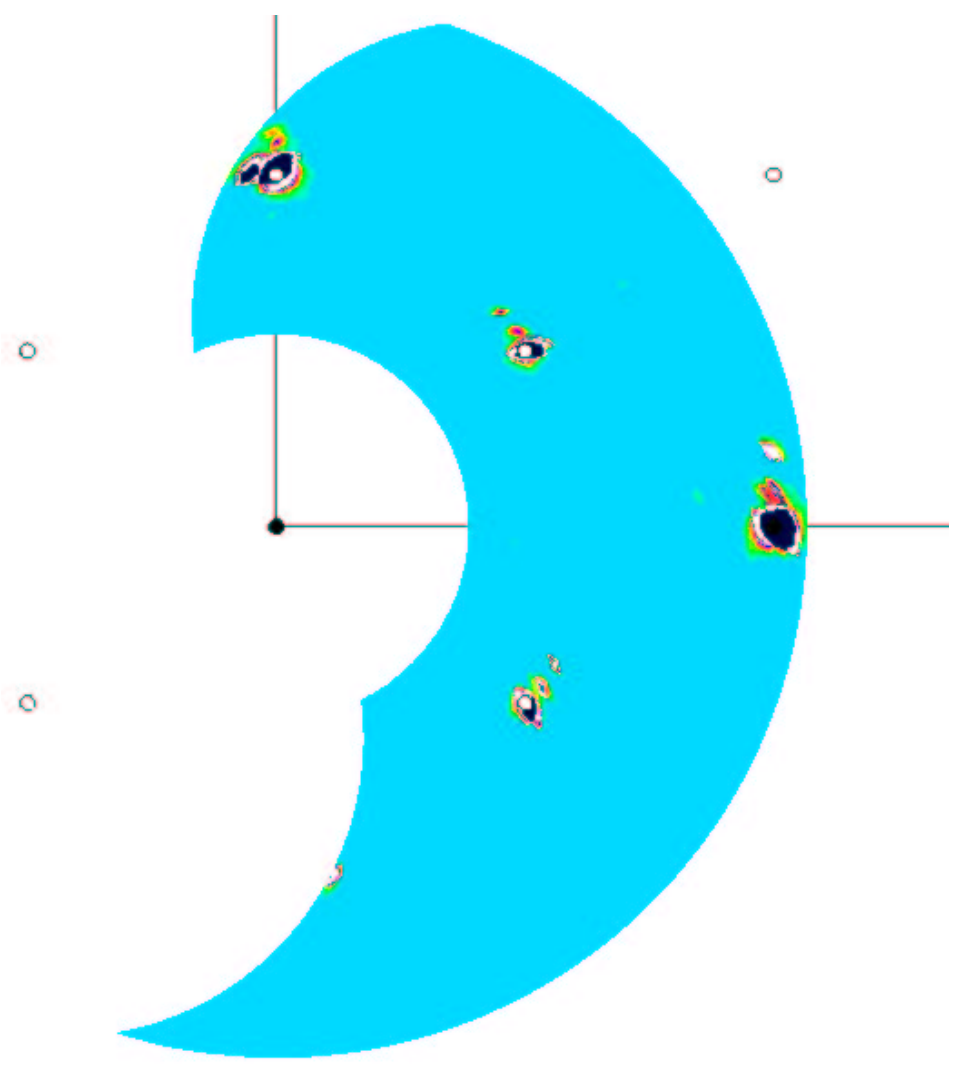

Abbildung .5: Die Abbildung zeigt die $\left[\begin{array}{lll}0 & 1 & \overline{1}\end{array}\right]$-Streuebene, wie sie auch in Abbildung 2.5 gezeigt ist. Es sind (2 000$)$ - und (0 2 2)-Braggreflex zu erkennen, die auf den eingezeichneten Achsen liegen. Ober- und unterhalb der $\left[\begin{array}{lll}1 & 0 & 0\end{array}\right]$-Achse liegen der $\left(\begin{array}{lll}1 & 1 & 1\end{array}\right)$ - und der $\left(\begin{array}{lll}1 & \overline{1} & \overline{1}\end{array}\right)$-Reflex. Die Intensitäten, die sich im mathematischen Drehsinn neben den Reflexen findet, sind auf die Mosaizität der Probe zurückzuführen. 


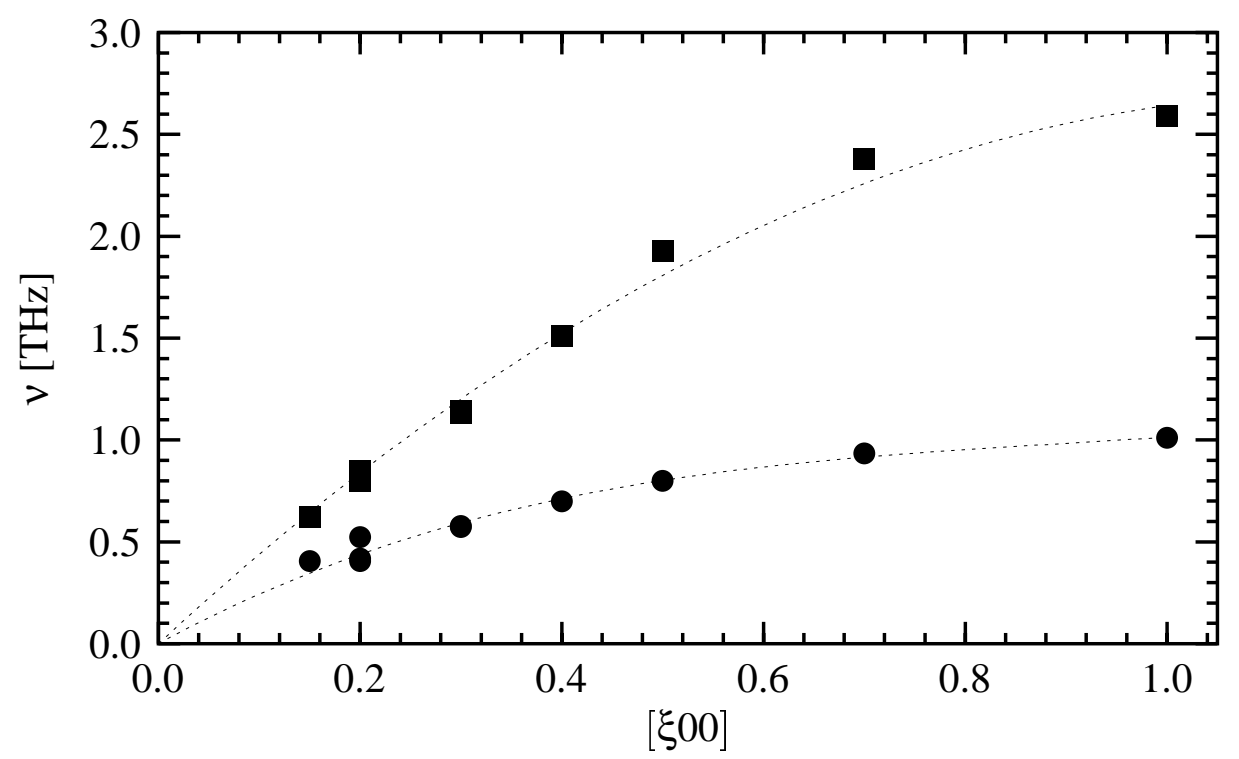

Abbildung .6: Dispersion des TA Phonons in $\left[\begin{array}{lll}1 & 0 & 0\end{array}\right]$ nach langen Auslagerungszeiten mit $x_{0}=$ 0.23 .

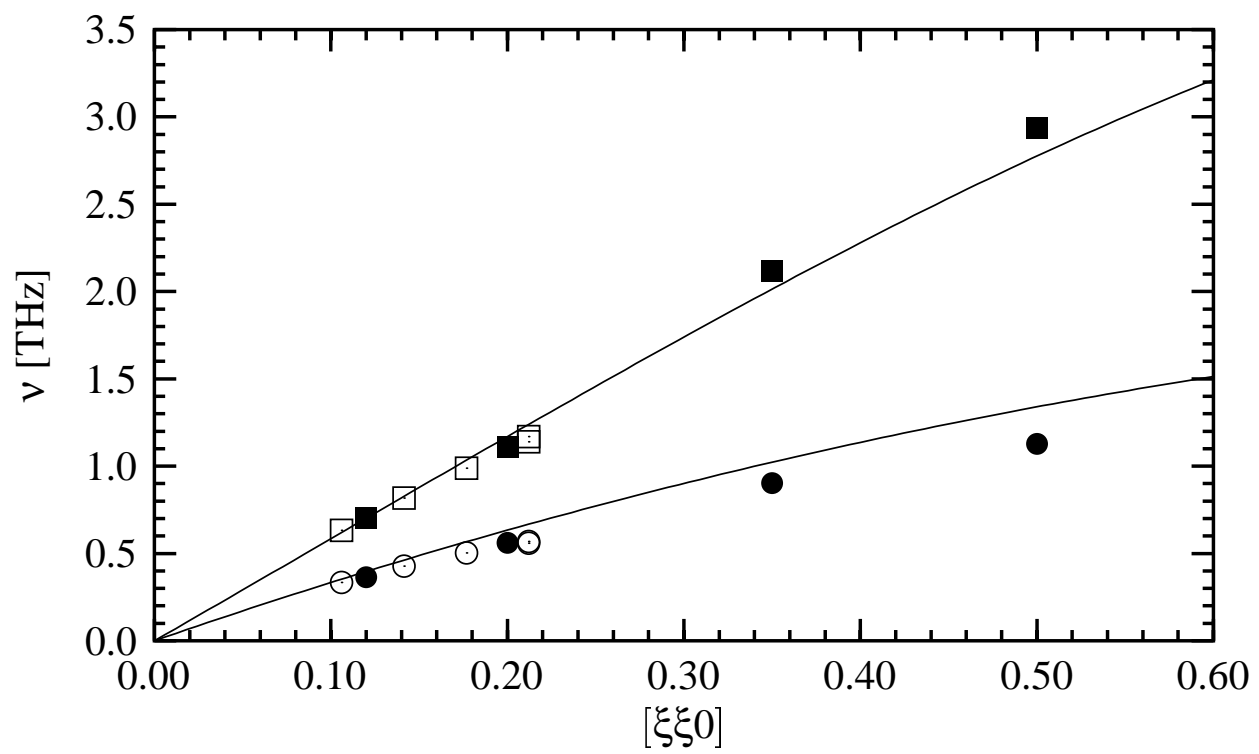

Abbildung .7: Die Dispersionskurven des transversal akustischen Phonons in $\left[\begin{array}{lll}1 & 1 & 0\end{array}\right]$ bei Raumtemperatur nach langen Auslagerungszeiten mit $x_{0}=0.26$ 


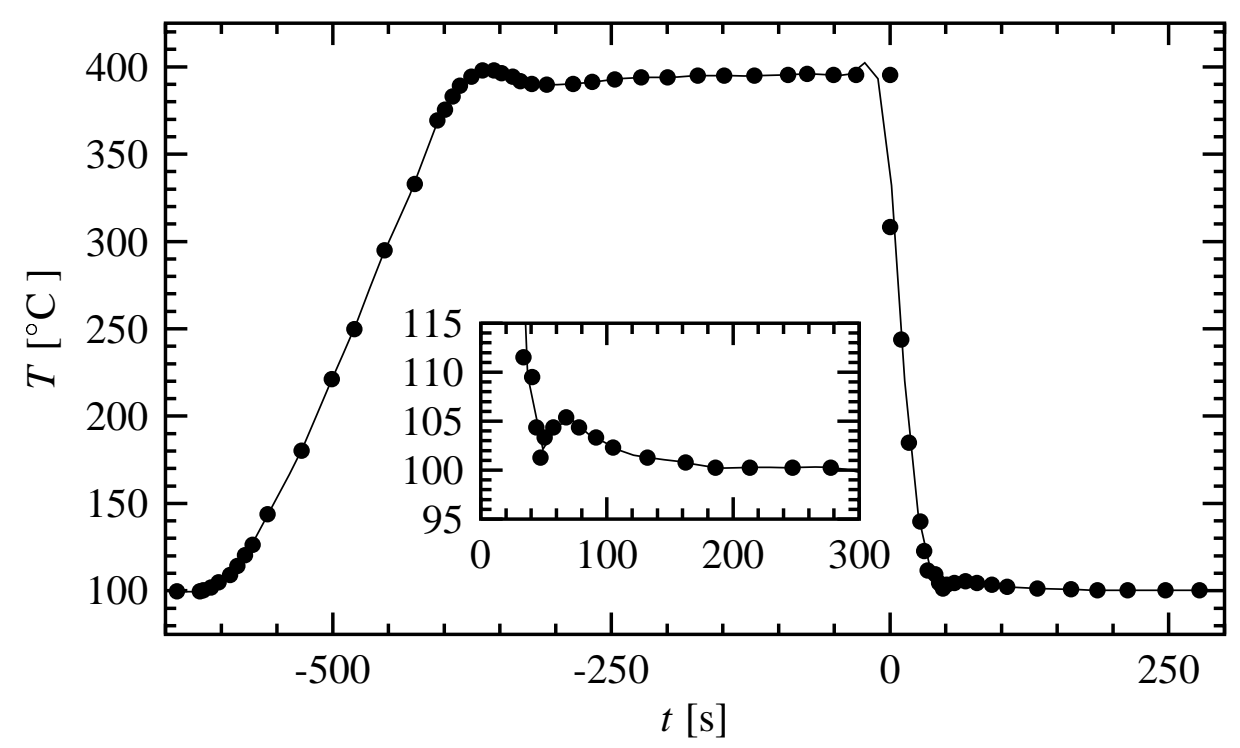

Abbildung .8: Die Abkühlkurve für das kinetische Experiment bei $100{ }^{\circ} \mathrm{C}$ mit $x_{0}=0.41$

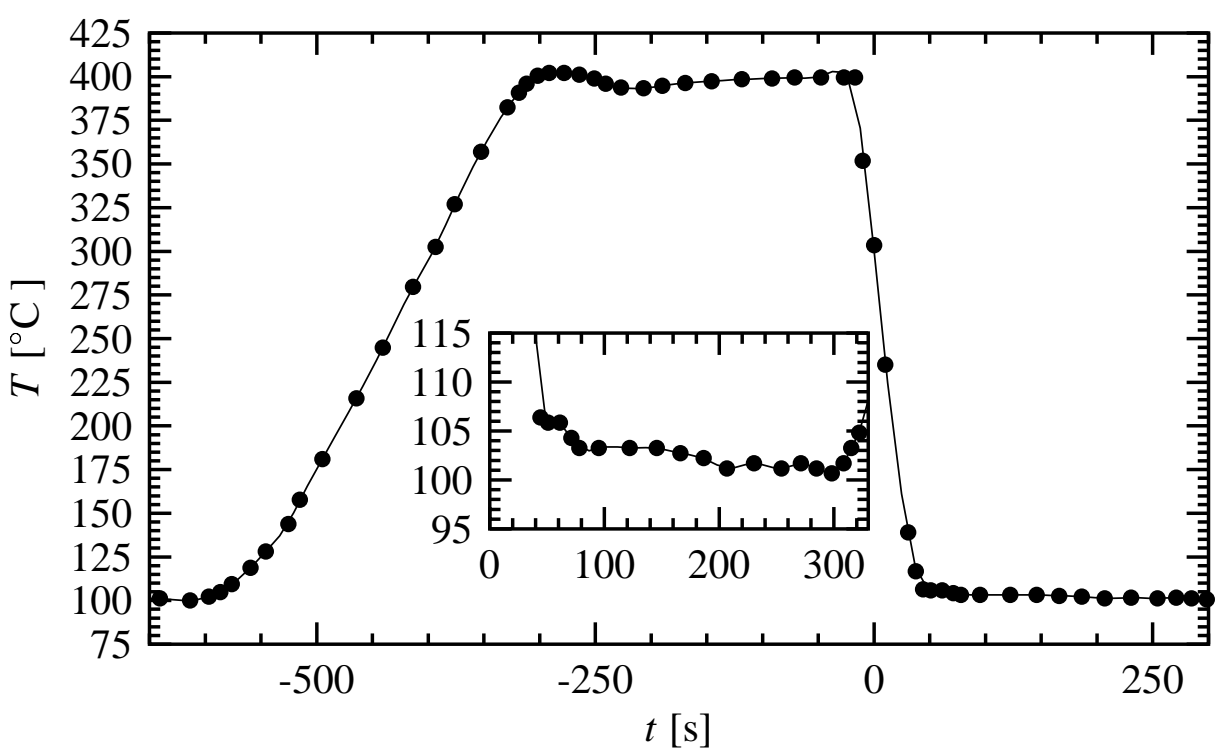

Abbildung .9: Die Abkühlkurve für das kinetische Experiment bei $100{ }^{\circ} \mathrm{C}$ mit $x_{0}=0.23$ 


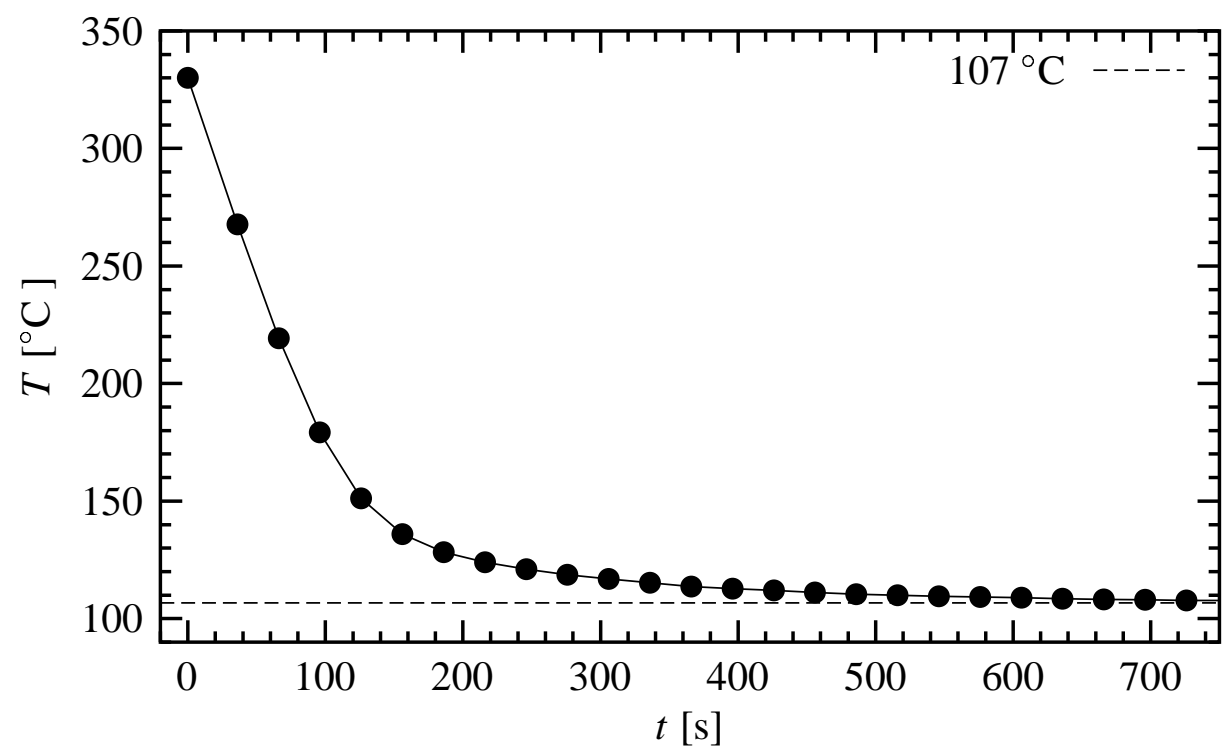

Abbildung .10: Die Abkühlkurve für das kinetische Experiment bei $107{ }^{\circ} \mathrm{C}$ mit $x_{0}=0.26$. Die Homogenisierungstemperatur betrug $330{ }^{\circ} \mathrm{C}$.

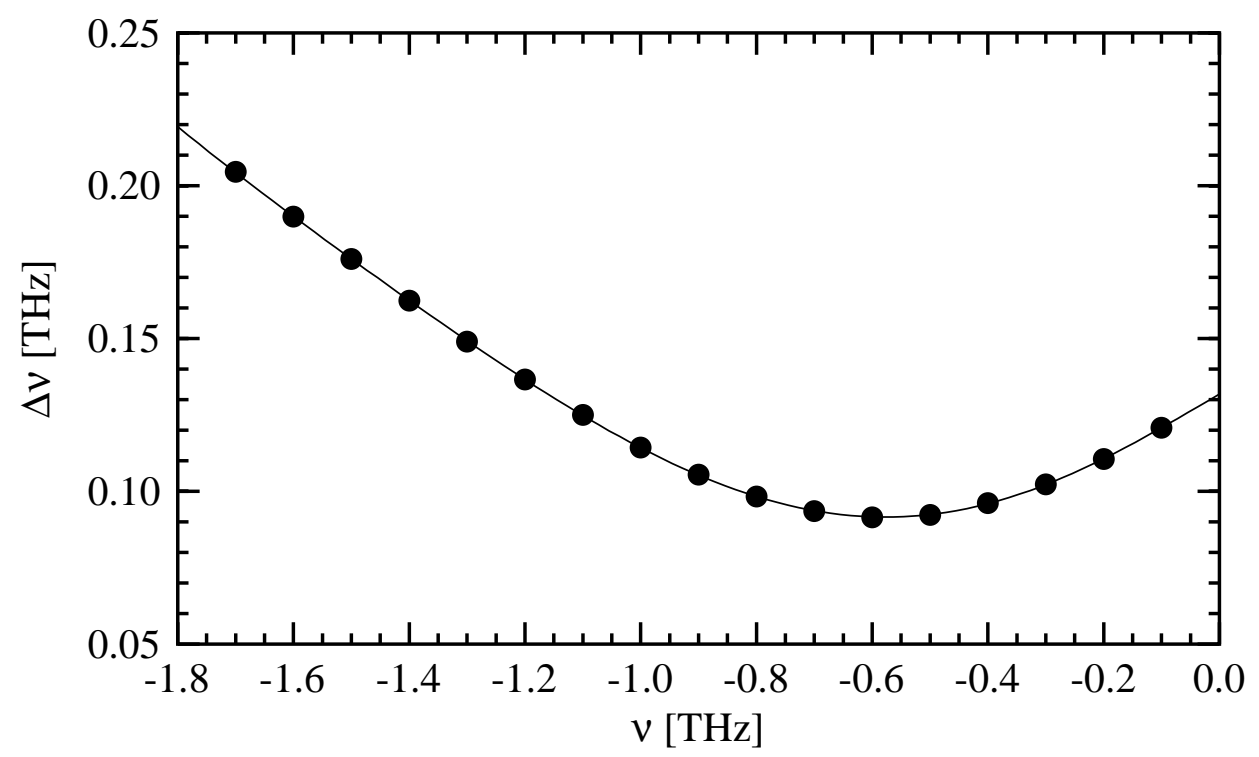

Abbildung .11: Die Auflösung des Spektrometers bei den kinetischen Experimenten für $x_{0}=$ 0.23 bei $100{ }^{\circ} \mathrm{C}$. Die Punkte sind die berechneten Halbwertsbreiten, die durchgezogene Line an die Werte angepaßtes Polynom. Die Auflösung unterscheidet sich von derjenigen für die $41 \%$ Probe, weil sie eine andere Mosaizität und Gitterkonstante hat. 


\section{Danksagung}

An erster Stelle sei Herrn Prof. Dr. G. Eckold für die Anregung zu dieser Arbeit und seine unentbehrliche Hilfs- und Diskussionsbereitschaft gedankt, ohne die diese Arbeit nicht möglich gewesen wäre.

Bei Dr. F. Güthoff möchte ich mich für die vielen kleinen Hilfen zwischen Tür und Angel, die Betreuung bei den Experimenten am UnIDAS, die Mitarbeit am IN-3, das herzhafte Lachen zum — wie ich fand - schlechtesten Zeitpunkt und die Gewißheit, daß bei Experimenten jedes Teil mindestens drei Mal benötigt wird, bedanken. Dr. W. Schmidt, gilt mein Dank für die verläßliche Rundum-Betreuung am IN-12 und das Vertrauen, daß die Steuerelektronik auch nach unseren Experimenten wieder wie gewohnt funktioniert. Dr. A. Hoser und H. Mitlacher danke ich für die Hilfe und Unterstützung bei den Experimenten am UNIDAS. Bei Dr. W. Schweika möchte ich mich für die unkomplizierte Bereitstellung von Meßzeit am DNS bedanken. Dr. B. Dorner und Dr. F. Demmel gilt mein Dank für die Betreuung während der Testmessungen am IN-3. Dr. H. Grimm seien die vielen Gespräche und Erläuterungen während der Aufenthalte am IFF in Jülich gedankt.

Stellvertretend für die mechanische Werkstatt möchte ich mich bei Herrn V. Meyer für all die Arbeiten an meiner Probenumgebung und insbesondere für den schnellen Probenofen bedanken. Genauso gilt mein Dank den elektronischen Werkstätten, für die Entstörung der stroboskopischen Experimente und die vielen kleinen Hilfen. Bedanken möchte ich mich auch bei den Mitarbeitern des IPC Göttingen, des ILL Grenoble und des FZ Jülichs, die diese Arbeit direkt oder indirekt ermöglicht oder zu ihr beigetragen haben.

Bei P. Elter möchte ich mich für die Zusammenarbeit und Hilfe bei den Experimenten in Jülich und Grenoble bedanken. Vielleicht experimentieren wir noch einmal gemeinsam in Jülich und stellen fest, daß die Bürgersteige nicht mehr um $20.00 \mathrm{~h}$ hochgeklappt werden, die Esso-Tankstelle ein vier Sterne Restaurant ist, der Irish Pub nicht mehr außerhalb liegt und daß zu Weihnachten ein Tannenbaum in der Reaktorhalle steht. F. Ratschow möchte ich meinen Dank für die vielen (off-topic) Diskussionen und Hilfen, den Gesprächen ,unter Chemikern“ im Büro und die Erfahrung, der letzte Gast in der Sonderbar zu sein, bedanken. Den Mitgliedern meiner Arbeitsgruppe Dr. H. Gibhardt, K. Elhisbiani, A. Gand, O. Wenzel und I. Kispert möchte ich für die Unterstützung in jeglicher Form meinen Dank aussprechen. Herrn Gollers möchte ich für seine Fürsprache danken. 
Ein großes „,Danke schön!“ gilt M. Czudnochowsky und insbesondere Dr. H. Gibhardt und A. N. Werner für das Korrekturlesen dieser Arbeit.

Vielen lieben Dank meinen Eltern, die mir mein Studium ermöglicht haben. Bei meinen Freunden — damit seid Ihr alle gemeint! — möchte ich mich für die Gewißheit eines Lebens nach der Chemie und vor dem Tod bedanken.

Liebe Anja: Danke! 


\section{Lebenslauf}

\section{Persönliche Daten}

Name Dirk Caspary

Geburtsdatum 18. Dezember 1971

Geburtsort Delmenhorst

\section{Schulbildung}

1978-79 Grundschule Varrel, Stuhr

1979-82 Grundschule Delmenhorst Deichhorst

1982-84 Orientierungsstufe am Schulzentrum West, Delmenhorst

1984-91 Gymnasium an der Willmsstraße, Delmenhorst

05/1991 Erlangung der allgemeinen Hochschulreife

\section{Studium und Berufstätigkeit}

1991-98 Studium der Chemie an der Universität Göttingen

06/1998 Abschuß als Diplom-Chemiker mit dem Thema der Diplomarbeit „Untersuchungen zur spinodalen Entmischung im System $\mathrm{AgCl}$ $\mathrm{NaCl}$ mittels Neutronenkleinwinkelstreuung “

10/1996 - 12/1998 wissenschaftliche Hilfskraft am Institut für Physikalische Chemie der Universität Göttingen

01/1999 - 05/2002 Wissenschaftlicher Mitarbeiter des Sonderforschungsbereiches „Festkörper fern ab vom Gleichgewicht"“

06/1999 - 10/2002 Wissenschaftlicher Mitarbeiter des Sonderforschungsbereiches „Komplexe Strukturen in kondensierter Materie von atomarer bis mesoskopischer Skala“

seit 10/2002 Wissenschaftlicher Mitarbeiter des Instituts für Physikalische Chemie der Universität Göttingen 